\title{
An Informed System Development Approach to Tropical Cyclone Track and Intensity Forecasting
}

\author{
by
}

Chandan Roy 
Cover image: Hurricane Isabel (2003), NASA, image in public domain.

Copyright (C) 2016 Chandan Roy

ISBN: 978-91-7685-854-7

ISSN 0345-7524

Printed by LiU Tryck, Linköping 2015

URL: http://urn.kb.se/resolve?urn=urn:nbn:se:liu:diva-123198 


\section{Abstract}

Introduction: Tropical Cyclones (TCs) inflict considerable damage to life and property every year. A major problem is that residents often hesitate to follow evacuation orders when the early warning messages are perceived as inaccurate or uninformative. The root problem is that providing accurate early forecasts can be difficult, especially in countries with less economic and technical means.

Aim: The aim of the thesis is to investigate how cyclone early warning systems can be technically improved. This means, first, identifying problems associated with the current cyclone early warning systems, and second, investigating if biologically based Artificial Neural Networks (ANNs) are feasible to solve some of the identified problems.

Method: First, for evaluating the efficiency of cyclone early warning systems, Bangladesh was selected as study area, where a questionnaire survey and an in-depth interview were administered. Second, a review of currently operational TC track forecasting techniques was conducted to gain a better understanding of various techniques' prediction performance, data requirements, and computational resource requirements. Third, a technique using biologically based ANNs was developed to produce TC track and intensity forecasts. Systematic testing was used to find optimal values for simulation parameters, such as feature-detector receptive field size, the mixture of unsupervised and supervised learning, and learning rate schedule. Five types of $2 \mathrm{D}$ data were used for training. The networks were tested on two types of novel data, to assess their generalization performance.

Results: A major problem that is identified in the thesis is that the meteorologists at the Bangladesh Meteorological Department are currently not capable of providing accurate TC forecasts. This is an important contributing factor to residents' reluctance to evacuate. To address this issue, an ANN-based TC track and intensity forecasting technique was developed that can produce early and accurate forecasts, uses freely available satellite images, and does not require extensive computational resources to run. Bidirectional connections, combined supervised and unsupervised learning, and a deep hierarchical structure assists the parallel extraction of useful features from five types of $2 \mathrm{D}$ data. The trained networks were tested on two types of novel data: First, tests were performed with novel data covering the end of the lifecycle of trained cyclones; for these test data, the forecasts produced by the networks were correct in $91-100 \%$ of the cases. Second, the networks were tested with data of a novel TC; in this case, the networks performed with between $30 \%$ and $45 \%$ accuracy (for intensity forecasts).

Conclusions: The ANN technique developed in this thesis could, with further extensions and up-scaling, using additional types of input images of a greater number of TCs, improve the efficiency of cyclone early warning systems in countries with less economic and technical means. The thesis work also creates opportunities for further research, where biologically based ANNs can be employed for general-purpose weather forecasting, as well as for forecasting other severe weather phenomena, such as thunderstorms. 


\section{Populärvetenskaplig sammanfattning}

Ett stort problem i cyklondrabbade områden är att människor ofta tvekar efter en första cyklonvarning mellan att utföra rekommenderat skyddsarbete hemmavid och försöka ta sig till ett skyddsrum, eller att fortsätta arbeta och stanna på plats och bevaka sitt hus mot inbrott. Problemet är att sedan, när cyklonen är nära, kan det vara för farligt att ge sig ut på vägarna. Ett delarbete i avhandlingen visar på att ett viktigt steg i att motivera människor att evakuera är att kunna erbjuda tillförlitliga tidiga cyklonvarningar.

Många länder som är sårbara för cykloner, såsom Bangladesh, saknar de ekonomiska resurser och den informationsinfrastruktur som krävs för att köra avancerade numeriska väderprognosmodeller, och har därför svårt att tillhandahålla tillförlitliga tidiga cyklonvarningar. I avhandlingen utvecklas en ny teknik för att förutse hur en cyklon kommer att röra sig och hur dess intensitet kommer att utvecklas.

Tekniken använder sig av biologiskt baserade artificiella neurala nätverk för att bearbeta data som ligger i ett rutmönster av geografiska punkter. Tekniken kan producera tidiga och tillförlitliga cyklonspår- och intensitetsprognoser, samtidigt som den använder fritt tillgängliga satellitdata och inte kräver superdatorer för att köras. En central egenskap hos tekniken är att den tar hänsyn till den rumsliga strukturen hos mätdata, som ger viktiga ledtrådar till spår och intensitetsförändringar hos cykloner.

Tekniken baseras på djupa neurala nät, där ett antal dubbelriktat kopplade lager av noder ligger i en hierarkisk struktur som är specifikt utformad för att hantera data med rumslig struktur. Tekniken kombinerar oövervakad och övervakad inlärning på varje beräkningsnivå. För att optimera prestanda har värden för centrala parametrar, såsom storleken på receptiva fält, och förhållandet mellan oövervakat och övervakat inlärning, systematiskt testats. Dessutom har en detaljerad analys gjorts av de interna representationer som utvecklas i dessa nät under träning, och dessa ger vägvisning om vilka faktorer i mätdata som är avgörande för en tillförlitlig cyklonprognos.

Den teknik och de resultat som presenteras i avhandlingen kommer att kunna förbättra effektiviteten i cyklonvarnings- och krishanteringssystem i länder som Bangladesh. Resultaten skapar också möjligheter för vidare forskning, där biologiskt baserade artificiella neurala nätverk kan användas för allmänna väderleksprognoser, samt för prognoser av svåra väderfenomen, såsom stormar och åskoväder. 


\section{Acknowledgements}

First and foremost I wish to thank my main advisor Rita Kovordányi. I would like to thank her for encouraging my research and for allowing me to grow intellectually. Her constructive guidance on both my research and thesis work, as well as on my career has been invaluable. She has been supportive since the days I started working on biologically based artificial neural networks as a master's student. She guided me academically and emotionally when writing this thesis and the research articles. Thanks to her, I had the opportunity to develop a tropical cyclone track- and intensity-forecasting technique using biologically based artificial neural networks.

My appreciation goes also to my co-advisors Mattias Villani and Henrik Eriksson. Their advices and comments on my thesis manuscript have been very helpful.

I want to thank Saroje Kumar Sarkar, Rajshahi University, Bangladesh, and Johan Åberg for helping me with questionnaire formulation and survey data analysis.

I am grateful to Raquib Ahmed, Rajshahi University, Bangladesh for his inspiring words and emotional support.

Thank you Jalal Maleki and Sture Hägglund for occasionally asking questions about my research and providing constructive advice.

Anne Moe, thank you for helping me with the administrative processes related to my thesis defense. I also want to thank all my fellow workers at the Department of Computer and Information Science (IDA) in general for the exceptional working environment that they have created. I always enjoyed my time at IDA.

I would like to thank Rajshahi University, Bangladesh, for granting me a paid leave to pursue $\mathrm{PhD}$ studies at the Department of Computer and Information Science, Linköping University, Sweden.

Finally, I want to thank each and every member of my family for their moral support during these years. Words cannot express how grateful I am to them for all of the sacrifices that they have made for my sake. To my beloved daughter Indira Roy, I would like to express my special thanks for being such a good girl and always cheering me up.

Chandan Roy

Linköping

December, 2015 


\section{Contents}

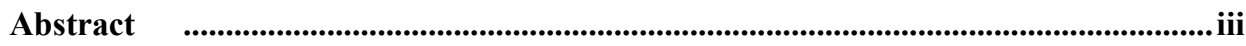

Populärvetenskaplig sammanfattning ..........................................................................

Acknowledgements ............................................................................................................... vii

Contents

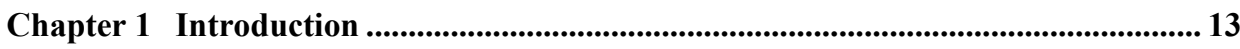

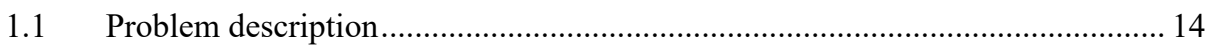

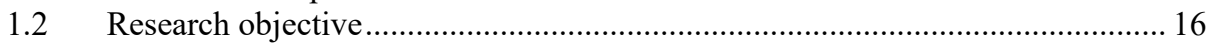

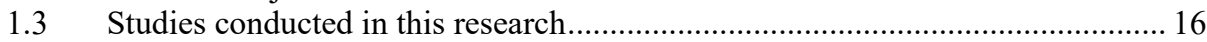

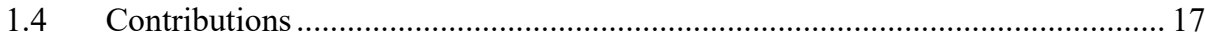

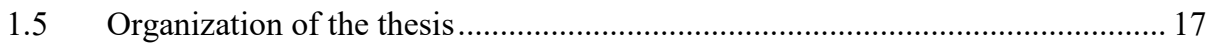

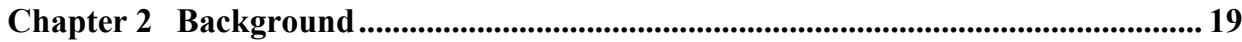

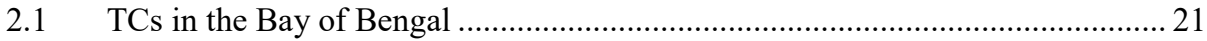

2.2 Factors governing TC track and intensity development.................................... 22

2.3 Technical processes involved in TC forecasting and warning ............................ 23

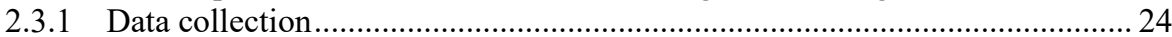

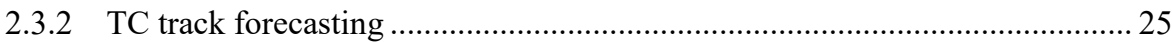

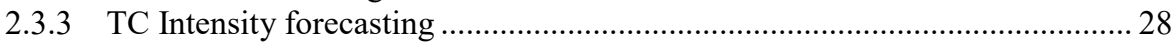

2.3.4 Warning message formulation and dissemination....................................... 29

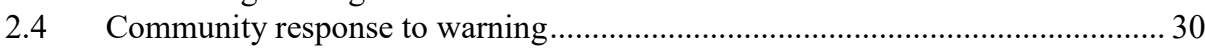

2.4.1 Incorporation of human perception into cyclone warning ........................... 31

2.5 Cyclone early warning system in Bangladesh................................................ 32

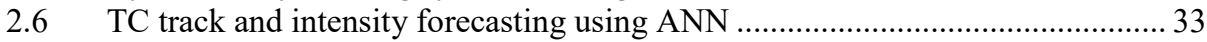

2.7 Biologically based ANN techniques for image processing ............................... 34

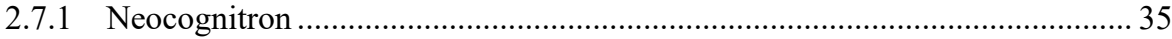

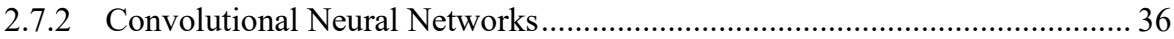

2.7.3 Saliency based visual attention models ......................................................... 37

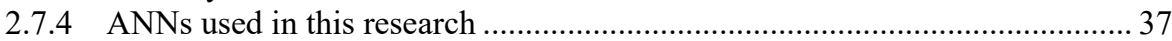

2.8 Exploratory study on TC movement direction prediction ................................... 38

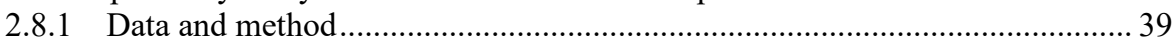

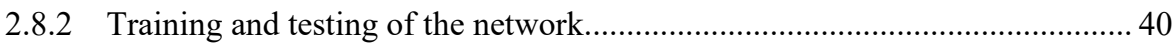

Chapter 3 Methodological considerations ................................................................... 43

3.1 Central and peripheral parts of this research ................................................. 45

3.2 Technological paradigm of this research.................................................. 47

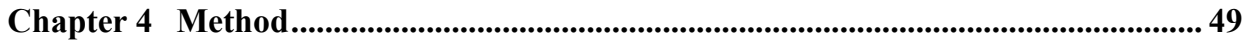

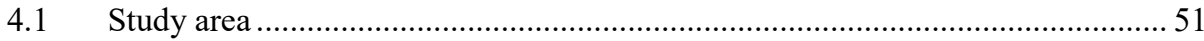

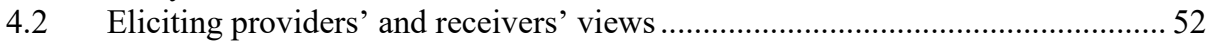




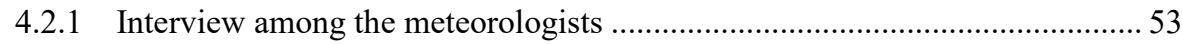

4.2.2 Questionnaire survey among the residents in the coastal areas....................... 55

4.3 TC track and intensity forecasting technique development ................................5 57

4.3.1 Efficiency of the simulation tool with respect to $2 \mathrm{D}$ image processing.......... 58

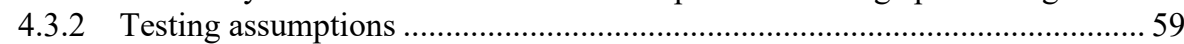

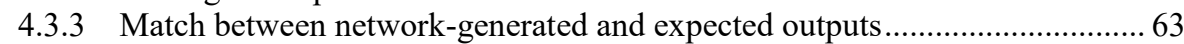

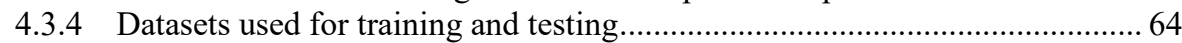

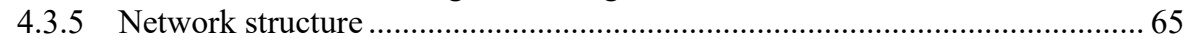

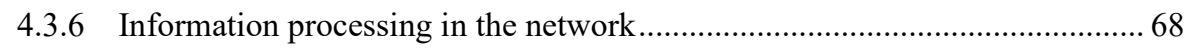

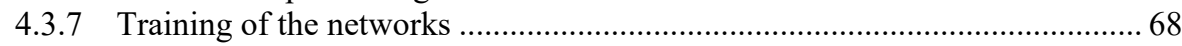

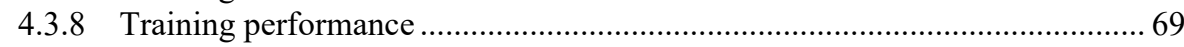

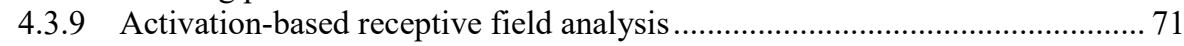

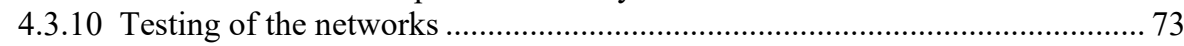

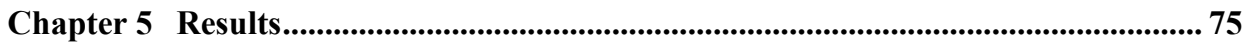

5.1 Results elicited from warning providers..................................................... 75

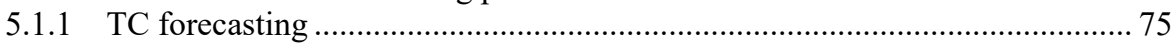

5.1.2 Warning message formulation and dissemination...................................... 76

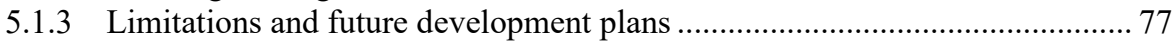

5.2 Results elicited from warning receivers ....................................................... 79

5.2.1 Warning message reception.................................................................. 79

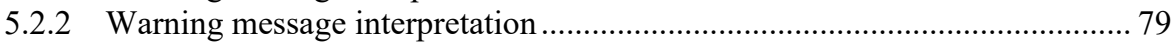

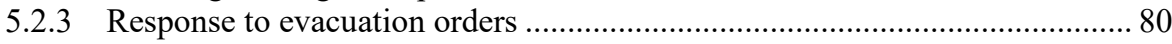

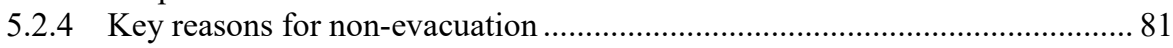

5.2.5 Satisfaction with the warnings and suggestion for improvement.................... 82

5.3 Results obtained during systematic parameter testing....................................... 83

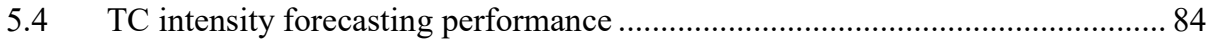

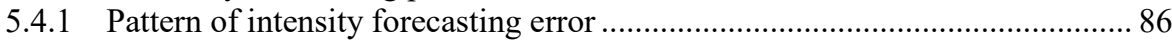

5.5 Combined TC track and intensity forecasting performance............................... 88

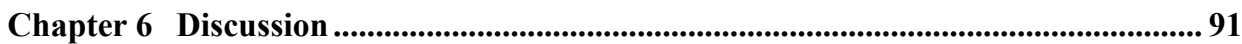

6.1 Problems associated with cyclone early warning in Bangladesh ...................... 91

6.2 TC track and intensity forecasting using biologically based ANNs.................. 91

6.2.1 TC Intensity forecasting ........................................................................ 92

6.2.2 Graphically-presented TC track and intensity forecasting — ongoing work.... 95

6.2.3 Prediction performance improvement ....................................................... 96

Chapter 7 Conclusions and future work .................................................................99

Chapter 8 References .................................................................................................. 101

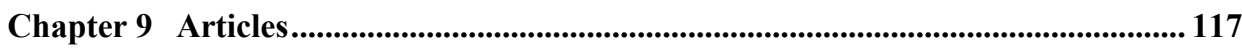

Article 1 The Current Cyclone Early Warning System in Bangladesh: Providers' and

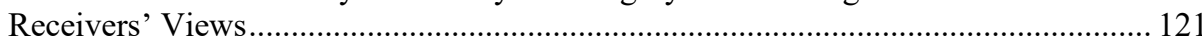

Article 2 Tropical Cyclone Track Forecasting Techniques-A Review ..................... 151

Article 3 Tropical Cyclone Track Forecasting ......................................................... 207

Article 4 Local feature extraction -what receptive field size should be used? ............ 243

Article 5 Bidirectional hierarchical neural networks-Hebbian learning improves

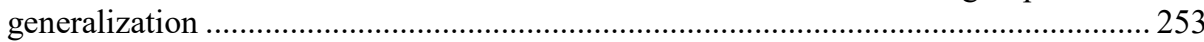

Article 6 A biologically based machine learning approach to tropical cyclone intensity

forecasting 
Appendix A Cyclone track forecasting using biologically based ANNs................... 289

Appendix B TC signaling system for the maritime ports.......................................309

Appendix C TC signaling system for the river ports ...............................................312

Appendix D Questions used for the in-depth interview.......................................... 314

Appendix E Questionnaire used for the survey .....................................................3 318

Appendix F Per-recorded warning messages........................................................... 323

Appendix G Generalized TC signaling system ........................................................ 327 


\section{Chapter 1}

\section{Introduction}

Since the dawn of our existence, different types of disasters have adversely affected us. In response, individuals and societies have taken measures both to reduce their exposure to such disasters and to mitigate their consequences. These measures include the development of techniques for disaster risk assessment and for forecasting disasters before actual impact. Actions are usually also taken to assess the incurred losses, and to perform post disaster response and recovery activities. All these efforts have the same overarching goal: disaster management. As disasters often exhibit a recurring pattern, it is possible to forecast them and reduce their effects, even though the disasters cannot be prevented from happening (Haddow, Bullock, \& Coppola, 2008).

Among all natural disasters, hydro-meteorological disasters have been the most frequent, have affected the greatest number of people, and have caused the highest amount of economic losses globally during the last century (CRED, 2013). Tropical Cyclones ${ }^{1}$ (TCs) are the most common and the most destructive among all hydro-meteorological disasters (CRED, 2013; Sahni \& Ariyabandu, 2004). TCs are characterized by that they (Chan \& Kepert, 2010):

1. Form over tropical waters, but hit coastal regions

2. Usually occur during the periods of seasonal transitions

3. Are governed by a complex interaction between thermo-dynamic and hydrodynamic processes, and

4. Can cause severe destruction in a large area through strong up-shore wind and flooding.

These unique characteristics have made accurate TC track and intensity forecasting an important, but complex matter. Considerable efforts have been made to reduce TC track and intensity forecasting error during the past decades, with the aim to deliver accurate and informative warnings to the disaster management authorities and to residents living in coastal areas (Cooper \& Falvey, 2010). However, different level of success in TC forecasting (NCAR, 2014), and variations in TC-induced losses between countries (CRED, 2013), reflect that the cyclone early warning systems-regarded as a social-technical system - practiced in different countries are not always efficient.

A cyclone early warning system consists of two main processes (Parker, 1999; Takeuchi, 2008). First, the technical process, where the data used for TC forecasting are collected,

1 Also called hurricanes or typhoons. 
TCs are forecasted on the basis of the collected data, and warning messages are formulated and disseminated on the basis of the produced forecasts. Second, the residents' response process, where warning messages are received, interpreted, and acted on by the residents living in cyclone-prone coastal areas. In this respect, the technical process and the social processes are interconnected and are equally important for improved cyclone emergency management (Drabek, 1999; Parker, 1999; Quarantelli, 1990; WMO, 1989).

Considering its importance in cyclone disaster management, researchers have placed more and more emphasis on identifying issues that can influence the effectiveness of cyclone early warning systems (I. Davis, Sanderson, Parker, Stack, \& Lee, 1998; Drabek \& Hoetmer, 1991; C. E. Haque, 1997; C. E. Haque \& Blair, 1992). These research efforts have contributed to the identification of factors that seem to influence residents' evacuation behavior in cyclone-prone areas. These include:

Factors associated with the warning message itself. For example, the disseminated warning messages could be (perceived as) unreliable, the warning message could be incomplete, with no clear guideline for evacuation given (Dash \& Gladwin, 2007; Gladwin, Lazo, Morrow, Peacock, \& Willoughby, 2007; U. Haque et al., 2012; B. K. Paul \& Dutt, 2010).

The cyclone risk level of an area. Whether the residents are aware of the risk of living in a particular area (Baker, 1991; FEMA (Federal Emergency Management Agency), 2010; Sahni \& Ariyabandu, 2004).

Factors associated with infrastructure. For example, the location and the condition of houses, the availability and condition of cyclone shelters, and the availability of road and other transportation facility (Asgary \& Halim, 2011; C. E. Haque \& Blair, 1992).

Underlying causes of vulnerability to TCs, such as lack of access to resources (or lack of capacity to use resources to secure livelihood), lack of education and training, and fragmentation in the community (Alam \& Collins, 2010; Asgary \& Halim, 2011; Letson, Mileti, \& Lazo, 2007; Phillips \& Morrow, 2007).

Though various studies have identified the technical and the residents' response processes to be important in determining the effectiveness of a cyclone early warning system (Brady, 2005; Letson et al., 2007; Phillips \& Morrow, 2007), the two processes have not been considered together in the same study when evaluating early warning systems.

\subsection{Problem description}

The current cyclone early warning system in Bangladesh seems ineffective, as it often fails to elicit the expected response to evacuation orders from the residents living in the affected coastal areas (Mallick, Witte, Sarkar, Mahboob, \& Vogt, 2009; B. K. Paul, 2012; B. K. Paul $\&$ Dutt, 2010). Though the contributions of inaccurate and uninformative warnings to nonevacuation have already been identified in previous research (U. Haque et al., 2012; B. K. Paul, 2012; B. K. Paul \& Dutt, 2010; B. K. Paul, Rashid, Islam, \& Hunt, 2010), the reasons for the dissemination of inaccurate and uninformative warnings by the Bangladesh Meteorological Department (BMD) have remained unidentified.

It is important for residents that the warnings they receive are reliable in order for them to take necessary action well before a TC hits the coast. As the residents do not perceive the warnings as trustworthy (Chowdhury, 2002; Miyan, 2006; B. K. Paul \& Dutt, 2010), very 
often they seem unwilling to make an evacuation at an early stage. The residents also consider saving their property and avoiding unnecessary preparatory work more important than making an evacuation at an early, unreliable stage. As the TC approaches the coast gradually, it becomes increasingly easier for meteorologists to provide accurate information about the TC's intensity and location of landfall in the warning messages (SWC (Storm warning Center), 2007, 2013). However, at this later stage, as the TC is about to cross the coastline, there is often not sufficient time to make an evacuation. This situation particularly highlights the need for reliable early forecasts.

TC forecasting centers around the world deploy multiple forecasting techniques, ranging from climatology and persistence to dynamical-numerical modeling, to produce forecasts for TC track and intensity (DeMaria, 2009; NCAR, 2014; NOAA, 2012). Advancements in numerical atmospheric modeling at global and regional scales and progress in satellite technologies have contributed to a gradual improvement in TC motion forecasting accuracy during the past two decades (Evans \& Falvey, 2012; Franklin, 2010; Rogers et al., 2006). However, TC intensity forecasting accuracy has not been much improved during the same time period, mainly due to:

1. Poor understanding of the physical processes governing TC intensity (Rogers et al., 2006)

2. Deficiency of observational data from the TC vortex ${ }^{2}$ (Bender \& Ginis, 2000; DeMaria, Knaff, \& Sampson, 2007), and

3. Insufficient model resolution (Bender \& Ginis, 2000; Demaria \& Kaplan, 1994; Emanuel, DesAutels, Holloway, \& Korty, 2004).

This disparate prediction performance for TC track and intensity implies that TC intensity prediction is difficult compared to track prediction. Even with multiple forecasting techniques in operation, TC forecasting centers are still struggling to handle this difficulty. Considering the operational forecasting techniques' limitations in TC intensity forecasting (Aberson et al., 2010; DeMaria et al., 2007; Rogers et al., 2006), as well as the inefficiencies of the current cyclone early warning system in Bangladesh (Akhand, 2003; U. Haque et al., 2012), new techniques, equally effective in TC track and intensity forecasting need to be explored.

This research employs an informed system development approach to identify problems of the current cyclone early warning system in Bangladesh and tries to solve some of the identified problems by considering the possibility of using biologically based ANNs for producing accurate TC track and intensity forecasts. The developed TC track and intensity forecasting technique is also expected to satisfy a number of criteria, which could render the technique a future effective, low-cost alternative to the currently operational forecasting technique. The new technique should: (a) use freely available satellite-recorded data as inputs (predictors), (b) not require extensive computational resources to run, (c) be capable of producing high-accuracy long-term forecasts in the future, and (d) be easily deployed and produce forecasts quickly.

2 A TC vortex is an area of rotating wind surrounding a low-pressure center, which usually extends high up in the atmosphere. The rotational direction is counterclockwise in the northern hemisphere and clockwise in the southern hemisphere. 


\subsection{Research objective}

The objective of the work presented in this thesis is to contribute to improved cyclone early warning systems in countries with less economic and technical means. The questions (research problems) that this thesis addresses to meet the objective are:

1. Understanding the problems associated with the current cyclone early warning system in Bangladesh.

2. Address technical challenges revealed in the previous question, and investigate if biologically based ANNs are feasible for producing accurate TC track and intensity forecasts.

\subsection{Studies conducted in this research}

In order to address the above research questions, five interrelated studies were conducted and published as research articles:

1. First study: Evaluation of the efficiency of a cyclone early warning system. The primary goal of this study was to elicit the views both of the meteorologists at the Bangladesh Meteorological Department (BMD) and of the residents in the coastal areas of Bangladesh on the current cyclone early warning system in Bangladesh. Research article published on the basis of the findings of this study:

- Roy, C., Sarkar, S. K., Åberg, J., \& Kovordanyi, R. (2015). The current cyclone early warning system in Bangladesh: Providers' and receivers' views.

International Journal of Disaster Risk Reduction, 12, 285-299. http://doi.org/10.1016/j.ijdrr.2015.02.004

2. Second study: Review of tropical cyclone track forecasting techniques. The aim of this study has been to get a detailed overview of the TC track forecasting techniques that are/were in use at various TC forecasting centers around the world. Outcomes of the second study were published as research articles:

- Roy, C., \& Kovordányi, R. (2012). Tropical cyclone track forecasting techniques-A review. Atmospheric Research, 104-105, 40-69.

http://doi.org/10.1016/j.atmosres.2011.09.012

- Roy, C., \& Kovordányi, R. (2015). Tropical Cyclone Track Forecasting. In Encyclopedia of Natural Hazards. Taylor \& Francis Group.

3. Third study: Systematic evaluation of the influence of receptive field size on local feature extraction. The focus of this study has been to explain the role of Receptive Field (RF) size in the extraction and recognition of meaningful features from the 2D images. Research article published on the basis of the outcomes of this study:

- Kovordányi, R., Roy, C., \& Saifullah, M. (2009). Local Feature ExtractionWhat Receptive Field Size Should Be Used? In Proceedings of International Conference on Image Processing, Computer Vision and Pattern Recognition. 13-16 July, Las Vegas, NV.

4. Fourth study: Systematic evaluation of the effect of Hebbian learning on generalization. The main purpose of this study was to understand, how Hebbian (unsupervised) learning can be combined with supervised learning to enhance 
generalization performance of a biologically based ANN. Findings of this study were published as research article:

- Saifullah, M., Kovordanyi, R., \& Roy, C. (2010). Bidirectional Hierarchical Neural Networks: Hebbian Learning Improves Generalization. In Proceedings of the Fifth International Conference on Computer Vision Theory and Applications (Vol. 1, pp. 105-111). 17-21 May, Angers, France.

5. Fifth study: TC intensity as well as combined track and intensity forecasting using biologically based ANNs. The main objective of this study has been to develop a TC track and intensity forecasting technique using biologically based hierarchical ANNs, where multi-instrument infrared, sea-level pressure, ocean heat content, wind direction, and wind speed images are used for training and testing. Results produced by the TC intensity-forecasting network are provided in research article:

- Roy, C. and Kovordanyi, R. (manuscript) A biologically based machine learning approach to tropical cyclone intensity forecasting.

\subsection{Contributions}

The contributions of the research can be summarized under the following two headings:

1. Scientific contributions:

a) Identifies the reasons for the dissemination of inaccurate and uninformative warnings by BMD.

b) Offers an efficient ANN technique for producing good accuracy TC track and intensity forecasts.

c) Creates opportunities for further research, where biologically based machine learning can be employed for general-purpose weather forecasting as well as for forecasting other severe weather phenomenon, such as thunderstorms.

2. Other contributions:

a) Provides a detailed overview of the current cyclone early warning system in Bangladesh, which can guide emergency management authorities to formulate better plans for managing cyclone emergencies. The findings of this research are expected to be relevant for improving cyclone early warning systems in other cyclone-prone countries, where socio-economic conditions and technical settings at the weather forecasting centers are similar to Bangladesh.

b) Analysis of activation patterns in the network could elicit, which factors are more important for an accurate forecast of TC track and intensity development.

\subsection{Organization of the thesis}

The chapters of this thesis are organized in the following way:

Chapter 1 describes the thematic context of the study, the problems to be addressed, the research objectives, and the contributions of the research.

Chapter 2 is devoted to reviewing the literature, which is related to this research. This chapter describes the processes involved in TC forecasting and warning, and looks at the 
cyclone early warning system in Bangladesh. The TC track and intensity forecasting technique developed in this thesis use multiple-satellite-recorded images as predictors and biologically based ANNs as forecasting tool. Therefore, the reasons for using ANNs for TC track and intensity forecasting, and a description of biologically based ANNs are also provided in this chapter.

Chapter 3 describes the methodological issues of this research. The scientific methodology followed in this research, the technological paradigm of this research are the key issues described in this chapter.

Chapter 4 describes the method used for conducting the studies in this research. The process of data collection from the providers (meteorologists at BMD) and the receivers (residents in the coastal areas of Bangladesh) of warning message, the approach followed to construct valid networks for TC forecasting, the data used for TC track and intensity forecasting, the architecture of the networks used for forecasting, information processing in the network, training and testing procedures, and a method for describing developed activation patterns in ANN are provided in this chapter.

Chapter 5 presents the results of the surveys among the providers and receivers of early warning, as well as the TC track and intensity forecasting performance produced by the networks.

The results are discussed in chapter 6 . This chapter also contains a detailed description of how TC track and intensity forecasting performance can be improved and how combined track and intensity forecasts can be produced using the developed technique.

Chapter 7 consists of the conclusions and a brief description of future work.

Chapter 8 presents the list of references cited in this thesis.

The six articles accompanying this thesis are provided in chapter 9. 


\section{Chapter 2}

\section{Background}

Hydro-meteorological disasters are unique among all natural disasters with their huge destruction capacity and frequent occurrence (Figure 2-1). These characteristics also bring forth the necessity of developing effective management measures so that inflicted loss can be minimized. For effective management of hydro-meteorological disasters, issues that should be given extra care include (Sahni \& Ariyabandu, 2004; Takeuchi, 2008):

1. The rapid growth of human population, the ever-increasing human activities in disaster prone areas, poverty, and poor governance are among the major causes of growing vulnerability to hydro-meteorological disasters. Warming of the global environment is working here more as a strong accelerator and facilitator than a direct or indirect cause.

2. Mitigation and adaptation to hydro-meteorological disasters should be accomplished under a common governing principle. Drastic improvement in mitigation and adaptation is only possible through combined technological innovation and human adjustment to disasters.

3. Advanced technologies for observing, analyzing, and forecasting natural hazards play an increasingly important role in disaster management. However, it is important to keep in mind that science can reduce the effects of disaster only if it is properly incorporated into overarching societal countermeasures, taking into account human activities and basic vulnerability.

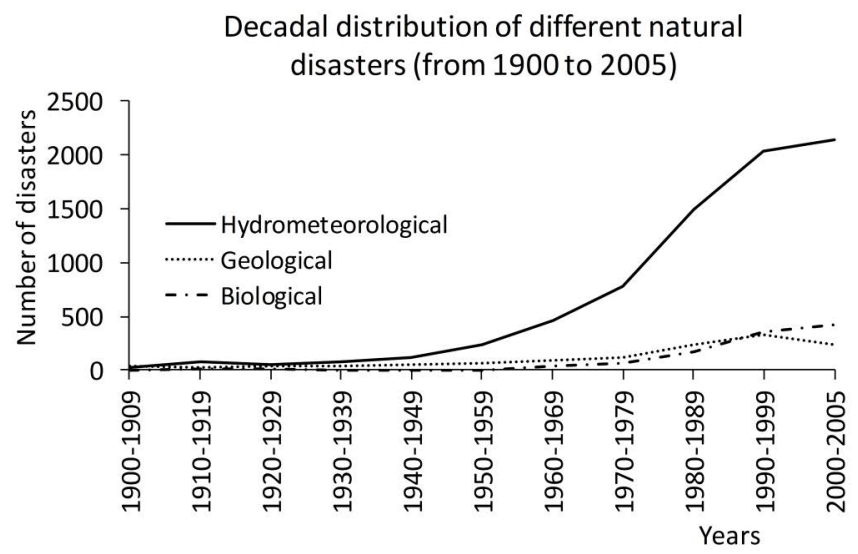

Figure 2-1. Frequency of natural disasters by origin during the last century. Created based on the natural disaster frequency database prepared by CRED (2013). 
Impact mitigation, adaptation, societal vulnerability reduction, and precise forecasting are the key to reduce losses created by hydro-meteorological disasters (Sahni \& Ariyabandu, 2004). Basically, these are the activities that are performed in the four-phase disaster management approach, which was first proposed by Whittaker (Whittaker, 1979). This four-phase management approach (mitigation $\rightarrow$ preparedness $\rightarrow$ response $\rightarrow$ recovery) is generic to all disasters, also for TCs. As place, time of occurrence, extent, governing factors, and destructive power can vary between disasters, activities performed under the generic four-phase approach needs to be adjusted to address various issues associated with a particular disaster and to handle the created emergencies in a better way (Coppola, 2006; Sahni \& Ariyabandu, 2004).

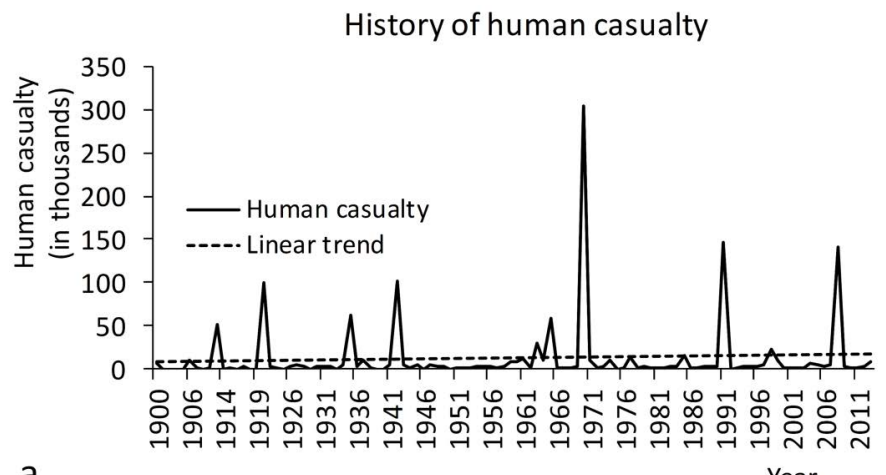

a.

Year

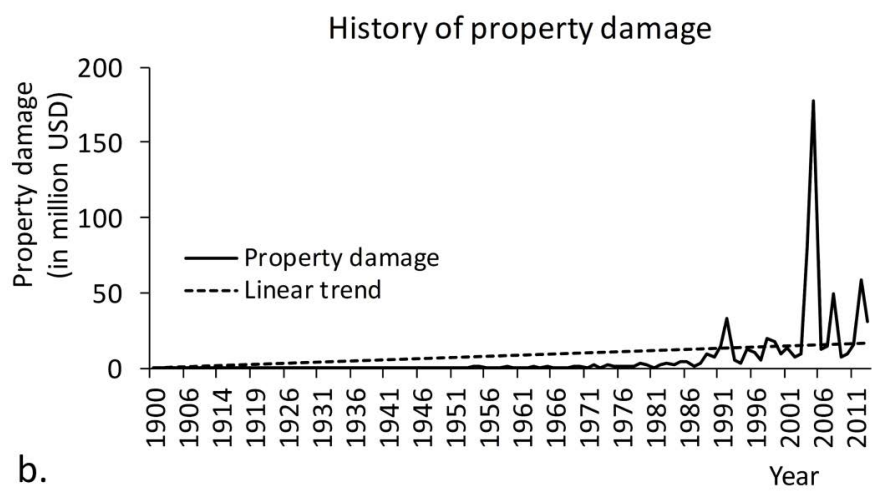

Figure 2-2. a. Illustrates human casualty and b. illustrates property damage caused by TCs during the period 1900 to 2011. Continuous line illustrates the observed statistics and dotted line illustrates the trends. Created based on TC damage database prepared by CRED (2013).

For TCs, though the activities performed under each of the four phases are important, effective early warning plays a crucial role in the whole TC emergency management process (Sahni \& Ariyabandu, 2004; Zschau \& Küppers, 2003). Which part of the population should be warned and which areas should be evacuated can best be determined on the basis of accurate forecasting of TCs. The damages caused by TCs can be effectively reduced if accurate and informative warnings can be delivered in time to the emergency management authorities and to the residents in the probable affected areas (Islam, Ullah, \& Paul, 2004; B. K. Paul, 2009, 2012; B. K. Paul \& Dutt, 2010; Sahni \& Ariyabandu, 2004). 
As residents' reaction to a warning message is largely governed by their trust in the disseminated warning, the information contained in the warning message needs to be precise (Anderson-Berry, 2003; Thomalla \& Schmuck, 2004). Imprecise forecasting of TC track and intensity seems to be one of the key reasons that property damage and human casualties have remained beyond control despite of adopting various protective and preparedness measures by countries affected by TCs (ADRC (Asian Disaster Reduction Center), 2005; Sahni \& Dhameja, 2004; Zschau \& Küppers, 2003). According to the statistics prepared by the Centre for Research on the Epidemiology of Disasters (CRED), property damages as well as human casualties created by the TCs could not been reduced during the last century (Figure 2-2); instead they have increased gradually during that period of time.

\subsection{TCs in the Bay of Bengal}

The Bay of Bengal (BoB) is an ideal breeding ground for TCs. Countries around the BoB, such as Bangladesh, India, and Myanmar are mainly affected by TCs that form over the southern part of the BoB. Formation of TCs over the BoB is often associated with seasonal transitions. The south-Asian monsoon advances or retreats with northward or southward shifts of the inter-tropical convergence zone. During the periods of seasonal transitions (mid-March to May and mid-September to mid-December) high instability in the atmosphere near the equatorial region of the BoB contributes to the development of lowpressure zones that later intensify into TCs upon the availability of favorable conditions (Debsarma, 2001).

\section{Monthly distribution of TCS \\ in the Bay of Bengal}

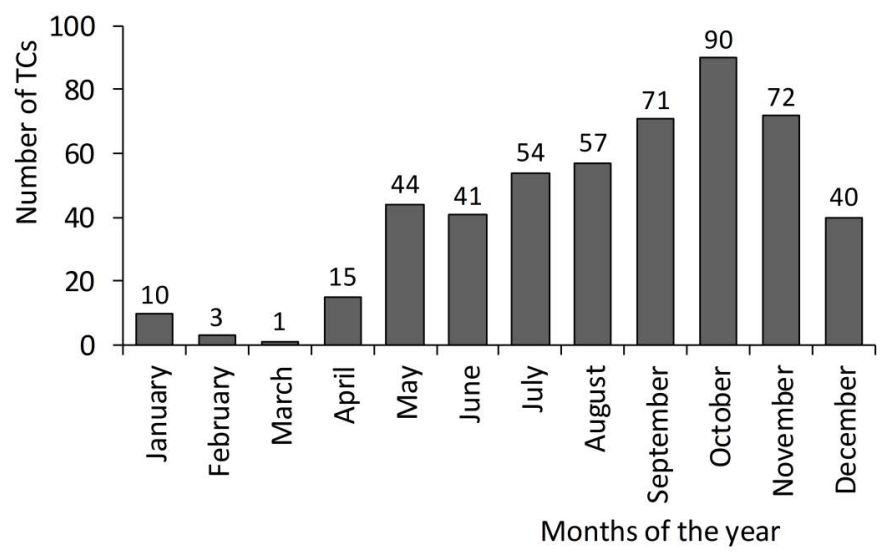

Figure 2-3. Monthly distribution of TC formation over the Bay of Bengal during the period 1940 to 2009. Created based on TC records archived by the Bangladesh Meteorological Department (BMD, 2010).

The initial formation of low-pressure zones and the development of these low-pressure zones into TCs are affected by several factors. Among these factors, the more influential ones include (Chan \& Kepert, 2010; Gray, 1968): (a) heat accumulated in the ocean waters, (b) sea surface temperature, (c) moisture content of the mid-tropospheric level, (d) vertical 
wind shear, (e) pre-existing atmospheric disturbances, and (f) the Coriolis force. The absence of some of these meteorological and oceanic factors render January, February, and March the least TC-prone months (BMD (Bangladesh Meteorological Department), 2010) in the $\mathrm{BoB}$ (Figure 2-3). TC formation frequency starts to increase after March and it reaches its peak in the month of October (Figure 2-3).

\subsection{Factors governing TC track and intensity development}

During the past few decades, considerable research effort has been devoted to understanding the processes responsible for TC motion and intensity change (Chan \& Kepert, 2010; Sampson, Jeffries, \& Neumann, 1995; Sampson, Jeffries, Neumann, \& Chu, 1995). While the factors governing TC motion work at synoptic (large) scale, and are relatively obvious and not overly complex (Bin, Elsberry, Yuqing, \& Liguang, 1999; Sampson, Jeffries, \& Neumann, 1995), the factors responsible for TC intensity change operate on multiple scales and are often difficult to observe. As a result, exactly which physical processes govern TC intensity and how they contribute to TC intensity change are not well-understood still today (Aberson et al., 2010; Rogers et al., 2006).

The factors governing TC motion include:

1. Large-scale environmental circulation is the most important factors that determines TC motion (Chan \& Kepert, 2010; Neumann, 1992).

2. In situation when the influence of environmental circulation on a TC is small, the beta effect (the variation of the Coriolis force with latitude) plays the main role in the determination of TC track (Chan, 1982; Holland, 1983).

3. Binary vortex interaction, or the Fujiwhara effect, is observed when more than one TC is formed in the same basin, close to each other (around $1000 \mathrm{~km}$ away from each other) (Fujiwhara, 1923). In such a situation, the two TCs tend to begin mutual orbiting in a counterclockwise direction (in the Northern hemisphere) around a point between the two TC vortices. This point is determined by the relative mass and intensity of the two TCs. When two TCs are following this complicated track, the track of the smaller TC is more influenced compared to the bigger $\mathrm{TC}$ and sometimes these two vortices may also merge to form a bigger vortex (Chan \& Lam, 1989; Holland \& Lander, 1993; Lander, 1995).

4. Processes like vertical wind shear and diabetic heating differ considerably at various pressure levels and have considerable influence on TC motion (Ngan \& Chan, 1995). As vertical structure of a TC vortex is not uniform, influences of beta effect, advection, and vertical wind shear are different (Flatau, Schubert, \& Stevens, 1994; Shapiro, 1992; Wang \& Li, 1992; C.-C. Wu \& Emanuel, 1993).

5. Experimental results show that inclusion of the Coriolis force in the x-momentum equation due to vertical motion causes a south-westward displacement of a TC with a speed of $\sim 1 \mathrm{~km} / \mathrm{h}$ even on a $f$ plane (Chan \& Kepert, 2010). This effect on TC motion is commonly known as gamma effect.

6. Before landfall, as winds within a TC interact with land, moisture supply from the warm ocean waters is also reduced. This situation might have considerable influence on TC track as well as on TC intensity (Chan \& Kepert, 2010; Kuo, Williams, Chen, \& Chen, 2001). 
Factors that are currently known to have influences on TC intensity change include:

1. Oceanic heat is the main source of energy that propels the whole low-pressure system over water (Cione \& Uhlhorn, 2003; G. Goni et al., 2009; G. J. Goni \& Trinanes, 2003; Shay, Goni, \& Black, 2000). This heat is transferred in the atmosphere in the form of moisture and converted into kinetic energy.

2. Weak vertical wind shear is favorable for TC intensification. As strong vertical wind shear takes the heat (transferred from the ocean) out of the TC vortex, it inhibits TC intensification (DeMaria, 1996; Wong \& Chan, 2004).

3. Inter-tropical convergence zone stimulates TC intensification through accelerating convective heat transfer.

4. When a TC moves over land, the underlying energy source is cutoff and it decays gradually (Merrill, 1987). A TC decays faster when it passes over mountainous regions than it does over flat land. Likewise, a TC may re-intensify again when it reenters into warm oceans (Brand \& Blelloch, 1973; Shoemaker, 1991).

5. An intense TC with high internal instability has the potential to become stronger unless it moves over land, moves over cool water, dry air gets in and/or strong vertical shear removes the heat from the TC column.

6. The eddy flux convergence of relative angular momentum at 200 mbar level has significant impact on TC intensification (DeMaria, Kaplan, \& Baik, 1993).

Recent observations in various TC formation basins have identified several additional factors that can also cause significant change in TC intensity:

1. Ocean eddies cause rapid intensification in TCs through enhancing heat transfer from the ocean (Powell, Vickery, \& Reinhold, 2003; C.-C. Wu, Lee, \& Lin, 2007).

2. In the western North Pacific basin, TCs are more intense and have longer lifespan in the El Niño-Southern Oscillation (ENSO) years compared to the La Niña years (Camargo \& Sobel, 2005).

3. TC rain bands have interactions with its eye-walls and thus can influence the intensity of a fully grown TC (Houze et al., 2006).

4. TC transition speed (Zeng, Chen, \& Wang, 2008) and the Saharan air (Dunion \& Velden, 2004; Shu \& Wu, 2009) also have significant influence in TC intensity change in the Atlantic basin.

\subsection{Technical processes involved in TC forecasting and warning}

Three consecutive processes are involved in TC forecasting and warning: (a) data collection, (b) TC forecasting, and (c) warning message dissemination (Figure 2-4). These processes can be further divided into sub-processes, on the basis of data collection methods, geographical characteristics of the affected region, and warning message dissemination plans (Zschau \& Küppers, 2003).

The process of TC forecasting starts with manual or automated detection of low-pressure systems on the basis of data obtained from multiple sources (Ho \& Talukder, 2008; Zou, Lin, Xie, Lang, \& Cui, 2010). After detection of a low-pressure system over the sea, a detailed monitoring process is initiated so that the meteorologists can have access to 
maximum possible information about the low-pressure system that could be used to analyze its development. A low-pressure system can develop into a TC, and would then finally decay in four successive stages (Chan \& Kepert, 2010; Debsarma, 1999, 2001): (a) formation stage, (b) development stage, (c) mature stage, and (d) decay stage. These four stages together constitute the lifecycle of a TC. TC lifecycles are usually 3-5 days long but sometimes, depending on the atmospheric and oceanic conditions, TCs may have a life cycle of up to 2-3 weeks (Debsarma, 1999). As both track and intensity can change at any stage of a TC's lifecycle, meteorologists continue with monitoring and forecasting relevant processes until it decays over water or crosses the coast, and eventually decays over land.

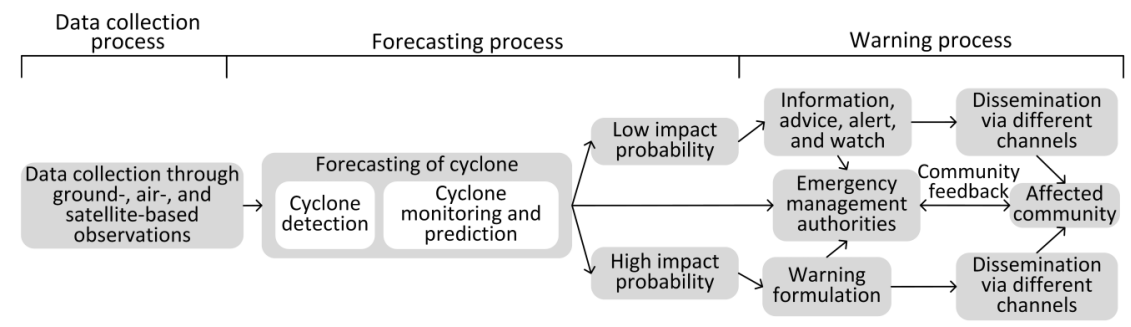

Figure 2-4. Technical processes involved in TC forecasting and warning.

\subsubsection{Data collection}

The availability of necessary data in time is important for producing accurate TC track and intensity forecasts. Data used for forecasting TCs are usually collected using ground-, air-, and space-borne measurement instruments. Ground-based stations are generally known as synoptic weather stations. Observations of standard meteorological parameters like, temperature, wind speed, wind direction, air pressure at sea level, and amount of rainfall can be obtained from these stations on six-hourly basis. Ground-based radars are used to locate clouds and calculate their motion within a certain distance from the radar's location. Another ground-based instrument, vertical wind profiler is capable of providing continuous measurements of wind speed and direction at different tropospheric levels. Information collected using radar and vertical wind profiler is considered significant for both TC track and intensity forecasting.

Airborne instruments, such as weather balloons, aircrafts, and dropwindsondes provide wind speed, wind direction, air temperature, and humidity measurements from various atmospheric levels, which are very important for TC forecasting. Though aircrafts can provide precise information about the development, intensity change, and track of a TC, these observations are mainly available in the Atlantic basin (Aberson et al., 2010; Gray, Neumann, \& Tsui, 1991; Martin \& Gray, 1993).

TCs usually develop and spend most of their lifecycles over the tropical waters; therefore, meteorological information recorded using the available ground-based measurement stations seem not sufficient for precise forecasting of TC tracks and intensity. Before the 1960s, when there were no satellites for observing weather conditions from the space, meteorologists had to rely exclusively on ground-based and upper-air observations for monitoring and forecasting of TCs. 
Since 1960, when the first weather satellite TIROS-1 was lunched, the satellite-derived information of the atmosphere started to play a more and more important role in TC monitoring and forecasting (Saltzman, 1985). The ability to record images over the ocean, good spatial coverage and the frequent availability of new images have rendered satellitederived information as one of the most useful predictors for TC track and intensity forecasting (Bedka, Velden, Petersen, Feltz, \& Mecikalski, 2009; Deb, Kishtawal, \& Pal, 2009; Mueller, DeMaria, Knaff, Kossin, \& Vonder Haar, 2006; Sears \& Velden, 2012; Zhang, Li, Weng, Wu, \& Xu, 2007; Zhang, Xiao, \& Fitzpatrick, 2007) as well as for monitoring other natural disasters, such as tsunamis that create flooding in the coastal areas (Lin, Zhu, \& Sookhanaphibarn, 2012a, 2012b).

The data used for TC track and intensity forecasting are recorded by both polar orbiting (that circle the Earth in an almost north-south orbit, passing close to both poles by maintaining an altitude $\sim 700 \mathrm{~km}$ ) and geostationary satellites (circles the Earth in a geosynchronous orbit by maintaining an altitude $\sim 36,000 \mathrm{~km}$ ). Geostationary meteorological satellites are capable of capturing images of a fixed portion of the earth on a more frequent basis (every few minutes) compared to the polar orbiting satellites; therefore, TCs can be monitored properly throughout their lifecycles using geostationary-satelliterecorded images. Polar orbiting satellites in contrast, orbit the earth continuously and provide a wider variety of atmospheric data and cloud images compared to geostationary satellites but on a less frequent basis (Halliwell, Shay, Jacob, Smedstad, \& Uhlhorn, 2008; Kidder et al., 2000; Reynolds, Rayner, Smith, Stokes, \& Wang, 2002; C. Velden et al., 2005).

\subsubsection{TC track forecasting}

After formation, TCs usually travel hundreds of kilometers over the tropical waters before making a landfall or decaying over the sea. The course that a TC maintains throughout its lifespan is known as its track. A wide range of external and internal forces can influence the track of a TC at any stage of its lifecycle (see section 2.2). Among the external factors influencing TC track, the large-scale environmental currents and the boundary layer conditions, such as air-surface friction and air fluxes that arise near the ocean surface are most significant (Jeffries \& Miller, 1993; Neumann, 1992; Sampson, Jeffries, \& Neumann, 1995). Factors associated with a TC itself constitute the internal factors and these factors include irregularities in the convection process, the beta effect (variation of the Coriolis force with latitude), wind and heat circulations within the TC vortex, intensity, and instability of the outflow layer (Bin et al., 1999; Sampson, Jeffries, \& Neumann, 1995).

Track forecasting techniques use statistical or mathematical equations to relate changes in the current TC's motion characteristics with: (a) changes in the factors governing TC motion, and/or (b) recent-past motion characteristics of the current TC, and/or (c) motion characteristics of historical TCs in the same basin (Aberson, 1998; Jeffries, Sampson, Carr III, \& Chu, 1993; Rhome, 2007; Roy \& Kovordányi, 2012, 2015; Sampson, Jeffries, \& Neumann, 1995). As the vortex of a TC usually extends high in the atmosphere; therefore, information collected from various vertically arranged pressure levels (steering information) are used for track forecasting in addition to the surface-level information (Brand, Buenafe, \& Hamilton, 1981; Ngan \& Chan, 1995; Sampson, Jeffries, \& Neumann, 1995). 
TC track forecasting techniques differ mainly in terms of: (a) which type of predictors are used (Roy \& Kovordányi, 2012; Sampson, Jeffries, \& Neumann, 1995), and (b) which equation systems are used for producing forecasts (Jeffries et al., 1993; McBride \& Holland, 1987; Roy \& Kovordányi, 2012, 2015). On the basis of equation system use, TC track forecasting techniques can be divided into three major groups (Rhome, 2007; Roy \& Kovordányi, 2012): (a) statistical, (b) numerical, and (c) statistical-numerical. Among these three groups of techniques, numerical techniques are the most advanced and commonly used for forecasting TC tracks. Examples of well-known numerical TC track-forecasting techniques include the Met Office (UKMET) model, the European Centre for MediumRange Weather Forecasts (ECMWF) Ensemble Prediction System (EPS), the Hurricane Weather Research and Forecast (HWRF) model, and the Japanese Meteorological Agency (JMA) Ensemble Prediction System (EPS). However, TC track forecasting accuracy of various techniques depends on both quality of the predictors, and the equation systems' ability of translating data into track forecasts (Bin et al., 1999; Jeffries et al., 1993; McBride \& Holland, 1987; Roy \& Kovordányi, 2012).

The Met Office model (UKMET) is a non-hydrostatic global model that works with 70 vertical levels. Data from the operational forecast centers around the globe, from conventional observations, and 6-hour old TC movement characteristics are utilized to extract information about the current TC, which are then initialized in the UKMET model. The model finally produces wind profiles surrounding the TC at lower tropospheric level. This model is run four times a day, twice for producing short-term $(<48 \mathrm{~h})$ and twice for producing very-long-term $(144 \mathrm{~h})$ forecasts. TC track forecasts are produced for all active TCs twice a day during the very-long-term model run (Chan \& Kepert, 2010). Parameterizations and grid configuration of the model as well as schemes that have been introduced to this model with a view to enhance the model's prediction ability (Chan \& Kepert, 2010) are described in Table 2-1.

Table 2-1. The Met Office (UKMET) model: parameterization, grid configuration, and modification.

\begin{tabular}{|c|c|}
\hline Model properties & Description \\
\hline Parameterizations & $\begin{array}{l}\text { - The Edwards-Slingo radiation scheme } \\
\text { - Convection with CAPE closure } \\
\text { - Momentum transports and convective anvils, } \\
\text { - Gravity-wave drag scheme and MOSES (Met Office } \\
\text { - Surface Exchange Scheme) surface hydrology } \\
\text { - Soil model scheme }\end{array}$ \\
\hline \multirow[t]{2}{*}{ Grid configuration } & $\begin{array}{l}\text { Grid size: this model has a semi-Lagrangian advection } \\
\text { scheme using } 1024 \text { x } 769 \text { grid points. }\end{array}$ \\
\hline & $\begin{array}{l}\text { Grid resolution: the horizontal grid resolution of the } \\
\text { model is } 0.3515625^{\circ} \text { latitude } x 0.234375^{\circ} \text { longitude } \\
\text { (about } 25 \mathrm{~km} \text { at mid latitude). }\end{array}$ \\
\hline \multirow{3}{*}{$\begin{array}{l}\text { Important modifications } \\
\text { since the model became } \\
\text { operational }\end{array}$} & In 1994, TC initialization scheme was introduced. \\
\hline & $\begin{array}{l}\text { In 2004, a 4D-var data assimilation scheme was } \\
\text { introduced (Rawlins et al., 2007). }\end{array}$ \\
\hline & $\begin{array}{l}\text { In 2007, a new sea-surface temperature and sea ice } \\
\text { scheme (OSTIA) was introduced (Stark, Donlon, } \\
\text { Martin, \& McCulloch, 2007). }\end{array}$ \\
\hline
\end{tabular}


The European Centre for Medium-Range Weather Forecasts (ECMWF) Ensemble Prediction System (EPS) functions through a set of diagnostic and prognostic equations. The diagnostic equations handle the static relationship between pressure, density, temperature, and height. The prognostic equations in contrast, deal with the time evolution of the horizontal wind components, surface pressure, temperature and the water vapor contents of an air parcel (Persson, 2013).

ECMWFEPS uses 51 singular-vector-generated perturbed initial conditions to produce 51 ensemble members (Buizza, Milleer, \& Palmer, 1999; Molteni, Buizza, Palmer, \& Petroliagis, 1996; Richardson, 2000). Model parameterization, grid configuration, and modifications for improving prediction accuracy are described in Table 2-2.

Table 2-2. ECMWFEPS: parameterization, grid configuration, and modifications.

\begin{tabular}{ll}
\hline \multicolumn{1}{c}{ Model properties } & \multicolumn{1}{c}{ Description } \\
parameterization & $\begin{array}{l}\text { Used to include the influences of radiation, gravity } \\
\text { wave drag, vertical turbulence, convection, clouds and } \\
\text { surface interaction. }\end{array}$ \\
\hline Grid configuration & $\begin{array}{l}\text { Horizontal: both grid point and spectral coordinates } \\
\text { are used to representations the data on the horizontal } \\
\text { plane (Persson, 2013). The model is run using } 32 \mathrm{~km} \\
\text { and 64 km horizontal resolutions to produce forecasts } \\
\text { for 0-10 days and 10-32 days respectively. }\end{array}$ \\
\cline { 2 - 2 } & $\begin{array}{l}\text { Vertical: currently the model has 91 vertical levels, } \\
\text { where spacing between two vertically arranged layers } \\
\text { is finest near the planetary boundary and coarsest near } \\
\text { the model top. }\end{array}$ \\
\hline Important modifications & $\begin{array}{l}\text { In 2002, moist tropical singular vectors were } \\
\text { introduced, to include the effects of diabatic processes } \\
\text { operational }\end{array}$ \\
& $\begin{array}{l}\text { in the TC formation basins (Barkmeijer, Buizza, } \\
\text { Palmer, Puri, \& Mahfouf, 2001). }\end{array}$ \\
\hline
\end{tabular}

ECMWFEPS is run twice a day and does not use any specific initialization of tropical cyclones. However, research has been carried out to investigate the impact of using different kinds of data and of assimilation methods on TC forecasts. For example, Isaksen (1997) shows that the ECMWF 4D-Var data assimilation system (Isaksen, 1997) improves the analyses of tropical cyclones compared to previous assimilation procedures (Isaksen \& Janssen, 2004; Isaksen \& Stoffelen, 2000).

The Hurricane Weather Research and Forecast (HWRF) model was developed by NCEP with a view to address the problems related to hurricane forecast in the Atlantic and the Northeast Pacific basins (Chen, Zhao, Donelan, Price, \& Walsh, 2007; C. Davis et al., 2008; Gopalakrishnan et al., 2011). HWRF became available for operational use in 2007 and is run four times a day (Tallapragada et al., 2014). Model parameterization, grid configuration, and major modifications done to the model are described in Table 2-3.

Table 2-3. HWRF: parameterization, grid configuration, and modifications.

\begin{tabular}{ll}
\hline Model properties & \multicolumn{1}{c}{ Description } \\
\hline Parameterization & $\bullet$ Microphysics parameterization \\
processes & $\bullet$ Cumulus parameterization \\
& $\bullet$ Surface-layer parameterization \\
\hline
\end{tabular}




\begin{tabular}{|c|c|}
\hline Model properties & Description \\
\hline & $\begin{array}{l}\text { - Planetary boundary layer parameterization } \\
\text { - Atmospheric radiation parameterization }\end{array}$ \\
\hline \multirow[t]{2}{*}{ Grid configuration } & $\begin{array}{l}\text { Horizontal: HWRF holds its predictors in a multiple } \\
\text { movable two-way interactive nested grid system that } \\
\text { follows the projected path of the storm. The outer grid } \\
\text { extends over a } 75^{\circ} \times 75^{\circ} \text { area and has a resolution of } 18 \mathrm{~km} \text {. } \\
\text { The intermediate grid has higher resolution (around } 6 \mathrm{~km} \text { ), } \\
\text { and the innermost grid operates at } 2 \mathrm{~km} \text { horizontal } \\
\text { resolution. }\end{array}$ \\
\hline & $\begin{array}{l}\text { Vertical: HWRF uses sigma-pressure hybrid coordinates as } \\
\text { vertical coordinates and currently characterized by } 42 \\
\text { vertical levels. }\end{array}$ \\
\hline \multirow{2}{*}{$\begin{array}{l}\text { Important } \\
\text { modifications since } \\
\text { the model became } \\
\text { operational }\end{array}$} & $\begin{array}{l}\text { To improve the TC track and intensity forecast, a triple } \\
\text { nesting of the simulation grid was introduced in } 2012 \text {. }\end{array}$ \\
\hline & $\begin{array}{l}\text { To increased horizontal resolution, vortex initialization } \\
\text { scheme and preprocessing system have been upgraded in } \\
2015 \text {. }\end{array}$ \\
\hline
\end{tabular}

The Japanese Meteorological Agency (JMA) Typhoon Ensemble Prediction System (Typhoon EPS) was developed specially for TC forecast. This model is characterized by a horizontal resolution of $\sim 55 \mathrm{~km}$ and 60 levels in the vertical. A singular-vector approach is used to generate 11 initial conditions to produce 11 ensemble members, of which one is non-perturbed and the remaining ten are perturbed. The model is run four times a day, when TCs form in the responsibility area of the Regional Specialized Meteorology Center (RSMC), Tokyo $\left(0^{\circ} \mathrm{N}-60^{\circ} \mathrm{N}, 100^{\circ} \mathrm{E}-180^{\circ} \mathrm{E}\right)$ or TCs formed outside may move into the area within the next 24 hours. Typhoon EPS is capable of producing forecasts for the next 5 days.

\subsubsection{TC Intensity forecasting}

TC intensity is a measure of the maximum sustained wind speed at or near the surface surrounding a low-pressure center or the minimum sea-level pressure at the center of the TC vortex. Within a TC, the highest wind speed is usually observed near the center (eye) (Sampson, Jeffries, Neumann, et al., 1995), which occupies a small area of the whole lowpressure system. As it is unlikely that a TC passes directly over a measurement instrument (ocean buoy or instruments placed on ships), direct pressure and wind speed measurements within the TC are difficult to achieve. Therefore, forecasters rely on the data provided by vertical wind profilers, radars, and/or satellites for indirect estimation of wind speed and pressure within a TC.

Similarly to track forecasting, persistence, climatology, and steering predictors are used for TC intensity forecasting. Statistical and mathematical equations are also used for producing TC intensity forecast on the basis of these predictors. However, of the data recorded by various instruments, the use of satellite images (both geostationary and polar orbiting) is common in TC intensity forecast. Several TC intensity forecasting techniques have also been developed on the basis of these satellite-recorded images (Dvorak, 1972, 1975, 1984, 1995; Gentry, Rodgers, Steranka, \& Shenk, 1980; Le Marshall, Leslie, Abbey 
Jr, \& Qi, 2002; Olander \& Velden, 2007; Rodgers, Mack, \& Hasler, 1983; C. Velden et al., 2006).

The concept of using satellite images for successful estimation and forecasting of TC intensity was first introduced by Dvorak (Dvorak, 1972). He used visible and infrared channel images to extract specific cloud patterns associated with a TC and then related them with deepening or weakening of the TC (Figure 2-5). Several techniques use Dvorak's empirical rules for automatic detection of TC intensity on the basis of satellite images (Olander \& Velden, 2007; C. S. Velden, Olander, \& Zehr, 1998). For example, automated TC intensity forecasting techniques developed by Velden and coauthors (1998) and Olander and Velden (2007) follow the basic concepts and empirical rules proposed by Dvorak. There are also TC intensity forecasting techniques that use satellite images as the only predictor, but these techniques do not use Dvorak's empirical rules (Bankert \& Tag, 2002; Gentry et al., 1980). Examples of these techniques include satellite-measured equivalent blackbody temperature developed by Gentry and coauthors (1980), and an automated intensity estimation technique developed by Bankert and Tag (2002).
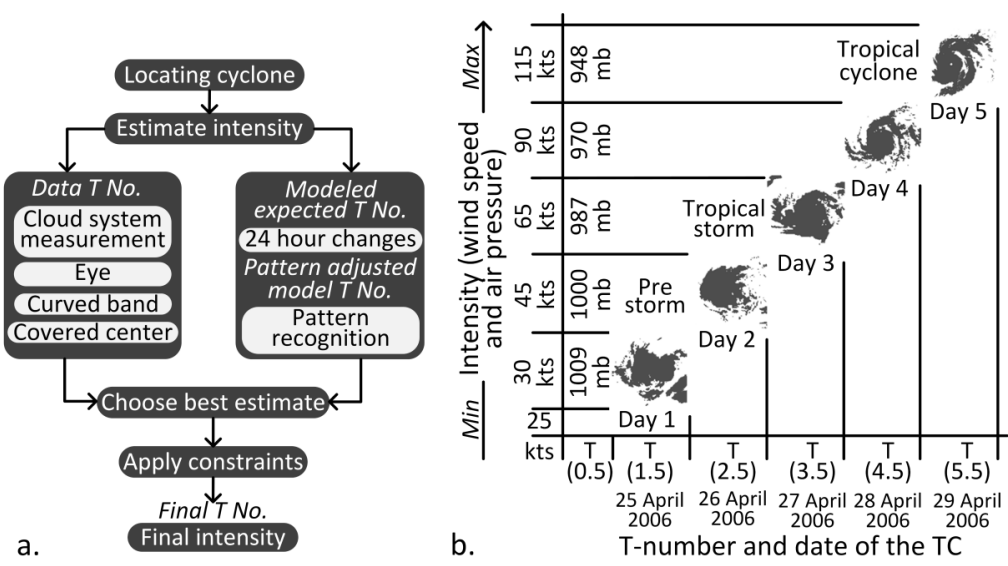

Figure 2-5. Cyclone intensity forecast technique developed by Dvorak (Dvorak, 1975, 1984): a. basic steps in TC intensity forecasting, and b. forecasted intensity level of TC Mala using Dvorak's technique.

\subsubsection{Warning message formulation and dissemination}

Warning message formulation and dissemination is the last technical process of a TC early warning system. Informative and understandable warning message is important for eliciting expected response to evacuation orders from the residents living in the coastal areas. Which part of the population should be warned, and the residents from which areas should be evacuated can best be determined on the basis of accurate forecasting of TCs. The damages caused by TCs can be reduced considerably, if accurate and informative warnings are delivered to emergency management authorities and to the residents in the probable affected areas in time (Islam et al., 2004; B. K. Paul, 2009, 2012; B. K. Paul \& Dutt, 2010; Sahni \& Ariyabandu, 2004). 
A warning message is a mechanism by which threats imposed by an approaching disaster is transferred from the warning message providers to the warning message receivers (Sahni \& Dhameja, 2004; Zschau \& Küppers, 2003). In cyclone emergencies, effectiveness of a warning message is very important. Formulation of the warning message should be done in a way, which is expected to enable the recipients to act as quickly as possible, take appropriate actions, and to be maximally prepared under the given circumstances (I. Davis et al., 1998; Parker, 1999). This means, wording of the warning message is critical for achieving expected response from the residents (Parker, 1999). To be effective, a warning message should contain a number of components (Brady, 2005; Drabek, 1999; Gladwin et al., 2007; Zschau \& Küppers, 2003):

1. The message should be simple so that anybody can understand it easily.

2. The expected consequences of the imminent cyclone attack and the actions required to successfully handle the forecasted situation should be stated explicitly.

3. Events related to the cyclone, like surge levels, should be given special attention.

4. The most important information should be placed in the beginning of a message.

5. Location references should be made in relation to well-known places.

6. An easily understandable language should be used (e.g., for specifying time, it is better to use a.m. and p.m. instead of a 24-hour clock).

7. A statement of recommendations on possible preparative actions should be included.

After formulation, the warning message is sent to the emergency management authorities and is disseminated through different media so that the residents in the threatened areas can receive it. A reliable dissemination system is another vital component in the warning process (Parker, 1999). To achieve improvements in early warning, the local and the national communication channels must be capable of providing rapid, reliable, and consistent means for disseminating warnings to the threatened communities. These channels could be general like radio, television, news bulletins, and newspapers, or specific like, telephone calls, short message service, emails, fax, and megaphone announcements. The choice of channels depends primarily on the availability of different options but is also determined by the warning lead time, the rate at which a TC is changing its track, intensity etc., and whether there is a need for specific or more general means for dissemination (I. Davis et al., 1998; Joseph, 1994).

\subsection{Community response to warning}

In disaster management terminology, the human action upon receiving a warning is called a response. An important point is that even if a $\mathrm{TC}$ is forecasted and warning messages are delivered to the threatened communities well before the TC makes a landfall, people living in the threatened communities may not respond to the disseminated warning messages as expected. This highlights that the success of an early warning system depends critically on the community's response to warnings. In practice, recipients of the warning messages do not respond to it directly as individual persons; instead, an individual's response emerges in the context of interaction with other people. 
What we elicit as response from the individuals living in a community is mostly a group or collective product rather than an individual's reaction (Quarantelli, 1990). Therefore, the community's response to a cyclone warning is a function of both selective perception and social confirmation. It is impossible to overlook the fact that providers' and receivers' perceptions of the warning message may not always be the same. This means, warning message recipients living in a community may not always respond to the message as expected (Drabek, 1999).

\subsubsection{Incorporation of human perception into cyclone warning}

The concept of incorporating residents' perception into disaster emergency management is not new. Social scientists started to do research on disaster management in the 1960s, with many of the early studies focusing on disaster warning and how individuals and communities respond to it (McLuckie, 1970; Quarantelli, 1990). Later, most notably during the period of 1990 to 2000, the necessity of incorporating community perception was brought up again worldwide under the International Decade for Natural Disaster Reduction (IDNDR) (Boullé, 1999).

Throughout the 1990's, better access to forecasts and greater effectiveness of early warning have been given substantial emphasis as a means to reach IDNDR objectives (Boullé, 1999): (a) reducing human casualties and property damage, and (b) dealing with social and economic problems caused by disasters especially in the developing countries. The success of a disaster forecasting and warning system depends not only on scientific and technical abilities of hazard identification and forecasting but also on proper understanding of community's view of risk and community's active participation in the early warning system (ABM (Australia Bureau of Meteorology), 2009). IDNDR has worked to promote this understanding of disaster forecasting and warning and to establish the importance of incorporating socio-economic dimensions into forecasting and warning (K. S. Khan, 2005).

People living in the community have their own view of risk and on the basis of that view they make decisions to protect themselves from TC attacks (Alam \& Collins, 2010). This entails that residents' response to warning cannot be understood properly without having a better understanding of their perception of risk (Zschau \& Küppers, 2003). Considering these research findings, disaster researchers have put effort into incorporating human perception into cyclone warning systems. These efforts resulted in considerable success in reducing the damage to both life and property caused by TCs (B. K. Paul, 2009, 2012; B. K. Paul \& Dutt, 2010; Thomalla \& Schmuck, 2004).

A community's response to warning is largely governed by the community's view of risk, which might be different from the outsiders. Therefore, outsiders might find it very difficult to understand how people living in a community perceive risk and respond to warnings. There are several reasons for this difficulty (Zschau \& Küppers, 2003):

1. People living in a community and disaster researchers may hold completely different views of a potential disaster.

2. The way of measuring and describing risk, as well as the concept of risk differs between these groups.

3. Disaster researchers' attitude could also pose a problem. If they have a preconception that their understanding of risk is better than that of the people living in a community. 
To overcome these difficulties, it is necessary to have a deeper understanding of the vulnerabilities that a particular community is exposed to and understanding risk from the community's point of view (Douglas \& Wildavsky, 1983; Gladwin et al., 2007). Once the causes of vulnerability to TCs and the community's perception of risk are properly understood, it becomes easier to identify the problems people usually face to follow the warnings (Dash \& Gladwin, 2007; B. K. Paul \& Dutt, 2010).

\subsection{Cyclone early warning system in Bangladesh}

The Storm Warning Center (SWC), a specialized unit of BMD (Bangladesh Meteorological Department), is responsible for forecasting and issuing warnings for TCs in Bangladesh. BMD collects meteorological data through 35 ground-based, 10 weather balloon, 5 radar, and 3 rawinsonde stations. BMD additionally receives weather satellite data, meteorological and sea surface data over the BoB (Bay of Bengal) collected through ocean buoys as well as numerical model generated weather forecast from other national and regional meteorological offices as a member state of world meteorological organization (Obasi, 1994; (RSMC (Regional Specialized Meteorology Centers), New Delhi, 2013); Zschau and Küppers, 2003).

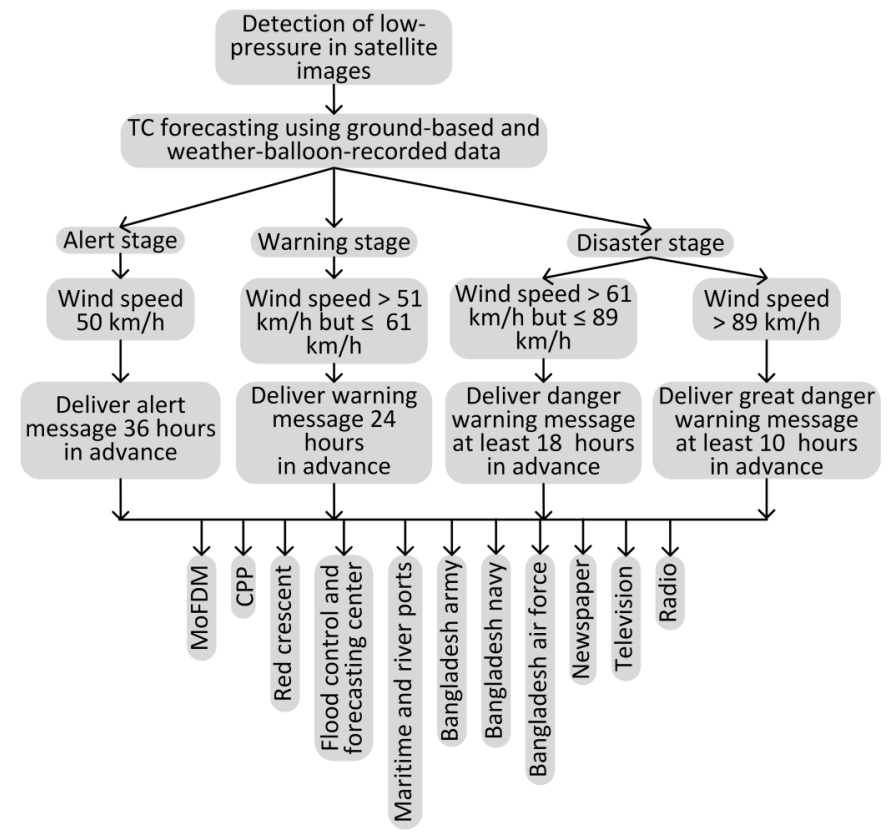

Figure 2-6. TC forecasting and warning dissemination process at BMD.

Prepared based on Chowdhury (2002), Debsarma (2001), and Miyan (2006).

BMD uses two forecasting techniques: Storm Track Prediction (STP) and Steering and Persistence (STEEPER) for forecasting TCs (ADRC (Asian Disaster Reduction Center), 2005; Debsarma, 1999). Quadratic regression equations constitute the computational base for STP. The future positions of the current TC are forecasted on the basis of the observed track of historical TCs having a similar movement path. As the position of a huge 
phenomenon like a TC cannot be represented correctly using a single point fixed at the storm center, forecasts produced by STP may not always be accurate.

STEEPER in contrast, uses two types of information for forecasting TCs: (a) a series of time tagged TC locations, and (b) wind information near the sea surface. A Kriging interpolation technique is applied to this data to compute the future position of a TC. Despite this effort, inaccurate predictions in terms of TC intensity and movement, landfall timing, intensity upon impacts, and height of the accompanying surge during TC landfall, has often been criticized (C. E. Haque \& Blair, 1992; Hossain, Islam, Sakai, \& Ishida, 2008; Miyan, 2006). Once forecasts are produced, BMD sends warnings to the maritime and river port authorities, relief and rehabilitation authorities, local level administrative officials, public media for dissemination and to the cyclone preparedness program (CPP), and non-governmental organizations (Figure 2-6) (Chowdhury, 2002; Debsarma, 2001; Miyan, 2006).

In recent years, there have been a considerable improvement in cyclone warning dissemination mainly due to development in the area of information and communication technology, especially internet, mobile phones and improved broadcasting technology with global television channels (Hossain et al., 2008). Though significant improvements have been achieved in cyclone warning dissemination in Bangladesh, quality of the warning messages has not been improved much in last decades (C. E. Haque \& Blair, 1992; Hossain et al., 2008; Tatham, Spens, \& Oloruntoba, 2009). Comparison between the earlier study conducted after the great Bhola cyclone in 1970 (Frank \& Husain, 1971) and study carried out after Cyclone Sidr in 2007 (Hossain et al., 2008) reveals that BMD's TC forecasting performance has not been improved much during the last several decades. Moreover, the existing warning system is not easy to understand and even sometimes incomprehensible to educated people as well (C. E. Haque \& Blair, 1992; Miyan, 2006). Moreover, residents are not conscious of the implications of different signal numbers; nor are they aware of the different signals for maritime and river ports (Appendix B and Appendix C). Therefore, actual information contained in the warning message cannot be conveyed to the residents living in the coastal areas (Miyan, 2006).

\subsection{TC track and intensity forecasting using ANN}

Processing of information through interconnected groups of artificial neurons (units) assigned to different layers or layers of units constitute the base for information processing in an Artificial Neural Networks (ANN). ANN is a parallel adaptive system, which can be used to model complex relationships between inputs and outputs to describe existing as well as to reveal new patterns in the analyzed datasets (Kim \& Yum, 2004; Schalkoff, 1997). Once a valid network is adequately trained, it can make predictions on new set of data, not used for training the network. Development of ANNs requires an understanding of both the principles of neural networks and the scientific field within which they are to be applied (Dam \& Saraf, 2006). Four basic components are necessary to construct a conventional ANN (Schalkoff, 1997; Villmann, Merényi, \& Hammer, 2003):

1. Units, which are arranged in layers

2. Connections between the layers

3. Weights associated with the connections, and

4. A procedure for defining the output from each unit on the basis of input. 
Conversely, computation using ANNs involve three components (Kitano, 1994): (a) selecting and preparing the data, (b) architecture of the network and activation functions, and (c) selection of a training method along with synaptic weights and biases. In an ANN, each unit receives and processes one/multiple input signals received from the previous layer (feedforward), performs some computations using an activation function, and finally outputs the result (Cartwright \& Kharma, 2008).

It has previously been mentioned that satellites became one of the most important instruments for collecting information about TCs and their surrounding environment. Though the operational numerical TC forecasting techniques are capable of handling data recorded using various instruments, they are complex, require high-end computers to run, and very much vulnerable to inaccurate initial values (Bin et al., 1999; Chan \& Kepert, 2010; Jeffries et al., 1993; Roy \& Kovordányi, 2012, 2015). These complexities have motivated researchers into advanced and novel approaches to forecasting TCs, in particular, techniques using ANNs. ANNs are characterized by the following important features (Villmann et al., 2003):

1. Adaptivity: ability to change the internal representation such as connection weights, network structure if new information is available.

2. Robustness: handling of missing, noisy, or confused data.

3. Power/speed: handling of large data volumes in acceptable time due to inherent parallelism.

4. Non-linearity: the ability to represent non-linear functions or mappings.

These properties render ANNs useful and efficient for analyzing satellite data for TC track and intensity forecasting as well as for analyzing factors governing TC track and intensity (Aguilar-Martinez \& Hsieh, 2009; A. Wu, Hsieh, \& Tang, 2006).

Among various machine-learning algorithms, ANNs are well known for TC track and intensity forecasting (Ali, Kishtawal, \& Jain, 2007; Jin, Yao, \& Huang, 2008; Pickle, 1991). In addition to the inherent characteristics, ANN-based TC forecasting techniques are inexpensive in terms of computational resource requirements. ANN-based techniques are particularly advantageous in the following sense:

1. Satellite images used as inputs to ANNs are widely available and mostly free of cost.

2. These techniques can be run on standard PCs, whereas almost all numerical TC forecasting techniques require huge computational resources (super computers).

3. Though considerable time is required for training a network, the trained network is capable of producing TC track and intensity forecasts in a few minutes or in fractions of a minute.

4. ANN techniques are capable of producing good accuracy TC track and intensity forecasts (Ali et al., 2007; Feng \& Liu, 2004; Jin et al., 2008; Lee \& Liu, 2000; Pickle, 1991).

\subsection{Biologically based ANN techniques for image processing}

The approach of using biologically based machine learning for recognizing objects in visual images is well-known (Fukushima, 1980, 1988, 2013; Fukushima \& Miyake, 1982; Hubel 
\& Wiesel, 1965, 1977, 1998; Itti \& Koch, 2001; Itti, Koch, \& Niebur, 1998; LeCun, Bengio, \& Hinton, 2015; LeCun, Bottou, Bengio, \& Haffner, 1998; LeCun, Huang, \& Bottou, 2004; Li \& Itti, 2011). Parallel feature extraction across the image, as well as stepwise hierarchical combination of the extracted features into task relevant patterns constitute a reliable basis for visual input interpretation in biologically based neural networks. Irrespective of the features' complexity, the human visual system can naturally recognize complex features and achieve position, size, and rotation invariances at the same time. However, achieving position, size, and rotation invariances in object recognition using biologically inspired ANNs is still considered to be difficult. The more well-known ANN techniques using this approach for image processing are described in the next three subsections.

\subsubsection{Neocognitron}

Neocognitron is a biologically inspired hierarchical multilayer ANN proposed by Fukushima (1980). Fukushima designed the architecture of neocognitron on the basis of Hubel and Wiesel's $(1965,1977)$ hypothesis of the visual nervous system. The connections between the hierarchically arranged layers are feedforward. The first two layers of the neocognitron (input and contrast extraction) simulate the photoreceptors in the retina and the Lateral Geniculate Nucleus (LGN) (Fukushima, 1980; Fukushima \& Miyake, 1982). After the contrast extraction layer, pairs or layers represent the remaining processing levels in the hierarchy. One layer of each pair consists of simple cells (S-cells) that extracts features from their corresponding Receptive Fields (RFs), whereas the other layer of each pair contains complex cells (C-cells) that tends to achieve invariances by reacting to the shapes uniformly in the hierarchy (Fukushima, 1980, 1988). These processing levels correspond to the visual information processing areas, V1 (primary visual cortex) $\rightarrow \mathrm{V} 2 \rightarrow$ $\mathrm{V} 4 \rightarrow$ and IT (inferior temporal cortex) in the human brain, where simple features (oriented line segments) are primarily extracted from the inputs and then integrated into increasingly complex features. Therefore, a cell located higher up in the processing hierarchy, usually has a large RF and is less sensitive to the feature's location in the input.

The neocognitron can be trained using both supervised and unsupervised learning rules. Unsupervised learning constitutes the learning rule for the early versions of neocognitron (Fukushima, 1980; Fukushima \& Miyake, 1982), which allows the network to achieve selforganizing capability. These versions are not effective in recognizing differences between characters with similar shape. To deal with this problem, Fukushima improved the neocognitron by adding a supervised learning capability to it (Fukushima, 1988).

In the most recent version of neocognitron, if a group of postsynaptic S-cells remains inactive despite non-zero inputs from their RF, a new S-cell is generated and added to the network to learn that particular input pattern. Here the strength of the connection to the newly generated S-cell is proportional to the response of the presynaptic C-cells at that moment and remains unchanged regardless of varying degree of stimuli given to the network during training (Fukushima, 2013). This newly introduced learning rule is called add-if-silent. According to Fukushima, this learning rule makes neocognitron computationally effective and learning process simpler and stable compared to the previous versions. 


\subsubsection{Convolutional Neural Networks}

Convolutional Neural Networks (CNNs) have their roots in Fukushima's neocognitron (LeCun et al., 2015). CNNs have a hierarchical structure and use feedforward connectivity with a backpropagation of error. For detecting features in the input image, CNNs combine three architectural ideas: local RFs, weight sharing (weight replication), and spatiotemporal subsampling (LeCun \& Bengio, 1995; LeCun et al., 1998).

The idea of connecting a unit to a local RF in the input is rooted in Hubel and Wiesel's discovery of locally-sensitive visual neurons (Hubel \& Wiesel, 1965). In a CNN, units with local RFs extract simple features (oriented edges, end-points) from the input image, which are then combined into increasingly complex features in the successive hierarchical levels (LeCun et al., 1998). Low-level feature detectors, which are useful for a part of the input image, are expected to be equally useful for other parts of the input image. This idea is implemented by forcing a set of units (have their RFs located in different parts of the input image) to have identical weights (weight sharing) (LeCun \& Bengio, 1995; LeCun, Bottou, \& Bengio, 1997; LeCun et al., 1998). Units having the same weights are then organized in a plane and outputs of the units in a plane constitute a feature map. Likewise, units with different weights are organized in different planes and generate different feature maps by performing the same operation on different parts of the image. RFs of neighboring units in a feature map are centered on corresponding neighboring units in the previous layer; as a result, RFs of neighboring units overlap with each other. When a feature map is employed for scanning the input image, a single unit with a local RF scans the image and stores the extracted information at corresponding location in the feature map. This operation is performed sequentially for all the units in a feature map as well as for other units in all the feature maps (LeCun et al., 1998).

When a feature is detected, the feature's precise location in the input image is not considered to be important any more but its gross position relative to other features in the input becomes relevant. This is because; precise location of each of the identified features is irrelevant for recognizing the pattern (as patterns associated with different inputs are likely to be different). To reduce the precision of the identified features' position coded in a feature map, spatial resolution of the feature map is reduced. This is achieved by using subsampling layers, which perform a local averaging and a subsampling to reduce the spatial resolution of the feature map and to reduce the sensitivity of the output to position shifts and misrepresentations (LeCun et al., 1997, 1998; Taylor, Fergus, LeCun, \& Bregler, 2010).

LeCun and coauthors have successfully implemented these networks for optical character recognition (LeCun et al., 1990, 1997, 1998; LeCun \& Bengio, 1995). CNNs are also widely used for processing other types of image, such as face recognition (Fasel, 2002; Garcia \& Delakis, 2004; Lawrence, Giles, Tsoi, \& Back, 1997), medical image processing (Lo et al., 1995), and traffic sign recognition (Cireşan, Meier, Masci, \& Schmidhuber, 2011; Sermanet \& LeCun, 2011).

CNNs process input images at more than three hierarchical levels (LeCun \& Bengio, 1995; LeCun et al., 1998), which is the most basic property of a deep learning neural network (LeCun et al., 2015). However, the term Deep Neural Networks (DNNs) is also used to indicate a version of CNN, where subsampling layers have been replaced with maxpooling layers (Cireşan, Meier, Masci, Gambardella, \& Schmidhuber, 2011; Cireşan, Meier, Masci, \& Schmidhuber, 2012; Cireşan, Meier, \& Schmidhuber, 2012; LeCun et al., 
2015). Though both subsampling and max-pooling layers play the same role, the way they play the role is different. Instead of calculating the local average (subsampling), maxpulling layers consider the maximum activation over non-overlapping rectangular regions (Cireşan, Meier, Masci, et al., 2012; Krizhevsky, Sutskever, \& Hinton, 2012; LeCun et al., 1998).

\subsubsection{Saliency based visual attention models}

Saliency based models have a scene-based (bottom-up) hierarchical structure (Itti et al., 1998; Koch \& Ullman, 1987), which is similar to the biologically plausible architecture proposed by Koch and Ullman (1987). Feedforward mechanism is used for extracting features from the input images (Borji \& Itti, 2014; Itti et al., 1998). A form of feedback is also used to filter out image features at lower levels, which are uninteresting for the task (Itti et al., 1998; Wolfe, 1994).

These models extract features from the input image and recognize the extracted features in a way, which is comparable to the pattern recognition process in CNNs. In these models, input images are primarily decomposed into a set of topographic feature maps. These topographic feature maps are computed using a set of linear center-surround operations, which bear a resemblance to RFs in the human visual system (Itti et al., 1998; Zhao \& Koch, 2013). Only the salient spatial location (representing feature that is relevant for the task) within each map is allowed to stand out from their surrounding locations and is fed into the next processing level. At this stage, only the task-relevant image locations are noticeable in the maps, which are then linearly combined to create the final saliency map and location(s) of interest is attended (Itti \& Koch, 2001; Itti et al., 1998; Zhao \& Koch, 2013).

\subsubsection{ANNs used in this research}

The biologically based ANNs used for TC track and intensity forecasting in this research differ from the previously described ANNs mainly in terms of the type of connections between hierarchical processing levels and learning rule (O'Reilly, 1998; O'Reilly \& Munakata, 2000; O’Reilly, Munakata, Frank, Hazy, \& Contributors, 2012). The ANNs used in this research are recurrent. That means, all the hierarchically arranged levels but the inputs are bi-directionally connected, where activations are allowed to go in both bottom-up and top-down directions (Essen, Anderson, \& Felleman, 1992; McClelland, 1993; O'Reilly, 1998; O'Reilly \& Munakata, 2000). These bi-directional connections offer constraintsatisfaction, where both lower- and higher-level constraints can be used at the same time for input image interpretation and processing (O'Reilly, 1998). To be specific, during training, the networks simultaneously try to satisfy a number of constraints imposed on them: inputs from the input layers, the weights, and activation state of the network (O'Reilly \& Munakata, 2000). This capability is particularly advantageous for processing occluded and noisy images. The k-Winners-Take-All (kWTA) inhibitory function plays an important role in the networks' constraint satisfaction performance via restricting the parallel search space for the units, while finding the activation states that would satisfy the constraints optimally (O’Reilly \& Munakata, 2000).

Satellite-recorded TC images are not always complete. Parts of TC images can be missing due to irregularities in the satellite sensor response and/or data recording. With bi- 
directional connectivity, these ANNs are expected to produce good accuracy TC track and intensity forecasts even if partly incomplete images are used for training and testing. Though the most recent version of neocognitron is capable of recognizing occluded letters and restoring occluded shapes (circles, rectangles, triangles) (Fukushima, 2013), processing occluded cloud images seems to be a difficult task to handle using neocognitron.

The backpropagation of error is the most commonly used learning algorithm in the area of satellite meteorology (Brad \& Letia, 2002; Feng \& Liu, 2004; Hong, Hsu, Sorooshian, \& Gao, 2004; Jin et al., 2008; Rivolta, Marzano, Coppola, \& Verdecchia, 2006). All the ANNs described in the previous three subsections use either supervised or unsupervised learning as their learning rules. However, the learning algorithm implemented by the ANNs used in this research is a combination of supervised (contrastive Hebbian learning, a biologically based error driven algorithm, an alternative to backpropagation of error) and unsupervised (model based Hebbian learning) learning (O'Reilly \& Munakata, 2000). By using a combined learning approach, the benefits of both types of learning can be included in the same network. Unsupervised learning helps with adapting the extraction of meaningful features on the basis of the five types of input images used in the current research (see section 4.3.4). Supervised learning is necessary to associate the extracted feature patterns with the correct output movement direction and intensity levels. Performing both of these tasks together would be difficult for any of the learning algorithms individually.

\subsection{Exploratory study on TC movement direction prediction}

An earlier study on TC movement direction forecasting using biologically based ANNs conducted by Kovordányi and Roy (2009) has established the conceptual basis for this research (Kovordányi \& Roy, 2009) (Appendix A). The authors trained and tested a biologically based hierarchical ANN on the basis of NOAA-Advanced Very High Resolution Radiometer (AVHRR) satellite recorded infrared image of TC Mala (Figure 2-7), formed in the BoB (Bay of Bengal) in 2006. Movement direction forecasts produced by the network were only valid for the immediate future.

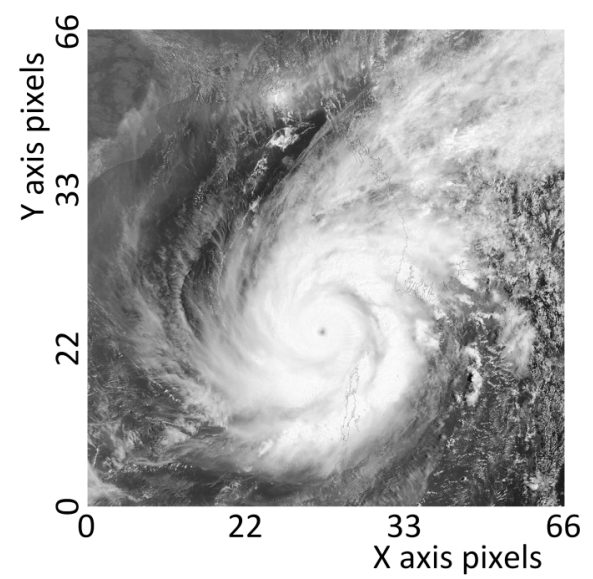

Figure 2-7. NOAA-AVHRR infrared channel image of TC Mala used for forecasting movement direction. 


\subsubsection{Data and method}

The original AVHRR infrared image was cropped carefully so that outer cloud bands containing valuable information about TC's future movement direction were not lost (Figure 2-7). The cropped infrared image was downsized to 66 x 66 pixels. To abstract away from cloud patterns' rotation, size, and position, the image was rotated (clockwise in $45^{\circ}$ increments), each rotated image was zoomed (using 0.8, 1, and 1.2 zoom factors), and finally each rotated and zoomed image was shifted in position within the input frame by zero, one, or two steps in eight directions.

The size of the position shifts was also varied across training sessions to compare the network's performance for the one-pixel and the three-pixel step sizes. This transformation generated 1200 images from the NOAA-AVHRR image of TC Mala. Of the 1200 images, a random subset of approximately $95 \%$ of the images was used for training and the remaining $5 \%$ were used for testing the network.

A mixture of $1 \%$ Hebbian, and otherwise error-driven learning, at the projection connecting the input and the first hidden layer, and a mixture of $0.1 \%$ Hebbian, and otherwise error-driven learning, at the other projections in the network was used. Forecasts were produced into eight movement directions $\left(360^{\circ}\right.$ was divided into eight equal angles). Correct movement direction for the immediate future was primarily determined by visual estimation for the original satellite image and was automatically calculated for the transformed images during processing. A single infrared image and artificially rotated movement direction for that image made an input-output pair used for training and testing the network.

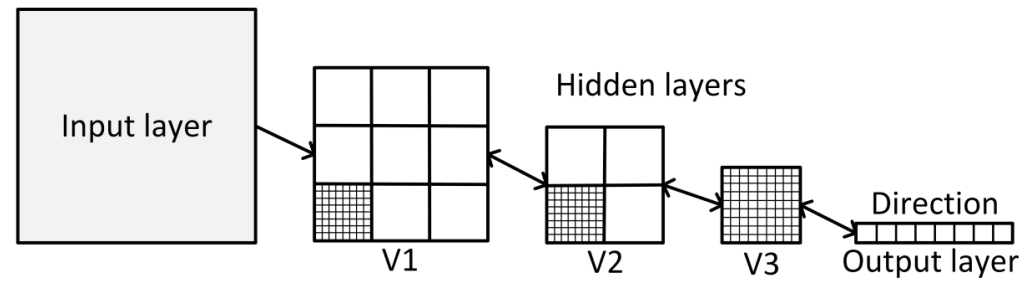

Figure 2-8. The network used for TC movement direction forecast.

The network processed inputs at five hierarchical levels. Of the five levels, the first and the last processing levels were input to and output from the network respectively. Levels between the input and the output, extracted features available in the input images and recombined the extracted features for generating outputs. Therefore, one input layer, three hidden layers (V1, V2, and V3), and one output layer (direction) constituted the basic structure of the track-forecasting network (Figure 2-8). All the layers except the input were bi-directionally connected, which allowed the layers, both to send input to other layers and receive feedback from those layers. Structure of the five layers are described in the list below:

1. The input layer was $66 \times 66$ pixels in size and was divided into nine parts. Each of these parts was 26 × 26 pixels in size and worked as RF for the feature detectors (units) in the V1 layer.

2. The V1 hidden layer consisted of $3 \times 3$ groups and each of these groups contained 8 x 8 units. A single group received input only from an area covering $26 \times 26$ pixels in 
the input layer (from corresponding RF). The RFs were also allowed to overlap at the edges by six pixels in order to maintain continuity of the images during processing. Together all the nine groups in the V1 layer extracted basic features in various parts of the input image in parallel.

3. The V2 hidden layer contained $2 \times 2$ groups of $8 \times 8$ units in size. Each of these groups received input from four groups in the V1 layer directly and from an area covering $46 \times 46$ pixels in the input indirectly.

4. The V3 hidden layer was $9 \times 9$ units in size and received input from all four groups in V2 layer directly and from the whole input layer indirectly.

5. The direction layer was represented by an $8 \times 1$ vector, where each unit indicated a direction ranging from $0^{\circ}$ to $315^{\circ}$ in $45^{\circ}$ increments. TC's movement towards $0^{\circ}$ and $360^{\circ}$ was represented by the left most unit and the right most unit represented a movement direction of $315^{\circ}$.

\subsubsection{Training and testing of the network}

Input-output pairs used to train the network were stored in a data-table as rows. Each row contained a $66 \times 66$ (for the input image) and an $8 \times 1$ (for the output movement directions) matrices to hold a single input-output pair. The network was trained in epochs using $95 \%$ of the 1200 generated images and the network completed 60 epochs during a batch (training run). Training of the network was stopped before the $60^{\text {th }}$ epoch, when the network produced zero error two times in a row. To be sure that the network's training performance was not influenced by the random initial weights, the network was trained for 10 runs.

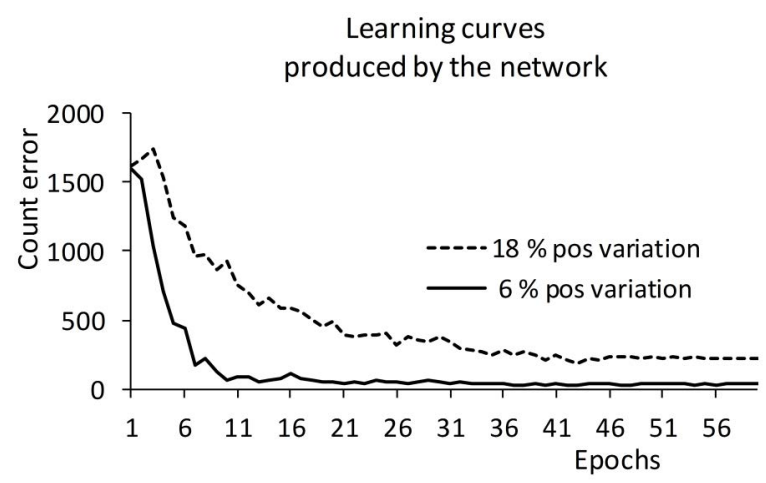

Figure 2-9. Training performance of the network obtained during the exploratory study.

Training error (number of images the network made a wrong forecast for) produced by the network during the 10 batches was averaged to produce the final training performance. Training error decreased rapidly within 10-15 epochs and stabilized at around 20 for rest of the epochs. This result was produced for the images, where the position of the TC center was varied by a maximum of $6 \%$ of the total image size but the training performance decreased, when inputs with greater position variation (18\%) were used (Figure 2-9).

Approximately 5\% of the 1200 generated images were presented to the network for the first time during testing. After training, the network was able to correctly predict the 
movement directions for $99 \%$ of the test images, when the position of the cyclone's center varied by $6 \%$ of the image size (Figure $2-10$ ). With a greater variation in the cyclone center's position within the image frame (18\% of the image size), prediction accuracy dropped to $84 \%$ (Figure $2-10$ ). For the test images that contained $18 \%$ positional variation, the wrong predicted directions were mostly opposite $\left(180^{\circ}\right.$ off $)$ or close $\left(45^{\circ}\right.$ off) to the correct direction. For the remaining images the predicted directions were perpendicular $\left(90^{\circ}\right.$ or $135^{\circ}$ ) to the correct direction (Figure 2-10).

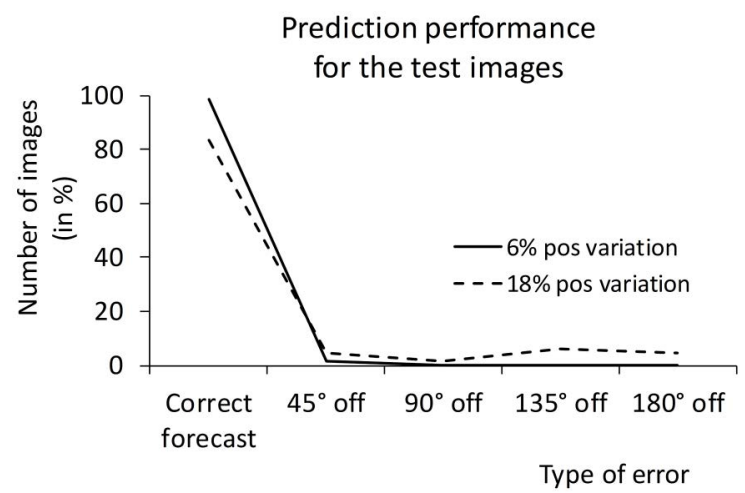

Figure 2-10. Test performance of the network obtained during the exploratory study. 


\section{Chapter 3}

\section{Methodological considerations}

Scientific research is based on some basic idea and has fixed goals to achieve. Upon this basis, a practical scheme is constructed to achieve the goals. An effective and relevant method is followed, which provides appropriate guidance to reach the research goals. In this way, methods play a key role in scientific research. However, is the method powerful enough to specify criteria to differentiate science from pseudoscience as well as can the method provide guidance for the progress of knowledge in science?

Karl Popper's falsificationism (Popper, 1959), Thomas Kuhn's structure of scientific revolutions (Kuhn, 1970), and Imre Lakatos' effort to harmonize the disagreement between Popper's and Kuhn's views and to develop a methodology for scientific research (Lakatos, 1978) are great contributions to the methodology of science. These methodologies provide guidelines for conducting research in a particular scientific field.

The basic idea of this thesis has been to use biologically based ANNs for producing accurate TC track and intensity forecasts, which could improve the efficiency of the current cyclone early warning system in countries, such as Bangladesh. This research implements the idea by identifying problems of the current cyclone early warning system and by considering the possibility of using biologically based ANNs for producing good accuracy TC track and intensity forecasts. Hierarchical neural networks inspired by the human visual system have been successfully used to achieve position and size invariance in processing visual images (O'Reilly, 1998; O’Reilly, Wyatte, Herd, Mingus, \& Jilk, 2013). Assuming that $2 \mathrm{D}$ information is of key importance to forecasting meteorological phenomena, these networks are expected to produce good accuracy TC track and intensity forecasts on the basis of TC images recorded by multiple satellites.

In order to identify problems associated with the current cyclone early warning system in Bangladesh and to evaluate the possibility of using biologically based ANNs for accurate TC forecasting, this thesis integrates knowledge from three different fields: (a) biologically based ANN simulation, (b) TC meteorology, and (c) natural disaster management (Figure 3-1). Biologically based ANN simulation deals with, construction of valid networks, network performance optimization, and training and testing the network systematically for producing TC forecasts. TC meteorology addresses how TCs are formed, which factors govern their motion and intensity and how TCs are forecasted. Eliciting providers' and receivers' views concern the process of collecting meteorologists' and residents' views on the current cyclone early warning system in Bangladesh. For managing and analyzing information from the three different fields, suitable method was also used. At this point two important questions could be: (a) is this research conducted with scientific rigor? and (b) can the method produce any results that have not been identified by previous studies? These two questions appear to be associated with the scientific quality as well as scientific 
contributions of this research, which could be difficult to answer without following a suitable research methodology.

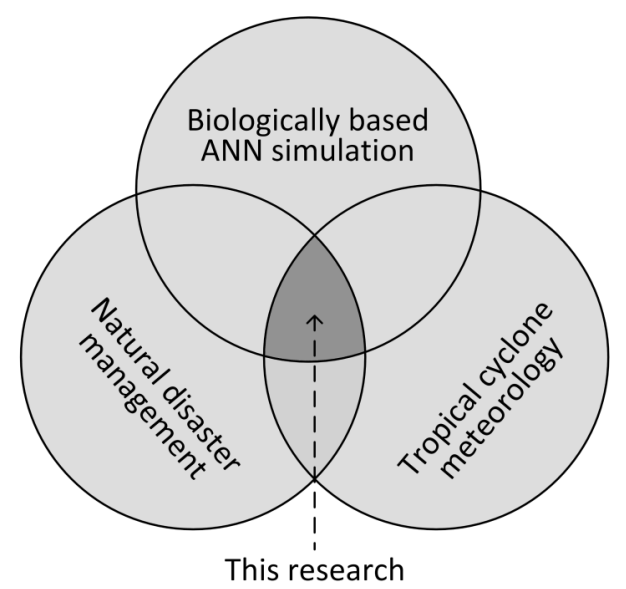

Figure 3-1. Biologically based ANN simulation, eliciting the views of meteorologists and residents, and tropical cyclone meteorology constitute the knowledge base of this research.

Karl Popper (Popper, 1959), Thomas Kuhn (Kuhn, 1970), and Imre Lakatos (Lakatos, 1978), all agree that scientific research is based on empirical observations. Qualitative and quantitative surveys conducted to evaluate cyclone early warning system use empirical observations to address research questions (Creswell, 2003; R. B. Johnson \& Onwuegbuzie, 2004). Also the data used for forecasting TC track and intensity are empirically based multi-source estimates of infrared (for cloud pattern and cloud top temperature), sea-level pressure, ocean heat content, wind speed, and wind direction. Therefore, all the data and observations used in this research are empirical or empirically based, which satisfies the very basic characteristics of a scientific research. However, only this criterion is not sufficient to differentiate between science and pseudoscience (Chalmers, 1999; Lakatos, 1978).

The application of biologically based ANNs is novel in the field of TC track and intensity forecasting (Kovordányi \& Roy, 2009; Roy \& Kovordányi, 2012, 2015). TC track and intensity forecasting techniques that are currently in operation at various meteorological offices have a long history of testing and modification (Kurihara, Bender, Tuleya, \& Ross, 1995; NCAR, 2014; NOAA, 2012; Roy \& Kovordányi, 2012, 2015). Also, forecasts produced by the operational techniques are on the basis of a large number of historical, persistence, and steering predictors (Hope \& Neumann, 1977; Jeffries et al., 1993; NCAR, 2014; Neumann, 1979; NOAA, 2012; Pike, 1985; C. S. Velden et al., 1998). As opposed to this, the technique developed in the thesis is a novel approach to TC forecasting, still under development, and uses only five types of images for forecasting TC track and intensity. Therefore, it does not seem reasonable to test its performance as if it were a full-scale operational technique, and try to subject it to falsification. Moreover, hypothesizing which factors influence network-generated outputs in order to prioritize one above another does not seem to agree with the potential existence of multiple equally important factors, which is considered to be a very important issue in ANN simulation as 
well as in TC forecasting. As Popper's methodology pushes theories and hypotheses towards comparison and seems to not support co-existence, Popper's methodology is not followed in the methodological structure of this research.

According to Kuhn, facts have to be considered relative to a paradigm, which supports the co-existence of different equally important theories and hypotheses within a paradigm. Some degree of relativism can be consistent with caring about facts. For example, a biologically based network using context layers and the same network without context layers (O'Reilly \& Munakata, 2000) might have different efficiency in predicting TC track and intensity. As these two networks handle input images in different ways, outputs from them could be a little different. However, the extreme relativism as proposed by Kuhn seems not appropriate for this research. If forecasts produced by the two networks appear to be very different and both are assumed to be equally correct, the whole purpose of TC track and intensity forecasting would be ruined. Therefore, the methodology of this research does not follow the structure of scientific revolutions proposed by Kuhn.

Lakatos' idea of scientific research programs is different from Popper's series of theories and from Kuhn's discontinuous open-ended scientific research schemes. The methodology of scientific research programs seems to provide more space within a paradigm in which novel research can develop. Moreover, Lakatos' notion of research programs appears to be more consistent with this research compared to Popper's and Kuhn's concept of theories. Therefore, Lakatos' methodology of scientific research programs is followed to constitute the methodological base of this research.

\subsection{Central and peripheral parts of this research}

This research consists of five interrelated studies (see section 1.3). The underlying theme of all these studies was that good accuracy TC track and intensity forecasts are essential for effective early warning and that biologically based ANN can satisfy that demand, where the core assumption has been that hierarchical ANNs inspired by the human visual system are efficient in processing spatially arranged $2 D$ data, and therefore should be efficient in TC track and intensity forecasting. This hypothesis constitutes the central part of this research (Figure 3-2). According to Lakatos' methodology, it is the hard core of this research. The central part allows all the studies to take place in this research and its existence is not dependent on the existence of any of the individual studies, which satisfies the methodological rule of negative heuristics proposed by Lakatos. The training and generalization ability of the biologically based ANNs used in this research is supported by a number of factors (Figure 3-2):

1. Hierarchical multi-layer architecture and combined learning assist feature extraction and recognition on the basis of $2 \mathrm{D}$ images.

2. Multi-source data integration is used, which is essential for producing good accuracy forecast.

3. Using greater number of images for training increases the probability of accurate forecasts during testing.

The more well-known machine learning approaches to TC track and intensity forecasting use vector representation of 2D satellite images (Feng \& Liu, 2004; Lee \& Liu, 2000), or one-dimensional tabular data (Jin et al., 2008) as inputs. In contrast, a hierarchical multilayer architecture and the use of simulated RFs (receptive fields) allow the networks to use 
2D satellite images as inputs. These capabilities allow the networks to use all the information contained in 2D input images without any loss for TC track and intensity forecasting.

A number of external and internal factors can affect TC track and intensity at any stage of its lifecycle (see section 2.2). As influences of these factors seem difficult to observe using a particular type of data, a forecasting technique's ability to integrate data from multiple sources is crucial for TC forecasting. A TC's recent past track and intensity can provide valuable information about its future track and intensity, so the ability to use this information is expected to have positive effects on TC track and intensity forecasting accuracy.

The network used in this research is capable of representing similarity relationships (O'Reilly, 1998); therefore, small variations between train and test datasets can be handled efficiently by the network. However, for handling large variations, both recent past information of the current TC and images of additional historical TCs need to be used for training. As the network is open to the number of images used for training and can use the prior time step's activation for task learning, it is expected to handle situations where variations in the training and test datasets are large.

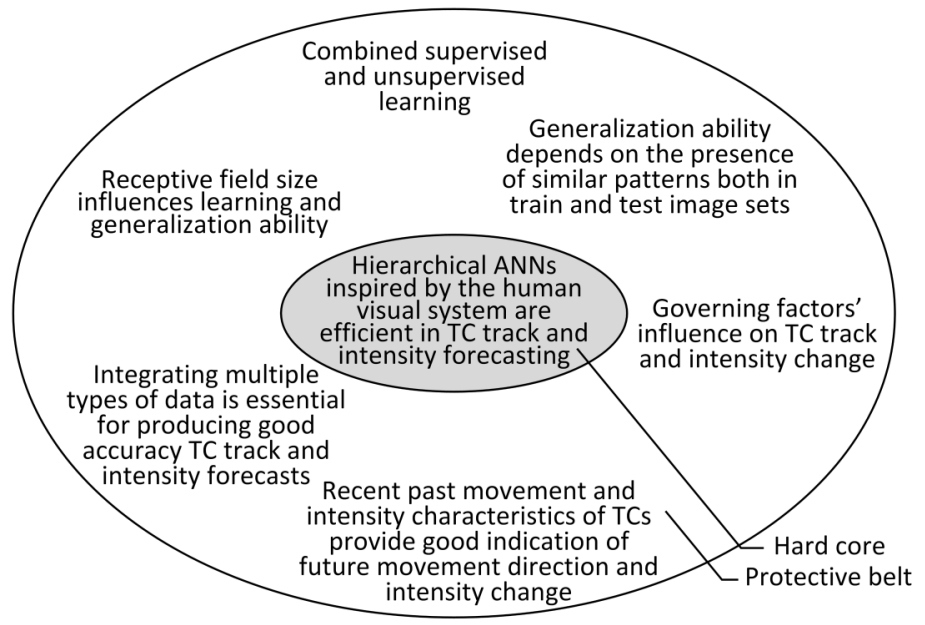

Figure 3-2. The hard core and the protective belt of this research.

In addition, activation-based receptive field analysis can be performed to reveal the underlying relationships between the patterns in the five types of input images and produced outputs. This seems to provide valuable information about, how individual factors effect TC track and intensity development. All the issues described in the previous two paragraphs imply that biologically based ANNs have the potential of producing good accuracy TC track and intensity forecasts as well as show directions for improving the quality of forecasts. Therefore, these issues constitute the protective belt of this research and satisfy the methodological rule of positive heuristics according to Lakatos' methodology of scientific research programs (Figure 3-2). 


\subsection{Technological paradigm of this research}

This research aims at developing a TC track and intensity forecasting technique using biologically based ANNs. The developed technique is expected to contribute to an improved cyclone early warning system in Bangladesh as well as in other cyclone-prone countries by producing good-accuracy TC track and intensity forecasts. At this point one important question could be, is this research consistent with any technological paradigm?

In a Technological Paradigm (TP), technologists set novel problems internally, whereas norms or standards for selecting techniques to solve those problems are set by external factors (Feibleman, 1966; Illich, 1974, 1978; Nordin, n.d.; Rapp, 1974). A TP gradually grows over time centering a technique and this growth takes place in the form of, number of novel practical problems solved by the central technique or by the auxiliary techniques within the paradigm.

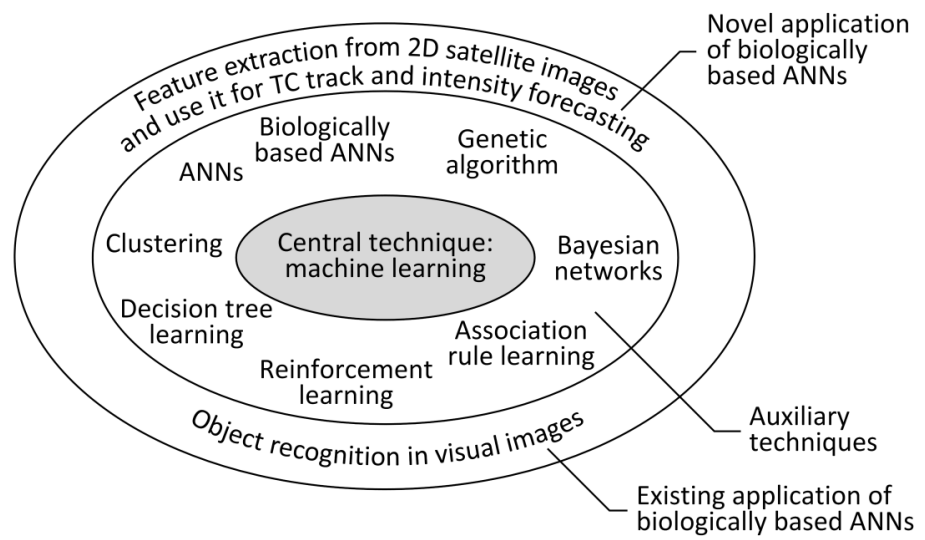

Figure 3-3. Machine learning paradigm and examples of auxiliary techniques.

Biologically based ANNs is an auxiliary technique in machine learning paradigm. This research targets to use this technique to solve a novel problem: TC track and intensity forecasting.

Goal of this research and nature of studies conducted to fulfill the goal make this research seem consistent with machine learning paradigm. Biologically based ANNs (a type of machine learning) are well-known for visual object recognition (O'Reilly, 1996; O'Reilly \& Munakata, 2000; Wyatte, Curran, \& O'Reilly, 2012) and this research aims at using it for forecasting TC track and intensity. Thus, an existing and well-known technique (biologically based ANN) is used to deal with a novel problem (TC track and intensity forecasting). However, biologically based ANN is not the only technique that follows the basic rules and methods of machine learning; several other techniques have also been developed under the guidance of the basic rules and methods of machine learning (Figure 3-3). Therefore, machine learning can be regarded as a paradigm, where basic machine learning rules and methods constitute the center of this paradigm and biologically based ANNs is one of the several auxiliary techniques within this paradigm (Figure 3-3). 


\section{Chapter 4}

\section{Method}

Informed product or system development is not new in design and usability research. In general, informed system development involves two interconnected processes: eliciting users for their perception of the system, and developing the system on the basis of those findings (Wright \& McCarthy, 2009). Accordingly, two processes are involved in TC track and intensity forecasting technique development in this thesis: (a) eliciting providers' and receivers' views and (b) TC track and intensity forecasting technique development (Figure 4-1). The two processes are described in the list below:

1. Eliciting providers' and receivers'views on the current cyclone early warning system in Bangladesh. The importance of reliable and informative warnings for eliciting expected response to evacuation orders has already been identified in previous studies (Akhand, 2003; C. E. Haque, 1995; U. Haque et al., 2012; B. K. Paul, 2012; B. K. Paul \& Dutt, 2010; B. K. Paul et al., 2010). However, why the warning messages are inaccurate and uninformative, as well as what can be done to improve the quality of the warnings have up till now remained unanswered. This research tries to answer these questions by eliciting the views both of the meteorologists at BMD (providers of the warnings) and of the residents in the coastal areas (receivers of the warnings).

2. Developing a TC track and intensity forecasting technique to address the technical issues brought to light in the previous step. As biologically based ANNs are efficient in visual image processing, they are expected to produce good accuracy forecasts when trained and tested on the basis of 2D TC images obtained from multiple sources. In addition, this technical approach can be limited to the use of freely available images. With these characteristics, it might be possible to disseminate reliable and informative warnings, when the developed technique would be put into operation in the future.

To elicit the providers' and receivers' views, and to develop a new TC track and intensity forecasting technique, five consecutive studies were conducted in this research. The studies together created a way to reach the stated objective of this thesis (Figure 4-1):

The first study identified the problems associated with TC forecasting and warning at BMD, as well as with the residents' response to evacuation orders in the coastal areas of Bangladesh. The effectiveness of a TC early warning system depends on both technical issues such as accurate forecasting and timely dissemination of informative warnings, as well as on residents' response to evacuation orders (Parker, 1999; WMO, 1989). Therefore, to cover-up the technical and residents' response related issues, both meteorologists' 
(providers of the warnings) and residents' (receivers of the warnings) views were elicited in the first study conducted in this research.

In the second study, TC track forecasting techniques were reviewed to find the technique that would be most suitable for addressing the issues identified in the first study. Assessing TC track forecasting techniques on the basis of: (a) predictor use, (b) prediction accuracy, (c) equation system use, and (d) computational resource requirements were the main concern of this study. Central parameters of the networks used for TC track and intensity forecasting were systematically tested in the third and the fourth study (see section 4.3.2).

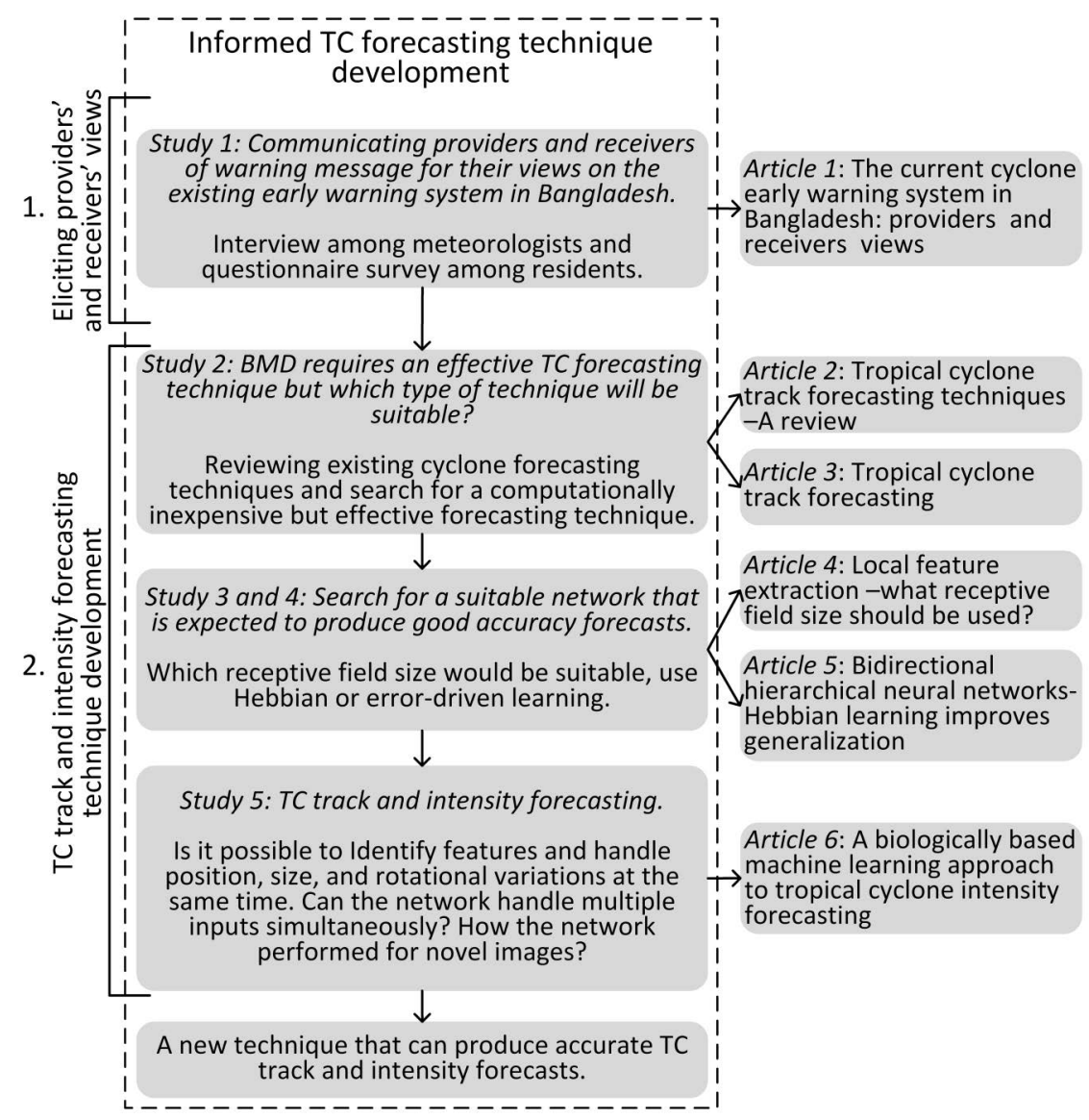

Figure 4-1. A simple flowchart of informed TC track and intensity forecasting technique development and contribution of the five studies in the process of TC track and intensity forecasting technique development.

In the fifth study, four biologically based ANNs were trained and tested using infrared, ocean heat content, sea-level pressure, wind direction, and wind speed images of nine TCs formed in the Atlantic basin during the years 2008 and 2009. Two of the four networks were used to forecast TC intensity for the next 6-, 12-, and 24-hours. The remaining two networks produced combined TC track and intensity forecasts only for the next 12 hours. 


\subsection{Study area}

Any country or region vulnerable to TCs could have been selected as the study area for this research. However, to successfully identify socio-economic and technical issues affecting cyclone early warning system, a number of criteria were considered for study area selection:

1. A large number of people live in areas where risk of being affected by TCs is high.

2. Both property damage and human casualties caused by TCs are high.

3. Geographical knowledge of the region, and ease of communication with residents living in the chosen cyclone prone areas (knowledge of language).

The whole coastal region of Bangladesh is considered to be cyclone prone (Islam et al., 2004). Of the nineteen coastal districts (MWR (Ministry of Water Resources), 2005), seven are considered as high cyclone-risk area and another seven are considered as moderate cyclone-risk area (M. A. Khan, Bhuyan, \& Rahman, 2010). Despite the risk of being affected by TCs and associated storm surges, currently around thirty and a half million people are living in these fourteen high and moderate cyclone-risk districts (Figure 4-2) (BBS (Bangladesh Bureau of Statistics), 2012). As the work presented in this thesis involved interviews and questionnaire survey, ease of verbal communication (knowledge of the language) and appropriate geographical knowledge about the study area were considered important.

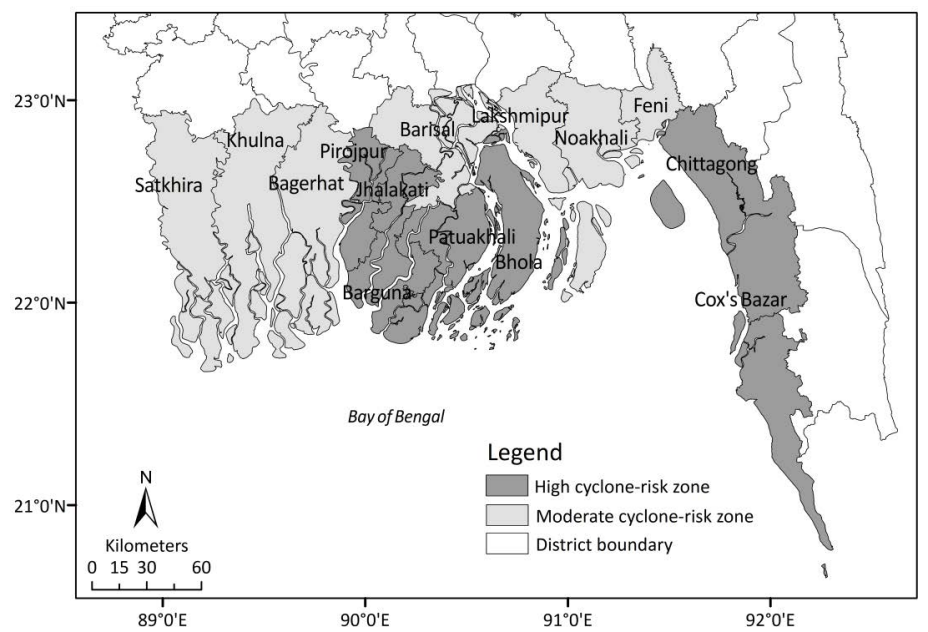

Figure 4-2. Distribution of cyclone risk-zones in the coastal area of Bangladesh. Figure prepared based on the cyclone risk zone classification proposed by (Khan et al., 2010).

TCs have caused a great number of human casualties in Bangladesh historically (CRED, 2013; C. E. Haque, 1997; Yang \& Wang, 2005). In addition, the author knows the local language "bengali" and culture, and has proper geographical knowledge of the coastal regions of Bangladesh. Therefore, Bangladesh was considered most suitable for this study. Other countries exposed to TCs, such as India, Myanmar, China, or Japan could have been selected for the study but these countries do not meet all the criteria listed above for study area selection. 
Residents living in different disaster-risk zones might have different views on disaster early warning system (Sahni \& Ariyabandu, 2004; Sahni \& Dhameja, 2004). Therefore, to elicit the views of residents living in both moderate and high cyclone-risk areas, the moderate cyclone-risk district Bagerhat and the high cyclone-risk district Patuakhali were selected (M. A. Khan et al., 2010) for the study. These districts are also among the three districts (Bagerhat, Patuakhali, and Barguna) where the damage caused by TC Sidr was the greatest (roughly between 193.66 and 245.30 million USD as of October 2014) (GoB (Government of Bangladesh), 2008).

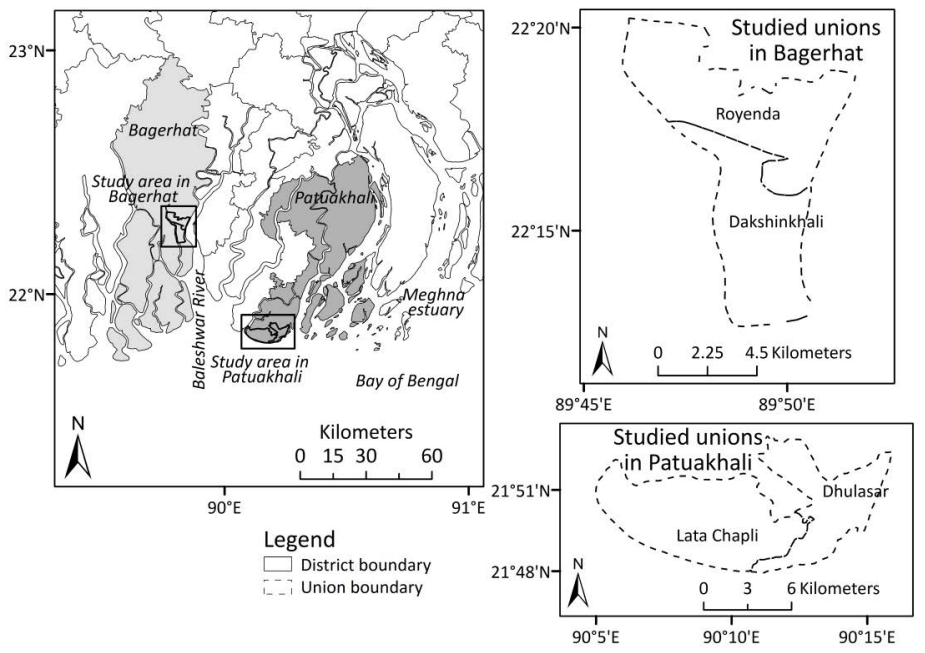

Figure 4-3. Locations of the studied unions in the coastal region of Bangladesh.

Patuakhali and Bagerhat, comprise 72 and 75 unions (small administrative units), respectively. A simple random sampling method was used to choose two unions from Bagerhat, namely Royenda and Dakshinkhali, and two unions from Patuakhali, namely Dhulasar and Lata Chapli, for the data collection (Figure 4-3). These four unions in the coastal region of Bangladesh have constituted the study area for conducting the questionnaire survey in this research (see section 4.2.2).

\subsection{Eliciting providers' and receivers' views}

Cyclone early warning constitutes a socio-technical system, which functions through two phases: (a) TC forecasting and warning message dissemination, and (b) residents' response to the disseminated warnings. To be able to assess the effectiveness of the two phases, the views of both the meteorologists (providers of the warnings) and the residents in the coastal area (receivers of the warnings) were elicited. In-depth interviews are considered to be suitable for collecting detailed information from relatively few respondents in the case of evaluating a socio-technical system (Berg, Wetherell, \& Houtkoop-Steenstra, 2003; Boyce \& Neale, 2006; Guion, Diehl, \& McDonald, 2011; Legard, Keegan, \& Ward, 2003; Parker, 1999). Questionnaire surveys in contrast, are commonly used for: (a) classifying a population on desired bases, such as age, occupation, and housing condition; (b) assessing people's opinion regarding a particular issue or issues; and (c) collecting information related to people's behavior (Bradburn, Sudman, \& Wansink, 2004; Guha-Sapir \& Lechat, 
1986; Kurita, Nakamura, Kodama, \& Colombage, 2006; Mallick et al., 2009; Shaw, Kobayashi, \& Kobayashi, 2004). Therefore, a questionnaire survey was conducted among the residents in the coastal area. A research team of three members, including the author of this thesis, conducted an interview among the meteorologists and a questionnaire survey among the residents in the coastal area.

\subsubsection{Interview among the meteorologists}

At BMD, an individual in-depth interview was conducted among three meteorologists. The meteorologists were between 41 and 53 years of age, and had all been working at BMD for more than 10 years. Two of the three meteorologists had a background in physics and one had a background in mathematics. The Storm Warning Center (SWC) at BMD is responsible for monitoring, forecasting, and disseminating warnings for TCs in Bangladesh. Therefore, meteorologists only from this unit were selected for the interview. As none of the SWC meteorologists had a meteorological or other atmosphere-related background, we only interviewed those meteorologists who had long work experience (at least ten years) in TC forecasting. This selection criterion limited the number of participants to three at BMD.

The three respondents were interviewed individually. A range of open-ended and structured questions was used for the interview (Appendix D). The questions were designed to cover all the technical aspects of TC forecasting that could influence the quality of the disseminated warnings (Table 4-1). All the questions were asked orally and the meteorologists answered the questions also orally. The meteorologists' opinion on the open-ended questions and their answers for the structured questions were noted. A short follow-up interview was also conducted among the same three meteorologists, where they were asked to draw a flowchart describing the processes of TC forecasting at BMD.

A method based on deductive thematic analysis (Braun \& Clarke, 2006; Fereday \& Muir-Cochrane, 2008) was used to analyze the results obtained through the interview. As the aim of the interview with the meteorologists was to gather information on various technical aspects of TC forecasting and warning (Table 4-1), the features that could be used for describing these aspects were coded in the answers received from the meteorologists. Six potential themes were identified out of the data using a semantic analysis approach (Boyatzis, 1998). The codes, as well as the data relevant to each potential theme were brought together at this stage (Table 4-2). To avoid unnecessary division/union of the themes, the identified themes were reviewed on the basis of their internal homogeneity and external heterogeneity (Patton, 1990).

Table 4-1. Focus of the interview and the technical aspects covered by the questions.

\begin{tabular}{lll}
\hline \multicolumn{1}{c}{ Focus } & Technical aspects covered by the questions \\
\hline TC forecasting & - Meteorological data collection and use \\
& - Methods of atmospheric data analysis \\
& - Techniques used for TC track and intensity \\
& prediction \\
& - Forecast accuracy and forecast length \\
& - Cooperative activities across agencies \\
\hline Warning dissemination & - Processes of warning message formulation \\
& - Media of warning dissemination \\
\hline
\end{tabular}


Limitations and future development plans
- Limitations of the existing TC forecasting and warning system

- Meteorologists' views on future development plans

Table 4-2. Potential themes and relevant primary codes.

\begin{tabular}{ll}
\hline \multicolumn{1}{c}{ Potential themes } & \multicolumn{1}{c}{ Primary codes } \\
\hline Data collection & $\begin{array}{l}\text { Ground-based weather stations, ocean buoy, radar, } \\
\text { weather balloons, satellites, atmospheric reanalysis data }\end{array}$ \\
\hline $\begin{array}{l}\text { TC forecasting and } \\
\text { warning formulation }\end{array}$ & $\begin{array}{l}\text { Model run time, prediction accuracy, computational } \\
\text { resource requirement, forecast length, wind data } \\
\text { plotting, steering level, historical analogues, persistence }\end{array}$ \\
\hline International cooperation & $\begin{array}{l}\text { Technical support, RSMC, WMO, Meteorological } \\
\text { offices }\end{array}$ \\
\hline Limitations & $\begin{array}{l}\text { Data scarcity over the BoB, expertise of the } \\
\text { meteorologists, computational resources, forecast } \\
\text { verification, quality of the produced forecasts }\end{array}$ \\
\hline $\begin{array}{l}\text { Warning message } \\
\text { dissemination }\end{array}$ & $\begin{array}{l}\text { Warning formulation guidelines, reliability of the } \\
\text { disseminated warnings, national disaster management } \\
\text { organizations, national disaster coordination cell, } \\
\text { message content, RSMC, WMO }\end{array}$ \\
\hline Future development plans & $\begin{array}{l}\text { Using numerical models, cooperation, developing } \\
\text { infrastructures, using new data for forecast, deploying } \\
\text { new signaling system. }\end{array}$ \\
\hline
\end{tabular}

The six potential themes were combined and/or broken down into three themes that were most consistent with the main focus of the interview among the meteorologists (Table 4-1). Finally, to avoid duplication, the codes were reviewed and were assigned to the three themes on the basis of their relevance (Table 4-3). Answers received from the meteorologists during the survey are provided under these three themes in section 5.1. A TC forecasting flowchart was also prepared on the basis of the flowcharts drawn by the meteorologists and presented in the results section.

Table 4-3. The three final themes and relevant codes.

\begin{tabular}{ll}
\hline \multicolumn{1}{c}{ Final themes } & \multicolumn{1}{c}{ Refined codes } \\
\hline TC forecasting & $\begin{array}{l}\text { Data collection, prediction accuracy and length, data } \\
\text { analysis. }\end{array}$ \\
\hline $\begin{array}{l}\text { Warning message } \\
\text { formulation and } \\
\text { dissemination }\end{array}$ & $\begin{array}{l}\text { Self-produced forecasts, eleven point signaling system } \\
\text { for the maritime porta, four point signaling system for } \\
\text { river ports, RSMC forecasts, national disaster } \\
\text { management organizations, safety measures for } \\
\text { residents. }\end{array}$ \\
\hline $\begin{array}{l}\text { Limitations and future } \\
\text { development plans }\end{array}$ & $\begin{array}{l}\text { Computational resource requirements, deploying new } \\
\text { techniques for forecasting TCs, deploying new TC } \\
\text { signaling system, meteorologists lack necessary skills, } \\
\text { cooperation for enhancing skills, using new data for } \\
\text { better coverage over the BoB. }\end{array}$ \\
\hline
\end{tabular}




\subsubsection{Questionnaire survey among the residents in the coastal areas}

The questionnaire used to elicit respondents' views on the current cyclone early warning system consisted of two parts (Appendix E). The first part contained questions for collecting socio-economic information of the respondents and all the questions used in this part were structured questions. The second part of the questionnaire was devoted to elicited respondents' views on TC warnings and only multiple-choice questions were used in this part.

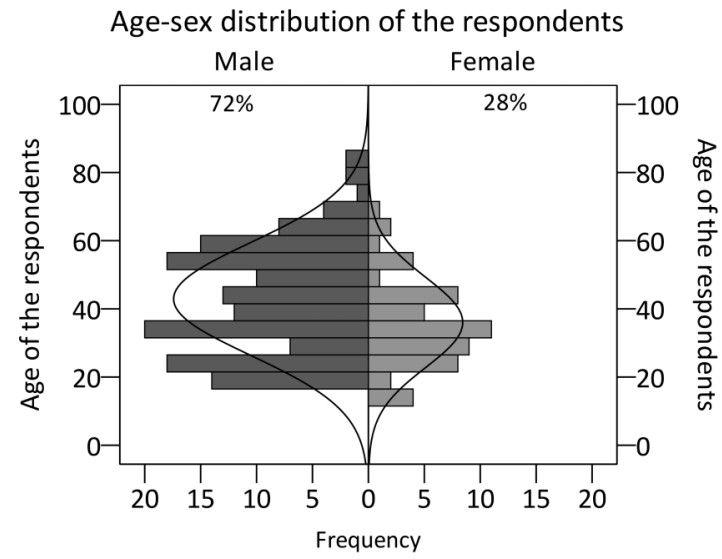

Figure 4-4. Age and sex distribution of the surveyed respondents.

The actions taken by the residents in cyclone emergencies might be influenced by the forecasted intensity level of the approaching TC (Riad, Norris, \& Ruback, 1999). Therefore, to elicit a generalized warning response pattern of the residents in the coastal area, two of the recent TCs, namely the severe Sidr and the less severe Mahasen were selected as focus for the data collection in this research.
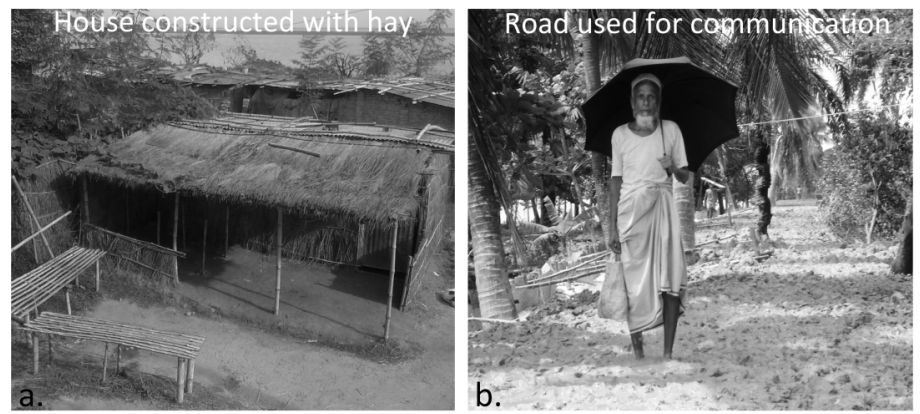

Figure 4-5. Housing condition and accessibility to road transportation. a. Houses are mostly katcha and $\mathbf{b}$. road transportation is mostly poor. Photographs taken by the author during the survey.

The primary sampling units were individual households. Lists of all the households in the four selected unions were collected from the respective union parishads (union administrative offices) prior to administering the survey. Two hundred households were 
selected from the four unions using simple random sampling. The data were collected from adult persons (who were available during the survey) in each household. The respondents were between 18 and 84 years of age. Around $70 \%$ of them were male and $30 \%$ were female (Figure 4-4).

The respondents were involved in farming (24\%), fishing (12\%), service $(10 \%)$, business $(9 \%)$, and in multiple occupations (31\%) like farming, fishing, and construction work (e.g. road and dam) for their livelihood, and the remaining 14\% were dependent on the main earning member of the family. Around $75 \%$ of the respondents earned between 2000 and 7000 taka (26 and 115.74 USD as of December 2014) per month. The surveyed households consisted mostly ( $78 \%$ of the 200 households) of 3 to 6 members. Most of the surveyed households ( $86 \%$ ) were katcha (floor of mud, wall of wood/tin/hay, roof of tin/hay) and the respondents mostly (76\%) had mud-built roads surrounding their home (Figure 4-5).

The questions in the second part of the questionnaire (Appendix E) were designed to elicit participants' views of the disseminated warning as well as to elicit their suggestions for improving warnings in the future (Table 4-4).

Table 4-4. Focus of the questionnaire survey and residents' reaction related aspects covered by the questions used for the survey.

\begin{tabular}{lll}
\hline \multicolumn{1}{c}{ Focus } & \multicolumn{1}{c}{ Aspects covered by the questions } \\
\hline Reception and interpretation & - Media of warning message dissemination \\
of the warning message & - Media preferred by the respondents \\
& - Respondents' ability to interpret \\
& - $\begin{array}{l}\text { Influence of socio-economic status on the } \\
\text { reception of warning messages by the }\end{array}$ \\
& - respondents \\
\hline Response to warnings & - Rvacuation pattern among the respondents \\
& - Influence of socio-economic status on the \\
& & respondents' evacuation behavior \\
\hline Level of satisfaction with the & - Respondents' satisfaction level \\
disseminated warnings and & - Reasons for satisfaction \\
suggestions for improvement & - Suggestions for improving warnings in the \\
& & future \\
\hline
\end{tabular}

The respondents were allowed to make multiple answers for each multiple-choice question used in the second part of the survey. They were also permitted to express additional comments and views in free text at the end of each question. As pre-recorded warning messages were used to reveal respondents' ability to understand warning message content and as the respondents might have difficulties in understanding all the questions in the questionnaire, the survey team asked all the questions verbally and noted the responses themselves for each of the 200 respondents.

In the beginning of the survey, the respondents were asked to express their views on the media currently used for warning dissemination. Whether the respondents made an evacuation during the two selected TCs and reasons for their decision were asked at this point. Then, to get insight into the respondents' ability to interpret warning message 
contents, they were let to listen four pre-recorded warning messages (Appendix F), of which two messages were disseminated during TC Sidr and the other two messages were disseminated during Mahasen. After letting the respondents to listen, they were asked to describe the information contained in the messages and to highlight the differences between the two warning messages for the same TC. Finally, the respondents were asked to mention their satisfaction level with the disseminated warnings during the two studied TCs on a 5point scale (where points 1 and 5 indicate not satisfied and satisfied at a very high level, respectively). They were also asked for suggestions to improve TC warnings in the future. Several statistical techniques: multiple-response analysis, frequency distribution, binary logistic regression, factor analysis and cross tabulation were used to analyze the answers obtained in the second part of the questionnaire (Table 4-5).

Table 4-5. Statistical techniques uses to analyze the participants' response received in the second part of the questionnaire.

\begin{tabular}{|c|c|}
\hline Statistical techniques & Used to analyze \\
\hline $\begin{array}{l}\text { Multiple-response analysis (Edwards } \\
\text { \& Allenby, 2003; Santos, 2000) }\end{array}$ & $\begin{array}{l}\text { - Media through which the residents } \\
\text { received warning messages during the } \\
\text { two TCs } \\
\text { - } \quad \text { Through which media the residents } \\
\text { would like to receive warning messages } \\
\text { - Reasons for preferring those media } \\
\text { - Reasons for selecting the warning } \\
\text { satisfaction level } \\
\text { - Suggestions for improving warning } \\
\text { messages in the future }\end{array}$ \\
\hline Binary logistic regression & $\begin{array}{l}\text { - The media's efficiency for warning } \\
\text { message dissemination }\end{array}$ \\
\hline Frequency distribution & $\begin{array}{l}\text { - Accurate interpretation of the warning } \\
\text { message content }\end{array}$ \\
\hline $\begin{array}{l}\text { Factor analysis using Principal } \\
\text { Component Analysis (PCA) }\end{array}$ & - Reasons for non-evacuation \\
\hline Cross product calculation & $\begin{array}{l}\text { Correlation between two or more } \\
\text { variables, such as socio-economic } \\
\text { status, reception of warning and } \\
\text { response to warning }\end{array}$ \\
\hline
\end{tabular}

\subsection{TC track and intensity forecasting technique development}

In the second study, TC track forecasting techniques were reviewed to understand:

1. Predictor use, which types of data are used by various TC forecasting techniques.

2. Equation system use, which equation systems are used for producing TC forecasts.

3. Computational resource requirements, computational resource required to run various forecasting techniques.

4. Prediction accuracy, accuracy of forecasts produced by various TC forecasting techniques. 
Though numerical TC track-forecasting techniques are the most advanced and the best performing among all the operational TC track-forecasting techniques (Rogers et al., 2006; Roy \& Kovordányi, 2012, 2015), numerical techniques are not capable of producing TC intensity forecasts with similar accuracy (Cangialosi \& Franklin, 2014; DeMaria et al., 2007; DeMaria, Mainelli, Shay, Knaff, \& Kaplan, 2005). Numerical techniques are usually complex, require high-end computers to run, and are vulnerable to inaccurate initial values (Jeffries et al., 1993; Roy \& Kovordányi, 2012). These issues have motivated research into novel approaches for processing images to forecast TCs.

ANNs inspired by the human visual system are well-known for their ability to recognize objects in 2D visual images (Fukushima, 2013; Itti et al., 1998; LeCun et al., 2015; O'Reilly et al., 2013). TC track and intensity forecasting on the basis of the patterns detected in multi-source 2D images and object recognition in 2D visual images are typically similar tasks; therefore, biologically based ANNs were used to produce TC track and intensity forecasts in this research.

This research involves simulation of four biologically based ANNs. Of the four networks, two were simulated for TC intensity forecasting and two networks were simulated for combined TC track and intensity forecasting. Similar to other simulation model construction, a validation criterion was followed while constructing the four networks to make sure that the technique could be used to produce good accuracy forecast for its users after development. In the process of network construction, a three-step approach was followed to ensure the validity of the four networks (Law \& Kelton, 1991; Van Horn, 1971). The three steps are described in detail in the next three subsections. The datasets used for TC track and intensity forecasting, structure of the four networks, information processing in the four networks, training and testing procedures, and how feature detection works in the four networks are described in subsections 4.3.4 to 4.3.10.

\subsubsection{Efficiency of the simulation tool with respect to $2 \mathrm{D}$ image processing}

Selecting an effective tool for constructing and simulating the four networks constituted the first step of network validation. Three-layer feed forward networks have been previously used for TC track and intensity prediction (Ali et al., 2007; Jin et al., 2008; G. P. Johnson \& Lin, 1995; Lee \& Liu, 2000; Pickle, 1991). However, these networks are not capable of simultaneous feature extraction from 2D images and cannot take into account the spatial relationship of the extracted features relative to the TC vortex. Therefore, robust pattern recognition from multiple images regardless of random variations in position and size relative to the image frame is hard to achieve using standard three-layer feedforward networks.

Though biologically based ANNs have not yet been used for TC track and intensity forecasting, these networks are well-known for recognizing objects in visual images (Fukushima, 2013; LeCun et al., 2015; Li \& Itti, 2011; O'Reilly et al., 2013; Wyatte, Curran, et al., 2012). The problem of feature extraction from TC images and using it for TC track and intensity forecasting is similar to the problem of object recognition in visual images. Therefore, biologically based ANNs were used to produce TC track and intensity forecasts in this research.

The ANN simulation tool Leabra (Local, Error-driven and Associative, Biologically Realistic Algorithm), which is available as a tool in the open-source neural network simulator emergent was used to construct and simulate the networks in this research (Aisa, 
Mingus, \& O'Reilly, 2008; CCNLab, 2014; O’Reilly \& Munakata, 2000). Emergent has a long development history. This neural network simulator was first introduced as PDP++ already in 1986. After development, this simulator has been used, tested, and improved by a community of researchers, which establishes Leabra as a valid tool for the simulation.

\subsubsection{Testing assumptions}

Network-generated outputs could be sensitive to a wide range of preconditions, so different assumptions were handled carefully and systematically to reduce the network's sensitivity to variations in certain preconditions (Law \& Kelton, 1991). As assumptions are often unclear and difficult to track during the simulation, they can be difficult identify by those who are using the simulation results. For this reason, systematic assumptions handling is necessary (Eriksson, Morin, Ekberg, Jenvald, \& Timpka, 2009). Of the seven categories of assumptions suggested by Eriksson and coauthors (2009), model assumptions, data assumptions, parameter assumptions, and control assumptions were handled systematically during construction of the networks. The remaining three categories were found irrelevant during network construction, because:

1. Paradigm assumptions deal with underlying architectural properties and operative principles of a simulator. As these are given within the simulator used in the thesis, managing paradigm assumptions fell outside the scope of the present work.

2. Behavioral assumptions concern human behavior and its interference in a simulation. As only physical processes govern TC track and intensity, managing behavioral assumptions were not necessary.

3. Scenario assumptions refer to external context that might influence the simulation results. In this thesis, scenario could correspond to factors, such as cyclone formation basin. As this thesis does not compare the simulation performance for different basins, these assumptions were not managed.

\section{Model assumptions}

Model assumptions addressed which meteorological factors could be used for forecasting TC track and intensity. A number of factors govern TC track and intensity development (see section 2.2). Some of these factors are more important for determining the TC's future track and intensity than others (Sampson, Jeffries, \& Neumann, 1995; Sampson, Jeffries, Neumann, et al., 1995). For example, cumulonimbus cloud patterns visible in infrared satellite images, provide a very good indication of a TC's track in the near future (Lajoie, 1976; Lajoie \& Nicholls, 1974) as well as TC's future intensity change (Dvorak, 1972, 1975). Therefore, satellite-derived infrared images are considered to be an important predictor for forecasting TC track and intensity. In addition, ocean heat content (Wada \& Usui, 2007), sea-level pressure (Holliday \& Thompson, 1979; Knaff \& Zehr, 2007), wind direction and speed (Mueller et al., 2006; Sampson, Jeffries, Neumann, et al., 1995), and cloud-top temperature (Dvorak, 1984, 1995; Gentry et al., 1980) are among the influential factors governing TC track and intensity. Therefore, these five types of information (infrared image, ocean heat content, sea-level pressure, wind direction, and wind speed) were used for forecasting TC track and intensity in this research. 


\section{Data assumptions}

Data assumptions referred to issues associated with the availability and quality of the data used for TC track and intensity forecasting as well as issues related to the interpretation of the outputs in relation to this data. The images used in this research were recorded by multiple instruments. The spatial coverage, and spatial-, temporal-, and radiometricresolution of the TC images were also different. As these variations were related to the quality and availability of the data, outputs from the four networks could be sensitive to these variations. To reduce the networks' sensitivity to these variations, several data assumptions were handled:

1. Managing spatial coverage: five types of images with different spatial coverage were used for TC track and intensity prediction. All the original images were cropped into $14^{\circ}$ latitude $\times 14^{\circ}$ longitude squares centered on the TC eye, so that the TC vortex and the surrounding environment containing valuable information about TC track and intensity change could be captured in the same frame. This also allowed the four networks to be fed with different types of information covering the same area.

2. Managing spatial resolution: An ordinary PC, with $2.4 \mathrm{GHz}$ quad-core Intel CPU, using 3 gigabytes of RAM, was used for training and testing of the four networks. To match this low-end computational resource, low-resolution images were used for TC track and intensity forecasting. Spatial resolution of the five types of cropped images were downsized (upsized in the case of sea-level pressure images) to $60 \times 60$ pixels.

3. Managing temporal resolution: Temporal resolution of the five types of inputs were not the same. Though, infrared image, sea-level pressure, wind direction, and wind speed images are available four times a day, ocean heat content is available only once a day. The same ocean heat content image was used four times to match the temporal resolution of the other input images.

4. Managing radiometric resolution: Radiometric resolution of the images used for TC track and intensity forecasting was not the same. Using of these images could result in large variations in the four networks' activation pattern. To handle this problem, all the images were first converted into 8-bit grayscale, and then the pixel values of this grey scale images were compressed into the interval 0 and 1 . As the original pixel values were transformed linearly in two steps, the information contained in the original image was not lost.

5. Managing position variations: During training, if cloud and other patterns contained in the images appear at the same position in the input frame all the time, feature identification by the four networks could become sensitive to the features' position, which could influence learning of the networks. Therefore, each image was shifted in position by zero, one, or two steps along both $\mathrm{x}$ - and y-axes. In this way, eight shifted images were generated $(<\mathrm{x} 0, \mathrm{y} 1>,<\mathrm{x} 0, \mathrm{y} 2>$, and so on) from each image. The size of the shift was two pixels at each step, so the shifted image differed in position by a maximum of four pixels (ca. $7 \%$ of the image size) compared to the original image. As shape and size of cloud patterns are associated with TC intensity (Dvorak, 1972, 1975) as well as with TC motion (Lajoie \& Nicholls, 1974), the images were not rotated or zoomed, when preparing the datasets for training and testing the networks. 
6. Managing forecast length: In this research, TC intensity forecasts were produced for 6-, 12- and 24-hours ahead. Three different datasets were also generated for producing intensity forecasts for three different lengths. For the 6-hour forecast datasets, images recorded at 6-hour intervals were used as inputs and the maximum wind speeds observed at 6 -hour intervals were used as output intensity levels. Sixhour time difference between the input image and the output intensity level was also used for forecasting TC intensity levels 6-hour ahead on the basis of the current input presented to the networks. For the 12-hour forecast datasets, the time differences between two input images and between two maximum wind speed observations were 12 hours. In this case, the time difference between the input image and the output intensity level was set to 12 hours. The datasets for 24-hour forecast were also prepared in the same way. Combined TC track and intensity forecasts were produced only for 12-hours ahead. Images recorded at 12-hour intervals were used as inputs. Maximum wind speeds observed at 12-hour intervals constituted the intensity part of the output, whereas TC eye positions in two images recorded at 12-hour intervals and distance between these two eye positions together created the track part of the output. A 12-hour time difference between input image and output track and intensity levels was also used.

\section{Parameter assumptions}

Parameter assumptions referred to the values of different parameters used for the simulation. Emergent allows for highly customizable networks for simulating biologically based ANNs (O'Reilly, 1998; O'Reilly \& Munakata, 2000). However, for simulating the four networks, the default values were used for most of the parameters. The values were changed for the following parameters, to improve learning and generalization performance:

1. RF (receptive field) size

2. Threshold value for firing output activation or firing threshold

3. Amount of Hebbian and error driven learning, and

4. Activity level over the layers.

Size of the RFs of the units in the first hidden layer is important for task learning and generalization ability of a network (O'Reilly \& Munakata, 2000). The four networks were systematically tested using RFs of varying size on the basis of the TC images in Table 4-6 to get the optimal RF size for TC track and intensity forecasting in this research.

Table 4-6. RF size and availability of meaningful features in the RFs.

\begin{tabular}{cccc}
\hline $\begin{array}{c}\text { TC images used for } \\
\text { network performance } \\
\text { optimization }\end{array}$ & \multicolumn{3}{c}{ Receptive field size (pixels) } \\
\cline { 2 - 4 } & $26 \times 26$ & $20 \times 20$ & $16 \times 16$ \\
\hline & & & \\
\hline & & & \\
\hline
\end{tabular}




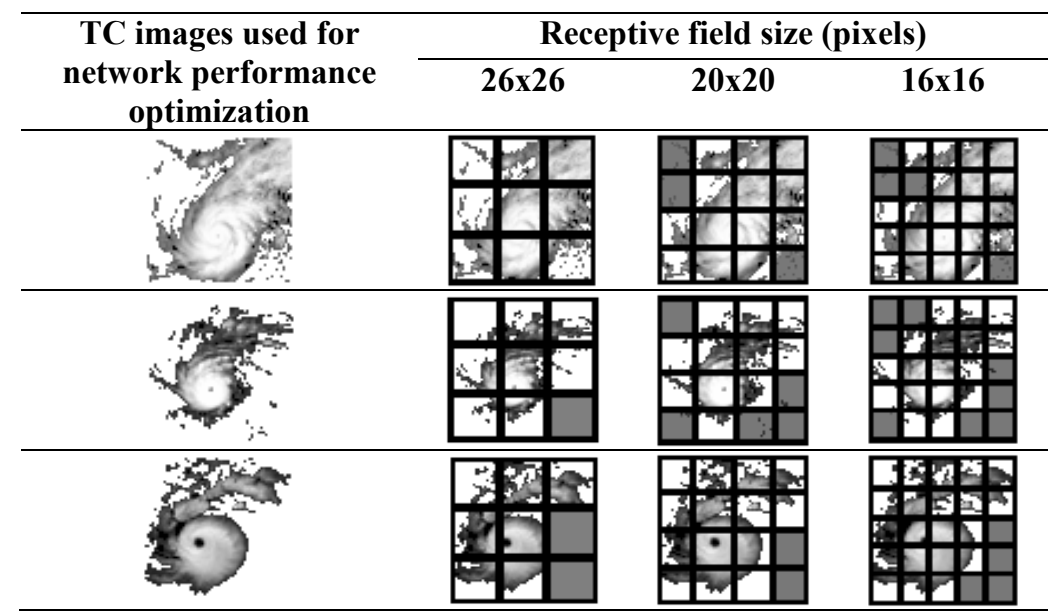

Note: RFs that contain a few/no meaningful features are marked using grey background.

Leabra uses a biologically derived activation function, to mimic the information processing dynamics in the human brain (O'Reilly \& Munakata, 2000). How strongly a unit should react to input can be expressed by the following biologically based sigmoid activation function (Equation 1):

$$
y_{j}=\frac{\gamma\left[V_{m}-\Theta\right]_{+}}{\gamma\left[V_{m}-\Theta\right]_{+}+1}, \quad[Z]_{+}=\left\{\begin{array}{l}
z \text { if } z \geq 0 \\
0 \text { if } z<0
\end{array}\right.
$$

\section{Equation 1}

Where,

$y_{j}=$ activation of receiving unit $j$

$\gamma=$ gain

$V_{m}=$ membrane potential

$\Theta=$ firing threshold

The output activation produced by each single unit can be changed by changing the value of $\Theta$. In emergent, the default value of $\Theta$ is 0.50 . However, for the four networks used for TC track and intensity forecasting, $\Theta=0.47$ was used. The pattern of bright pixels capable of producing strong input was not evenly distributed in all the images used for track and intensity forecasting in this research (Figure 4-6). For example, in the sea-level pressure images, pixel values were remarkably low, whereas in the wind speed images high-value pixels appeared in clustered form (Figure 4-6). The default value of $\Theta$ could not assist the units to produce activation outputs useful for the task; therefore lowering of the $\Theta$ value was necessary. With a lower $\Theta$ value, more units could produce activation outputs during training.

The proportion of Hebbian (unsupervised) and error driven (supervised) learning is another important issue for task learning and generalization. Hebbian learning enhances the generalization ability in recurrent hierarchical networks through imposing locally derived constraints on weight development (O'Reilly \& Munakata, 2000). Also, locally developed feature detectors facilitate the processes of feature extraction and image processing during the generalization phase. The four networks were systematically tested using different combinations of Hebbian and error driven learning to get the combination, which is most suitable for TC track and intensity forecasting. 
Leabra uses kWTA (k-Winners-Take-All) to achieve inhibitory competition among the units within a unit group and/or within a layer (O'Reilly \& Munakata, 2000; Wyatte, Herd, Mingus, \& O'Reilly, 2012). This inhibition allows exactly $k$ number of units to become activated above threshold. In this research, $25 \%$ of the units within each group/hidden layer were allowed to be active. To ensure that only a single unit became active in the output layers (representing a certain movement direction or intensity level), the value $k=1$ was specified directly.
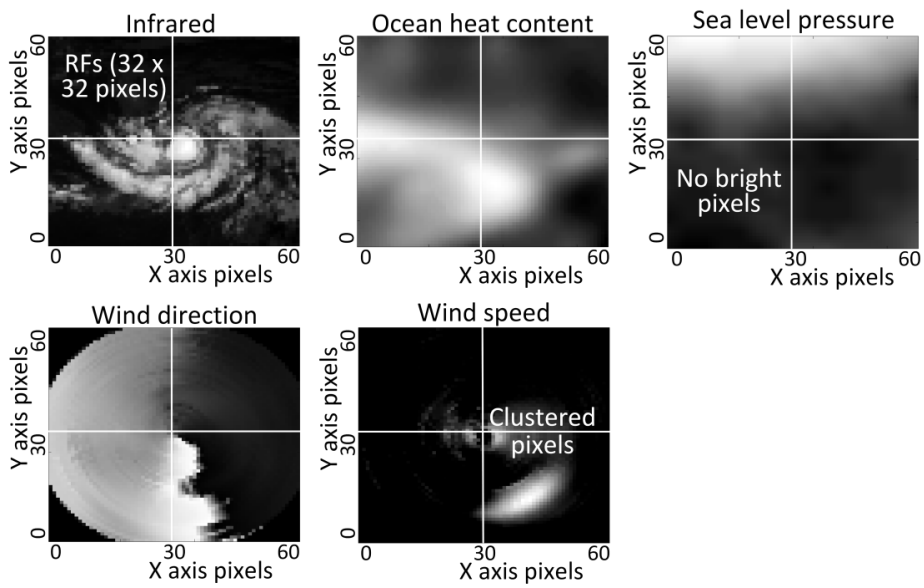

Figure 4-6. RFs and distribution pattern of high, medium, and low value pixels in the five types of images used for TC intensity forecasting.

\section{Control assumptions}

Control assumptions tackled the criteria for terminating or continuing the simulation runs. All the four networks were trained in epochs ${ }^{3}$. The networks were allowed to run a maximum of 50 epochs and each of the four networks completed 50 epochs during a batch (training run). A training termination criterion was also used to stop simulation runs before the $50^{\text {th }}$ epoch, when the network produced zero error for all training images two times in a row. In this case, the number of epochs the networks required to complete during a batch was less than 50. These control settings were not obligatory for simulating the four networks in emergent. Either the four networks learned all the training images within 30 epochs or the learning curves stabilized at certain point between $20^{\text {th }}$ or $30^{\text {th }}$ epochs. Therefore, it was assumed that continuation of the simulation after $50^{\text {th }}$ epoch would only replicate similar results.

\subsubsection{Match between network-generated and expected outputs}

In the field of TC track and intensity forecasting, observed TC position and intensity in the TC best track data (JTWC, 2014; NOAA, 2015) are considered to be the most accurate information available about TC track and intensity. Therefore, comparing the networkgenerated track and intensity outputs with the observed track and intensity level in the TC

${ }^{3}$ All the input images used for training are presented to the four networks once during an epoch. 
best track data was considered as the best option for validating the four networks' performance in the last step.

In order to test different parameters quickly (see section 4.3.2), images of $60 \times 60$ pixels in size were used for training and testing the four networks. These low-resolution images limited producing TC movement forecasts to a maximum of eighteen possible directions, and seven intensity categories. The network-generated TC track and intensity forecasts were compared to the observed tracks $\left(360^{\circ}\right.$ was divided into 18 equal angles) and intensity categories (categorized wind speeds according to the Saffir-Simpson hurricane intensity scale) in the TC best track data on two separate datasets (see section 4.3.4) for accessing accuracy.

\subsubsection{Datasets used for training and testing}

Multi-source infrared, ocean heat content, sea-level pressure, wind direction and wind speed images of nine TCs formed in the Atlantic basin during the years 2008 and 2009 were used for TC track and intensity forecasting. The five types of images, infrared, ocean heat content, sea-level pressure, wind direction, and wind speed ${ }^{4}$ used for TC track and intensity forecasting (Figure 4-6) were recorded at 6-hour intervals; therefore, the number of images generated for each of the TCs varied depending on its lifecycle.

Five images recorded at a given time and the observed TC intensity category according to the TC best track data for that time plus forecast time offset was used as input-output pair for training and testing the two intensity-forecasting networks. Intensity forecasts were produced into seven intensity categories following the Saffir-Simpson hurricane intensity scale in the Atlantic basin (NHC (National Hurricane Center), 2013). The first and the second intensity categories represented tropical depression and tropical storm respectively. Intensity categories from three to seven represented category 1 through 5 hurricanes in the Saffir-Simpson scale.

The two intensity-forecasting networks were tested in two ways. First, the temporal image sequences belonging to the TCs were split randomly towards the end for preparing datasets for training and testing. The initial part of each sequence consisting of around $90 \%$ of the images of the sequence was used for training, whereas, the latter part consisting of around $10 \%$ of the images of the sequence (subsequently called late-phase images in this thesis) was used for testing the two intensity-forecasting networks (Table 4-7). These late phase images constituted the first type of images used for testing.

Table 4-7. Number of train and test images used for TC track and intensity prediction.

\begin{tabular}{|c|c|c|c|c|c|c|c|}
\hline \multirow{3}{*}{$\begin{array}{l}\text { TCs used for } \\
\text { this study }\end{array}$} & \multirow{3}{*}{$\begin{array}{c}\text { Images } \\
\text { recorded during }\end{array}$} & \multicolumn{6}{|c|}{ Number of input-output pairs } \\
\hline & & \multicolumn{2}{|c|}{$\begin{array}{l}\text { For } 6 \text { hour } \\
\text { forecast }\end{array}$} & \multicolumn{2}{|c|}{$\begin{array}{l}\text { For } 12 \text { hour } \\
\text { forecast }\end{array}$} & \multicolumn{2}{|c|}{$\begin{array}{l}\text { For } 24 \text { hour } \\
\text { forecast }\end{array}$} \\
\hline & & Train & Test & Train & Test & Train & Test \\
\hline AL 02 (2008), & $2008070312 *$ to & 240 & 30 & 120 & 15 & 56 & 7 \\
\hline
\end{tabular}

4 The author thanks John Knaff, research scientist at NOAA/NESDIS/Regional and Mesoscale Meteorology Branch, Fort Collins, Colorado, for providing him with infrared, ocean heat content, wind speed, and wind direction data. 


\begin{tabular}{|c|c|c|c|c|c|c|c|}
\hline \multirow{3}{*}{$\begin{array}{l}\text { TCs used for } \\
\text { this study }\end{array}$} & \multirow{3}{*}{$\begin{array}{c}\text { Images } \\
\text { recorded during }\end{array}$} & \multicolumn{6}{|c|}{ Number of input-output pairs } \\
\hline & & \multicolumn{2}{|c|}{$\begin{array}{l}\text { For } 6 \text { hour } \\
\text { forecast }\end{array}$} & \multicolumn{2}{|c|}{$\begin{array}{l}\text { For } 12 \text { hour } \\
\text { forecast }\end{array}$} & \multicolumn{2}{|c|}{$\begin{array}{l}\text { For } 24 \text { hour } \\
\text { forecast }\end{array}$} \\
\hline & & Train & Test & Train & Test & Train & Test \\
\hline Bertha & 2008071100 & & & & & & \\
\hline $\begin{array}{l}\text { AL } 04 \text { (2008), } \\
\text { Dolly }\end{array}$ & $\begin{array}{l}2008072106 \text { to } \\
2008072412\end{array}$ & 104 & 13 & 48 & 6 & - & - \\
\hline $\begin{array}{l}\text { AL } 07 \text { (2008), } \\
\text { Gustav }\end{array}$ & $\begin{array}{l}2008082518 \text { to } \\
2008090200\end{array}$ & 232 & 29 & 112 & 14 & 56 & 7 \\
\hline $\begin{array}{l}\text { AL 08 (2008), } \\
\text { Hanna }\end{array}$ & $\begin{array}{l}2008082812 \text { to } \\
2008090318\end{array}$ & 200 & 25 & 96 & 12 & 48 & 6 \\
\hline $\begin{array}{l}\text { AL } 09 \text { (2008), } \\
\text { Ike }\end{array}$ & $\begin{array}{l}2008090118 \text { to } \\
2008091006\end{array}$ & 272 & 34 & 136 & 17 & 64 & 8 \\
\hline $\begin{array}{l}\text { AL } 11 \text { (2008), } \\
\text { Kyle }\end{array}$ & $\begin{array}{l}2008092600 \text { to } \\
2008092912\end{array}$ & 104 & 13 & 56 & 7 & - & - \\
\hline $\begin{array}{l}\text { AL } 15 \text { (2008), } \\
\text { Omar }\end{array}$ & $\begin{array}{l}2008101406 \text { to } \\
2008101806\end{array}$ & 128 & 16 & 64 & 8 & 32 & 4 \\
\hline $\begin{array}{l}\text { AL } 17 \text { (2008), } \\
\text { Paloma }\end{array}$ & $\begin{array}{l}2008110518 \text { to } \\
2008110918\end{array}$ & 128 & 16 & 64 & 8 & 32 & 4 \\
\hline $\begin{array}{l}\text { AL } 03 \text { (2009), } \\
\text { Bill }\end{array}$ & $\begin{array}{l}2009081518 \text { to } \\
2009082118\end{array}$ & - & 207 & - & 96 & - & 45 \\
\hline
\end{tabular}

*First four digits indicate year, next two digits indicate months, next two digits indicate days and last two digits indicate hours.

Secondly, network performance was tested on a completely novel TC. The complete temporal image sequence recorded during the lifecycle of hurricane Bill constituted a second type of test. Out of the nine TCs, eight TCs formed during the year 2008 and Bill was the only TC that formed during the year 2009. As the lifecycles of hurricane Dolly (AL 04, 2008) and hurricane Kyle (AL 11, 2008) were short (would result in few training and test images), these two TCs were not used for 24-hour intensity forecast.

In addition to the five types of input images and the observed TC intensity levels, the observed TC movement direction and speed according to the TC best track data was used for training and testing the two combined TC track and intensity forecasting networks. Movement direction and movement speed forecasts were produced into 18 and 12 categories respectively. Unlike the two intensity-forecasting networks, the two combined TC track and intensity forecasting networks were tested only for the late-phase images (Table 4-7).

\subsubsection{Network structure}

The success in obtaining a reliable and robust network depends strongly on the choice of process variables involved as well as the available set of data and the domain used for training purposes (Despagne \& Massart, 1998). A three-step approach was followed to construct valid networks for TC track and intensity forecasting in this research (see section 4.3). The number of variables used for prediction and the number of variables to be predicted is represented by the number of input layers used and the number of output layers respectively (Kasiri, Aleboyeh, \& Aleboyeh, 2008). 
The two intensity-forecasting networks processed inputs at four hierarchical levels. The first and the last processing levels in the hierarchy constituted the input and the output respectively. Five separate layers were used at the input level to feed the five different types of images into the two networks in parallel. Thus, five input layers, two hidden layers (V1V2 and V4-IT) and one output layer (called intensity) constituted the basic structure of the two intensity-forecasting networks in this research (Figure 4-7).
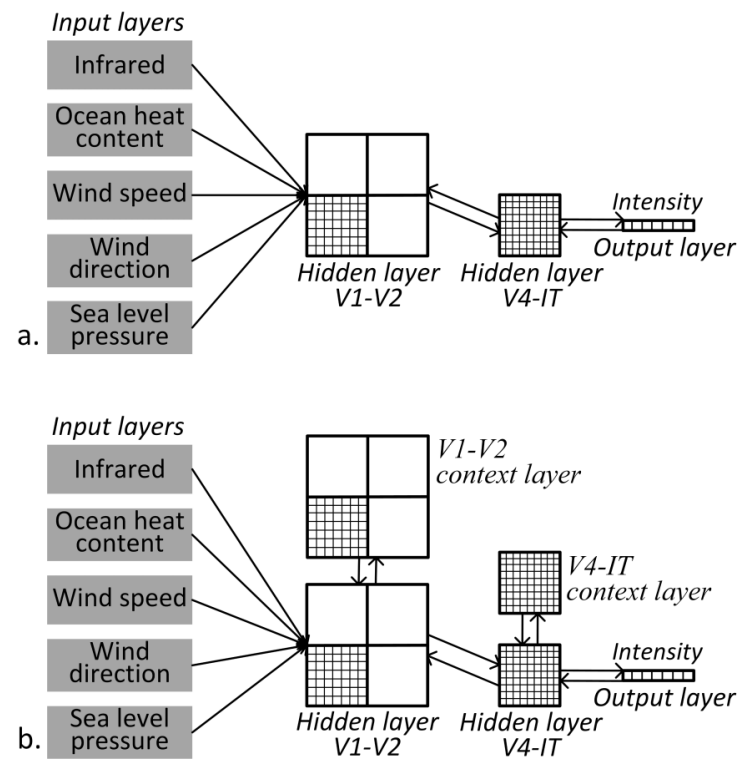

Figure 4-7. The two networks used for intensity forecasting. a. Network without context layers and $\mathbf{b}$. network using context layers.

The two intensity-forecasting networks were identical, but to store prior time step's hidden layer activity and use that information for interpreting the current image, two additional layers (called V1-V2 context and V4-IT context) were used in one of the two networks (Figure 4-7b). These two additional layers allowed the network to handle temporal pattern of intensity change in a way, which is similar to Markov chain (O'Reilly \& Munakata, 2000). Structure and important features of the layers are described in the list below:

1. Each input layer was $60 \times 60$ pixels in size and was divided into four parts $(32 \times 32$ pixels each). These parts worked as RFs for the units in the V1-V2 layer.

2. In V1-V2 hidden layer, units were arranged into $2 \times 2$ groups. Each of these groups contained $7 \times 7$ units and received inputs from an area covering $32 \times 32$ pixels in all the five input layers (RFs). Units in the same group had same RF and all four groups together covered the entire input layer. The RFs were also allowed to overlap at the edges by two pixels in order to maintain continuity of the images during processing.

3. V4-IT hidden layer contained $9 \times 9$ units. All the units in this layer received combined information from all the groups in V1-V2 layer. Thus, units in V4-IT hidden layer received information from all the parts in the five input layers. 
4. The V1-V2 and V4-IT context layers contained the same number of units as V1-V2 and V4-IT hidden layers respectively. These layers were bi-directionally connected to the hidden layers. Connections from the hidden layers to the context layers were one-to-one that means activations were allowed transfer from a certain unit in the hidden layer to the corresponding units in the context layer. For example, from the lower left unit in the lower left group of V1-V2 hidden layer to the corresponding unit in the V1-V2 context layer. Connections from the context layers to the hidden layers were many-to-many that means all the units in the context layers sent activations to all the units in the hidden layers.

5. A vector of $7 \times 1$ units in size represented the intensity layer. Each unit of this vector indicated one of the seven intensity levels of the Saffir-Simpson hurricane intensity scale. The unit at the left end of this vector represented the intensity of a tropical depression and the unit at the right end represented the intensity of a category five TC.
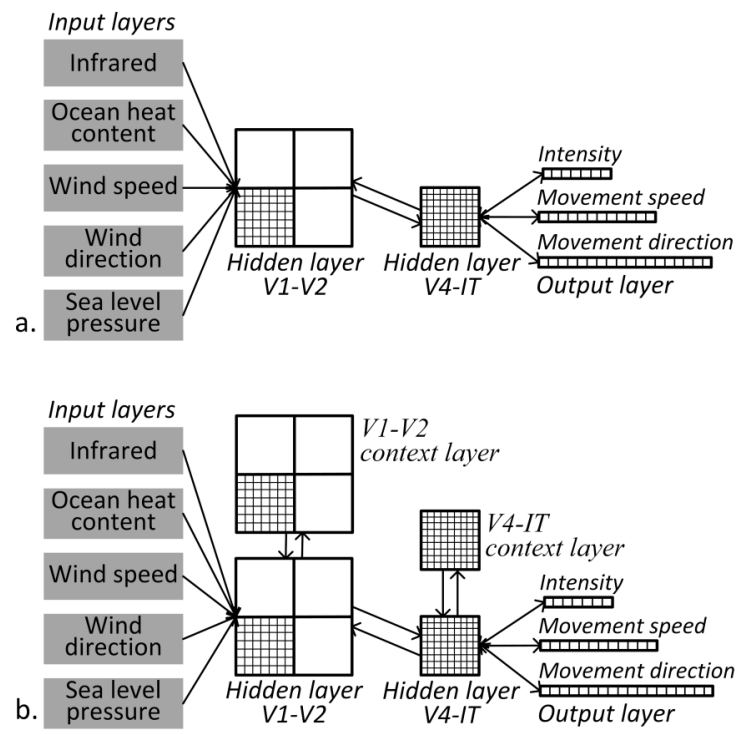

Figure 4-8. Networks for combined track and intensity forecasting, a. network without context layers and $\mathbf{b}$. network using context layers.

Structure of the two combined TC track and intensity forecasting networks were identical to the two intensity-forecasting networks (Figure 4-8), except that two additional output layers, movement direction and movement speed were used to produce forecasts for TCs' movement direction and speed of TCs' location change respectively. A single output layer was enough to produce forecasts for TC intensity, but for forecasting track, both movement direction and movement speed information were necessary. Movement direction denoted, in which direction a TC might move in the future (during the next 12 hours in this case), whereas, movement speed provided information about, at which speed (in $\mathrm{km} / \mathrm{h}$ ) the TC changed its location in that direction, which finally provided the distances the TC is expected to cross during the next 12 hours. 


\subsubsection{Information processing in the network}

Information processing in the network started with transferring of weighted sum of inputs from the input layers to the first hidden layer. How strongly a unit should react to input can be expressed by a biologically based sigmoid activation function. The four networks processed each input image through two activation phases namely, minus and plus phases. In the minus phase, the networks processed each input through the layers and produced a track or an intensity forecast by settling down into an activation state (activations ranging between 0 and 1) that was consistent with that particular input and the current set of weights. To achieve inhibitory competition among the units within a group and/or within a layer the kWTA inhibitory function was used (O'Reilly \& Munakata, 2000). In the plus phase, the four networks' activations were reset and both the input and the correct output were presented to the networks. This allowed all the hidden layer units of the four networks to reach an activation state (ranging between 0 and 1) that was required for producing the correct output.

The minus and the plus phase activations of each unit were compared and the activation error produced by each unit was calculated. This error was gradually decreased during training by adjusting the receiving weights for each unit according to the activation error (error-driven or supervised part of the learning). Moreover, a weight change was also computed on the basis of the co-activation pattern of paired units (correlation-based or unsupervised part of the learning). During training, a positive weight change was assigned to encourage co-activated pairs, whereas a negative weight change was assigned, if receiving unit in a pair was active but the sending unit was inactive. Any weight change did not take place in cases, when the receiving unit in a pair was inactive (O'Reilly \& Munakata, 2000). The resultant weight of each connection remained within the interval 0 and 1, which was obtained through combining the error-driven and the correlation-based Hebbian learning components and normalizing the weights after each update cycle.

\subsubsection{Training of the networks}

Input-output pairs used to train the four networks were stored in a data-table as rows. In the two intensity-forecasting networks, each row consisted of five $60 \times 60$ (for the five input images) and one $7 \times 1$ (for output) matrices. In the two combined track and intensity forecasting networks, though size of the matrices used for the five inputs was $60 \times 60$, three different matrices of size $18 \times 1$ (movement direction), $12 \times 1$ (movement speed), and $7 \times 1$ (intensity) were used for three different outputs. In the beginning of the simulation, random weights were assigned to all the connections in the four networks. The networks were trained in epochs using the settings presented in Table 4-8. During each epoch, all the training images of the same $\mathrm{TC}$ were presented to the four networks in a sequential (temporal) order. A learning rate schedule was used for all the four networks to gradually decrease the initial learning rate of 0.01 during training, in this way speeding up initial weight changes, while helping the weights to better stabilize towards the final stages of training.

The four networks' performance during training was measured as the difference between the output generated by the networks and the correct output (movement direction/intensity) for that particular image provided in the training data set. This error was calculated for all the output units, squared for handling sign differences, and finally summed to produce overall summed squared error (SSE) each time an input-output pair was presented to the 
four networks. SSEs produced by the networks in the course of processing all input-output pairs in the training set were averaged to get an average SSE at the end of each epoch.

An additional measure of error that was used was 'count error'. This measure differs from SSE in that it simply counts the cases where the output is wrong, not taking into consideration the amount of deviation from the target output. In other words, if the correct output unit was activated above 0.5 , and all other output units were below 0.5 in activation, the output was considered to be correct. In all other cases, the output was counted as incorrect.

Table 4-8. Datasets and settings used for training the four networks.

\begin{tabular}{|c|c|c|c|c|c|c|c|c|}
\hline \multicolumn{9}{|c|}{ Training of the two intensity-forecasting networks } \\
\hline \multirow[t]{2}{*}{$\begin{array}{c}\text { Forecast } \\
\text { length }\end{array}$} & \multirow[t]{2}{*}{$\begin{array}{c}\text { Dataset } \\
\text { used }\end{array}$} & \multicolumn{4}{|c|}{$\begin{array}{c}\text { Number of input-output pairs, } \\
\text { when the training datasets } \\
\text { consisted images of }\end{array}$} & \multirow[t]{2}{*}{$\begin{array}{c}\text { Maximum } \\
\text { epochs }\end{array}$} & \multirow{2}{*}{$\begin{array}{l}\text { Number } \\
\text { of } \\
\text { training } \\
\text { runs }\end{array}$} & \multirow[t]{2}{*}{$\begin{array}{l}\text { Training } \\
\text { stopping } \\
\text { criterion }\end{array}$} \\
\hline & & 4TCs & $5 T C s$ & 6TCs & 7TCs & & & \\
\hline 6 hour & $\begin{array}{l}\text { 6-hour } \\
\text { forecast }\end{array}$ & 776 & - & 1152 & 1280 & 50 & 10 & $\begin{array}{l}0 \text { error } \\
\text { two times } \\
\text { in a row }\end{array}$ \\
\hline 12 hour & $\begin{array}{l}\text { 12-hour } \\
\text { forecast }\end{array}$ & - & 512 & 568 & 635 & 50 & 10 & $\begin{array}{l}0 \text { error } \\
\text { two times } \\
\text { in a row }\end{array}$ \\
\hline 24 hour & $\begin{array}{l}\text { 24-hour } \\
\text { forecast }\end{array}$ & 224 & 256 & - & - & 50 & 10 & $\begin{array}{l}0 \text { error } \\
\text { two times } \\
\text { in a row }\end{array}$ \\
\hline
\end{tabular}

Training of the two combined track and intensity forecasting networks

\begin{tabular}{|c|c|c|c|c|c|c|}
\hline \multirow[t]{2}{*}{$\begin{array}{l}\text { Forecast } \\
\text { length }\end{array}$} & \multirow[t]{2}{*}{$\begin{array}{l}\text { Dataset } \\
\text { used }\end{array}$} & \multicolumn{2}{|c|}{$\begin{array}{c}\text { Number of input-output pairs, } \\
\text { when the training datasets } \\
\text { consisted images of }\end{array}$} & \multirow[t]{2}{*}{$\begin{array}{c}\text { Maximum } \\
\text { epochs }\end{array}$} & \multirow{2}{*}{$\begin{array}{c}\text { Number } \\
\text { of } \\
\text { training } \\
\text { runs }\end{array}$} & \multirow[t]{2}{*}{$\begin{array}{l}\text { Training } \\
\text { stopping } \\
\text { criterion }\end{array}$} \\
\hline & & 7TCs & 8TCs & & & \\
\hline 12 hour & $\begin{array}{l}\text { 12-hour } \\
\text { forecast }\end{array}$ & 635 & 699 & 50 & 10 & $\begin{array}{l}0 \text { error } \\
\text { two times } \\
\text { in a row }\end{array}$ \\
\hline
\end{tabular}

As the four networks learned to correctly interpret more and more input images during successive epochs of each batch (training run), both the average SSE and the number of images for which the networks produced a wrong prediction (count error) decreased gradually. At the end of each training run, the four networks stopped updating the weights, saved the final weights, and initialized random weights to all the connections, so that another training run could take place. Each of the four networks was trained for 10 batches to make sure that the networks' learning performance was not influenced by the random initial weights. The weights that were saved at the end of each training run were later used for initializing the four networks' weights when starting up testing, and were not used for incremental training.

\subsubsection{Training performance}

Training performance of the four networks during individual training run was stable and not dependent on initial random weight. Of the two intensity-forecasting networks, the network without context layers learned faster and performed little better compared to the network 
using context layers, when the networks were separately trained using the 6-hour, 12-hour, or the 24-hour forecast datasets of different number of TCs (Figure 4-9).

The learning curve reached zero count error after around 35 epochs, when the intensityforecasting network without context layers was trained using the 6-hour forecast datasets of four TCs (Figure 4-9a). When the same network was trained using the 6-hour forecast datasets of six and seven TCs, the learning curves stabilized at 2 count error after around 20 epochs (Figure 4-9a). The learning curves went down to zero within 25 epochs, when the 12-hour forecast datasets of five, six, and seven TCs were used for training (Figure 4-9b). The learning curves went down to zero within 10 epochs, when the 24-hour forecast datasets of four and five TCs were used for training (Figure 4-9c).
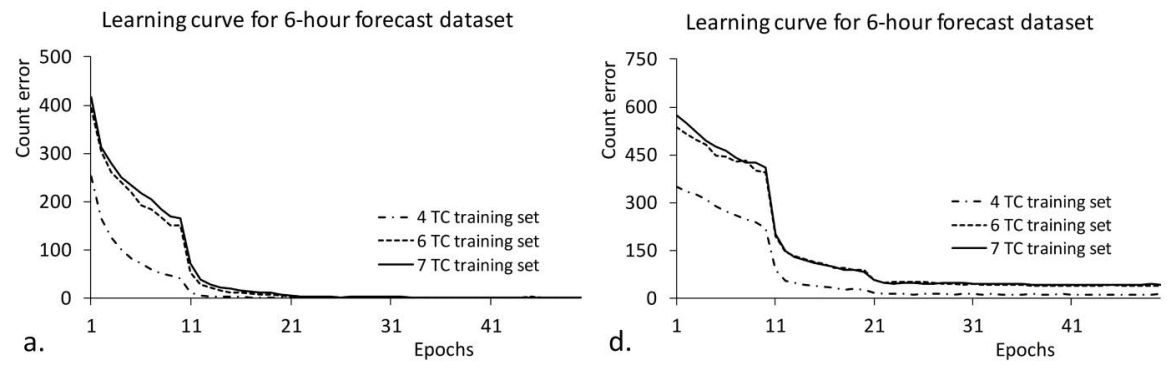

Learning curve for 12-hour forecast dataset

Learning curve for 12-hour forecast dataset

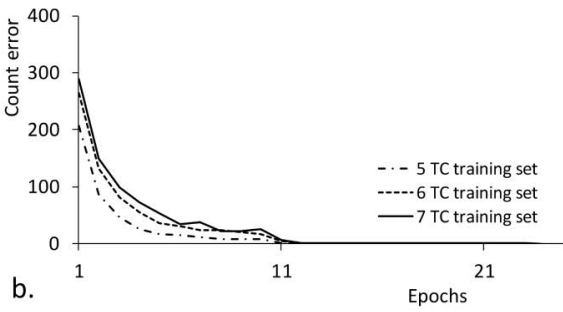

Learning curve for 24-hour forecast dataset

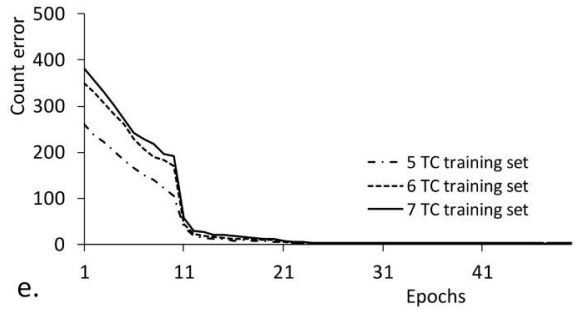

Learning curve for 24-hour forecast dataset
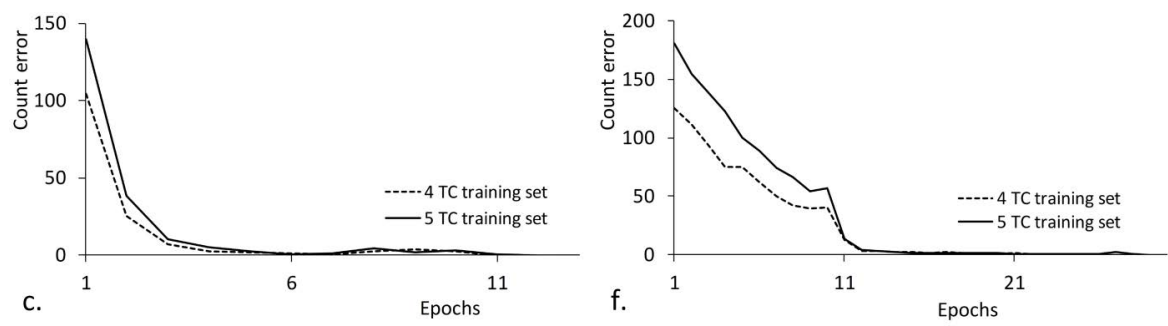

Figure 4-9. Training performance of the two intensity-forecasting networks. Figures a. through c. illustrate learning curves produced by the network without context layers for 6-, 12-, and 24-hour forecast datasets. Figures d. through f. illustrate learning curves produced by the network using context layers for 6-, 12-, and 24-hour forecast datasets.

Training error decreased gradually up to 20 epochs and stabilized at 12 count error, when the intensity-forecasting network using context layers was trained using the 6-hour forecast datasets of four TCs (Figure 4-9d). The training error was greater when images of six and seven TCs were used for training (Figure 4-9d). For the 12-hour forecast datasets of five, six, and seven TCs, the learning curves reached at around 30 count error after 10 epochs, 
then gradually reached at around 2 count error after 20 epochs and stabilized there for rest of the training (Figure 4-9e). The learning curves reached zero within 30 epochs, when 24hour forecast datasets of four and five TCs were used for training (Figure 4-9f).

It has previously been mentioned that the two combined TC track and intensity forecasting networks were trained only for producing 12-hour forecast. Learning performance of the two networks were stable, when the two networks were trained using 12-hour forecast datasets of seven and eight TCs (Figure 4-10). Both the SSE and count error produced by the network without context layers decreased rapidly within 15 epochs, then stabilized at around 0.03 and 5 respectively during rest of the training (Figure 4-10a and b). Learning performance of the network using context layers was almost the same as of the network without context layers (Figure 4-10c and d). SSE and count error decreased sharply within 14 epochs and then stabilized at around 0.01 and 1 respectively during continued training.
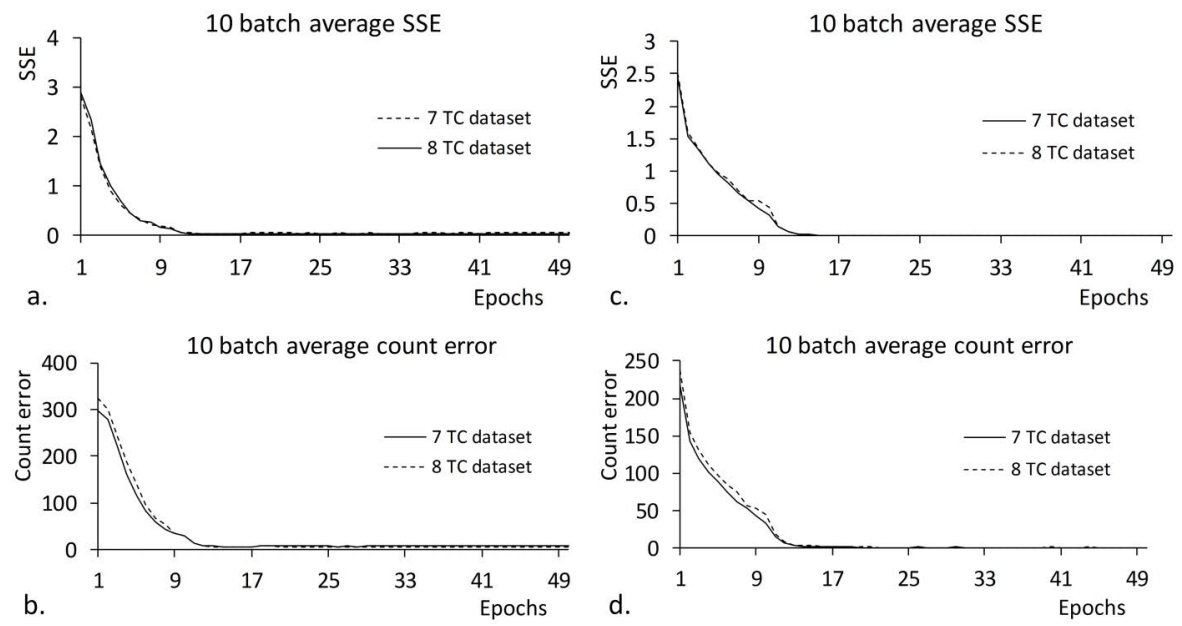

Figure 4-10. Learning performance of the two networks used for combined TC track and intensity forecasting. Figures $\mathbf{a}$. and $\mathbf{b}$. illustrate learning curves produced by the network without context layer, whereas figures $\mathbf{c}$. and d. illustrate learning curves produced by the network using context layers.

\subsubsection{Activation-based receptive field analysis}

To give an insight into how feature detection works in the four networks, an Activationbased RF (ActRF) analysis was conducted on the units in the upper-right V1-V2 group of the two intensity-forecasting networks (Figure 4-11). ActRF analysis reveals how an individual unit's activity in the analyzed layer is correlated with the activation patterns in the five input layers and the output layer. When analyzing V1-V2 hidden layer with respect to the input and output layers, the analysis shows, which input patterns a feature detector has specialized for, and which TC intensity output that a particular feature detector unit contributes to.

The activation patterns visualized in Figure 4-11 show the average input favored by the detectors (units in the upper-right group) in the V1-V2 hidden layer, measured across all the inputs. Each square in Figure 4-11 represents the input as seen by a particular unit in the 
analyzed V1-V2 group. As can be seen, there were 7 x 7 units in the V1-V2 group. The upper-right corners of the squares in Figure 4-11 are marked using white lines. This is the portion of input that a unit in the V1-V2 hidden layer actually sees input from. The activity patterns within these sub-squares represent the features that the particular unit has learned to favor. Some units in this group learned to react to very specific features associated with a few input images, which resulted in discrete activation preferences in Figure 4-11.
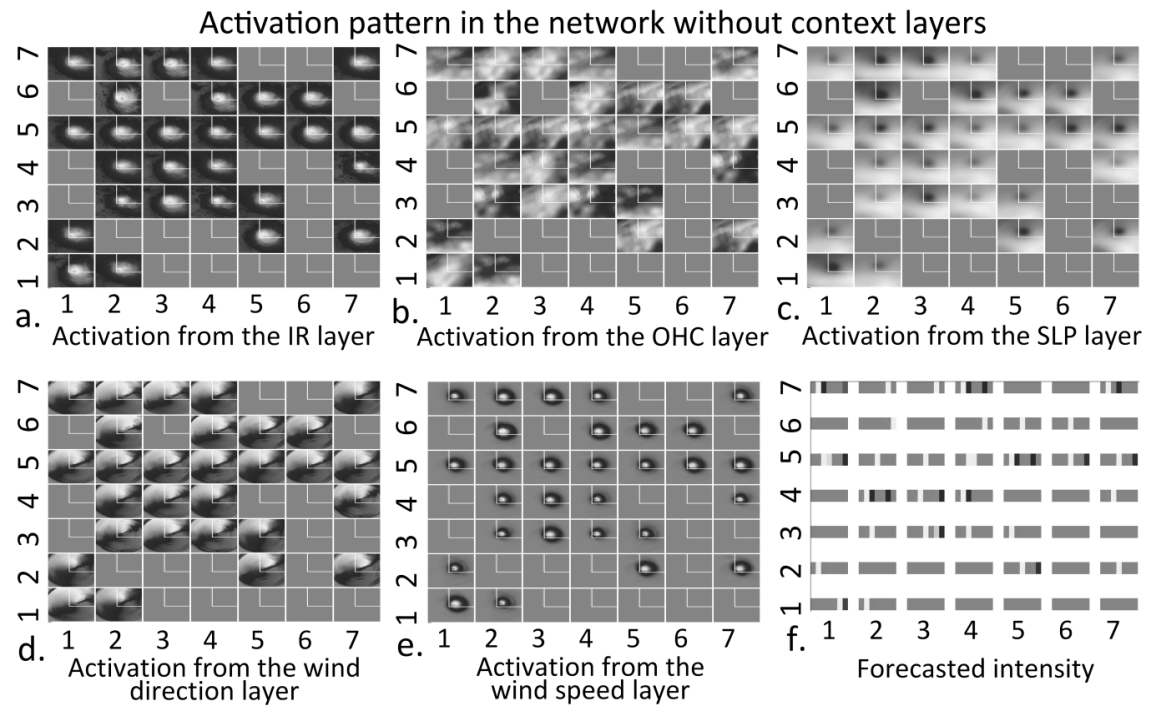

Activation pattern in the network using context layers

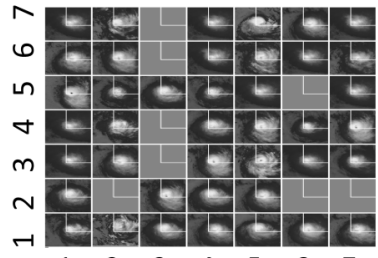

a. $\begin{array}{lllllll}1 & 2 & 3 & 4 & 5 & 6 & 7\end{array}$ Activation from the IR layer

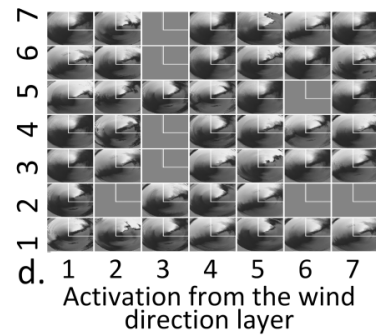

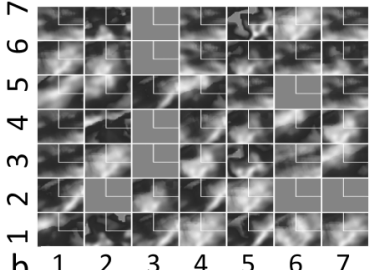

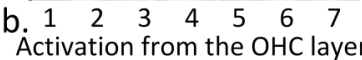

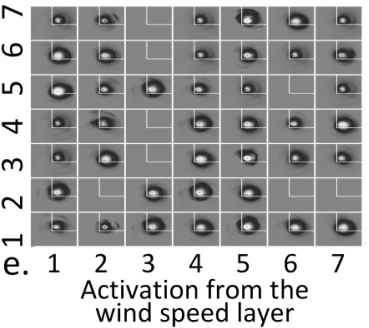

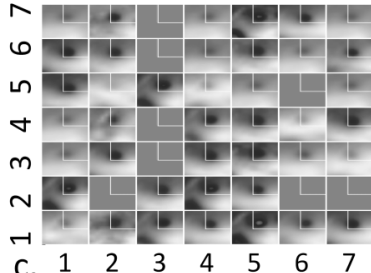

Activation from the SLP layer

Figure 4-11. a. through e. illustrate activations in the input layer averaged over cases, when the receiving units in the upper-right group of the V1 layer became activated by all the input images

(12-hour datasets of six TCs) during training, and f. illustrates the forecasted intensity level produced by each unit in this group. Each square in this figure illustrates the input as seen by a particular unit in the analyzed V1 group. As can be seen, there were $7 \times 7$ units in the V1 group. 
Activations received by the units in the upper-right V1-V2 group from the infrared layer indicate that these units learned to react to features that are common in all the infrared images. Elongated shapes in Figure 4-11a, seems to be associated with low TC-intensity level, whereas spherical shapes in Figure 4-11a, appear to be related to higher TC-intensity levels in the output layer (Figure 4-11f).

Clusters of highly activated (bright) pixels in the centers of the squares in Figure 4-11b seem indicate high ocean heat content. This type of activation pattern is also associated with higher TC-intensity levels in Figure 4-11f. The presence of more lowly activated (dark) pixels as well as non-clustered bright pixels in the activation patterns most likely to specify low ocean heat content, which are also associated with low TC-intensity levels.

Low central pressure levels usually characterize intense TCs. Activation patterns containing more lowly activated pixels in Figure 4-11c are representative of low sea level pressure. These patterns are also associated with higher TC-intensity levels, whereas patterns containing more highly activated pixels seem to be correlated to low TC-intensity levels in Figure 4-11f.

In the wind direction images, pixel values represent movement directions (in degrees) of wind surrounding the TCs. However, to link these features with output TC intensity levels, first it is necessary to know the wind circulation pattern within a TC. In the northern hemisphere, wind travels counterclockwise around a low-pressure center. Therefore, winds blowing towards west and northwest usually arise in the north and north-east corner of a low-pressure center in an intense TC. These west or northwest blowing winds have a movement direction of $>270^{\circ}$, whereas winds blowing towards south or east have movement directions, which is $<270^{\circ}$. In the wind direction features (Figure 4-11d), west or north-west blowing winds are represented by highly activated pixels, whereas winds blowing towards other directions are represented by lowly and moderately activated pixels. The presence of highly activated pixels in the north and north-east corner is noticeable in the activation patterns, which are associated with high intensity levels. Activation patterns where highly activated pixels are not present in that corner seem to be associated with low intensity levels in Figure 4-11f.

Activation patterns containing broader areas highly activated pixels in Figure 4-11e seem to be associated with high wind speed and thus with higher TC-intensity levels in Figure 4-11f. Activation patterns consisting of smaller areas of moderate wind speed (represented by few highly activated and more lowly activated pixels) appear to be associated with low TC-intensity levels.

\subsubsection{Testing of the networks}

The two intensity-forecasting networks were separately tested for two types of test datasets (Table 4-9). In contrast, the two combined TC track and intensity forecasting networks were tested for the 12-hour forecast test datasets consisting of late-phase images (images recorded during the end of TC lifecycles) of seven or eight TCs (Table 4-9).

As learning did not take place during testing, the four networks' performance for the test datasets was dependent on the final weights saved during individual training runs. All the weights saved during the individual training runs were used to initialize the four networks' weights during testing. Network performance during testing was measured using the same gauges as in network training (SSE and count error). 
Table 4-9. Datasets used for testing the four networks.

\begin{tabular}{|c|c|c|c|c|c|c|}
\hline \multicolumn{7}{|c|}{ Datasets used for training the two intensity-forecasting networks } \\
\hline \multirow[t]{2}{*}{$\begin{array}{c}\text { Forecast } \\
\text { length }\end{array}$} & \multirow[t]{2}{*}{$\begin{array}{c}\text { Dataset } \\
\text { used }\end{array}$} & \multicolumn{4}{|c|}{$\begin{array}{l}\text { Number of input-output } \\
\text { pairs, when the test datasets } \\
\text { consisted late-phase images } \\
\text { of }\end{array}$} & \multirow[t]{2}{*}{$\begin{array}{l}\text { Number of input-output } \\
\text { pairs, when the test } \\
\text { datasets consisted } \\
\text { images of novel TC Bill }\end{array}$} \\
\hline & & 4TCs & $5 \mathrm{TCs}$ & 6TCs & 7TCs & \\
\hline 6 hour & $\begin{array}{l}\text { 6-hour } \\
\text { forecast }\end{array}$ & 97 & - & 144 & 160 & 207 \\
\hline 12 hour & $\begin{array}{l}\text { 12-hour } \\
\text { forecast }\end{array}$ & - & 64 & 71 & 79 & 96 \\
\hline 24 hour & $\begin{array}{l}24-\text { hour } \\
\text { forecast }\end{array}$ & 32 & 28 & - & - & 45 \\
\hline \multicolumn{7}{|c|}{$\begin{array}{c}\text { Datasets used for training the two combined track and intensity forecasting } \\
\text { networks }\end{array}$} \\
\hline \multirow[t]{2}{*}{$\begin{array}{l}\text { Forecast } \\
\text { length }\end{array}$} & \multirow[t]{2}{*}{$\begin{array}{l}\text { Dataset } \\
\text { used }\end{array}$} & \multicolumn{5}{|c|}{$\begin{array}{l}\text { Number of input-output pairs, when the test datasets } \\
\text { consisted late-phase images of }\end{array}$} \\
\hline & & & $7 \mathrm{~T}$ & & & 8TCs \\
\hline 12 hour & $\begin{array}{l}\text { 12-hour } \\
\text { forecast }\end{array}$ & & 79 & & & 87 \\
\hline
\end{tabular}




\section{Chapter 5}

\section{Results}

\subsection{Results elicited from warning providers}

The answers obtained during the interview among the meteorologists at BMD are provided in the next three subsections. These subsections also correspond to the three main themes in Table 4-3.

\subsubsection{TC forecasting}

BMD has access to data recorded using ground, ocean, air, and space borne instruments (Table 5-1). Despite the availability of high-quality meteorological data, BMD is only using wind speed and wind direction information for forecasting TCs in the Bay of Bengal (BoB). The meteorologists at BMD use radar and satellite recorded images, but these images are only used for getting TCs' current position and not as inputs to the TC forecasting techniques.

Table 5-1. Data availability and data used for TC forecasting.

\begin{tabular}{llcc}
\hline \multicolumn{1}{c}{ Instruments } & \multicolumn{1}{c}{ Data Type } & \multicolumn{1}{c}{$\begin{array}{c}\text { Availability } \\
(\mathbf{Y} / \mathbf{N})\end{array}$} & $\begin{array}{c}\text { Used for TC } \\
\text { forecasting } \\
\text { (Y/N) }\end{array}$ \\
\hline Ground based & Temperature & $\mathrm{Y}$ & $\mathrm{N}$ \\
\cline { 2 - 4 } & Humidity & $\mathrm{Y}$ & $\mathrm{N}$ \\
\cline { 2 - 4 } & Rainfall & $\mathrm{Y}$ & $\mathrm{N}$ \\
\cline { 2 - 4 } & Pressure & $\mathrm{Y}$ & $\mathrm{N}$ \\
\cline { 2 - 4 } & $\begin{array}{l}\text { Wind speed and } \\
\text { direction }\end{array}$ & $\mathrm{Y}$ & $\mathrm{Y}$ \\
\cline { 2 - 4 } & Solar radiation & $\mathrm{Y}$ & $\mathrm{N}$ \\
\cline { 2 - 4 } & Radar & $\mathrm{Y}$ & $\mathrm{Y}$ \\
\hline $\begin{array}{l}\text { Ocean based } \\
\text { (Ocean buoy) }\end{array}$ & $\begin{array}{l}\text { meteorological } \\
\text { and sea surface } \\
\text { data }\end{array}$ & $\mathrm{Y}$ & $\mathrm{Y}$ \\
\hline $\begin{array}{l}\text { Upper air } \\
\text { (weather balloon) }\end{array}$ & $\begin{array}{l}\text { Wind speed and } \\
\text { direction }\end{array}$ & $\mathrm{Y}$ & $\mathrm{Y}$ \\
\hline Space based & $\begin{array}{l}\text { Weather satellite } \\
\text { image }\end{array}$ & $\mathrm{Y}$ & \\
\hline
\end{tabular}

Once data from ground-based weather stations and weather balloons are available, the meteorologists at BMD produce TC forecasts in three successive steps (Figure 5-1): 
1. In the first step, wind data are interpolated to produce a map of continuous wind fields showing wind speed and wind direction. The current and recent past locations of the current TC are also plotted on the same map. These wind data are analyzed using Buys Ballot law (Roulstone \& Norbury, 2013) to better realize the synoptic condition associated with the approaching TC.

2. In the second step, wind speed and direction within the TC vortex are compared with wind direction and speed in the surrounding environment to determine the pressure level (steering level), where environmental winds match best with the winds within the TC vortex.

3. TC forecasts are produced in the final step, where meteorologists take advantages of three different techniques separately: (a) steering airflow determination (Keenan, 1982), (b) averaging across historical TCs (Jeffries et al., 1993), and (c) climatology and persistence (Neumann, 1972). Though these techniques are separately used for producing TC forecasts, meteorologists ultimately rely on their expert knowledge to forecast TC track and intensity for the next 12 hours on the basis of the movement direction and speed of the analogous past TCs and the extrapolated movement of the current TC.

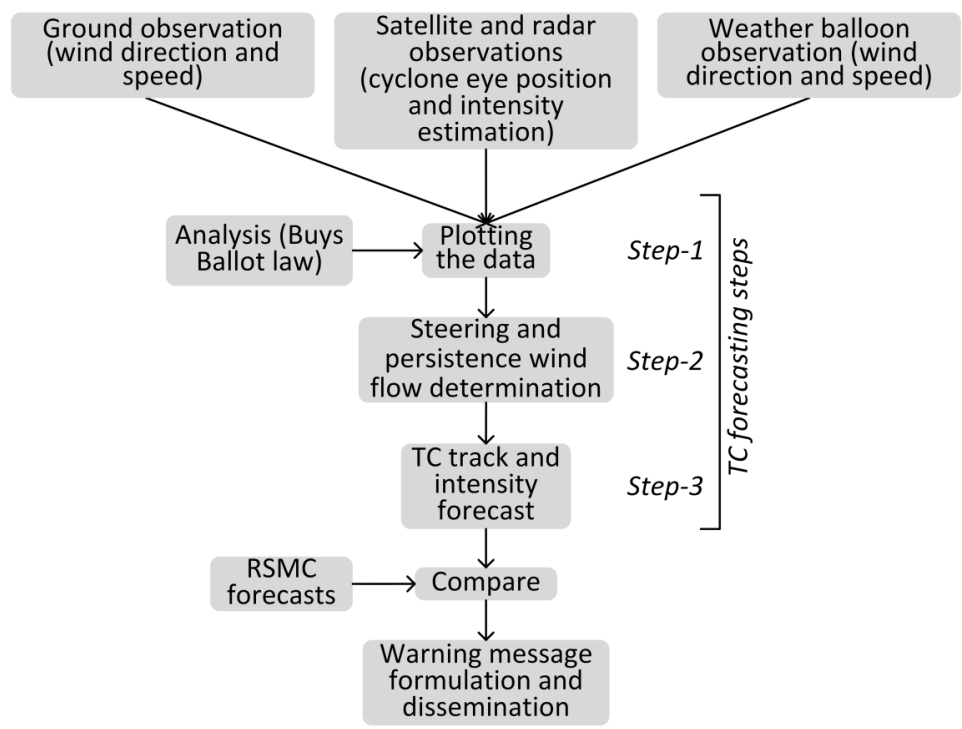

Figure 5-1. Flowchart of TC forecasting at BMD prepared based on the flowcharts drawn by the meteorologists during the interview.

\subsubsection{Warning message formulation and dissemination}

BMD receives numerical-model-generated forecasts from RSMC (Regional Specialized Meteorological Center) in India as a member state of the World Meteorological Organization (WMO), but the meteorologists at BMD always emphasize self-produced forecasts during the formulation of warning messages. In the warning messages, two separate signaling systems are used for the maritime and the river ports to express the 
danger levels associated with TCs. An 11-point signaling system (Appendix B), where 1 corresponds to the lowest and 11 corresponds to the highest danger level, is used for the maritime ports. In contrast, a 4-point signaling system (Appendix C), where signals 1 and 4 refer to the lowest and the highest danger levels, respectively, is used for the river ports. The current TC signaling system at BMD was introduced during the British colonial period. In this system, the coastal districts located to the west of the Meghna estuary are considered as the command area of the Mongla maritime port, while districts located to the east are considered as the command area of the Chittagong maritime port. Maritime ports and their command areas always receive the same signal (danger level) in the warning message (Appendix F).

Once the warning message is formulated, BMD disseminates it to the maritime ports, the river ports, and the media. BMD also sends the warning message directly to the National Coordination Committee, the cyclone preparedness program, the relief and rehabilitation authorities and to the local administrations (Akhand, 2003; Debsarma, 2001). BMD disseminates new warning messages as special weather bulletins at every three to six hours, depending on the severity and motion characteristics of the approaching TC (SWC (Storm warning Center), 2007, 2013).

\subsubsection{Limitations and future development plans}

Data scarcity over the $\mathrm{BoB}$ is one of the major problems that BMD is currently struggling with (Table 5-2). Wind speed and direction data recorded by ground-based stations are available every 6-hours, whereas upper-level wind speed and direction data recorded by weather balloons are only available every $12-$ hours. TCs in the BoB spend most of their lifespans outside the coverage of these weather stations as well as outside the coverage of the two radars installed in the coastal area of Bangladesh (Figure 5-2). Therefore, the meteorologists have to use interpolated values of 12-hour old upper atmospheric wind information to produce forecasts for TCs.

BMD has access to ocean buoy as well as NCEP (Kalnay et al., 1996) and ECMWF (Simmons, Uppala, Dee, \& Kobayashi, 2007) atmospheric reanalysis data, which could solve the problem of data scarcity over the BoB. However, the currently used TC forecasting techniques do not support the formats of these data. In addition, the meteorologists at BMD do not have the necessary expertise to re-code and use these data as inputs to the operational TC forecasting techniques for producing better quality forecasts.

The three TC forecasting techniques that the meteorologists at BMD take advantages of are not capable of producing good accuracy forecasts (Jeffries et al., 1993; Roy \& Kovordányi, 2012, 2015). This means the meteorologists at BMD ultimately have to rely on their expert knowledge for producing TC track and intensity forecasts. As the meteorologists do not use any forecast verification, it is not possible for them to include the precision level of the produced forecasts in the disseminated warning messages (Appendix F). Moreover, too many danger-level options in the current signal system make the warnings difficult to understand by the residents in the coastal areas (U. Haque et al., 2012; Miyan, 2006).

BMD currently runs four numerical techniques for producing forecasts for storm surge and rainfall. These techniques are capable of using data obtained from multiple sources as predictors, therefore, could be transformed into effective TC forecasting techniques with necessary adjustments. As the meteorologists do not have necessary data-processing skills 
and computational knowledge to modify these techniques, producing good accuracy forecasts for new atmospheric phenomenon like TC seems not possible.

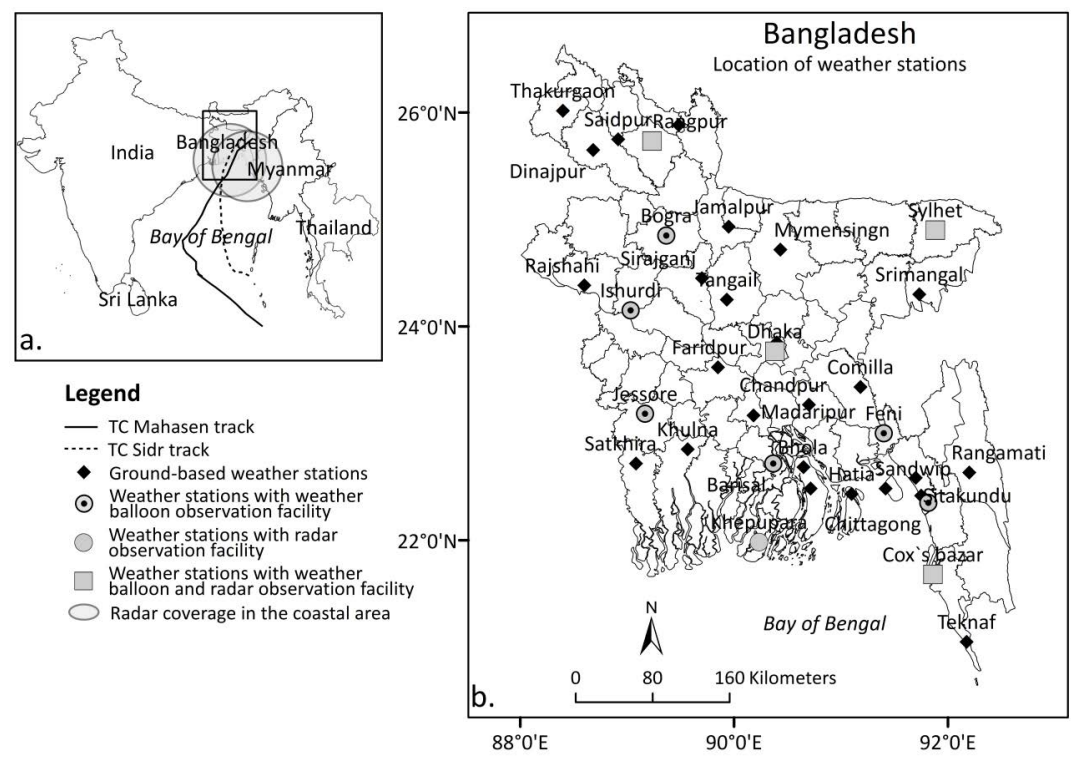

Figure 5-2. a. Illustrates track of the two studied TCs. The two circles in illustrate the coverage of the two radars that are installed in the coastal area. Figure b. illustrates locations of ground-based weather stations, ground-based radar stations, and weather balloon stations in Bangladesh.

During the interview, the three meteorologists proposed a number of plans for improving the effectiveness of cyclone early warning system in the future (Table 5-2).

Table 5-2. Limitations of TC forecasting and warning at BMD and plans suggested by the meteorologists for improving effectiveness of cyclone early warning system in the future.

\begin{tabular}{ll}
\hline \multicolumn{1}{c}{ Limitations } & \multicolumn{1}{c}{ Future development plans } \\
\hline $\begin{array}{l}\text { Data scarcity over the Bay of } \\
\text { Bengal }\end{array}$ & $\begin{array}{l}\text { Using new data for better coverage over the } \\
\text { BoB, such as: } \\
\text { - ocean buoy data, and } \\
\text { - atmospheric reanalysis data products. }\end{array}$ \\
\hline $\begin{array}{l}\text { Quality of the forecast and } \\
\text { forecast verification }\end{array}$ & $\begin{array}{l}\text { Deploying the hurricane-forecasting version of } \\
\text { the Weather Research and Forecasting (HWRF) } \\
\text { model for precise long-term TC track and } \\
\text { intensity forecasts. }\end{array}$ \\
\hline $\begin{array}{l}\text { Lack of expertise and data } \\
\text { integration }\end{array}$ & $\begin{array}{l}\text { - Further training to allow the meteorologists to } \\
\text { handle new types of data and numerical } \\
\text { weather prediction models. }\end{array}$ \\
\hline $\begin{array}{l}\text { Difficult to understand danger } \\
\text { levels in the warning messages }\end{array}$ & $\begin{array}{l}\text { Deploying generalized signaling system for the } \\
\text { maritime and river ports (Appendix G). }\end{array}$ \\
\hline $\begin{array}{l}\text { Insufficient computational } \\
\text { resources }\end{array}$ & Scaling up the computational resources. \\
\hline
\end{tabular}




\subsection{Results elicited from warning receivers}

The answers obtained from the 200 respondents in the questionnaire survey are provided in the next five subsections.

\subsubsection{Warning message reception}

$90.5 \%$ of the 200 respondents received warnings during Sidr, and this number increased to $96 \%$ during Mahasen. The remaining respondents did not receive warning messages mainly due to: (a) did not have access to radio or television, and/or (b) lived in remote areas, and therefore could not be reached by megaphones.

Various media's role in warning message dissemination among the participants: (a) the media through which they received warning messages during the two TCs, (b) media through which they would like to receive warning messages, and (c) reasons for preferring those media are presented in Table 5-3.

Table 5-3. Media from which the respondents received warning messages, media from which they would like to receive warnings, and whether they found the media dependable and easily understandable.

\begin{tabular}{lccc}
\hline \multicolumn{1}{c}{ Media of warning } & $\begin{array}{c}\text { Received } \\
\text { warnings from } \\
\text { (in \%) }\end{array}$ & $\begin{array}{c}\text { Preferred to } \\
\text { receive warnings } \\
\text { from (in \%) }\end{array}$ & $\begin{array}{c}\text { Found the media } \\
\text { dependable and } \\
\text { easily } \\
\text { understandable } \\
\text { (in \%) }\end{array}$ \\
\cline { 2 - 4 } & \multicolumn{3}{c}{ Multiple responses were possible } \\
\hline $\begin{array}{l}\text { Electronic media } \\
\text { (radio, TV) }\end{array}$ & 67.5 & 57.0 & 53.0 \\
\hline Mega phone & 78.9 & 56.5 & 59.0 \\
\hline From other persons & 22.2 & 1.0 & 1.0 \\
\hline Signal flag & 14.4 & 4.5 & 6.0 \\
\hline Newspaper & 2.0 & 1.0 & 1.0 \\
\hline Total response & 185.0 & 120.0 & 120.0 \\
\hline
\end{tabular}

During Sidr, receiving of the warning messages were significantly related to the respondents' gender $(p=.012$ and $F=6.459)$ and occupation $(p=.003$ and $F=3.395)$. However, socio-economic status had no significant influence on receiving of the warnings by the residents during Mahasen.

\subsubsection{Warning message interpretation}

The extent to which the respondents were able to understand the information contained in the prerecorded warnings and differences in information between two warnings of the same TC after listening are presented in Table 5-4. The respondents could extract most of the information contained in the warning messages correctly. However, misinterpretation rates were high for information on: "forecasted movement direction", "time of landfall", and "surge height" (marked using italic and boldface numbers in the tables) compared to the other information, such as current TC location, danger level in Table 5-4. 
Table 5-4. Correct interpretation of the warning messages by the respondents.

\begin{tabular}{|c|c|c|c|c|c|c|}
\hline \multirow{3}{*}{$\begin{array}{l}\text { Information } \\
\text { in the } \\
\text { warning } \\
\text { message }\end{array}$} & \multicolumn{3}{|c|}{$\begin{array}{c}\text { Warnings disseminated } \\
\text { during Sidr }\end{array}$} & \multicolumn{3}{|c|}{$\begin{array}{c}\text { Warnings disseminated } \\
\text { during Mahasen }\end{array}$} \\
\hline & $\begin{array}{l}\text { Special } \\
\text { bulletin } \\
\text { No. } 5 \text { on } 12 \\
\text { Nov. } 2007\end{array}$ & $\begin{array}{l}\text { Special } \\
\text { bulletin } \\
\text { No. } 25 \text { on } \\
15 \text { Nov. } \\
2007\end{array}$ & $\begin{array}{l}\text { Change in } \\
\text { information }\end{array}$ & $\begin{array}{l}\text { Special } \\
\text { bulletin } \\
\text { No. } 8 \text { on } 12 \\
\text { May } 2013\end{array}$ & $\begin{array}{l}\text { Special } \\
\text { bulletin } \\
\text { No. } 30 \text { on } \\
16 \text { May } \\
2013\end{array}$ & $\begin{array}{l}\text { Change in } \\
\text { information }\end{array}$ \\
\hline & \multicolumn{3}{|c|}{ Percentage of the respondents } & \multicolumn{3}{|c|}{ Percentage of the respondents } \\
\hline $\begin{array}{l}\text { Current TC } \\
\text { location }\end{array}$ & 95 & 96 & 84 & 94 & 96 & 87 \\
\hline $\begin{array}{l}\text { Forecasted } \\
\text { movement } \\
\text { direction }\end{array}$ & 73 & 88 & 46 & 74 & 86 & 47 \\
\hline Danger level & 97 & 99 & 95 & 96 & 100 & 95 \\
\hline $\begin{array}{l}\text { Ports/Area } \\
\text { likely to be } \\
\text { affected }\end{array}$ & 80 & 96 & 50 & 84 & 98 & 52 \\
\hline $\begin{array}{l}\text { Time of } \\
\text { landfall }\end{array}$ & 51 & 92 & 49 & 54 & 92 & 47 \\
\hline Wind speed & 89 & 98 & 83 & 89 & 99 & 83 \\
\hline Surge height & 57 & 97 & 87 & 60 & 98 & 86 \\
\hline $\begin{array}{l}\text { Safety } \\
\text { guidance for } \\
\text { fishing boats }\end{array}$ & 86 & 94 & 41 & 88 & 95 & 40 \\
\hline
\end{tabular}

\subsubsection{Response to evacuation orders}

In the study area, $47.5 \%$ of the respondents made an evacuation after receiving the evacuation orders during Sidr and this number increased to $60 \%$ during Mahasen. Up to the year 2010, a total of 62 cyclone shelters have been constructed in the study area (CDMP, 2010; DDM (Department of Disaster Management), 2013). Currently, 57.5\% of the respondents are living within $1 \mathrm{~km}$ of a shelter.

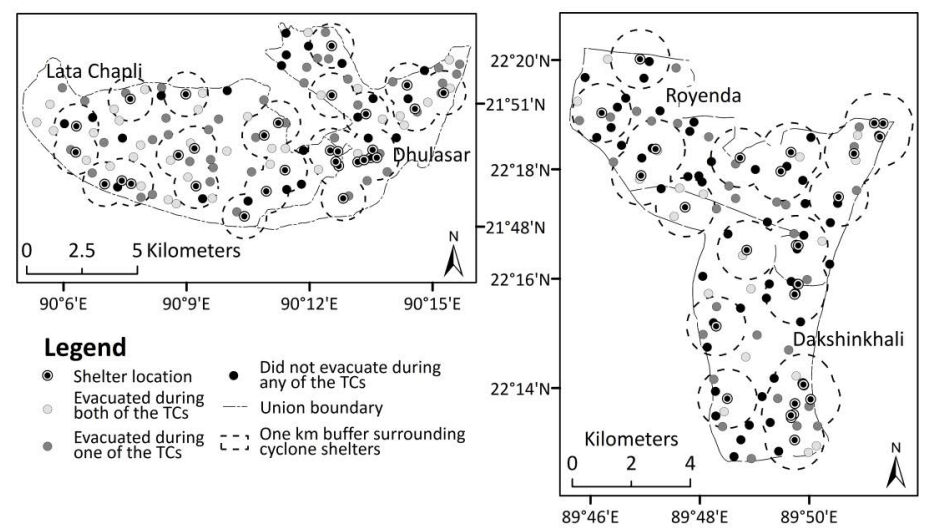

Figure 5-3. Respondents' evacuation pattern during the studied TCs. 
However, $31 \%$ of the respondents did not follow the evacuation orders during the two studied TCs and another $25 \%$ did not follow the evacuation orders during either of the TCs (Figure 5-3). The respondents who did not receive any warnings (9.5\% during Sidr and 4\% during Mahasen) are also among the respondents who did not make an evacuation during the TCs.

Respondents, who made an evacuation during the studied TCs were mostly from middlesized families (families having 4-6 family members) and lived in katcha houses. Of the evacuated respondents during Sidr, 75\% were from middle-sized families and $92 \%$ lived in katcha houses, whereas during Mahasen, $68 \%$ of the evacuated respondents were from middle-sized families and $91 \%$ used to live in katcha houses.

\subsubsection{Key reasons for non-evacuation}

To identify the key reasons for non-evacuation among the respondents and list these reasons according to their relative importance, a PCA (Principal Component Analysis) was performed on the non-evacuation reasons expressed by the respondents during the survey. The first five complex components (where component 1 is the most important and 5 is the least important) obtained through this analysis account for $68.981 \%$ of the variance of nonevacuation during Sidr (Table 5-5). The main reasons for non-evacuation were:

1. Early warning unreliable, later difficult to go far-aggregating the original variables

- Mistrust in the warning messages

- Distance to the cyclone shelter

2. Unclear warning message, later on shelter is full-aggregating the original variables

- Not understanding the instructions in the warning message

- Insufficient capacity of the nearest shelter

3. Need to protect property, while minimizing risk-aggregating the original variables

- Poor shelter condition

- Being busy sending family members to shelter and household head staying at home to save the property

4. Feeling safe at home-aggregating the original variables

- Warning message indicates that, the TC will not strike the home area

- The home being sufficiently robust

5. Insecurity-aggregating the original variable

- Being afraid of residential burglary

The first four complex components account for $67.431 \%$ of the variance of non-evacuation during Mahasen (Table 5-5) and the components were:

1. Early warning unreliable, later difficult to go far-aggregating the original variables

- Mistrust in warning message

- Poor road-transport

- Distance to the cyclone shelter 
2. Unclear warning message-aggregating the original variable

- Not understanding the instructions in the warning message

3. Need to protect property, while minimizing risk-aggregating the original variables

- Sending family members to shelter, family head staying at home to save the property, guessed that intensity of the TC will not be strong by looking at the surrounding situation

4. Feeling safe at home-aggregating the original variable

- Warning message indicates that the TC will not strike the home area

Table 5-5. Components representing the causes of non-evacuation among the respondents in the study area during the two studies TCs (extracted using PCA).

\begin{tabular}{cccc}
\hline Component & Eigenvalue & $\begin{array}{c}\text { \%o of } \\
\text { Variance }\end{array}$ & $\begin{array}{c}\text { Cumulative } \\
\text { Variance \% }\end{array}$ \\
\hline \multicolumn{4}{c}{ Extraction of the components (During TC Sidr) } \\
\hline 1 & 1.809 & 18.091 & 18.091 \\
\hline 2 & 1.521 & 15.209 & 33.300 \\
\hline 3 & 1.339 & 13.389 & 46.688 \\
\hline 4 & 1.194 & 11.936 & 58.625 \\
\hline 5 & 1.036 & 10.356 & 68.981 \\
\hline Extraction of the components (During TC Mahasen) \\
\hline 1 & 1.915 & 21.279 & 21.279 \\
\hline 2 & 1.694 & 18.826 & 40.105 \\
\hline 3 & 1.384 & 15.374 & 55.478 \\
\hline 4 & 1.076 & 11.953 & 67.431 \\
\hline
\end{tabular}

Note: Eigenvalues greater $\geq 1$ constitute a component, while components having lower eigenvalues are excluded from the analysis. This entails that the cumulative variance explained by the principal components is less than $100 \%$.

\subsubsection{Satisfaction with the warnings and suggestion for improvement}

The respondents were mostly satisfied with the disseminated warnings during the two TCs (Figure 5-4). As for the reasons for being satisfied with the disseminated warnings, $83 \%$ of the respondents claimed the warnings understandable, $71 \%$ found it timely, $60 \%$ of them agreed the warnings to be dependable, and $47 \%$ mentioned they do not have any other option for getting information about the approaching TC in an emergency situation (multiple responses were possible).

During the survey, $80 \%$ of the respondents expressed their opinion for future improvement of warning messages. They also made a number of suggestions for this improvement (multiple responses were possible):

1. $71 \%$ recommended the inclusion of a better description of the threat, such as the certainty of a TC making landfall, how urgently the residents should take protective action, and the duration of the intense cyclonic wind.

2. $48 \%$ recommended the inclusion of guidance on where to get further information about the approaching TC. 
3. $27 \%$ recommended the inclusion of guidance on protective action, such as what the residents should do to protect themselves from a TC.

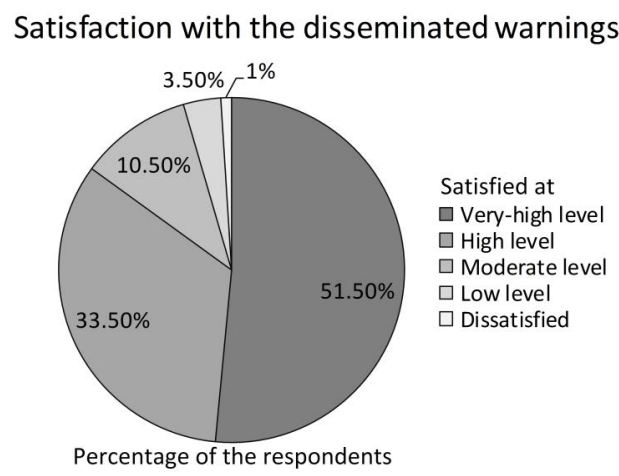

Figure 5-4. Respondents' satisfaction with the disseminated warnings.

\subsection{Results obtained during systematic parameter testing}

Better train and generalization performance was associated with bigger RFs during the test (Figure 5-5). On the basis of the result it seems logical to assume, the size of the RF must be adjusted according to the features' distribution in the input images. With smaller RFs, peripheral receiving groups in the first hidden layer could not see any meaningful part of the input (see Table 4-6). At the same time, units in these groups were forced by the kWinners-Take-All (kWTA) mechanism to produce activation. Thus, instead of mediating useful information in the feed-forward direction, units in these groups could be driven by feedback signals and bias, which might hamper learning and generalization performance.

Receptive field size $16 \times 16$

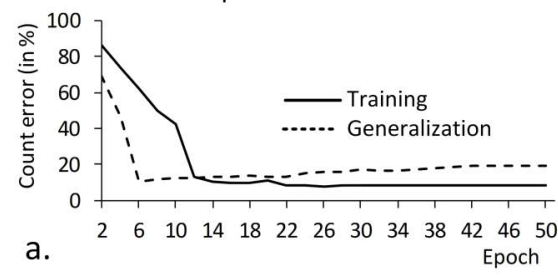

Receptive field size $26 \times 26$

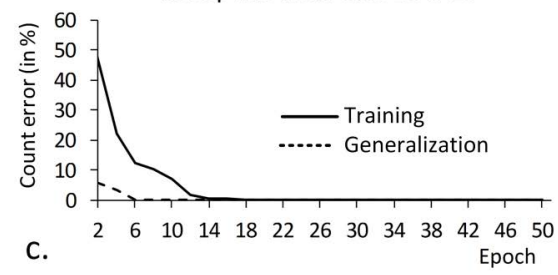

Receptive field size $20 \times 20$

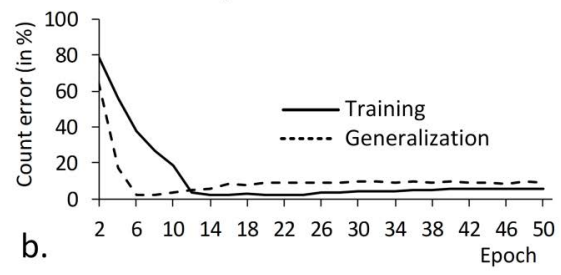

Figure 5-5. Learning and generalization performance, when RFs of a. $16 \times 16$ pixels; $\mathbf{b}$. 20 × 20 pixels; and c. $26 \times 26$ pixels in size were used for testing. 
The four networks used in this research for TC track and intensity forecasting performed better, when RFs of $32 \times 32$ pixels in size were used for the units in the first hidden layer. As the pixel values of the sea-level pressure images were not contrasting due to the coarse spatial resolution and as high wind speed values always appeared at the center leaving the peripheral parts with almost no information (see Figure 4-6), small RFs would result in the extraction of irrelevant features and could interrupt the learning of the four networks.
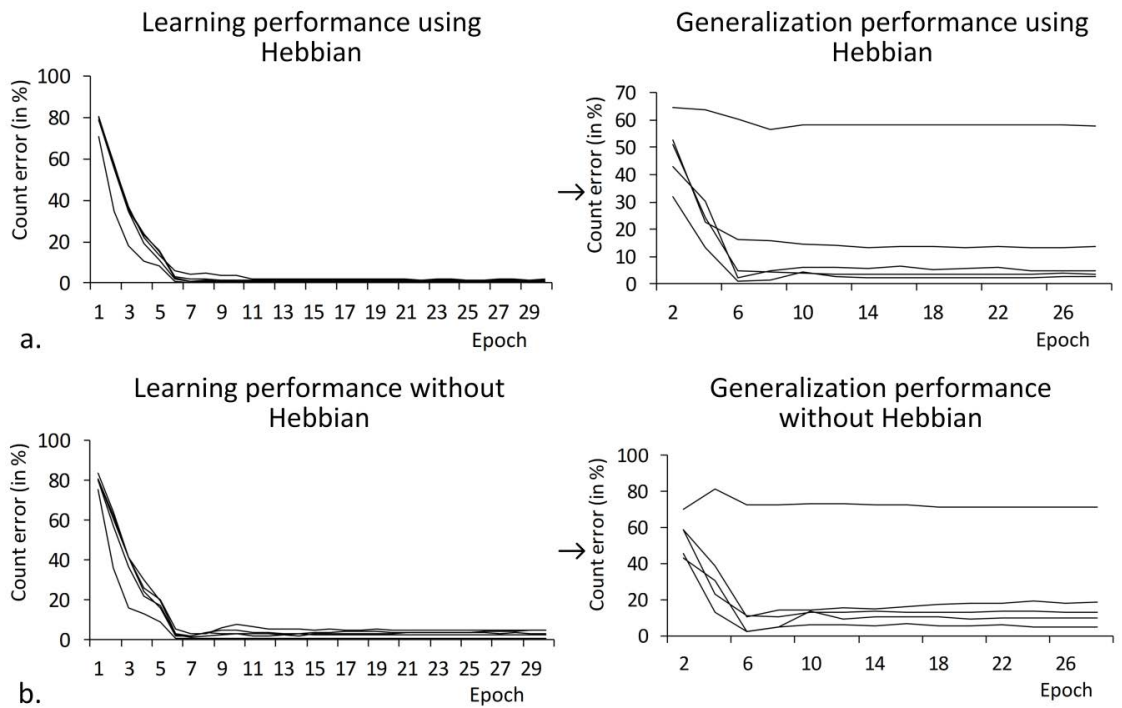

Figure 5-6. Learning and generalization performance, when the four networks were tested a. using Hebbian and b. without Hebbian.

Hebbian learning has beneficiary effects on network's performance but this does not mean that a higher rate of Hebbian learning would always correspond to better network performance. The four networks' learning and generalization performance was reasonable when only error driven learning was used. However, there was an improvement in both learning and generalization performance when a combination of Hebbian and error driven learning was used (Figure 5-6). Using a mixture of 0.001\% Hebbian, and otherwise errordriven learning at all the projections were found to be the best for the four networks used for forecasting TC track and intensity in this research.

\subsection{TC intensity forecasting performance}

The network without context layer produced intensity forecasts with between $92 \%$ and $100 \%$ accuracy, when the late-phase images (first type of test dataset) were used for testing (Figure 5-7). The network performed increasingly better, when it was tested for producing forecasts for longer time periods, for example, performed better for 24-hour forecast compared to 6-hour forecast. When the same network was tested for the images of a completely novel TC (second type of test dataset), prediction accuracy was between 30\% and $38 \%$.

Intensity prediction accuracy of the network using context layer was between $91 \%$ and $96 \%$, when the network was tested for the late-phase images (first type of test dataset) 
(Figure 5-8). Same as the network without context layers, the network using context layers produced better accuracy forecasts for longer time periods. Intensity prediction accuracy was between $32 \%$ and $45 \%$, when the network was tested for the images of a completely novel TC (second type of test dataset) (Figure 5-8). The network without context layers performed in line with the network using context layer, when they were tested for the first type of test datasets. However, the network using context layers performed better compared to the network without context layer, when the images of a completely novel TC were used for testing.

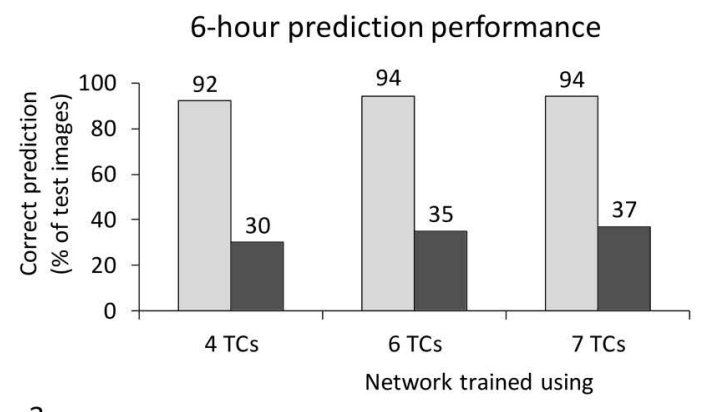

a.

$\square$ Late-phase image $\quad$ Novel TC image

12-hour prediction performance

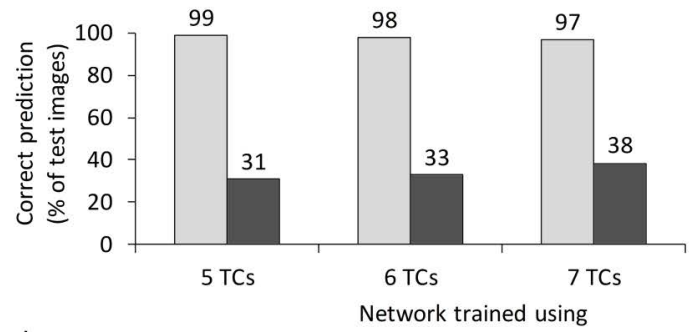

b.

$\square$ Late-phase image $\quad$ Novel TC image

24-hour prediction performance

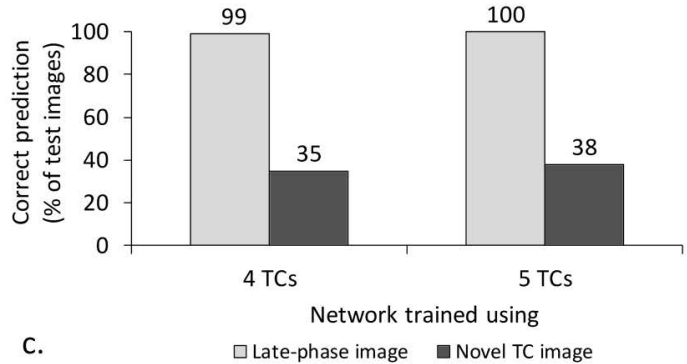

Figure 5-7. Intensity prediction performance of the network without context layers for a. 6-hour, b. 12-hour, and c. 24-hour forecast test datasets.

Interestingly, both of the intensity-forecasting networks performed progressively better for a completely novel TC, as the number of past TCs in the training set was increased (Figure 
5-7 and Figure 5-8). This indicates the necessity of a large training dataset for producing good accuracy forecasts for novel TCs.

6-hour prediction performance

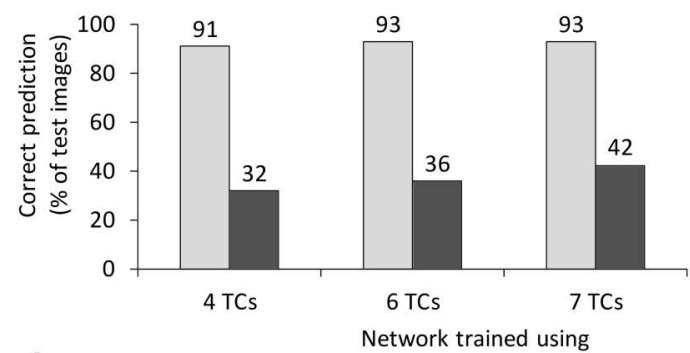

a.

$\square$ Late-phase image $\quad$ Novel TC image

12-hour prediction performance

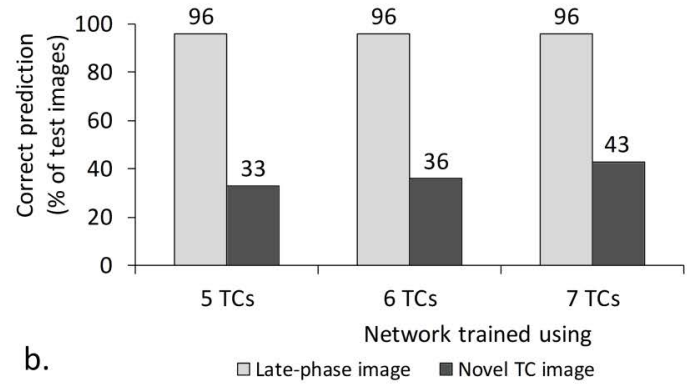

24-hour prediction performance

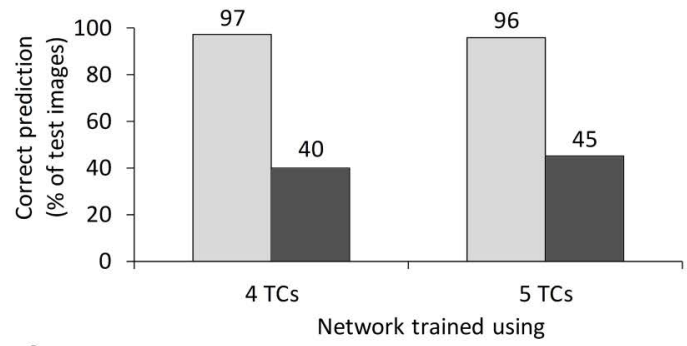

C. $\quad$ Late-phase image $\quad$ Novel TC image

Figure 5-8. Intensity prediction performance of the network using context layers for a. 6-hour, b. 12-hour, and c. 24-hour forecast test datasets.

\subsubsection{Pattern of intensity forecasting error}

The types of errors produced by the two intensity-forecasting networks during testing were similar. As the two networks produced very few errors for the 24-hour forecast test datasets (Figure 5-7 and Figure 5-8), this result was not used for generating error pattern plots. 
Analyzing the errors, both of the intensity-forecasting networks produced around $95 \%$ oneintensity-category-off and 5\% two-intensity-category-off errors (Figure 5-9a), when they were tested for the 6-hour forecast test datasets consisting of late-phase images of seven TCs. For the 6-hour forecast test dataset of a novel TC (images of hurricane Bill), the network without context layers produced $60 \%$ one-intensity-category-off, $18 \%$ twointensity-category-off, $15 \%$ three-intensity-category-off, and $7 \%$ four-intensity-category-off errors (Figure 5-9b). The proportions of one, two, three, and four intensity-category-off errors produced by the network using context layers for the 6-hour dataset a novel TC were $69 \%, 15 \%, 10 \%$, and $6 \%$ respectively (Figure $5-9 b$ ).

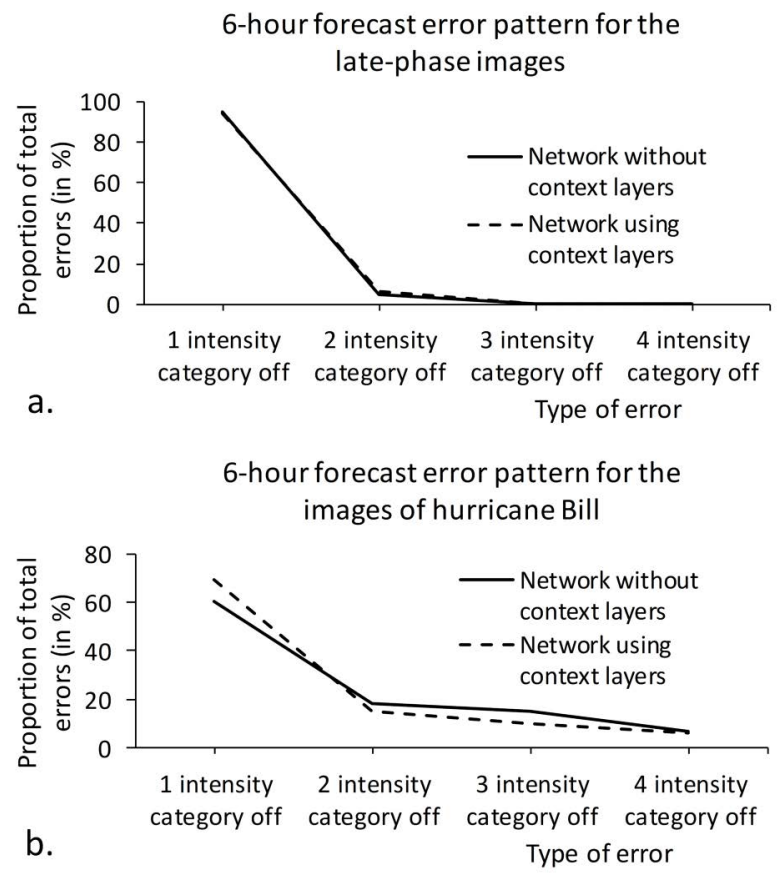

Figure 5-9. Illustrate error patterns, when 6-hour forecast test datasets were presented to the two intensity-forecasting networks: a. error patterns, when late-phase images of seven TCs were presented, and $\mathbf{b}$. error patterns, when images of hurricane Bill were presented.

All the errors produced by both of the intensity-forecasting networks were one-intensitycategory-off, when the 12-hour forecast test datasets consisting of late-phase images of seven TCs were used for testing (Figure 5-10a). Of the total errors produced by the network without context layers for the 12-hour forecast test images of a novel TC, $68 \%$ were oneintensity-category-off, 20\% two-intensity-category-off, $6 \%$ three-intensity-category-off, and $6 \%$ were four-intensity-category-off (Figure 5-10b).

The network using context layers performed better for the 12-hour forecast test images of a novel TC, where $74 \%$ of the total errors were one-intensity-category-off, $15 \%$ twointensity-category-off, 5\% three-intensity-category-off, and $6 \%$ were four-intensitycategory-off (Figure 5-10b). 
12-hour forecast error pattern for the

late-phase images

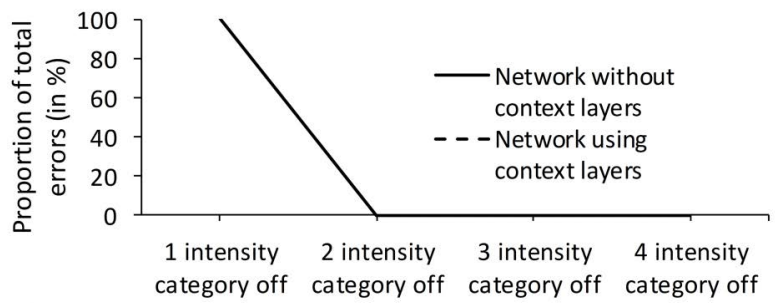

a.

Type of error

12-hour forecast error pattern for the images of hurricane Bill

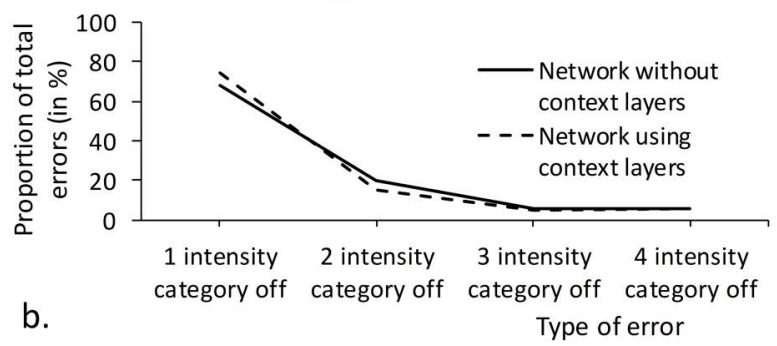

Figure 5-10. Illustrate error patterns, when 12-hour forecast test datasets were presented to the two intensity-forecasting networks: a. error patterns, when late-phase images of seven TCs were presented, and $\mathbf{b}$. error patterns, when images of hurricane Bill were presented.

\subsection{Combined TC track and intensity forecasting performance}

It has previously been mentioned that the two combined TC track and intensity forecasting networks were tested only for the 12-hour forecast test datasets consisting of late-phase images.

Prediction performance for late-phase images

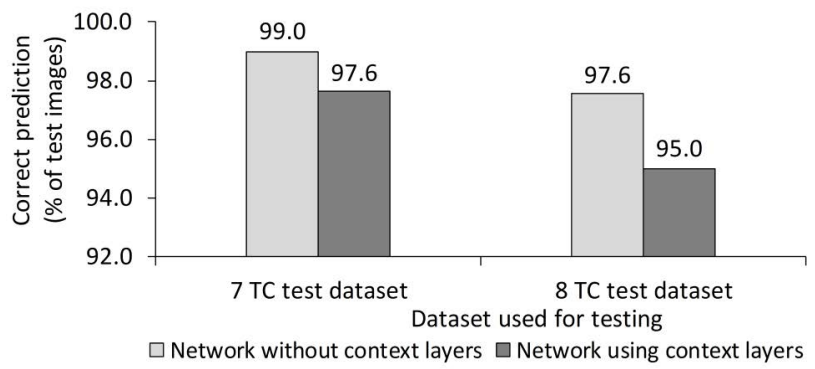

Figure 5-11. TC track and intensity prediction performance produced by the two combined track and intensity forecasting networks. 
For the late-phase images of seven TCs, the two combined track and intensity prediction networks could predict TC track and intensity levels between 98\% and 99\% accuracy. Prediction accuracy remained between $95 \%$ and 98\%, when the late-phase images of eight TCs were used for testing (Figure 5-11).

Network using context layers

prediction performance for individual output

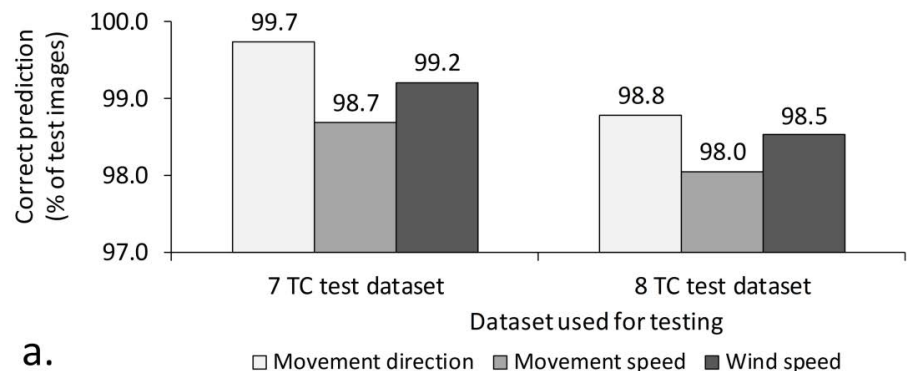

Network without context layers prediction performance for individual output

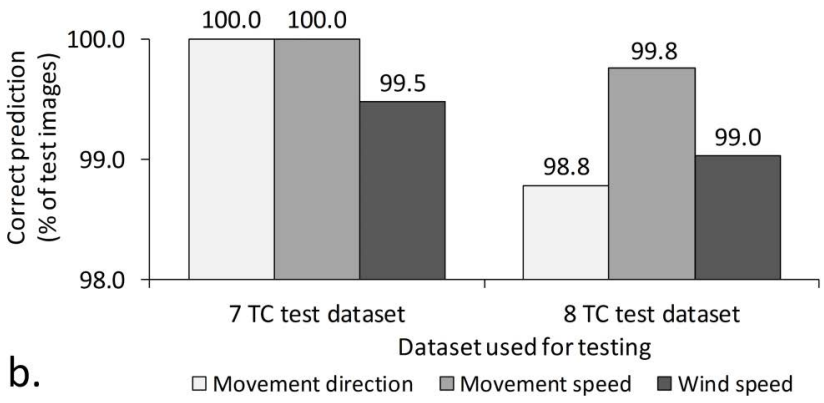

Figure 5-12. a. Illustrates prediction performance for individual outputs produced by the network using context layers, and $\mathbf{b}$. illustrates prediction performance for individual outputs, when context layers were not used.

To produce correct track and intensity forecasts for a set of test image presented to the two networks, the network-generated prediction was required to be correct for all the three outputs: TC movement direction, TC movement speed, and wind speed. Therefore, the overall prediction performance (Figure 5-11) did not correspond to the two networks' prediction performance for individual outputs (Figure 5-12a and b). 


\section{Chapter 6}

\section{Discussion}

The two questions that this thesis has set out to answer was understanding the problems associated with the current cyclone early warning system in Bangladesh and investigating the feasibility of biologically based ANNs in producing TC track and intensity forecasts.

\subsection{Problems associated with cyclone early warning in Bangladesh}

The results of the survey among residents in the coastal areas reveal that a number of reasons, in particular uninformative and unreliable warnings, can cause hesitation and delay the decision for evacuation among the residents. This prevents the residents in the coastal areas from following the evacuation orders during the two studied TCs. Previous research evaluating residents' response to evacuation orders in the coastal areas of Bangladesh also identified uninformative and unreliable warnings as one of the main reasons for non-evacuation among the residents in the coastal areas of Bangladesh (Mallick et al., 2009; A. Paul \& Rahman, 2006; B. K. Paul, 2012; B. K. Paul \& Dutt, 2010). However, due to a receiver-centric evaluation of the cyclone early warning system, previous research failed to describe why the warning messages are uninformative at the provider's end. As this thesis additionally elicited meteorologists' views when evaluating the cyclone early warning system, it was possible to identify the problems associated with $\mathrm{TC}$ forecasting at BMD. Results of the interview among the meteorologists indicate that data scarcity over the BoB and limitations associated with the operational TC forecasting technique itself are the main reasons for disseminating inaccurate and uninformative warning messages by BMD.

BMD has access to ocean buoy and atmospheric reanalysis data (Kalnay et al., 1996; Simmons et al., 2007) and these data could be used for solving data scarcity issues over the $\mathrm{BoB}$. As the operational TC forecasting technique does not support these data-file formats, meteorologists cannot use them for improving TC forecasting accuracy. Numerical techniques currently in operation at BMD used for general weather forecasting would support these data-file formats; however, these techniques have originally been developed for forecasting rainfall, temperature, and TC-induced storm surge levels. The meteorologists do not have the necessary skills and computational knowledge to modify these numerical techniques to adapt them for forecasting TCs.

\subsection{TC track and intensity forecasting using biologically based ANNs}

The application of three-layer feed forward networks is well known in TC track and intensity prediction (Ali et al., 2007; Jin et al., 2008; Pickle, 1991). However, these 
techniques lack the capability of spatial feature extraction; instead they use approaches for recognizing patterns that is similar to the "bag-of-features" method ( $\mathrm{Li} \&$ Itti, 2011; Nowak, Jurie, \& Triggs, 2006; O'Hara \& Draper, 2011). As a result, utilizing the spatial relationships of the features present in the $2 \mathrm{D}$ input images, which are closely associated with TC track and intensity change, seems difficult using the standard three-layer networks. The TC track and intensity forecasting technique developed in this research instead uses deep biologically based ANNs for producing forecasts on the basis of multi-instrument 2D images. These biologically based ANNs are characterized by the following interesting properties:

- Recurrent connections - this type of connection between neighboring layers allows for simultaneous bidirectional (bottom-up and top-down) information exchange, which means that processing can be optimized in parallel towards multiple constraints.

- Combined supervised and unsupervised learning, achieved through local, unit-wise combination of bottom-up activations and top-down error signals. In this way, unsupervised learning of low-level features can be geared towards the task requirements through error signals.

- Deep, hierarchical network architecture, where absolute spatial position and feature size is abstracted away step-by-step at multiple processing levels. This allows for position- and size-independent pattern recognition, which is normally considered a computationally demanding problem involving many-to-many mapping.

With these characteristics, it seems logical to assume that the developed TC track and intensity forecasting technique can solve the technical problems associated with TC forecasting at $\mathrm{BMD}$, and is likely to contribute to an improved cyclone early warning system in Bangladesh.

\subsubsection{TC Intensity forecasting}

The two intensity-forecasting networks could produce correct intensity prediction for more than $90 \%$ of the first type of test dataset (consisting of images recorded during the end of the TC lifecycles). The same two networks could predict the intensity levels with between $30 \%$ and $45 \%$ accuracy when the second type of test images (images of a completely novel hurricane Bill) were used for testing. This intensity prediction performance produced by the two networks indicates that biologically based ANNs can be practically useful for producing TC intensity forecasts.

Wrong interpretation of the intensity levels by the two networks was mainly associated with cases when the patterns in the five input images did not change in relation to the observed intensity categories in the test dataset or vice versa. The low-resolution images (60 x 60 pixels) used for training and testing of the two networks do not seem to exactly reveal all the distinctive patterns visible in the original images. Conversely, seven output intensity categories represent a generalized view of the observed wind speeds during TC lifecycle in the TC best track data. As the average wind speed difference between two successive output intensity categories was $33.25 \mathrm{~km} / \mathrm{h}$, intensity change smaller than 33.25 $\mathrm{km} / \mathrm{h}$ could not be captured using the output layer with seven output intensity categories.

Changes in the observed intensity levels within a 6-hour time interval are more likely to be small-scale. Though a new set of images was available every 6 hour, the inputs might 
happen to be the same as for another intensity level due to low level of details. As a result, small-scale intensity change that occurred within a 6-hour time interval could not be forecasted correctly for all the inputs in the 6-hour forecast datasets (Figure 5-7). In contrast, in the 12- and 24-hour forecast datasets, changes in the patterns associated with the five types of input images, as well as changes in the observed intensity levels are more likely to be large-scale, which could be better elicited using the low-resolution $(60 \times 60$ pixels) images. Therefore, the two intensity-forecasting networks could produce better accuracy forecasts for the 12- and 24-hour forecast datasets, than for the 6-hour forecast datasets during testing (Figure 5-7 and Figure 5-8).

\section{Comparison of intensity prediction performance}

To compare the network-generated intensity prediction error with the intensity prediction error of the operational forecasting techniques in the Atlantic basin, the two intensityforecasting networks' 12 -hour prediction errors for late-phase images, and for a completely novel TC, were converted into $\mathrm{km} / \mathrm{h}$ errors in two steps. First, the binary distance between the network-activated output unit and the correct intensity unit was measured for each image presented to the two networks during testing. Then these unit distances (error types) were multiplied by $33.25 \mathrm{~km} / \mathrm{h}$, which is the wind speed difference between two neighboring units (TC intensity categories) in the output layer in the two networks (Figure $6-1 a$ and $b)$.

\section{Distribution of 12-hour intensity prediction error network using context layers}

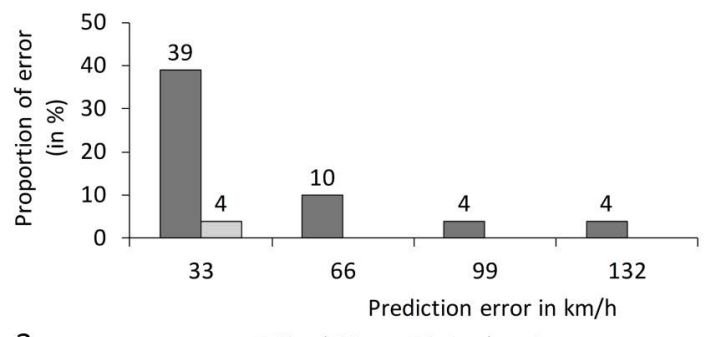

a. $\square$ Novel TC $\square$ Late-phase images

\section{Distribution of 12-hour intensity prediction error} network without context layers

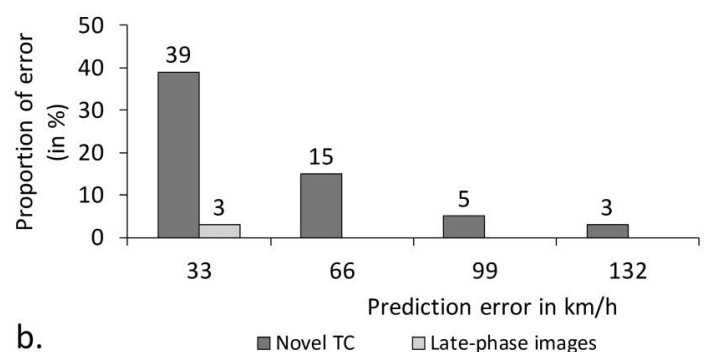

Figure 6-1. 12-hour intensity prediction error (in $\mathrm{km} / \mathrm{h}$ ) produced by: a. the network using context layers, and $\mathbf{b}$. the network without context layers. 
According to these analyses, the two networks' intensity prediction performance for the 12 hour test datasets consisting of late-phase images seems better compared to the intensity prediction performance of the currently operational TC intensity forecasting techniques in the Atlantic basin. The intensity prediction performance ( 12 hours ahead) of the operational TC forecasting techniques specialized for the Atlantic basin, such as the Decay Statistical Hurricane Intensity Forecast (DSHIFOR5), the Statistical Hurricane Intensity Prediction Scheme (SHIPS), the Geophysical Fluid Dynamics Laboratory $(G F D L)$, and the Hurricane Weather Research and Forecast $(H W R F)$ is visualized in Figure 6-2 (Franklin, 2009). Compared to this intensity prediction performance, the network without context layers produced only $0.99 \mathrm{~km} / \mathrm{h}$ mean error, when it was tested for the $12-$ hour forecast late-phase images (Figure 6-2). When the network was tested for a novel TC, the intensity prediction error was $31.7 \mathrm{~km} / \mathrm{h}$ (Figure 6-2). The network produced this result when it was trained using low-resolution 2D images of only seven TCs formed in the Atlantic basin during 2008, and was tested for a completely new TC, formed in the same basin, but in a different year, in 2009 .

Mean 12-hour intensity prediction error

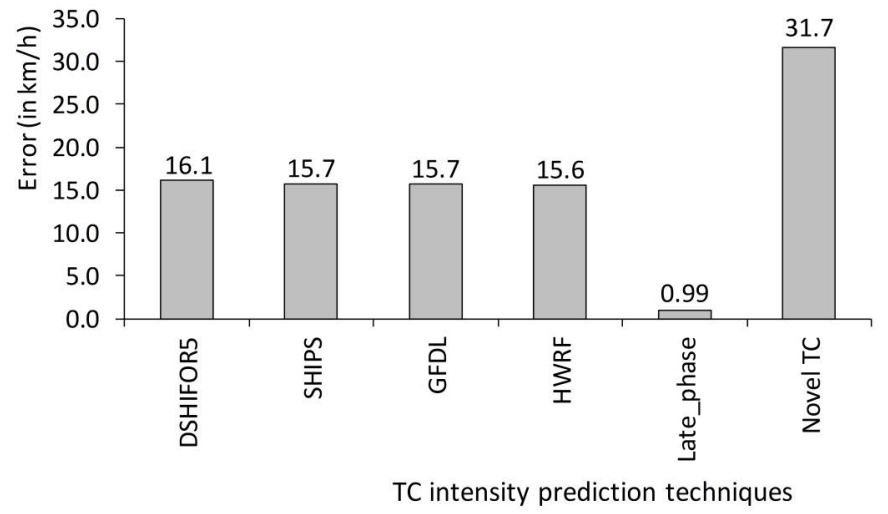

Figure 6-2. Comparison of the mean intensity prediction errors produced by various intensity prediction techniques in the Atlantic basin in 2008 (Franklin, 2009) and the $12-$ hour mean intensity prediction error produced by the network without context layers for the late-phase images, and for a novel TC in the same basin.

Producing accurate intensity forecast is usually more important towards the end of a TC's lifecycle (when it is about to cross the coast) than in the beginning, when the TC has just formed. On the basis of the intensity prediction performance for the late-phase images (Figure 6-2), it seems reasonable to assume that the technique is effective in forecasting TCs that are approaching land.

Moreover, the two intensity-forecasting networks seem more effective than existing ANN-based TC intensity forecasting techniques. The TC identification and tracking technique developed by Lee and Liu (2000) produces TC intensity forecasts on the basis of three-hourly visible and infrared channel satellite images of 120 TCs over the Atlantic basin that occurred between 1985 and 1998. Of the total number of images, 600 randomly chosen TC images ( 5 images for each TC) were used for testing. Both visible and infrared channel satellite images were used for testing. The correct intensity prediction rates achieved for these two types of test images were $82 \%$ and $95 \%$, respectively (Lee \& Liu, 2000). Another 
ANN-based TC intensity forecasting technique, the SElection MOdule and MAtching MOdule (SEMO-MAMO), developed by Feng and Liu (2004), using a modified Hausdorff distance, produced TC intensity forecasts by matching the TC image contours with Dvorak template contours. On an average, this technique produces forecasts with $74.32 \%$ accuracy (Feng \& Liu, 2004). As opposed to intensity prediction performance of the existing ANNbased techniques, the two intensity-forecasting networks could correctly forecast intensity levels for $91-94 \%$ of the cases, when the 6-hour test datasets of late-phase images were used for testing. TC intensity prediction was correct for $96-100 \%$ of the cases, when the same two networks were tested for the 12-, and 24-hour datasets consisting of late-phase images (see section 5.4).

\subsubsection{Graphically-presented TC track and intensity forecasting-ongoing work}

Many of the operational TC forecasting techniques, such as the Geophysical Fluid Dynamics Laboratory (GFDL), the Hurricane Weather Research and Forecasting Model (HWRF), the United Kingdom Met Office model (UKMET), and the FSU Super-ensemble (FSSE), are capable of producing forecasts for both track and intensity (Cangialosi \& Franklin, 2014; Franklin, 2009; Lean et al., 2008). This capability can be useful for the technique developed in this research in two ways:

1. First, training time could be minimized, as training of a single network would be enough for producing track and intensity forecasts.

2. Second, the produced track and intensity forecasts could be managed more easily for visualization (presentation to the end-user), as both of the tasks (track and intensity forecasting) would be performed by a single network.
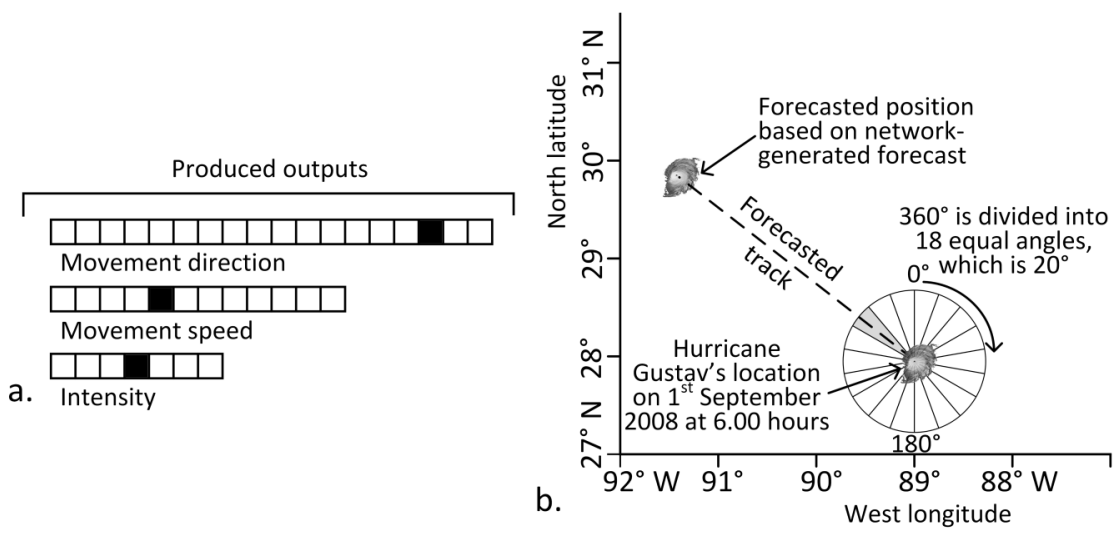

Figure 6-3. TC track and intensity prediction using network-generated outputs. a. Outputs produced by the two networks. b. Forecasted movement direction (filled using grey color) and position. Dotted line in this figure illustrates the distance between the current and forecasted TC locations as well as the forecasted track for the next 12 hours.

The two combined TC track and intensity forecasting networks used three output layers: intensity, movement speed, and movement direction to produce forecasts for TC track and intensity at the same time. TC intensity forecasts were produced into seven intensity categories, which is the same as the two intensity-forecasting networks. Movement speed 
forecasts were produced in 12 categories, where $1 \mathrm{~km} / \mathrm{h}$ and $60 \mathrm{~km} / \mathrm{h}$ were considered as the lowest, and the highest TC movement speeds respectively, this range covers the movement speeds from an almost stationary TC to a fast-moving TC. The leftmost unit in the movement speed layer represented movement speed of $\leq 5 \mathrm{~km} / \mathrm{h}$ and the rightmost unit represented movement speed of $60 \mathrm{~km} / \mathrm{h}$. Therefore, a movement speed difference between two neighboring units was $5 \mathrm{~km} / \mathrm{h}$. TC movement direction forecasts were produced in 18 categories $\left(360^{\circ}\right.$ was divided into 18 equal angles). As a result, the networks could consider variations in $\mathrm{TC}$ movement direction in $20^{\circ}$ increments (Figure 6-3).

TC track and intensity outputs produced by the two combined track and intensity forecasting networks for the image of hurricane Gustav (see Table 4-7 in section 4.3.4) recorded at 6.00 hours on the first of September 2008 are visualized in Figure 6-3a. According to the TC best track data, at this time TC Gustav's location was $27.9^{\circ} \mathrm{N}$ latitude and $89.0^{\circ} \mathrm{W}$ longitude. To get Gustav's forecasted location after 12 hours, the networkgenerated forecast can be interpreted as:

1. Gustav is expected to move in a direction between $300^{\circ}$ and $320^{\circ}$ (Figure 6-3b), as the two networks activated the third unit from the right in the movement direction layer.

2. Hurricane Gustav may move around 300 kilometers $^{5}$ in a $310^{\circ}$ direction (considering the mid-point of $300^{\circ}$ and $320^{\circ}$ ) during the next 12 hours, as both of the networks activated the fifth unit from the left in the movement speed layer. Therefore, after 12 hours hurricane Gustav's most likely position would be $29.7^{\circ} \mathrm{N}$ and $91.3^{\circ} \mathrm{W}$, which is 300 kilometers away in the $310^{\circ}$ direction from the current position $\left(27.9^{\circ} \mathrm{N}\right.$ and $\left.89.0^{\circ} \mathrm{W}\right)$. The observed position of hurricane Gustav at 18.00 hours on the first of September 2008 was $29.8^{\circ} \mathrm{N}$ and $91.4^{\circ} \mathrm{W}$, which is only 15 kilometers away to the northwest of the forecasted position.

3. After 12 hours, hurricane Gustav is most likely to be a "category two" TC, as the two networks activated the fourth unit from the left in the intensity layer, which notably was a correct intensity prediction.

\subsubsection{Prediction performance improvement}

The four networks' track and intensity prediction performance for the late-phase images of already seen TCs, as well as performance for completely novel TC is promising. How additional improvements would contribute to the networks' prediction performance, particularly for the images of a novel TC remains to be investigated:

1. The two intensity-forecasting networks performed increasingly better for a novel TC, when they were trained using images of more historical TCs. This improvement indicates that using images of a large number of historical TCs for training are important for enhancing the networks' performance during testing.

\footnotetext{
5 The network-activated unit (the 5 th unit from the left) in the movement speed layer was multiplied by the movement speed difference between two neighboring units $(5 \times 5=25 \mathrm{~km} / \mathrm{h})$. Then, 25 $\mathrm{km} / \mathrm{h}$ was multiplied by the time interval between the two successive images (which was 12 hours) to obtain the distance hurricane Gustav is expected to move during the next 12 hours.
} 
2. The two intensity-forecasting networks produced forecasts into seven intensity categories, whereas the two combined track and intensity forecasting networks produced track and intensity forecasts into eighteen movement directions and seven intensity categories respectively. This resulted in an output resolution of $20^{\circ}$ directional steps, and $33.25 \mathrm{~km} / \mathrm{h}$ wind speed steps between two neighboring output units. However, it seems reasonable to assume that for producing forecasts with a higher-resolution of movement direction and intensity, the four networks also need to be trained and tested using higher resolution images.

3. Additional types of data, for example, velocity of vertical wind could be used to include the distribution of heat energy in the vortex of a TC. Relative humidity could be used to measure whether sufficient moisture is available for convection to take place and increase the moist static energy for TC intensification. The availability of moisture in the boundary layer also has influence on TC track, therefore using this information as predictor could also increase TC track prediction accuracy (Chan \& Kepert, 2010). Terrain information could be useful to include, the effect of land and mountains on TC track and intensity.

4. The European Centre for Medium-Range Weather Forecasts (ECMWF) reanalysis data (Simmons et al., 2007) is available in higher spatial resolution (each pixel covers an area of $0.125^{\circ}$ latitude x $0.125^{\circ}$ longitude) compared to NCEP (national center for environmental prediction) reanalysis data, where each pixel covers an area of $2.5^{\circ}$ latitude $\times 2.5^{\circ}$ longitude. Therefore, ECMWF data could be used to enhance TC track and intensity prediction accuracy produced by the four networks.

5. The vortex of an intense TC extends high in the atmosphere. Therefore, using data from various pressure levels could provide valuable information about TC track and intensity change and thus could increase the prediction performance of the four networks.

6. The four networks were simulated on a $2.4 \mathrm{GHz}$ quad-core PC using 3 gigabytes of RAM. Though the use of low-resolution images made the simulation possible on a low profile $\mathrm{PC}$, much valuable information contained in the original images might have been lost. Computers that are more powerful could be used to train and test upscaled versions of the four networks on the basis of higher-resolution images for producing TC track and intensity forecast. 


\section{Chapter 7}

\section{Conclusions and future work}

The primary goal of this research was to contribute to improved cyclone early warning systems in countries with less economic and technical means. This research identified problems associated with the reliability of cyclone early warnings in Bangladesh. The early cyclone warnings that are formulated by BMD (Bangladesh Meteorological Department) are often unreliable, are difficult to interpret, and contain insufficient information about the approaching TC. Part of the problems can be attributed to ineffective TC forecasting at $\mathrm{BMD}$, which in turn is a result of data scarcity over the BoB (Bay of Bengal), inadequate computational resources, insufficient skills and computational expertise of the meteorologists at BMD, and that currently inappropriate forecasting techniques are used.

Although producing accurate TC track and intensity forecasts is very important for effective early warning, BMD's TC forecasting quality has not received sufficient attention in earlier research (Alam \& Collins, 2010; U. Haque et al., 2012; B. K. Paul, 2012), neither has it been addressed in the national cyclone preparedness plan (DDM (Department of Disaster Management), 2013). In general, the current initiatives taken by the Bangladesh government and by non-government organizations for improving the efficiency of the cyclone early warning system seem inadequate for tackling BMD's TC forecasting ability, even though further improvements are necessary (DDM (Department of Disaster Management), 2009, 2013; DMB (Disaster Management Bureau), 2010; The World Bank, 2013).

To address the problems associated with TC forecasting at BMD, a technique using biologically based ANNs has been explored with the following characteristics:

- Low requirements on meteorological data, as only remotely sensed data is used as input, as opposed to other forms of meteorological data collected using groundbased measuring stations and weather balloons.

- Low requirements on computational resources - all four networks used for TC track and intensity forecasting in this thesis were trained and tested on an ordinary desktop PC.

- Performs on par with the operational TC forecasting techniques in the Atlantic basin (Franklin, 2009).

- Can be applied to arbitrary basins by using training data from that basin.

- Can produce more accurate TC intensity forecasts than existing ANN-based techniques (Feng \& Liu, 2004; Lee \& Liu, 2000). 
With these capabilities, the ANN technique developed in this research can ultimately contribute to improve the efficiency of the current cyclone early warning system in countries, such as Bangladesh.

The prediction performance of the developed track and intensity forecasting technique is promising. However, the technique is new and still under development. Before the technique can become operational, several issues need to be further addressed:

1. Scaling up the networks to be able to use original satellite images (not downsized) for training and testing.

2. Extending the networks to handle TCs with irregular motion pattern, such as sudden recurving or looping, as well as TCs with irregular intensity patterns, such as rapid intensifying or weakening.

3. When a TC approaches the coast, the winds within the TC interact with land. Also, the moisture supply from the warm ocean waters is reduced close to land, which could have considerable influence on both TC track and intensity development before a landfall (Chan \& Kepert, 2010). An image containing information about the landmass surrounding the ocean basin could be used as input to the networks to handle this situation.

4. Designing a graphical user interface to visualize the forecasted track and intensity levels on a map. 


\section{Chapter 8}

\section{References}

Aberson, S. D. (1998). Five-Day Tropical Cyclone Track Forecasts in the North Atlantic Basin. Wea. Forecasting, 13(4), 1005-1015. http://doi.org/10.1175/15200434(1998)013<1005:FDTCTF>2.0.CO;2

Aberson, S. D., Cione, J., Wu, C. C., Bell, M. M., Halverson, J., Fogarty, C., \& Weissmann, M. (2010). Aircraft Observations of Tropical Cyclones. In J. C. L. Chan \& J. D. Kepert (Eds.), Global Perspectives on Tropical Cyclones - From Science to Mitigation (pp. 227-240). World Scientific Publishing Company.

ABM (Australia Bureau of Meteorology). (2009). Warning strategies. Retrieved March 30, 2011, from http://www.cawcr.gov.au/bmrc/pubs/tcguide/ch7/ch7_3.htm

ADRC (Asian Disaster Reduction Center). (2005). Total disaster risk mānagement - good practice (pp. 32-33). Kobe, Japan.

Aguilar-Martinez, S., \& Hsieh, W. W. (2009). Forecasts of Tropical Pacific Sea Surface Temperatures by Neural Networks and Support Vector Regression. International Journal of Oceanography, International Journal of Oceanography, 2009, 13. http://doi.org/10.1155/2009/167239, 10.1155/2009/167239

Aisa, B., Mingus, B., \& O'Reilly, R. (2008). The emergent neural modeling system. Neural Networks, 21(8), 1146-1152.

Akhand, M. H. (2003). Disaster Management and Cyclone Warning System in Bangladesh. In J. Zschau \& A. Küppers (Eds.), Early Warning Systems for Natural Disaster Reduction (pp. 49-64). Springer Berlin Heidelberg. Retrieved from http://link.springer.com/chapter/10.1007/978-3-642-55903-7_8

Alam, E., \& Collins, A. E. (2010). Cyclone disaster vulnerability and response experiences in coastal Bangladesh. Disasters, 34(4), 931-954. http://doi.org/10.1111/j.14677717.2010.01176.x

Ali, M. M., Kishtawal, C. M., \& Jain, S. (2007). Predicting cyclone tracks in the north Indian Ocean: An artificial neural network approach. Geophys. Res. Lett., 34(4), L04603.

Anderson-Berry, L. J. (2003). Community vulnerability to tropical cyclones: Cairns, 19962000. Natural Hazards, 30(2), 209-232.

Asgary, A., \& Halim, A. (2011). Measuring people's preferences for cyclone vulnerability reduction measures in Bangladesh. Disaster Prevention and Management: An International Journal, 20(2), 186-198. http://doi.org/10.1108/09653561111126111

Baker, E. J. (1991). Hurricane evacuation behavior. International Journal of Mass Emergencies and Disasters, 9(2), 287-310.

Bankert, R. L., \& Tag, P. M. (2002). An Automated Method to Estimate Tropical Cyclone Intensity Using SSM/I Imagery. Journal of Applied Meteorology, 41(5), 461. 
Barkmeijer, J., Buizza, R., Palmer, T. N., Puri, K., \& Mahfouf, J.-F. (2001). Tropical singular vectors computed with linearized diabatic physics. $Q J$ Roy Meteor Soc, 127(572), 685-708. http://doi.org/10.1002/qj.49712757221

BBS (Bangladesh Bureau of Statistics). (2012). Bangladesh Population \& Housing Census 2011. Dhaka, Bangladesh.

Bedka, K. M., Velden, C. S., Petersen, R. A., Feltz, W. F., \& Mecikalski, J. R. (2009). Comparisons of Satellite-Derived Atmospheric Motion Vectors, Rawinsondes, and NOAA Wind Profiler Observations. Journal of Applied Meteorology and Climatology, 48(8), 1542-1561. http://doi.org/10.1175/2009JAMC1867.1

Bender, M. A., \& Ginis, I. (2000). Real-Case Simulations of Hurricane-Ocean Interaction Using A High-Resolution Coupled Model: Effects on Hurricane Intensity. Mon. Wea. Rev., 128(4), 917-946. http://doi.org/10.1175/15200493(2000)128<0917:RCSOHO $>2.0 . \mathrm{CO} ; 2$

Berg, H. V. D., Wetherell, M., \& Houtkoop-Steenstra, H. (Eds.). (2003). Analyzing Race Talk: Multidisciplinary Perspectives on the Research Interview. Cambridge University Press.

Bin, W., Elsberry, R. L., Yuqing, W., \& Liguang, W. (1999). Dynamics in Tropical Cyclone Motion: A Review. Chinese J Atmos Sci, 22(4), 416-434.

BMD (Bangladesh Meteorological Department). (2010). Tropical cyclone records in the Bay of Bengal.

Borji, A., \& Itti, L. (2014). Human vs. Computer in Scene and Object Recognition. In 2014 IEEE Conference on Computer Vision and Pattern Recognition (CVPR) (pp. 113120). http://doi.org/10.1109/CVPR.2014.22

Boullé, P. (1999). IDNDR. Retrieved March 29, 2011, from http://www.emforum.org/vforum/990623.htm

Boyatzis, R. E. (1998). Transforming qualitative information: Thematic analysis and code development. Sage.

Boyce, C., \& Neale, P. (2006). Conducting in-depth interviews: A guide for designing and conducting in-depth interviews for evaluation input (p. 12). Pathfinder International Watertown, MA.

Bradburn, N. M., Sudman, S., \& Wansink, B. (2004). Asking Questions: The Definitive Guide to Questionnaire Design -- For Market Research, Political Polls, and Social and Health Questionnaires. John Wiley \& Sons.

Brad, R., \& Letia, I. A. (2002). Cloud motion detection from infrared satellite images. In Second International Conference on Image and Graphics (pp. 408-412). International Society for Optics and Photonics.

Brady, B. J. (2005). Hurricane forecasting and warning system (Workshop report) (p. 166). NCAR, California.

Brand, S., \& Blelloch, J. W. (1973). Changes in the Characteristics of Typhoons Crossing the Island of Taiwan (No. NAVENVPREDRSCHFAC Technical Paper No. 8-73) (p. 21). Monterey CA: Naval Research Laboratory.

Brand, S., Buenafe, C. A., \& Hamilton, H. D. (1981). Comparison of tropical cyclone motion and environmental steering. Monthly Weather Review, 109, 908.

Braun, V., \& Clarke, V. (2006). Using thematic analysis in psychology. Qualitative Research in Psychology, 3(2), 77-101. http://doi.org/10.1191/1478088706qp063oa

Buizza, R., Milleer, M., \& Palmer, T. N. (1999). Stochastic representation of model uncertainties in the ECMWF ensemble prediction system. QJ Roy Meteor Soc, 125(560), 2887-2908. 
Camargo, S. J., \& Sobel, A. H. (2005). Western North Pacific Tropical Cyclone Intensity and ENSO. Journal of Climate, 18(15), 2996-3006.

Cangialosi, J. P., \& Franklin, J. L. (2014). 2013 National Hurricane Center Forecast Verification Report (p. 84). National Hurricane Center.

Cartwright, H. M., \& Kharma, N. (2008). Using artificial intelligence in chemistry and biology: a practical guide. CRC Press.

CCNLab. (2014). Emergent neural network simulator. Retrieved October 31, 2014, from https://grey.colorado.edu/emergent/index.php/Main_Page

CDMP, M. of D. M. and R., Bangladesh. (2010). Cyclone Shelter Information Database. Retrieved November 14, 2014, from http://www.dmic.org.bd/csdb/

Chalmers, A. F. (1999). What is this thing called science? (3rd ed.). Buckingham: Open University Press.

Chan, J. C. L. (1982). On the Physical Processes Responsible for Tropical Cyclone Motion (Atmospheric Science Paper No. 358). Colorado State University, Ft. Collins, Colorado, USA.

Chan, J. C. L., \& Kepert, J. D. (Eds.). (2010). Global Perspectives on Tropical Cyclones: From Science to Mitigation. World Scientific.

Chan, J. C. L., \& Lam, H. (1989). Performance of the ECMWF Model in Predicting the Movement of Typhoon Wayne (1986). Weather and Forecasting, 4(2), 234-245. http://doi.org/10.1175/1520-0434(1989)004<0234:POTEMI>2.0.CO;2

Chen, S. S., Zhao, W., Donelan, M. A., Price, J. F., \& Walsh, E. J. (2007). The CBLASTHurricane Program and the Next-Generation Fully Coupled Atmosphere-WaveOcean Models for Hurricane Research and Prediction. Bulletin of the American Meteorological Society, 88(3), 311-317. http://doi.org/10.1175/BAMS-88-3-311

Chowdhury, K. M. M. H. (2002). Cyclone preparedness and management in Bangladesh. In Improvement of early warning system and responses in Bangladesh towards total disaster risk management approach (pp. 115-119). Savar, Dhaka: BPATC.

Cione, J. J., \& Uhlhorn, E. W. (2003). Sea Surface Temperature Variability in Hurricanes: Implications with Respect to Intensity Change. Monthly Weather Review, 131(8), 1783-1796.

Cireşan, D., Meier, U., Masci, J., Gambardella, L. M., \& Schmidhuber, J. (2011). Flexible, high performance convolutional neural networks for image classification. In IJCAI Proceedings-International Joint Conference on Artificial Intelligence (Vol. 22, pp. 1237-1242). Retrieved from http://people.idsia.ch/ juergen/ijcai2011.pdf

Cireşan, D., Meier, U., Masci, J., \& Schmidhuber, J. (2011). A committee of neural networks for traffic sign classification. In The 2011 International Joint Conference on Neural Networks (IJCNN) (pp. 1918-1921). http://doi.org/10.1109/IJCNN.2011.6033458

Cireşan, D., Meier, U., Masci, J., \& Schmidhuber, J. (2012). Multi-column deep neural network for traffic sign classification. Neural Networks, 32, 333-338. http://doi.org/10.1016/j.neunet.2012.02.023

Cireşan, D., Meier, U., \& Schmidhuber, J. (2012). Multi-column deep neural networks for image classification. In 2012 IEEE Conference on Computer Vision and Pattern Recognition (CVPR) (pp. 3642-3649). http://doi.org/10.1109/CVPR.2012.6248110

Cooper, G. A., \& Falvey, R. J. (2010). Annual Tropical Cyclone Report 2009 (Technical) (p. 109). Hawaii. Retrieved from http://www.usno.navy.mil/NOOC/nmfc$\mathrm{ph} / \mathrm{RSS} / \mathrm{jtwc} / \mathrm{atcr} / 2009$ atcr/2009atcr.pdf

Coppola, D. P. (2006). Introduction to international disaster management. ButterworthHeinemann. 
CRED. (2013). EM-DAT: The OFDA/CRED International Disaster Database. Retrieved January 14, 2014, from http://www.emdat.be/database

Creswell, J. W. (2003). Research design: qualitative, quantitative and mixed methods approaches $(2 \mathrm{nd})$ edition. Thousand Oaks: Sage Publications.

Dam, M., \& Saraf, D. N. (2006). Design of neural networks using genetic algorithm for online property estimation of crude fractionator products. Computers \& Chemical Engineering, 30(4), 722-729. http://doi.org/10.1016/j.compchemeng.2005.12.001

Dash, N., \& Gladwin, H. (2007). Evacuation decision making and behavioral responses: Individual and household. Natural Hazards Review, 8, 69.

Davis, C., Wang, W., Chen, S. S., Chen, Y., Corbosiero, K., DeMaria, M., ... Michalakes, J. (2008). Prediction of landfalling hurricanes with the advanced hurricane WRF model. Mon. Wea. Rev., 136(6).

Davis, I., Sanderson, D., Parker, D., Stack, J., \& Lee, B. (1998). The dissemination of warnings. Thomas Telford.

DDM (Department of Disaster Management). (2009). New signaling system for cyclone and disaster message for public (original in bengali) (p. 38). Dhaka, Bangladesh: Ministry of Food and Disaster Management.

DDM (Department of Disaster Management). (2013). Emergency Preparedness Plan for Cyclone Bangladesh (p. 118). Dhaka, Bangladesh.

Debsarma, S. K. (1999). Cyclone forecasting and its constraints for the Bay of Bengal (p. 7). Presented at the Constraints of Weather Forecasting in Respective Country of the SAARC Region, SMRC, Dhaka.

Debsarma, S. K. (2001). Cyclone and Its Warning System in Bangladesh (p. 17). Presented at the National disaster reduction day, Dhaka, Bangladesh.

Deb, S. K., Kishtawal, C. M., \& Pal, P. K. (2009). Impact of Kalpana-1-Derived Water Vapor Winds on Indian Ocean Tropical Cyclone Forecasts. Monthly Weather Review, 138(3), 987-1003. http://doi.org/10.1175/2009MWR3041.1

DeMaria, M. (1996). The effect of vertical shear on tropical cyclone intensity change. $J$. Atmos. Sci, 53(14), 2076-2088.

DeMaria, M. (2009). A Simplified Dynamical System for Tropical Cyclone Intensity Prediction. Mon. Wea. Rev., 137(1), 68-82. http://doi.org/10.1175/2008MWR2513.1

Demaria, M., \& Kaplan, J. (1994). Sea Surface Temperature and the Maximum Intensity of Atlantic Tropical Cyclones. Journal of Climate, 7(9), 1324-1334. http://doi.org/10.1175/1520-0442(1994)007<1324:SSTATM>2.0.CO;2

DeMaria, M., Kaplan, J., \& Baik, J.-J. (1993). Upper-Level Eddy Angular Momentum Fluxes and Tropical Cyclone Intensity Change. Journal of the Atmospheric Sciences, 50(8), 1133-1147. http://doi.org/10.1175/15200469(1993)050<1133:ULEAMF $>2.0 . \mathrm{CO} ; 2$

DeMaria, M., Knaff, J. A., \& Sampson, C. (2007). Evaluation of long-term trends in tropical cyclone intensity forecasts. Meteorology and Atmospheric Physics, 97(1-4), 19-28. http://doi.org/10.1007/s00703-006-0241-4

DeMaria, M., Mainelli, M., Shay, L. K., Knaff, J. A., \& Kaplan, J. (2005). Further Improvements to the Statistical Hurricane Intensity Prediction Scheme (SHIPS). Weather \& Forecasting, 20(4), 531-543.

Despagne, F., \& Massart, D. L. (1998). Neural networks in multivariate calibration. The Analyst, 123(11), 157-178.

DMB (Disaster Management Bureau). (2010). National Plan for Disaster Management 2010-2015 (p. 103). Dhaka, Bangladesh. 
Douglas, M., \& Wildavsky, A. (1983). Risk and culture: an essay on the selection of technical and environmental dangers. University of California Press.

Drabek, T. E. (1999). Understanding disaster warning responses. The Social Science Journal, 36(3), 515-523. http://doi.org/10.1016/S0362-3319(99)00021-X

Drabek, T. E., \& Hoetmer, G. J. (1991). Emergency management: principles and practice for local government. International City Management Association.

Dunion, J. P., \& Velden, C. S. (2004). The Impact of the Saharan Air Layer on Atlantic Tropical Cyclone Activity. Bulletin of the American Meteorological Society, 85(3), 353-365. http://doi.org/10.1175/BAMS-85-3-353

Dvorak, V. F. (1972). A technique for analysis and forecasting of tropical cyclone intensities from satellite image (Technical No. NESS 36) (p. 15pp). NOAA.

Dvorak, V. F. (1975). Tropical Cyclone Intensity Analysis and Forecasting from Satellite Imagery. Mon. Wea. Rev., 103(5), 420-430.

Dvorak, V. F. (1984). Tropical cyclone intensity analysis using satellite data (No. NESDIS 11) (p. 45pp). NOAA.

Dvorak, V. F. (1995). Tropical clouds and cloud systems observed in satellite imagery: Tropical cyclones. Workbook Vol. 2, 359pp.

Edwards, Y. D., \& Allenby, G. M. (2003). Multivariate Analysis of Multiple Response Data. Journal of Marketing Research, 40(3), 321-334.

http://doi.org/10.1509/jmkr.40.3.321.19233

Emanuel, K., DesAutels, C., Holloway, C., \& Korty, R. (2004). Environmental Control of Tropical Cyclone Intensity. Journal of the Atmospheric Sciences, 61(7), 843-858. http://doi.org/10.1175/1520-0469(2004)061<0843:ECOTCI $>2.0 . C O ; 2$

Eriksson, H., Morin, M., Ekberg, J., Jenvald, J., \& Timpka, T. (2009). Assumptions Management in Simulation of Infectious Disease Outbreaks. AMIA Annual Symposium Proceedings, 2009, 173-177.

Essen, D. V., Anderson, C. H., \& Felleman, D. J. (1992). Information processing in the primate visual system: an integrated systems perspective. Science, 255(5043), 419423. http://doi.org/10.1126/science. 1734518

Evans, A. D., \& Falvey, R. J. (2012). 2012 Annual Tropical Cyclone Report (Annual report) (p. 117). Pearl Harbor, Hawaii.

Fasel, B. (2002). Robust face analysis using convolutional neural networks. In 16th International Conference on Pattern Recognition, 2002. Proceedings (Vol. 2, pp. 40-43 vol.2). http://doi.org/10.1109/ICPR.2002.1048231

Feibleman, J. K. (1966). Technology as skills. Technology and Culture, 7(3), 318-328.

FEMA (Federal Emergency Management Agency). (2010). Evacuation Plans. Retrieved November 30, 2010, from http://www.fema.gov/plan/prepare/evacuation.shtm

Feng, B., \& Liu, J. N. K. (2004). Semo-Mamo, A 3-phase module to compare tropical cyclone satellite images using a modified Hausdorff distance. In Proceedings of 2004 International Conference on (Vol. 6, pp. 3808-3813). Shanghai, China.

Fereday, J., \& Muir-Cochrane, E. (2008). Demonstrating Rigor Using Thematic Analysis: A Hybrid Approach of Inductive and Deductive Coding and Theme Development. International Journal of Qualitative Methods, 5(1), 80-92.

Flatau, M., Schubert, W. H., \& Stevens, D. E. (1994). The Role of Baroclinic Processes in Tropical Cyclone Motion: The influence of Vertical Tilt. Journal of the Atmospheric Sciences, 51(18), 2589-2601. http://doi.org/10.1175/15200469(1994)051<2589:TROBPI >2.0.CO;2

Franklin, J. L. (2009). 2008 National Hurricane Center Forecast Varification Report (Technical) (p. 71). Florida. 
Franklin, J. L. (2010). 2009 National Hurricane Center Forecast Varification Report (Technical) (p. 71). Florida.

Frank, N. L., \& Husain, S. A. (1971). The deadliest tropical cyclone in history. Bulletin of the American Meteorological Society, 52(6), 438-445.

Fujiwhara, S. (1923). On the growth and decay of vortical systems. Q. J. R. Meteorol. Soc, 49(206), 75-104. http://doi.org/10.1002/qj.49704920602

Fukushima, K. (1980). Neocognitron: A self-organizing neural network model for a mechanism of pattern recognition unaffected by shift in position. Biological Cybernetics, 36(4), 193-202. http://doi.org/10.1007/BF00344251

Fukushima, K. (1988). Neocognitron: A hierarchical neural network capable of visual pattern recognition. Neural Networks, 1(2), 119-130. http://doi.org/10.1016/08936080(88)90014-7

Fukushima, K. (2013). Artificial vision by multi-layered neural networks: Neocognitron and its advances. Neural Networks, 37, 103-119. http://doi.org/10.1016/j.neunet.2012.09.016

Fukushima, K., \& Miyake, S. (1982). Neocognitron: A new algorithm for pattern recognition tolerant of deformations and shifts in position. Pattern Recognition, 15(6), 455-469. http://doi.org/10.1016/0031-3203(82)90024-3

Garcia, C., \& Delakis, M. (2004). Convolutional face finder: a neural architecture for fast and robust face detection. IEEE Transactions on Pattern Analysis and Machine Intelligence, 26(11), 1408-1423. http://doi.org/10.1109/TPAMI.2004.97

Gentry, R. C., Rodgers, E., Steranka, J., \& Shenk, W. E. (1980). Predicting Tropical Cyclone Intensity Using Satellite-Measured Equivalent Blackbody Temperatures of Cloud Tops. Monthly Weather Review, 108(4), 445-455. http://doi.org/10.1175/1520-0493(1980)108<0445:PTCIUS >2.0.CO;2

Gladwin, H., Lazo, J. K., Morrow, B. H., Peacock, W. G., \& Willoughby, H. E. (2007). Social science research needs for the hurricane forecast and warning system. Natural Hazards Review, 8, 87.

GoB (Government of Bangladesh). (2008). Cyclone Sidr in Bangladesh: damage, loss and needs assessment for disaster recovery and reduction (p. 182). Dhaka, Bangladesh.

Goni, G., DeMaria, M., Knaff, J., Sampson, C., Ginis, I., Bringas, F., ... Halliwell, G. (2009). Applications of Satellite-Derived Ocean Measurements to Tropical Cyclone Intensity Forecasting.

Goni, G. J., \& Trinanes, J. A. (2003). Ocean thermal structure monitoring could aid in the intensity forecast of tropical cyclones. Eos, Transactions American Geophysical Union, 84(51), 573-578. http://doi.org/10.1029/2003EO510001

Gopalakrishnan, S., Liu, Q., Marchok, T., Sheinin, D., Surgi, N., Tong, M., ... Zhang, X. (2011). Hurricane Weather Research and Forecasting (HWRF) model: 2011 scientific documentation. L. Bernardet, Ed.

Gray, W. M. (1968). Global view of the origin of tropical disturbances and storms. Mon. Wea. Rev, 96(10), 669-700.

Gray, W. M., Neumann, C., \& Tsui, T. L. (1991). Assessment of the Role of Aircraft Reconnaissance on Tropical Cyclone Analysis and Forecasting. Bulletin of the American Meteorological Society, 72(12), 1867-1883. http://doi.org/10.1175/15200477(1991)072<1867:AOTROA>2.0.CO;2

Guha-Sapir, D., \& Lechat, M. F. (1986). Information systems and needs assessment in natural disasters: an approach for better disaster relief management. Disasters, $10(3), 232-237$. 
Guion, L. A., Diehl, D. C., \& McDonald, D. (2011). Conducting an in-depth interview. Retrieved from http://edis.ifas.ufl.edu/fy393

Haddow, G. D., Bullock, J. A., \& Coppola, D. P. (2008). Introduction to emergency management. Butterworth-Heinemann.

Halliwell, G. R., Shay, L. K., Jacob, S. D., Smedstad, O. M., \& Uhlhorn, E. W. (2008). Improving Ocean Model Initialization for Coupled Tropical Cyclone Forecast Models Using GODAE Nowcasts. Monthly Weather Review, 136(7), 2576-2591. http://doi.org/10.1175/2007MWR2154.1

Haque, C. E. (1995). Climatic hazards warning process in Bangladesh: experience of, and lessons from, the 1991 April cyclone. Environmental Management, 19(5), 719-734.

Haque, C. E. (1997). Atmospheric Hazards Preparedness in Bangladesh: A Study of Warning, Adjustments and Recovery from the April 1991 Cyclone. Natural Hazards, 16, 181-202.

Haque, C. E., \& Blair, D. (1992). Vulnerability to tropical cyclones: evidence from the april 1991 cyclone in coastal bangladesh. Disasters, 16(3), 217-229. http://doi.org/10.1111/j.1467-7717.1992.tb00400.x

Haque, U., Hashizume, M., Kolivras, K., Overgaard, H. J., Das, B., \& Yamamoto, T. (2012). Reduced death rates from cyclones in Bangladesh: what more needs to be done? Bulletin of the World Health Organization, 90(2), 150-156. http://doi.org/10.2471/BLT.11.088302

Holland, G. J. (1983). Tropical cyclone motion: Environmental interaction plus a beta effect. J. Atmos. Sci, 40(2), 328-342.

Holland, G. J., \& Lander, M. (1993). The Meandering Nature of Tropical Cyclone Tracks. Journal of the Atmospheric Sciences, 50(9), 1254-1266. http://doi.org/10.1175/1520-0469(1993)050<1254:TMNOTC $>2.0 . C O ; 2$

Holliday, C. R., \& Thompson, A. H. (1979). Climatological Characteristics of Rapidly Intensifying Typhoons. Mon. Wea. Rev., 107(8), 1022-1034. http://doi.org/10.1175/1520-0493(1979)107<1022:CCORIT>2.0.CO;2

Hong, Y., Hsu, K.-L., Sorooshian, S., \& Gao, X. (2004). Precipitation estimation from remotely sensed imagery using an artificial neural network cloud classification system. Journal of Applied Meteorology, 43(12).

Hope, J. R., \& Neumann, C. J. (1977). A survey of worldwide tropical cyclone prediction models (pp. 122-125). Presented at the Eleventh AMS Technical Conference on Hurricanes and Tropical Meteorology, Miami Beach, FL.

Hossain, M. Z., Islam, M. T., Sakai, T., \& Ishida, M. (2008). Impact of tropical cyclones on rural infrastructures in Bangladesh. Agricultural Engineering International: CIGR Journal, 1-13.

Ho, S. S., \& Talukder, A. (2008). Automated cyclone discovery and tracking using knowledge sharing in multiple heterogeneous satellite data (pp. 928-936).

Houze, R. A., Cetrone, J., Brodzik, S. R., Chen, S. S., Zhao, W., Lee, W.-C., ... Rogers, R. F. (2006). The Hurricane Rainband and Intensity Change Experiment: Observations and Modeling of Hurricanes Katrina, Ophelia, and Rita. Bulletin of the American Meteorological Society, 87(11), 1503-1521. http://doi.org/10.1175/BAMS-87-111503

Hubel, D. H., \& Wiesel, T. N. (1965). Receptive Fields and Functional Architecture in Two Nonstriate Visual Areas (18 and 19) of the Cat. Journal of Neurophysiology, 28(2), 229-289. 
Hubel, D. H., \& Wiesel, T. N. (1977). Functional Architecture of Macaque Monkey Visual Cortex. Proceedings of the Royal Society of London B: Biological Sciences, 198(1130), 1-59. http://doi.org/10.1098/rspb.1977.0085

Hubel, D. H., \& Wiesel, T. N. (1998). Early Exploration of the Visual Cortex. Neuron, 20(3), 401-412. http://doi.org/10.1016/S0896-6273(00)80984-8

Illich, I. (1974). Tools for Conviviality. Fontana, London: \{Marion Boyars Publishers\}. Retrieved from http://www.amazon.ca/exec/obidos/redirect?tag=citeulike0920\&path=ASIN/0714509744

Illich, I. (1978). The right to useful unemployment and its professional enemies. London: Boyars. Retrieved from http://library.wur.nl/WebQuery/clc/311839

Isaksen, L. (1997). Impact of ERS scatterometer data in the ECMWF 4D-Var assimilation system-preliminary studies. In Third ERS Symposium on Space at the service of our Environment (Vol. SP-414, pp. 1829-1851). Florence, Italy: ESA.

Isaksen, L., \& Janssen, P. A. E. M. (2004). Impact of ERS scatterometer winds in ECMWF's assimilation system. Quarterly Journal of the Royal Meteorological Society, 130(600), 1793-1814. http://doi.org/10.1256/qj.03.110

Isaksen, L., \& Stoffelen, A. (2000). ERS scatterometer wind data impact on ECMWF's tropical cyclone forecasts. IEEE T Geosci Remote, 38(4), 1885-1892.

Islam, M. S., Ullah, M. S., \& Paul, A. (2004). Community Response to Broadcast Media for Cyclone Warning and Disaster Mitigation: A Perception Study of Coastal People with Special Reference to Meghna Estuary in Bangladesh. Asian Journal of Water, Environment and Pollution, 1(1), 55-64.

Itti, L., \& Koch, C. (2001). Computational modelling of visual attention. Nature Reviews Neuroscience, 2(3), 194-203. http://doi.org/10.1038/35058500

Itti, L., Koch, C., \& Niebur, E. (1998). A Model of Saliency-Based Visual Attention for Rapid Scene Analysis. IEEE Transactions on Pattern Analysis and Machine Intelligence, 20(11), 1254-1259. http://doi.org/10.1109/34.730558

Jeffries, R. A., \& Miller, R. J. (1993). Tropical Cyclone Forecasters Reference Guide. 3. Tropical Cyclone Formation (No. AD-A265 216) (p. 45). Monterey, CA: NRL.

Jeffries, R. A., Sampson, C. R., Carr III, L. E., \& Chu, J. H. (1993). Tropical Cyclone Forecasters Reference Guide. 5. Numerical Track Forecast Guidance (No. ADA277 318). Monterey, CA: NRL.

Jin, L., Yao, C., \& Huang, X.-Y. (2008). A Nonlinear Artificial Intelligence Ensemble Prediction Model for Typhoon Intensity. Monthly Weather Review, 136(12), 45414554. http://doi.org/10.1175/2008MWR2269.1

Johnson, G. P., \& Lin, F. C. (1995). Hurricane tracking via backpropagation neural network. In Proceedings of IEEE International Conference on Neural Networks, 1995. (Vol. 2, pp. 1103-1106). 27 Nov.-1 Dec. Perth, Australia. http://doi.org/10.1109/ICNN.1995.487576

Johnson, R. B., \& Onwuegbuzie, A. J. (2004). Mixed Methods Research: A Research Paradigm Whose Time Has Come. Educational Researcher, 33(7), 14-26. http://doi.org/10.3102/0013189X033007014

Joseph, P. V. (1994). Tropical cyclone hazards and warning and disaster mitigation systems in India. Sadhana, 19(4), 551-566. http://doi.org/10.1007/BF02835639

JTWC. (2014). Tropical Cyclone Best Track Data Site. Retrieved March 31, 2015, from http://www.usno.navy.mil/NOOC/nmfc-ph/RSS/jtwc/best_tracks/

Kalnay, E., Kanamitsu, M., Kistler, R., Collins, W., Deaven, D., Gandin, L., ... Joseph, D. (1996). The NCEP/NCAR 40-Year Reanalysis Project. Bulletin of the American 
Meteorological Society, 77(3), 437-471. http://doi.org/10.1175/1520-

0477(1996)077<0437:TNYRP $>2.0 . \mathrm{CO} ; 2$

Kasiri, M. B., Aleboyeh, H., \& Aleboyeh, A. (2008). Modeling and Optimization of Heterogeneous Photo-Fenton Process with Response Surface Methodology and Artificial Neural Networks. Environ Sci Technol, 42(21), 7970-7975. http://doi.org/10.1021/es801372q

Keenan, T. D. (1982). A diagnostic study of tropical cyclone forecasting in Australia. Aust. Met. Mag, 30, 69-80.

Khan, K. S. (2005). A decade that was. Retrieved March 29, 2011, from http://www.gisdevelopment.net/application/natural_hazards/overview/nho0006.htm

Khan, M. A., Bhuyan, I. A., \& Rahman, M. M. (2010). Assessment of Cyclone Risk under the Changing Climatic Condition for the Coastal Areas of Bangladesh. In Dealing with Disasters Conference 2010 Linking Disasters and Development the next 10 years. Northumbria University, UK.

Kidder, S. Q., Goldberg, M. D., Zehr, R. M., DeMaria, M., Purdom, J. F. W., Velden, C. S., ... Kusselson, S. J. (2000). Satellite Analysis of Tropical Cyclones Using the Advanced Microwave Sounding Unit (AMSU). Bulletin of the American Meteorological Society, 81(6), 1241-1259. http://doi.org/10.1175/15200477(2000)081<1241:SAOTCU>2.3.CO;2

Kim, Y.-S., \& Yum, B.-J. B.-J. (2004). Robust design of multilayer feedforward neural networks: an experimental approach. Engineering Applications of Artificial Intelligence, 17(3), 249-263. http://doi.org/10.1016/j.engappai.2003.12.005

Kitano, H. (1994). Neurogenetic learning: an integrated method of designing and training neural networks using genetic algorithms. Physica D: Nonlinear Phenomena, 75(13), 225-238. http://doi.org/10.1016/0167-2789(94)90285-2

Knaff, J. A., \& Zehr, R. M. (2007). Reexamination of Tropical Cyclone Wind-Pressure Relationships. Weather and Forecasting, 22(1), 71-88. http://doi.org/10.1175/WAF965.1

Koch, C., \& Ullman, S. (1987). Shifts in Selective Visual Attention: Towards the Underlying Neural Circuitry. In L. M. Vaina (Ed.), Matters of Intelligence (pp. 115141). Springer Netherlands. Retrieved from http://link.springer.com/chapter/10.1007/978-94-009-3833-5_5

Kovordányi, R., \& Roy, C. (2009). Cyclone track forecasting based on satellite images using artificial neural networks. ISPRS Journal of Photogrammetry and Remote Sensing, 64(6), 513-521.

Krizhevsky, A., Sutskever, I., \& Hinton, G. E. (2012). ImageNet Classification with Deep Convolutional Neural Networks. In F. Pereira, C. J. C. Burges, L. Bottou, \& K. Q. Weinberger (Eds.), Advances in Neural Information Processing Systems 25 (pp. 1097-1105). Curran Associates, Inc.

Kuhn, T. S. (1970). The structure of scientific revolutions. University of Chicago press.

Kuo, H.-C., Williams, R. T., Chen, J.-H., \& Chen, Y.-L. (2001). Topographic Effects on Barotropic Vortex Motion: No Mean Flow. Journal of the Atmospheric Sciences, 58(10), 1310-1327. http://doi.org/10.1175/15200469(2001)058<1310:TEOBVM $>2.0$. CO;2

Kurihara, Y., Bender, M. A., Tuleya, R. E., \& Ross, R. J. (1995). Improvements in the GFDL hurricane prediction system. Mon. Wea. Rev, 123(9), 2791-2801.

Kurita, T., Nakamura, A., Kodama, M., \& Colombage, S. R. (2006). Tsunami public awareness and the disaster management system of Sri Lanka. Disaster Prevention and Management, 15(1), 92-110. 
Lajoie, F. A. (1976). On the direction of movement of tropical cyclones. Aust Meteorol Mag, 24(3).

Lajoie, F. A., \& Nicholls, N. (1974). A relationship between the direction and movement of tropical cyclones and the structure of their cloud systems (Technical No. Tech. Rep. No.11) (p. 22). Melbourne, Australia: Bureau of Meteorology.

Lakatos, I. (1978). The methodology of scientific research programmes (Vol. 1). Cambridge University Press. Retrieved from http://philpapers.org/rec/LAKTMO

Lander, M. A. (1995). The merger of two tropical cyclones. Monthly Weather Review, 123(7), 2260-2265.

Law, A. M., \& Kelton, W. D. (1991). Simulation Modeling and Analysis (2nd ed.). McGraw-Hill Education.

Lawrence, S., Giles, C. L., Tsoi, A. C., \& Back, A. D. (1997). Face recognition: a convolutional neural-network approach. IEEE Transactions on Neural Networks, 8(1), 98-113. http://doi.org/10.1109/72.554195

Lean, H. W., Clark, P. A., Dixon, M., Roberts, N. M., Fitch, A., Forbes, R., \& Halliwell, C. (2008). Characteristics of high-resolution versions of the Met Office Unified Model for forecasting convection over the United Kingdom. Monthly Weather Review, 136(9), 3408-3424.

LeCun, Y., \& Bengio, Y. (1995). Convolutional networks for images, speech, and time series. In The handbook of brain theory and neural networks (pp. 255-258). MA, USA: MIT Press.

LeCun, Y., Bengio, Y., \& Hinton, G. (2015). Deep learning. Nature, 521(7553), 436-444. http://doi.org/10.1038/nature14539

LeCun, Y., Boser, B., Denker, J. S., Henderson, D., Howard, R. E., Hubbard, W., \& Jackel, L. D. (1990). Handwritten Digit Recognition with a Back-Propagation Network. In Advances in Neural Information Processing Systems (pp. 396-404). Morgan Kaufmann.

LeCun, Y., Bottou, L., \& Bengio, Y. (1997). Reading checks with multilayer graph transformer networks. In , 1997 IEEE International Conference on Acoustics, Speech, and Signal Processing, 1997. ICASSP-97 (Vol. 1, pp. 151-154 vol.1). http://doi.org/10.1109/ICASSP.1997.599580

LeCun, Y., Bottou, L., Bengio, Y., \& Haffner, P. (1998). Gradient-based learning applied to document recognition. Proceedings of the IEEE, 86(11), 2278-2324. http://doi.org/10.1109/5.726791

LeCun, Y., Huang, F. J., \& Bottou, L. (2004). Learning methods for generic object recognition with invariance to pose and lighting. In Proceedings of the 2004 IEEE Computer Society Conference on Computer Vision and Pattern Recognition, 2004. CVPR 2004 (Vol. 2, pp. II-97-104 Vol.2). http://doi.org/10.1109/CVPR.2004.1315150

Lee, R. S. T., \& Liu, J. N. K. (2000). Tropical cyclone identification and tracking system using integrated neural oscillatory elastic graph matching and hybrid RBF network track mining techniques. IEEE T Neur Networ, 11(3), 680-689.

Legard, R., Keegan, J., \& Ward, K. (2003). In-depth interviews. Qualitative Research Practice: A Guide for Social Science Students and Researchers, 138-169.

Le Marshall, J. F., Leslie, L. M., Abbey Jr, R. F., \& Qi, L. (2002). Tropical cyclone track and intensity prediction: The generation and assimilation of high-density, satellitederived data. Meteorology and Atmospheric Physics, 80(1), 43-57.

Letson, D., Mileti, D. S., \& Lazo, J. K. (2007). Economic value of hurricane forecasts: An overview and research needs. Natural Hazards Review, 8, 78. 
Lin, F. C., Zhu, W., \& Sookhanaphibarn, K. (2012a). Detection and Early Warning of Tsunamis by Remote Sensing. Presented at the 5th International Tsunami Symposium, Ispra, Italy.

Lin, F. C., Zhu, W., \& Sookhanaphibarn, K. (2012b). Tsunami Detection by Remote Sensing in Infrared Space. Presented at the IGARSS 2012 Symposium, Munich, Germany.

Li, Z., \& Itti, L. (2011). Saliency and Gist Features for Target Detection in Satellite Images. IEEE Transactions on Image Processing, 20(7), 2017-2029. http://doi.org/10.1109/TIP.2010.2099128

Lo, S.-C. B., Chan, H.-P., Lin, J.-S., Li, H., Freedman, M. T., \& Mun, S. K. (1995). Artificial convolution neural network for medical image pattern recognition. Neural Networks, 8(7-8), 1201-1214. http://doi.org/10.1016/0893-6080(95)00061-5

Mallick, B., Witte, S. M., Sarkar, R., Mahboob, A. S., \& Vogt, J. (2009). Local Adaptation Strategies of a Coastal Community during Cyclone Sidr and their Vulnerability Analysis for Sustainable Disaster Mitigation Planning in Bangladesh. Journal of Bangladesh Institute of Planners, 2(0), 158-168. http://doi.org/10.3329/jbip.v2i0.9576

Martin, J. D., \& Gray, W. M. (1993). Tropical Cyclone Observation and Forecasting with and without Aircraft Reconnaissance. Weather and Forecasting, 8(4), 519-532. http://doi.org/10.1175/1520-0434(1993)008<0519:TCOAFW >2.0.CO;2

McBride, J. L., \& Holland, G. J. (1987). Tropical-Cyclone Forecasting: A Worldwide Summary of Techniques and Verification Statistics. Bulletin of the American Meteorological Society, 68, 1230-1238.

McClelland, J. L. (1993). The GRAIN Model: A Framework for Modeling the Dynamics of Information Processing. In Attention and Performance Xiv (pp. 655-688). The Mit Press.

McLuckie, B. F. (1970). The Warning System In Disaster Situations: A Selective Analysis. Retrieved March 29, 2011, from http://dspace.udel.edu:8080/dspace/handle/19716/1257

Merrill, R. T. (1987). An experiment in statistical prediction of tropical cyclone intensity change (NOAA Technical Memorandum No. NHC 34) (p. 33). NWS.

Miyan, M. A. (2006). Cyclone Disaster Mitigation in Bangladesh. Dhaka, Bangladesh.

Molteni, F., Buizza, R., Palmer, T. N., \& Petroliagis, T. (1996). The ECMWF ensemble prediction system: Methodology and validation. QJ Roy Meteor Soc, 122(529), 73119.

Mueller, K. J., DeMaria, M., Knaff, J., Kossin, J. P., \& Vonder Haar, T. H. (2006). Objective Estimation of Tropical Cyclone Wind Structure from Infrared Satellite Data. Weather and Forecasting, 21(6), 990-1005. http://doi.org/10.1175/WAF955.1

MWR (Ministry of Water Resources). (2005). Coastal Zone Policy (No. GoB) (p. 12). Dhaka, Bangladesh.

NCAR. (2014). Tropical Cyclone Guidance Project, Global Repository. Retrieved November 19, 2014, from http://www.ral.ucar.edu/hurricanes/repository/techlist/index.php

Neumann, C. J. (1972). An alternative to the HURRAN (hurricane analog) tropical cyclone forecast system (Technical No. NWS SR-62) (p. 24). Miami, Florida: NHC.

Neumann, C. J. (1979). A guide to Atlantic and eastern Pacific models for the prediction of tropical cyclone motion (NOAA Tech. Rep. No. NWS No. 932). Miami, Florida: NHC. 
Neumann, C. J. (1992). The joint typhoon warning center (JTWC92) model (No. SAIC Contract Rep. N 00014-90-C-6042 (Part 2)) (p. 85). Monterey, CA: SAIC.

Ngan, K. W., \& Chan, J. C. L. (1995). Tropical cyclone motion - steering vs. propagation (pp. 23-25). Presented at the 21st Tech. Conf. Hurricanes and Trop. Meteor., Miami, Florida, USA: Amer. Meteor. Soc.

NHC (National Hurricane Center). (2013). Saffir-Simpson Hurricane Wind Scale. Retrieved April 24, 2014, from http://www.nhc.noaa.gov/aboutsshws.php

NOAA, N. (2012). NHC Track and Intensity Models. Retrieved November 19, 2014, from http://www.nhc.noaa.gov/modelsummary.shtml

NOAA, N. (2015). NHC Data Archive. Retrieved March 15, 2015, from http://www.nhc.noaa.gov/data/\#hurdat

Nordin, I. (n.d.). Using Knowledge-on the rationality of science, technology and medicine.

Nowak, E., Jurie, F., \& Triggs, B. (2006). Sampling Strategies for Bag-of-Features Image Classification. In A. Leonardis, H. Bischof, \& A. Pinz (Eds.), Computer Vision ECCV 2006 (pp. 490-503). Springer Berlin Heidelberg.

Obasi, G. O. P. (1994). WMO's Role in the International Decade for Natural Disaster Reduction. Bulletin of the American Meteorological Society, 75(9), 1655-1661. http://doi.org/10.1175/1520-0477(1994)075<1655:WRITID>2.0.CO;2

O'Hara, S., \& Draper, B. A. (2011). Introduction to the Bag of Features Paradigm for Image Classification and Retrieval. arXiv:1101.3354 [cs]. Retrieved from http://arxiv.org/abs/1101.3354

Olander, T. L., \& Velden, C. S. (2007). The Advanced Dvorak Technique: Continued Development of an Objective Scheme to Estimate Tropical Cyclone Intensity Using Geostationary Infrared Satellite Imagery. Weather \& Forecasting, 22(2), 287-298. http://doi.org/10.1175/WAF975.1

O’Reilly, R. C. (1996). Biologically Plausible Error-Driven Learning Using Local Activation Differences: The Generalized Recirculation Algorithm. Neural Computation, 8(5), 895-938. http://doi.org/10.1162/neco.1996.8.5.895

O'Reilly, R. C. (1998). Six principles for biologically based computational models of cortical cognition. Trends Cogn Sci, 2(11), 455-462. http://doi.org/10.1016/S13646613(98)01241-8

O'Reilly, R. C., \& Munakata, Y. (2000). Computational explorations in cognitive neuroscience: understanding the mind by simulating the brain. MIT Press.

O'Reilly, R. C., Munakata, Y., Frank, M. J., Hazy, T. E., \& Contributors. (2012). Computational Cognitive Neuroscience (1st Edition).

O'Reilly, R. C., Wyatte, D., Herd, S., Mingus, B., \& Jilk, D. J. (2013). Recurrent Processing during Object Recognition. Frontiers in Psychology, 4. http://doi.org/10.3389/fpsyg.2013.00124

Parker, D. (1999). Criteria for evaluating the condition of a tropical cyclone warning system. Disasters, 23(3), 193-216.

Patton, M. Q. (1990). Qualitative evaluation and research methods. SAGE Publications, inc. Retrieved from http://psycnet.apa.org/psycinfo/1990-97369-000

Paul, A., \& Rahman, M. (2006). Cyclone Mitigation Perspectives in the Islands of Bangladesh: A Case of Sandwip and Hatia Islands. Coastal Management, 34(2), 199-215. http://doi.org/10.1080/08920750500531371

Paul, B. K. (2009). Why relatively fewer people died? The case of Bangladesh's Cyclone Sidr. Natural Hazards, 50(2), 289-304. 
Paul, B. K. (2012). Factors Affecting Evacuation Behavior: The Case of 2007 Cyclone Sidr, Bangladesh. The Professional Geographer, 64(3), 401-414. http://doi.org/10.1080/00330124.2011.609780

Paul, B. K., \& Dutt, S. (2010). Hazards warnings and response to evacuation orders: The case of Bangladesh's cyclone Sidr. Geographical Review, 100(3), 336-355. http://doi.org/10.1111/j.1931-0846.2010.00040.x

Paul, B. K., Rashid, H., Islam, M. S., \& Hunt, L. M. (2010). Cyclone evacuation in Bangladesh: Tropical cyclones Gorky (1991) vs. Sidr (2007). Environmental Hazards, 9(1), 89-101.

Persson, A. (2013). [Review of User guide to ECMWF forecast products, by E. Andersson]. ECMWF.

Phillips, B. D., \& Morrow, B. H. (2007). Social science research needs: Focus on vulnerable populations, forecasting, and warnings. Natural Hazards Review, 8, 61.

Pickle, J. (1991). Forecasting Short-Term Movement and Intensification of Tropical Cyclones Using Pattern-Recognition Techniques (No. Environmental Research Paper No. AD-A256705). MA, USA: Phillips Lab Hanscom AFB.

Pike, A. C. (1985). Geopotential Heights and Thicknesses as Predictors of Atlantic Tropical Cyclone Motion and Intensity. Monthly Weather Review, 113(6), 931-940. http://doi.org/10.1175/1520-0493(1985)113<0931:GHATAP >2.0.CO;2

Popper, K. R. (1959). The logic of scientific discovery. Oxford, England: Basic Books.

Powell, M. D., Vickery, P. J., \& Reinhold, T. A. (2003). Reduced drag coefficient for high wind speeds in tropical cyclones. Nature, 422(6929), 279-283. http://doi.org/10.1038/nature01481

Quarantelli, E. L. (1990). The Warning Process And Evacuation Behavior: The Research Evidence [Other]. Retrieved March 29, 2011, from http://dspace.udel.edu:8080/dspace/handle/19716/520

Rapp, F. (1974). Contributions to a Philosophy of Technology: Studies in the Structure of Thinking in the Technological Sciences. Reidel, Dordrecht.

Rawlins, F., Ballard, S. P., Bovis, K. J., Clayton, A. M., Li, D., Inverarity, G. W., ... Payne, T. J. (2007). The Met Office global four-dimensional variational data assimilation scheme. Quarterly Journal of the Royal Meteorological Society, 133(623), 347-362. http://doi.org/10.1002/qj.32

Reynolds, R. W., Rayner, N. A., Smith, T. M., Stokes, D. C., \& Wang, W. (2002). An Improved In Situ and Satellite SST Analysis for Climate. Journal of Climate, 15(13), 1609-1625. http://doi.org/10.1175/15200442(2002)015<1609:AIISAS > 2.0.CO;2

Rhome, J. R. (2007). Technical summary of the National Hurricane Center track and intensity models. Updated September, 12, 2007.

Riad, J. K., Norris, F. H., \& Ruback, R. B. (1999). Predicting Evacuation in Two Major Disasters: Risk Perception, Social Influence, and Access to Resources. Journal of Applied Social Psychology, 29(5), 918-934. http://doi.org/10.1111/j.15591816.1999.tb00132.x

Richardson, D. S. (2000). Skill and relative economic value of the ECMWF ensemble prediction system. QJ Roy Meteor Soc, 126(563), 649-667.

Rivolta, G., Marzano, F. S., Coppola, E., \& Verdecchia, M. (2006). Artificial neuralnetwork technique for precipitation nowcasting from satellite imagery. Advances in Geosciences, 7.

Rodgers, E. B., Mack, R., \& Hasler, A. F. (1983). A satellite stereoscopic technique to estimate tropical cyclone intensity. Monthly Weather Review, 111(8), 1599-1610. 
Rogers, R., Aberson, S., Black, M., Black, P., Cione, J., Dodge, P., ... Surgi, N. (2006). The Intensity Forecasting Experiment: A NOAA Multiyear Field Program for Improving Tropical Cyclone Intensity Forecasts. Bulletin of the American Meteorological Society, 87(11), 1523-1537. http://doi.org/10.1175/BAMS-87-111523

Roulstone, I., \& Norbury, J. (2013). Invisible in the Storm: The Role of Mathematics in Understanding Weather. Princeton University Press.

Roy, C., \& Kovordányi, R. (2012). Tropical cyclone track forecasting techniques - A review. Atmos Res, 104-105, 40-69. http://doi.org/10.1016/j.atmosres.2011.09.012

Roy, C., \& Kovordányi, R. (2015). Tropical Cyclone Track Forecasting. In Encyclopedia of Natural Hazards. Taylor \& Francis Group.

RSMC (Regional Specialized Meteorology Centers), New Delhi. (2013). Report on cyclonic disturbances over North Indian Ocean (p. 209). New Delhi.

Sahni, P., \& Ariyabandu, M. M. (Eds.). (2004). Disaster Risk Reduction in South Asia. PHI Learning Pvt. Ltd.

Sahni, P., \& Dhameja, A. (2004). Disaster Risk Reduction through Capacity Building of the Community and Panchayato Raj Institutions. In Disaster Risk Reduction in South Asia (pp. 162-184). PHI Learning Pvt. Ltd.

Saltzman, B. (1985). Satellite oceanic remote sensing. Academic Press.

Sampson, C. R., Jeffries, R. A., \& Neumann, C. J. (1995). Tropical Cyclone Forecasters Reference Guide 4. Tropical Cyclone Motion (p. 121). Monterey CA: NRL.

Retrieved from http://handle.dtic.mil/100.2/ADA302329

Sampson, C. R., Jeffries, R. A., Neumann, C. J., \& Chu, J.-H. (1995). Tropical Cyclone Forecasters Reference Guide 6. Tropical Cyclone Intensity, 48.

Santos, J. R. A. (2000). Getting the most out of multiple response questions. ACE, 2(1.4), $1-4$.

Schalkoff, R. J. (1997). Artificial neural networks. McGraw-Hill.

Sears, J., \& Velden, C. S. (2012). Validation of Satellite-Derived Atmospheric Motion Vectors and Analyses around Tropical Disturbances. Journal of Applied Meteorology and Climatology, 51(10), 1823-1834. http://doi.org/10.1175/JAMC-D12-024.1

Sermanet, P., \& LeCun, Y. (2011). Traffic sign recognition with multi-scale Convolutional Networks. In The 2011 International Joint Conference on Neural Networks (IJCNN) (pp. 2809-2813). http://doi.org/10.1109/IJCNN.2011.6033589

Shapiro, L. J. (1992). Hurricane Vortex Motion and Evolution in a Three-Layer Model. Journal of the Atmospheric Sciences, 49(2), 140-154. http://doi.org/10.1175/15200469(1992)049<0140:HVMAEI >2.0.CO;2

Shaw, R., Kobayashi, K. S. H., \& Kobayashi, M. (2004). Linking experience, education, perception and earthquake preparedness. Disaster Prevention and Management, 13(1), 39-49.

Shay, L. K., Goni, G. J., \& Black, P. G. (2000). Effects of a Warm Oceanic Feature on Hurricane Opal. Monthly Weather Review, 128(5), 1366.

Shoemaker, D. N. (1991). Characteristics of Tropical Cyclones Affecting the Philippine Islands (No. NOCC/JTWC Technical Note 91-1) (p. 35).

Shu, S., \& Wu, L. (2009). Analysis of the influence of Saharan air layer on tropical cyclone intensity using AIRS/Aqua data. Geophysical Research Letters, 36(9), L09809. http://doi.org/10.1029/2009GL037634

Simmons, A., Uppala, S., Dee, D., \& Kobayashi, S. (2007). ERA-Interim: New ECMWF reanalysis products from 1989 onwards. ECMWF Newsletter, 110(110), 25-35. 
Stark, J. D., Donlon, C. J., Martin, M. J., \& McCulloch, M. E. (2007). OSTIA : An operational, high resolution, real time, global sea surface temperature analysis system. In OCEANS 2007 - Europe (pp. 1-4). http://doi.org/10.1109/OCEANSE.2007.4302251

SWC (Storm warning Center). (2007). Tropical Cyclone Sidr Warning Message. Bangladesh Meteorological Department.

SWC (Storm warning Center). (2013). Tropical Cyclone Mahasen Warning Message. Bangladesh Meteorological Department.

Takeuchi, K. (2008). Hydro-meteorological disasters: Science and adaptation to climate change. Retrieved November 11, 2010, from http://www.cprm.gov.br/33IGC/1322577.html

Tallapragada, V., Bernardet, L., Biswas, M. K., Gopalakrishnan, S., Kwon, Y., Liu, Q., ... Zhang, X. (2014). Hurricane Weather Research and Forecasting (HWRF) Model: 2014 Scientific Document. DTC.

Tatham, P., Spens, K., \& Oloruntoba, R. (2009). Cyclones in Bangladesh-a case study of a whole country response to rapid onset disasters. In Proceedings of the 20th annual conference of the production and operations management society, Orlando, Florida, May (pp. 1-4).

Taylor, G. W., Fergus, R., LeCun, Y., \& Bregler, C. (2010). Convolutional Learning of Spatio-temporal Features. In K. Daniilidis, P. Maragos, \& N. Paragios (Eds.), Computer Vision - ECCV 2010 (pp. 140-153). Springer Berlin Heidelberg.

The World Bank. (2013). Emergency Cyclone Recovery and Restoration Project. Retrieved April 8, 2014, from http://www.worldbank.org/projects/P111272/emergency-2007cyclone-recovery-restoration-project?lang=en

Thomalla, F., \& Schmuck, H. (2004). "We All Knew that a Cyclone Was Coming": Disaster Preparedness and the Cyclone of 1999 in Orissa, India. Disasters, 28(4), 373-387. http://doi.org/10.1111/j.0361-3666.2004.00264.x

Van Horn, R. L. (1971). Validation of Simulation Results. Management Science, 17(5), 247-258. http://doi.org/10.1287/mnsc.17.5.247

Velden, C., Daniels, J., Stettner, D., Santek, D., Key, J., Dunion, J., ... Menzel, P. (2005). Recent Innovations in Deriving Tropospheric Winds from Meteorological Satellites. Bulletin of the American Meteorological Society, 86(2), 205-223. http://doi.org/10.1175/BAMS-86-2-205

Velden, C., Harper, B., Wells, F., Beven II, J. L., Zehr, R., Olander, T., ... McCrone, P. (2006). The Dvorak tropical cyclone intensity estimation technique: A satellitebased method that has endured for over 30 years. Bulletin of the American Meteorological Society, 87(9), 1195-1210.

Velden, C. S., Olander, T. L., \& Zehr, R. M. (1998). Development of an Objective Scheme to Estimate Tropical Cyclone Intensity from Digital Geo-stationary Satellite Inflated Imagery. Weather \& Forecasting, 13(1), 172.

Villmann, T., Merényi, E., \& Hammer, B. (2003). Neural maps in remote sensing image analysis. Neural Networks, 16(3-4), 389-403.

Wada, A., \& Usui, N. (2007). Importance of tropical cyclone heat potential for tropical cyclone intensity and intensification in the Western North Pacific. Journal of Oceanography, 63(3), 427-447. http://doi.org/10.1007/s10872-007-0039-0

Wang, B., \& Li, X.-F. (1992). The Beta Drift of Three-Dimensional Vortices: A Numerical Study. Monthly Weather Review, 120(4), 579-593. http://doi.org/10.1175/15200493(1992)120<0579:TBDOTD>2.0.CO;2 
Whittaker, H. (1979). Conprehensive Emergency Management: A Governor's Guide (p. 64). Washington, D. C.

WMO. (1989). Human Response to Tropical Cyclone Warnings and their Content (Tropical Cyclone Programme No. WMO Technical Document Report TCP-11). Geneva: World Meteorological Organisation.

Wolfe, J. M. (1994). Guided Search 2.0 A revised model of visual search. Psychonomic Bulletin \& Review, 1(2), 202-238. http://doi.org/10.3758/BF03200774

Wong, M. L. M., \& Chan, J. C. L. (2004). Tropical Cyclone Intensity in Vertical Wind Shear. Journal of the Atmospheric Sciences, 61(15), 1859-1876. http://doi.org/10.1175/1520-0469(2004)061<1859:TCIIVW>2.0.CO;2

Wright, P., \& McCarthy, J. (2009). Experience-Centered Design: Designers, Users, and Communities in Dialogue. Morgan \& Claypool Publishers.

Wu, A., Hsieh, W. W., \& Tang, B. (2006). Neural network forecasts of the tropical Pacific sea surface temperatures. Neural Networks, 19(2), 145-154. http://doi.org/10.1016/j.neunet.2006.01.004

Wu, C.-C., \& Emanuel, K. A. (1993). Interaction of a Baroclinic Vortex with Background Shear: Application to Hurricane Movement. Journal of the Atmospheric Sciences, 50(1), 62-76. http://doi.org/10.1175/15200469(1993)050<0062:IOABVW >2.0.CO;2

Wu, C.-C., Lee, C.-Y., \& Lin, I.-I. (2007). The Effect of the Ocean Eddy on Tropical Cyclone Intensity. Journal of the Atmospheric Sciences, 64(10), 3562-3578. http://doi.org/10.1175/JAS4051.1

Wyatte, D., Curran, T., \& O'Reilly, R. (2012). The limits of feedforward vision: recurrent processing promotes robust object recognition when objects are degraded. Journal of Cognitive Neuroscience, 24(11), 2248-2261.

Wyatte, D., Herd, S., Mingus, B., \& O'Reilly, R. C. (2012). The Role of Competitive Inhibition and Top-Down Feedback in Binding during Object Recognition. Frontiers in Psychology, 3, 182. http://doi.org/10.3389/fpsyg.2012.00182

Yang, Y., \& Wang, J. (2005). An integrated decision method for prediction of tropical cyclone movement by using genetic algorithm. Science in China Series D: Earth Sciences, 48(3), 429-440.

Zeng, Z., Chen, L., \& Wang, Y. (2008). An Observational Study of Environmental Dynamical Control of Tropical Cyclone Intensity in the Atlantic. Monthly Weather Review, 136(9), 3307-3322. http://doi.org/10.1175/2008MWR2388.1

Zhang, X., Li, T., Weng, F., Wu, C. C., \& Xu, L. (2007). Reanalysis of western Pacific typhoons in 2004 with multi-satellite observations. Meteorology and Atmospheric Physics, 97(1-4), 3-18. http://doi.org/10.1007/s00703-006-0240-5

Zhang, X., Xiao, Q., \& Fitzpatrick, P. J. (2007). The Impact of Multisatellite Data on the Initialization and Simulation of Hurricane Lili's (2002) Rapid Weakening Phase. Monthly Weather Review, 135(2), 526-548. http://doi.org/10.1175/MWR3287.1

Zhao, Q., \& Koch, C. (2013). Learning saliency-based visual attention: A review. Signal Processing, 93(6), 1401-1407. http://doi.org/10.1016/j.sigpro.2012.06.014

Zou, J., Lin, M., Xie, X., Lang, S., \& Cui, S. (2010). Automated typhoon identification from quikscat wind data (pp. 4158-4161).

Zschau, J., \& Küppers, A. N. (Eds.). (2003). Early warning systems for natural disaster reduction. Springer. 


\section{Chapter 9}

\section{Articles}

Article 1. Roy, C., Sarkar, S. K., Åberg, J., \& Kovordanyi, R. (2015). The current cyclone early warning system in Bangladesh: Providers' and receivers' views.

International Journal of Disaster Risk Reduction, 12, 285-299.

http://doi.org/10.1016/j.ijdrr.2015.02.004

Article 2. Roy, C., \& Kovordányi, R. (2012). Tropical cyclone track forecasting techniques - A review. Atmospheric Research, 104-105, 40-69.

http://doi.org/10.1016/j.atmosres.2011.09.012

Article 3. Roy, C. and Kovordanyi, R. (2015). Tropical cyclone track forecasting. Encyclopedia of Natural Hazards, Taylor \& Francis.

Article 4. Kovordányi, R., Roy, C., \& Saifullah, M. (2009). Local Feature ExtractionWhat Receptive Field Size Should Be Used? In Proceedings of International Conference on Image Processing, Computer Vision and Pattern Recognition. 1316 July, Las Vegas, NV.

Article 5. Saifullah, M., Kovordanyi, R., \& Roy, C. (2010). Bidirectional Hierarchical Neural Networks: Hebbian Learning Improves Generalization. In Proceedings of the Fifth International Conference on Computer Vision Theory and Applications (Vol. 1, pp. 105-111). 17-21 May, Angers, France.

Article 6. Roy, C. and Kovordanyi, R. (manuscript) A biologically based machine learning approach to tropical cyclone intensity forecasting. 


\section{Articles}

The articles associated with this thesis have been removed for copyright reasons. For more details about these see:

http://urn.kb.se/resolve?urn=urn:nbn:se:liu:diva-123198 


\section{Appendices}

Appendix A. Cyclone track forecasting using biologically based ANNs.

Appendix B. TC signaling system for the maritime ports.

Appendix C. TC signaling system for the river ports.

Appendix D. Questions used for the in-depth interview.

Appendix E. Questionnaire used for the survey.

Appendix F. Pre-recorded warning messages.

Appendix G. Generalized TC signaling system. 


\section{Appendix A}

\section{Cyclone track forecasting using biologically based ANNs}

The study described in Appendix A was conducted as part of a Master's thesis project at Linköping University. The use of biologically based ANNs for TC track and intensity forecasting in the present research was inspired by the success of this early study in TC movement direction prediction (see also section 2.8). This study has also been published as research article:

Kovordányi, R., \& Roy, C. (2009). Cyclone track forecasting based on satellite images using artificial neural networks. ISPRS Journal of Photogrammetry and Remote Sensing, 64(6), 513-521. http://doi.org/10.1016/j.isprsjprs.2009.03.002. 


\title{
Cyclone track forecasting based on satellite images using artificial neural networks
}

\author{
Rita Kovordányi and Chandan Roy \\ Dept. of Computer and Information Science \\ Linköping University, SE-581 83 Linköping, Sweden
}

\begin{abstract}
Many places around the world are exposed to tropical cyclones and associated storm surges. In spite of massive efforts, a great number of people die each year as a result of cyclone events. To mitigate this damage, improved forecasting techniques must be developed. The technique presented here uses artificial neural networks to interpret NOAA-AVHRR satellite images. A multi-layer neural network, resembling the human visual system, was trained to forecast the movement of cyclones based on satellite images. The trained network produced correct directional forecast for $98 \%$ of test images, thus showing a good generalization capability. The results indicate that multi-layer neural networks could be further developed into an effective tool for cyclone track forecasting using various types of remote sensing data. Future work includes extension of the present network to handle a wide range of cyclones and to take into account supplementary information, such as wind speeds, water temperature, humidity, and air pressure.
\end{abstract}

Keywords: Cyclones, Tracking, Neural, Networks, Hazards, Meteorology

\section{Introduction}

A tropical cyclone is an area of low pressure that develops over tropical or subtropical waters. These systems form over all tropical oceans with the exception of the South Atlantic and the eastern South Pacific, east of about $140^{\circ} \mathrm{W}$ longitude. There are seven tropical cyclone basins where tropical cyclones form on a regular basis:

1. Atlantic basin

2. Northeast Pacific basin

3. North Indian basin

4. Southwest Indian basin

5. Southeast Indian / Australian basin

6. Australian / Southwest Pacific basin, and

7. Northwest Pacific basin

In their most intense state, these storms are called hurricanes in the Atlantic, typhoons in the western North Pacific and cyclones in the Bay of Bengal. These low-pressure systems draw their energy from the very warm surface waters. As the warm, moist air spirals counterclockwise in toward the center, the wind speeds increase reaching their maximum in the region surrounding the almost calm center of the cyclone. 
As most cyclones form in tropical seas and population densities are the greatest in the tropical regions, cyclones constitute a major hazard for a large number of people around the world (Cerveny and Newman, 2000). Well known disasters include the Bangladesh cyclones of November 12, 1970 and May 24, 1985 (both crossed the coast at Chittagong), hurricane Camille that hit USA on August 17, 1969 and cyclone Tracy that swept over the Australian coast on December 25, 1974, and more recently hurricane Katrina. These cyclones have each caused a large number of fatalities and widespread property damage. Frequent, but less intensive cyclones (having less wind velocity and lower surge levels) continue to cause human casualties and considerable economic damage in tropical countries. Substantial resources have been devoted around the world for researching, forecasting, and promoting socioeconomic preparedness for cyclones and cyclone generated disasters. The present paper focuses on automating the forecasting of cyclone tracks and thereby providing a more reliable basis for early warning systems.

\subsection{Existing techniques for cyclone forecasting}

Tropical Cyclone (TC) forecasting involves the prediction of several interrelated features, including the track of the cyclone, its intensity, estimation of resulting storm surge and rainfall and, of course, which areas are threatened. Among these features, forecasting the future track and intensity of tropical cyclones are considered to be the most important for avoiding casualties and mitigating property damage. Some widely used cyclone track forecasting techniques are summarized in Table 1.

Table 1. A sample of techniques used in various meteorological offices to forecast cyclone tracks (adapted from McBride and Holland, 1987).

\begin{tabular}{|c|c|c|c|c|c|c|}
\hline \multirow[t]{2}{*}{ Office } & \multicolumn{6}{|c|}{ Techniques } \\
\hline & Subjective & Analogue & Steering & Statistical & Numerical & Empirical \\
\hline Japan & $\mathrm{X}$ & & & $\mathrm{X}$ & $\mathrm{X}$ & \\
\hline Hong Kong & $\mathrm{X}$ & & $\mathrm{X}$ & $\mathrm{X}$ & & \\
\hline Philippines & $\mathrm{X}$ & & & $\mathrm{X}$ & $\mathrm{X}$ & \\
\hline Miami & $\mathrm{X}$ & $\mathrm{X}$ & & $X$ & $X$ & \\
\hline India & $\mathrm{X}$ & $\mathrm{X}$ & & $X$ & & \\
\hline Brisbane & $X$ & $X$ & $X$ & $X$ & & \\
\hline Fiji & $\mathrm{X}$ & & & & & \\
\hline Reunion & $X$ & & & & & \\
\hline Mauritius & $X$ & & & & & \\
\hline Mozambique & $\mathrm{X}$ & & & & & \\
\hline Madagascar & $X$ & & & & & \\
\hline Darwin & $X$ & $\mathrm{X}$ & $\mathrm{X}$ & $\mathrm{X}$ & & \\
\hline Guam & $X$ & $X$ & $X$ & $X$ & $\mathrm{X}$ & $\mathrm{X}$ \\
\hline
\end{tabular}

Subjective assessment: This technique includes synoptic reasoning, evaluation of expected changes in the large-scale surrounding flow fields, and subjective evaluation of the cyclone's steering current.

Analogue forecasts: Designated features of the cyclone, such as its latitude, longitude, intensity, maturity, and past motion, are compared to those of previous cyclones in the same region, so that one or more analogues can be selected. The cyclone movement is then derived from the previous analogues.

Steering current: The cyclone's steering current is determined using analysis of winds at specified points and altitudes around the cyclone. The actual forecast can be based either on 
simple regression analysis or on analysis of the advection and propagation of winds, incorporating linear interactions between the cyclone vortex and the background absolute vorticity (Holland, 1984).

Statistical technique: All statistical forecasting techniques are based on regression analysis. Here, historical patterns of previous storms, such as cloud patterns, intensity development, and previous storm tracks are adapted and applied to the present storm.

Dynamical: These techniques are based on numerical integration of mathematical equations that approximate the physical behavior of the atmosphere. This technique provides different results when it is applied on a regional or global scale.

Empirical: A skilled meteorologist has often developed an ability to detect overall patterns in climatological conditions and can assess how these may affect cyclone development. Manual forecasts made by a skilled meteorologist may complement other forecasting techniques. The success of this technique critically depends on the forecaster's experience.

The National Hurricane Center (NHC) of the USA and the Australia Bureau of Meteorology (2007) identify the following additional techniques for cyclone movement forecasting:

Persistence: This technique is useful for short-term forecasts and assumes that the cyclone will maintain its recent track. It is often used in combination with other techniques.

Satellite-based techniques: In this technique, track and intensity forecasts are based on the cloud patterns associated with the cyclone. Generally, the outer bands of cumulonimbus clouds indicate the future direction, and the pattern surrounding the cyclone eye indicates the future intensity of the cyclone.

Hybrid: Meteorological centers round the world often employ a combination of techniques to obtain a more accurate track forecast. For example, two or more of the above techniques are blended as a weighted sum where the weights are based on past performance of each forecasting technique (Naval Research Laboratory, 1999).

\subsubsection{Automated forecasting}

Like other forecasts, tropical cyclone forecasts are not free from error. Errors in registering the initial position and motion of a tropical cyclone can impact the accuracy of subsequent forecasts. Errors can also arise from the lack of a full understanding of the mechanisms behind the formation and growth of tropical cyclones and from the limitations of the forecasting techniques themselves. Mean track-forecast error is typically smaller for lower latitude cyclones moving west than it is for higher latitude cyclones in westerly winds or for those that are re-curving. In general, mean track forecast errors tend to increase with the forecast period and can be as much as $30 \%$ of the cyclone movement within this period. This means that the forecasted track can deviate from the cyclone's actual track by as much as 20 degrees.

Due to the highly complex interaction between factors affecting cyclone development, meteorological offices around the world try to automate much of the work involved in cyclone forecasting. The Automated Tropical Cyclone Forecasting System (ATCF), developed by the Naval Research Laboratory (NRL) in Monterey, California, is an example of an automated forecasting system (Naval Research Laboratory, 1999). This computerbased application is intended to automate and optimize much of the tropical cyclone 
forecasting process. It provides a means for tracking and intensity forecasting, as well as constructing messages and disseminating warnings.

\subsubsection{Satellite-based techniques}

During the 1980s, forecasting of the track and intensity of tropical cyclones was mainly based on statistical (regression) methods using general meteorological data. Later, in the early 1990s, remote sensing techniques began to be successfully used for cyclone forecasting (Marshall et al., 2002; Wells, 1987). Development of new techniques, such as the generation of high resolution atmospheric motion vectors (AMVs) from satellite images, and four-dimensional variational assimilation (4D-VAR) have reduced the error in forecasting a cyclone's track and intensity (Marshall et al., 2002).

Images of different channels obtained from weather satellites have their specialized uses in tracking and forecasting the intensity of tropical cyclones. Satellite images within the thermal infrared (IR) band can be used to forecast and analyze the cyclone's intensity (Kossin, 2003). In addition, Lau and Crane (1997) and Kishtawal et al. (2005) measured the intensity of tropical cyclones based on satellite images and data from a thermal microwave imager using non-linear data fitting. Present techniques can be developed further. For example, data from advanced microwave sounder units have better horizontal resolution and vertical temperature sounding abilities that provide an improved basis for temperature estimation compared to conventional microwave sounding units and IR satellite images (Knaff et al., 2000).

Satellite-based techniques can be used for forecasting both a cyclone's intensity and track. TC development can be analyzed by studying the cloud patterns and determining how they change with time. Repeated observations of a TC provide information on the intensity and the rate of growth or decay of the storm. This method of intensity analysis is based on the degree of spiraling in the cloud bands. The Dvorak model is based on the observation that intense cyclones have more spiral cloud bands (Dvorak, 1975). The Dvorak model for forecasting intensity is successful in most cases. However, it is based on subjective judgments and is unreliable in the sense that various weather stations around the world can arrive at discrepant results for the same cyclone. Velden et al. (1998) uses a computer-based algorithm named the Objective Dvorak Technique (ODT) to address this problem.

\section{A new method for automated cyclone track forecasting}

One technique that has not been previously used for cyclone track forecasting is artificial neural networks (ANN). This in spite the fact that ANN-techniques have been used in other remote sensing application areas, such as road network detection (Barsi and Heipke, 2003a), cloud detection (Jang et al., 2006), cloud motion detection (Brad and Letia, 2002a, $2002 \mathrm{~b}$ ), and precipitation forecasting based on aerial photographs and satellite images (Hong et al., 2004; Rivolta et al., 2006). One conceivable reason why ANN-techniques have not been applied to cyclone forecasting is that it is difficult to achieve robust network performance in this complex domain.

Previous research suggests that cloud patterns surrounding the cyclone are good indicators of the direction of cyclone movement. There is a tendency for TCs to move towards the downstream end of convective cloud bands in the outer circulation strips around the cyclone (Lajoie, 1976). Changes in the orientation of these cloud bands indicate that a similar change in cyclone movement direction may occur in the next 12-24 hours. Further, TCs do not continue towards cumulonimbus free sectors in the outer circulation. 
Fett and Brand (1975) also noted that rotation of gross cloud features, such as an elliptical cloud mass or a major outer band; provide a very good indication of cyclone direction changes during the next 24 hours. The above results indicate that a cyclone's track is indicated by the shape and relative position of the surrounding cumulonimbus clouds. More importantly, these features are visible in satellite images (Fig. 1). Detecting and categorizing these features using a neural network would therefore provide valuable inputs for use in automated cyclone forecasting. In this article, we demonstrate that neural networks can learn to detect the surrounding clouds that are present in the satellite images and can recognize how these clouds are positioned relative to the cyclone center.

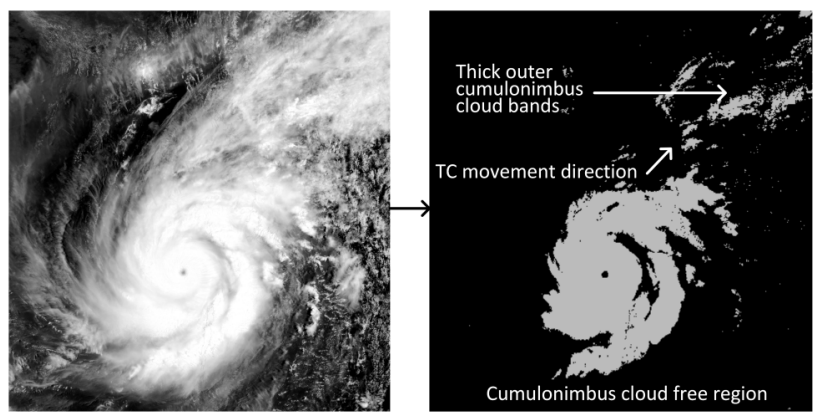

Fig 1. NOAA-AVHRR image of cyclone Mala showing a typical cloud pattern surrounding a cyclone. On the right side, cumulonimbus clouds extracted through reclassification. The dense cumulonimbus clouds, which are visible in the top right corner of the righthand-side image, indicate a strong upstream of hot, moist air. This upstream creates a low-pressure sector that fuels the cyclone and pulls it in a north-easterly direction.

\subsection{Robust pattern recognition inspired by human vision}

Previous examples using neural networks in remote sensing, such as detecting the presence of clouds and estimating air temperature based on satellite images deploy standard three layer feed forward networks (e.g. Barsi and Heipke, 2003b; Brad and Letia, 2002a, 2002b; Hong et al., 2004; Jang et al., 2006; Rivolta et al., 2006). However, the task for the neural network in the present study is to recognize an elongated shape in the image irrespective of random variations in its size and position, and it is difficult to achieve position and size invariance in standard three-layer networks.

\subsubsection{Biologically inspired image processing techniques}

Research in computer vision suggests that position and size invariance can be achieved by using deep multi-layer neural network architectures. These multi-layer architectures are to varying extents inspired by human vision. Somewhat simplified, the human visual system is made up of a series of transformational steps or processing levels. Each transformational step is reciprocally connected to both the predecessor and successor step (Felleman and Van Essen, 1991; Mishkin et al., 1983). This recurrent (i.e. bi-directional) communication, together with a cascade-like spreading of activation across the system (McClelland, 1993; O'Reilly, 1998), allows the various processing levels to work in concert and arrive at a consistent interpretation of visual input. 
Lower-level image processing in the human visual system is based on feature detectors. Individual feature detectors have limited receptive fields, that is, they are sensitive to a given feature within a limited part of the image. Interpretation of the complete visual image is achieved through parallel feature extraction across the image and stepwise hierarchical combination of these features into a coherent pattern. There are two sorts of computations going on during image processing:

1. Recognition of increasingly complex features, and

2. A stepwise increase of position, size, and rotation invariance.

As these two computations are difficult to achieve in the same computational step, biologically inspired networks for image processing often comprise pairs of layers containing simple cells and complex cells that implement computations 1 and 2, respectively (Fukushima and Miyake, 1982; Mutch and Lowe, 2008; Spratling, 2005). Simple-cell layers operate on a limited part of the image and combine features from the previous processing step into more complex shapes. In contrast, complex-cell layers achieve increased invariance by reacting uniformly to shapes that can be present anywhere within increasingly larger receptive fields.

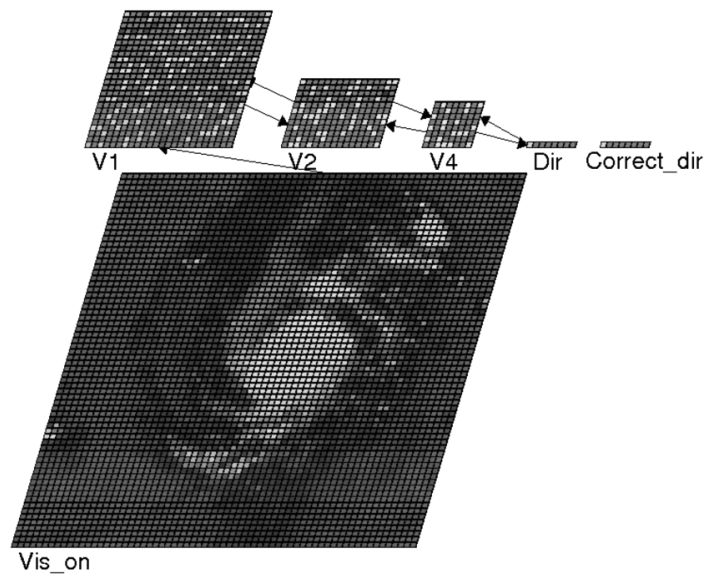

Fig 2. The five-layer network used in this study with bi-directional projections connecting subgroups of sending and receiving nodes. The network is constructed to detect constituent features in limited parts of the satellite image and integrate them into an overall shape. Note that the layer called 'Correct_dir' did not partake in computation or error calculation. It was used as an aid to display the correct answer for each image enabling us to visually compare the predicted versus the correct direction of cyclone movement. Calculation of training error took place in the Dir-layer, independently of the activation in Correct_dir.

As a rule, the first processing step in these artificial neural networks is tuned for the detection of line segments. These detectors are not developed during learning, but are instead pre-programmed, for example, using Gabor-filters (cf. e.g. Mutch and Lowe, 2008). In the present study, filled cloud segments turned out to be more appropriate as basic features. Detectors for these features were allowed to develop from the images through learning.

Existing mixed-cell multilayer networks have been demonstrated to achieve robust shape recognition, and these network architectures are among the most advanced techniques 
available. The question is, however, if shape and position invariance could be achieved using a simpler architecture involving only one type of cells, so that both feature detection and spatial integration are handled at each transformational step. When constructing our network, we used this simplified approach, with a down-scaled network architecture comprising five reciprocally connected layers (Fig. 2). We used one layer (and one type of cells) at each transformational step, instead of the commonly used interlacing of single-cell and complex-cell layers.

\subsubsection{Combined unsupervised and supervised learning}

Existing networks in the area of remote sensing have without exception been trained using the backpropagation of error learning algorithm (Barsi and Heipke, 2003b; Hong et al., 2004; Jang et al., 2006; Rivolta et al., 2006). As opposed to this, advanced networks in computer vision and image processing often use unsupervised learning for the training of low-level feature detectors.

Biologically based, as opposed to more loosely inspired networks combine unsupervised and supervised learning, as the human brain also seems to use a combination of learning methods (O’Reilly, 1998). To process perceptual information, humans use unsupervised model-based learning, which takes into account the statistical regularities that occur across various experiences. In addition, in order to learn how to map these regularities in the environment to an appropriate response, humans also use a form of error-driven task learning where the output (action) that is produced is compared to and slowly adapted over multiple experiences towards the desired output.

In this study, we employ a combination of model-based learning and error-driven task learning. Unsupervised, model-based learning is well suited for extracting cloud segments and more complex cloud patterns from the satellite images. Error-driven task learning is necessary for training the network to associate these patterns with a correct forecast of cyclone movement. One method of combining the two learning algorithms would be to let the first few layers in the network perform model-based learning, while subsequent layers would use error-driven task learning. However, if weights in the first few layers are changed independently of the task requirements, they could develop so as to work against the required input-output mapping, such as learning to detect features that are not relevant for the task at hand.

An efficient way to combine the two learning algorithms is to let both take place simultaneously at each layer, and to combine the outcome of the two algorithms for each learning cycle. There are both practical (faster learning) and theoretical advantages for combining the two learning algorithms in this way (cf. O’Reilly and Munakata, 2000).

\subsection{Biological transformational steps}

At the first transformational step in the human visual system (Fig. 3), the image captured by the retina is routed via the Lateral Geniculate Nucleus (LGN) to primary visual cortex (brain area V1). More exactly, the role of LGN is to bundle input from the two eyes and to mediate these signals to primary visual cortex V1. Brain area V1 contains specialized neurons that are sensitive to visual features, such as variously oriented line segments ${ }^{1}$. V1

1 Visual features constitute the basic building block of human vision, and reflect simple visual properties that are present in limited parts of the image. For example, recognition of the letter ' $\mathrm{A}$ ' 
neurons are dedicated to the processing of input from a limited part of the retinal image. This part of the retinal image corresponds to a specific region in external space, which comprises the neuron's receptive field (RF).

Feature detector neurons with the same RF cooperate in detecting various features located at the same part of space. For example, neurons that cover the same part of space may be sensitive to line segments with various orientation; these neurons are organized into one hypercolumn. Neighboring hypercolumns of feature detectors cover neighboring parts of space. Together, the matrix of hypercolumns can process the entire input image. The simultaneous activation of a specific combination of neurons within these hypercolumns reveals the presence of a combination of features in various parts of the image.

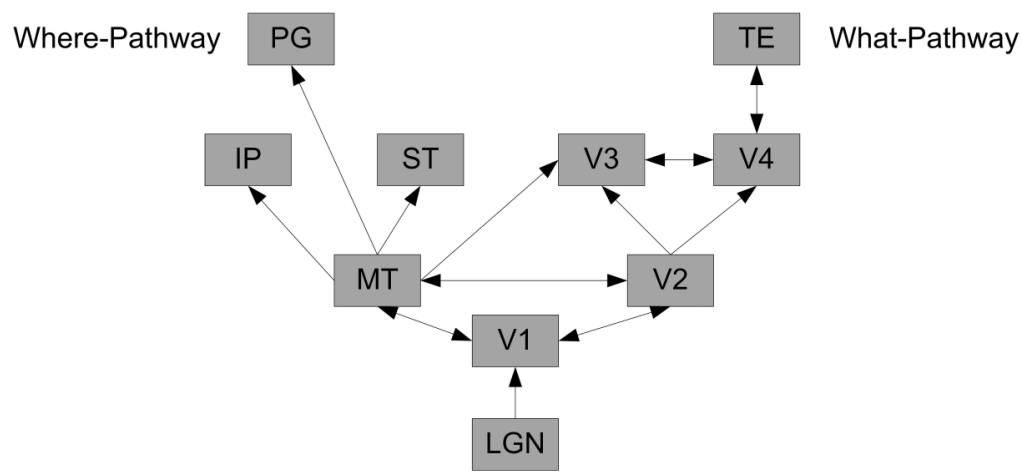

Fig 3. Architecture of the human visual system. The what- and where-pathways depicted in the figure are physically separated in the brain. The where-pathway runs in the dorsal (upper) part of the brain and is specialized for spatial processing of the size, location and orientation of objects. The what-pathway is located in the ventral (lower) part of the brain and implements color and shape detection (figure adapted from Felleman and Van Essen, 1991).

There is an inherent complexity in image processing based on feature detection. It is extremely difficult to reliably recognize a pattern of features when feature detection itself is underdetermined and has to be adjusted to variations in appearance (size, location, and orientation) of these features. There are simply too many degrees of freedom and these cannot be handled all at once. Instead, the brain implements a gradual transformation, cutting down the space of possible interpretations of the input image step by step. Hence, neighboring simple features represented in V1 are merged into slightly more complex (aggregate) features at the next step of processing in brain area V2. More specifically, detectors for these aggregate features react to the combined activation of simple feature detectors in V1. Also, V2 neurons indirectly receive input from a limited part of the retinal image, but these RFs now cover a slightly larger part of space.

builds on detection of the constituent line segments '/, '-', and ' '. These features are located in different parts of the input (the left, right, and middle part of the input). It is the combined presence of these features and their relative location that signals the presence of the letter 'A'. 
Through further step by step transformations (in areas V3, V4 and TE), features are gradually aggregated into more and more complex features. For each transformational step, the aggregated features will occupy larger and larger parts of the image. The advantage of this hierarchical approach is twofold: At each transformational step, feature detection needs only handle variations in location within a limited part of space (i.e. within the RF of the feature detector). Also, processing of the image is simplified as variations in size and to some extent orientation apply to simpler constituent features as opposed to complex overall patterns. Hence, step-by-step processing in a feature detector hierarchy makes the problem of the many degrees of freedom inherent to image processing more tractable.

\subsection{Architecture of our artificial neural network}

Robust pattern recognition can thus be achieved through stepwise processing in a bidirectionally connected multi-layer network. At the first processing level, shape recognition is based on detection and integration of characteristic constituent features, such as small cloud segments. The constituent features are smaller in size than the aggregate shape (the whole cloud pattern), and therefore can be detected by looking at limited parts of the image. The stepwise integration of features that we have implemented in our artificial neural network resembles the part of the human visual system that is responsible for pattern detection and shape recognition, which is commonly called the what-pathway (Creem and Proffitt, 2001; Goodale and Milner, 1992; Kosslyn, 1994) comprising brain areas V1 to IT (cf. Fig. 3).

By processing the image through a matrix of feature detector units, constituent features in different parts of the image can be extracted in parallel (O'Reilly and Munakata, 2000). For each feature that is detected, the network needs to abstract away from minor variations in position and size, but only within a small part of the image. Due to the task requirements, rotational variations are encoded as meaningful features at each stage of processing.

At subsequent stages of processing, the simpler constituent features are integrated into larger patterns forming more complex features. Recognition of these secondary features is based on the combined activation of feature detectors at the previous stage, and is not strictly dependent on which part of the image these activations originate from. For each additional transformational step, more position and size independency is achieved and more complex patterns can be recognized. At the final stages of processing, the constituent features can be integrated into an overall shape that is perceived to be rotated in a specific way. This information is then mapped onto a directional indicator showing the predicted movement of the cyclone.

\subsubsection{Enhanced generalization ability}

Through consecutive transformational steps, detection of shape and orientation becomes increasingly independent of the exact position and size of the features, and is instead based on how the constituent features are located and oriented relative to each other. Image processing is based on simple features, and these features are present also in novel satellite images. Satellite images not previously presented to the network would still elicit a correct response based on the detection of previously experienced and well-learned constituent features. Hence, step-by-step image processing using feature detectors should enhance the network's generalization capability. 


\section{Implementation of the network}

The network presented in this paper was implemented and trained using the artificial neural network simulation tool Leabra++, which has been specifically developed for modeling biologically based (brain-like) artificial neural networks (O'Reilly and Munakata, 2000). The network consisted of an input layer, three hidden layers, and an output layer (Fig. 2). All layers except the input layer were bi-directionally connected meaning that if a layer was sending input to another layer, it also received feedback from that layer. These feedback connections provided parallel constraint satisfaction capabilities, and were also used for error-driven learning. Connections were symmetrical so that if unit $x$ was sending input to unit $y$, then $\mathrm{x}$ also received feedback from $y$. This symmetry was preserved during training.

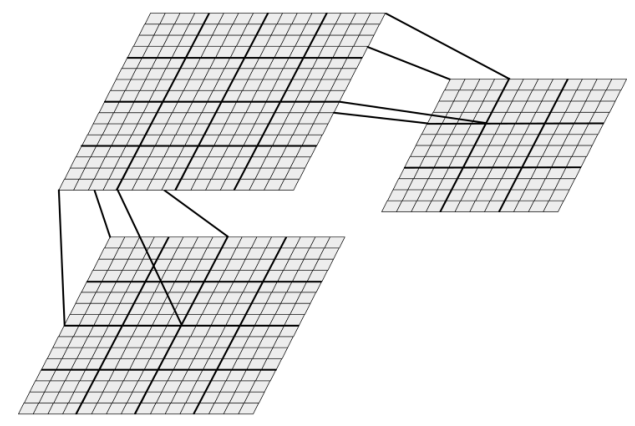

Fig 4. Selective communication between corresponding groups of nodes across layers. This communication structure resembles the limited receptive fields in the human visual system. For each transformational step, increasingly complex features can be extracted and these features originate from increasingly larger parts of the input.

The first receiving layer V1 was designed to encode simple visual features in the input image, such as small cloud segments. Nodes in this layer were grouped and connected to the input in such a way that each group would receive input only from a limited part of the image. Layer V1 thus consisted of $3 \times 3$ groups containing $8 \times 8$ units each. Each of these groups received input from a $26 \times 26$-pixel patch of the input image. The grouping of nodes and selective communication between groups of nodes across layers simulated the concept of limited receptive fields (RF) that can be found in the human visual system (Fig. 4). Note however, that 3.26 is greater than 66 pixels, which means that neighboring receptive fields slightly overlapped in order to maintain continuity of processing across various parts of the image. Using this network architecture, local information from different parts of the image could be processed in parallel. For each transformational step in the network, information was integrated taking into account larger and larger parts of the input image. Hence, layer V2 consisted of four neighboring groups of $8 \times 8$ units, where each group received input from four groups in layer V1, which in turn covered $46 \times 46$ pixels in the input image.

Finally, layer V4 comprised of $8 \times 8=64$ units, where each unit received input from all units in layer V2. At the final transformational step (V4 $\rightarrow$ Dir), the shape and orientation of the overall cloud pattern was transformed into a directional prediction indicating which direction the cyclone was most likely to move. Output from the network was represented by an $8 \times 1$ vector, where each bit (i.e. activation of one individual node) signified a direction 
ranging from $0^{\circ}$ to $315^{\circ}$ in $45^{\circ}$ increments. The low resolution of output reflected the relatively low resolution of the input images ( $66 \times 66$ pixels).

\subsection{Training data}

Training data were prepared by downsizing the original satellite images to $66 \times 66$ pixels, converting the resulting images into grayscale, and linearly transforming this grayscale image from a format where each pixel contained a value between 0 and 255 into a representation where pixel values were limited to the interval $[0,1]$. This transformation would thus map an original pixel value of 255 to a new pixel value of 1 . Similarly, an original pixel value of 127 would be transformed into a new pixel value of $127 / 255$, and so on. Note that this transformation preserved the information contained in the original image.

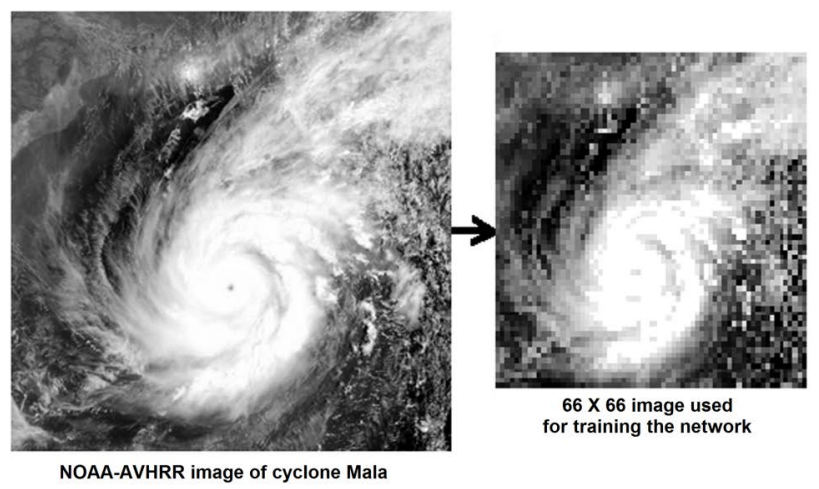

Fig 5. Example of NOAA-AVHRR image and, on the righthand-side, corresponding image downsized to 66 x 66 pixels. The downsized image was artificially rotated clockwise in $45^{\circ}$ increments. Each of the rotated images was zoomed and shifted in position in order to create variation in the cyclone's orientation, size, and location within the image.

The resulting image was then artificially rotated clockwise in $45^{\circ}$ increments, which resulted in eight rotated images. Each of the rotated images was zoomed in three ways (using zoom factors $0.8,1$, and 1.2), in order to create variation in the cyclone's size relative to the image frame. Finally, each of the rotated and zoomed images was shifted in position either zero, one, or two steps. The size of the steps was varied across training sessions so that we could compare network performance for the one-pixel step size with the three-pixel step size.

Position shifts were generated in eight directions (north, south, east, west, northeast, etc.). The resulting position of the cyclone could differ by as much as 12 pixels (when using 3-pixel steps for shifting), which amounted to almost one-fifth of the total image size. As a result, important information about the cyclone's surroundings would not be included from the edge of some images. To avoid moving the most informative part of the cyclone's surroundings outside the image frame, two-step position shifts in the same direction the cyclone was moving were automatically discarded. These transformations resulted in 1200 images.

From the total set of images, a random subset comprising approximately $5 \%$ of the images was set aside for later testing of network performance. The remaining $95 \%$ of the 
images were used for training the network. The correct direction in which a cyclone moved was determined by visual estimation using the original satellite image and was automatically calculated for the transformed images.

Visual estimation of the cyclone's movement was based on the general rule that it is most likely to move in a direction where there are cumulonimbus clouds in the outer circulation bands, as these signify a local area of low pressure (cf. Fig. 1). The network's task was to detect the same pattern as the human eye, and to use this information to predict the cyclone's future movement. In this way, the artificial neural network was required to play a role similar to a human expert at a meteorological department.

We were forced to use relatively low-resolution images (Fig. 5) due to computational resource limitations. We used commercially available $2.2 \mathrm{GHz}$ PCs for our network simulations. We reasoned that if the network would learn to predict cyclone movement based on these low-resolution images, this would be an excellent proof of the concept demonstrating the usefulness and robustness of ANN-techniques for cyclone track prediction.

\subsection{Information processing in the network}

For each input-output pair in the training set, the network went through two activation phases. During the minus phase, the network was presented with the input and then was allowed to settle into an activation state consistent with the input and with the weights of the network. During settling, weakly activated nodes were suppressed allowing at most $k$ nodes to be active within a group of nodes in the same group of units and/or the same layer. This mechanism is commonly referred to as $k$-winners-take-all (kWTA).

In the second plus phase, activation in the network was reset to zero and the network was presented with both the input and the correct output. During this second phase, the network settled into an activation state that reflected the correct ("should-have-been") activation of each node. By comparing the activations between the minus and the plus phases, an error was computed for each node.

The receiving weights for each node were adjusted in a direction that decreased this error. In addition, a weight change was also computed based on the co-activation of pairs of nodes. Pairs of sending and receiving nodes activated together during the plus phase were assigned a small positive weight change to enforce this tendency for co-activation. Likewise, a negative weight change was assigned to connections going from an inactive to an active node, and vice versa, in order to decrease the dissonance between these nodes. The final weight update for each connection reflected the sum of the error-driven and correlation-based weight change (see Equation 4).

Training of the network can be described in mathematical terms as follows. Weights in the network were limited to the interval $[0,1]$. Also, the activation of nodes was limited to the interval $[0,1]$. The activation function describing how strongly a node should react to input was given by the following biologically based, sigmoid-like (S-shaped) function (O'Reilly and Munakata, 2000):

$$
\begin{gathered}
y_{j}=\frac{\gamma\left[V_{m}-\Theta\right]_{+}}{\gamma\left[V_{m}-\Theta\right]_{+}+1}, \quad[z]_{+}=\left\{\begin{array}{l}
z \text { if } z \geq 0 \\
0 \text { if } z<0
\end{array}\right. \\
y_{j}=\text { activation of receiving node } j \\
\gamma=\text { gain }
\end{gathered}
$$

\section{Equation 1}




$$
\begin{aligned}
& V_{m}=\text { membrane potential } \\
& \Theta=\text { firing threshold }
\end{aligned}
$$

Learning in the network was based on a combination of Conditional Principal Component Analysis (CPCA), which is a form of unsupervised model-based learning algorithm (Hebbian learning) and Contrastive Hebbian learning (CHL), which is a biologically based alternative to backpropagation of error (O'Reilly, 1998; O'Reilly and Munakata, 2000):

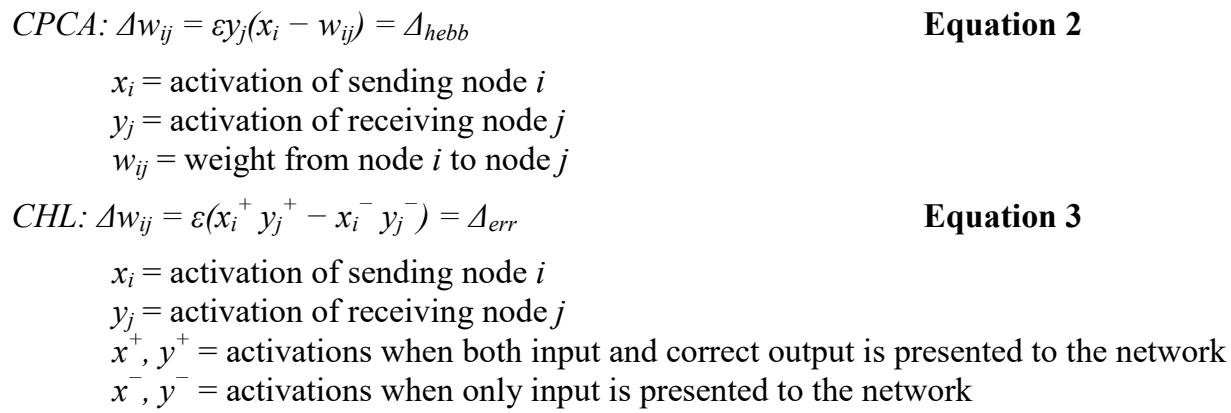

Equation 4

$\varepsilon=$ learning rate (initially set to 0.01 , and then gradually decreased during training)

$c_{\text {hebb }}=$ proportion of Hebbian learning (set to 0.01 in the present study)

\section{Results}

Training was run in epochs. Each epoch consisted of one round of the presentation of the complete training set, which comprised approximately $95 \%$ of the 1200 images. For each epoch, the images were permuted to avoid sequence learning effects. Error in network performance was defined as the difference between the correct output and the output produced by the network. The difference was calculated for each output unit, and then squared and summed into an overall error. The output layer in the network consisted of eight nodes, where each node represented a possible movement ranging from $0^{\circ}$ (north) to $315^{\circ}$ (northwest) in $45^{\circ}$ increments.

Performance error was measured in each of these eight nodes, as the network was required to activate one of these nodes, but not the others. The individual errors were squared (to eliminate differences in sign) and summed for the eight units producing a summed squared error (SSE) for each output produced by the network. Performance error for each epoch was measured as the SSE summed over all input-output pairs in the training set. We conducted multiple trainings to make sure that network training was stable, that is, independent of the initial random weights. In general, for all training runs, the training error (SSE) decreased rapidly and stabilized at around 20, after 10-15 epochs (Fig. 6). This implied an average error of 0.02 , divided among eight nodes, for each output produced by the network.

These results were obtained for images where the position of the cyclone center was varied by a maximum of $6 \%$ of the total image size. This means that when using the network for cyclone track prediction, the cyclone needs not be relatively precisely positioned in the canter of the image in order for the network to produce valid track predictions. To get a feeling for how well the network handled positional variations, we 
experimented with training the network using larger variations in the position of cyclone center. A typical training curve for such a training set is depicted with dashed line in Fig. 6.

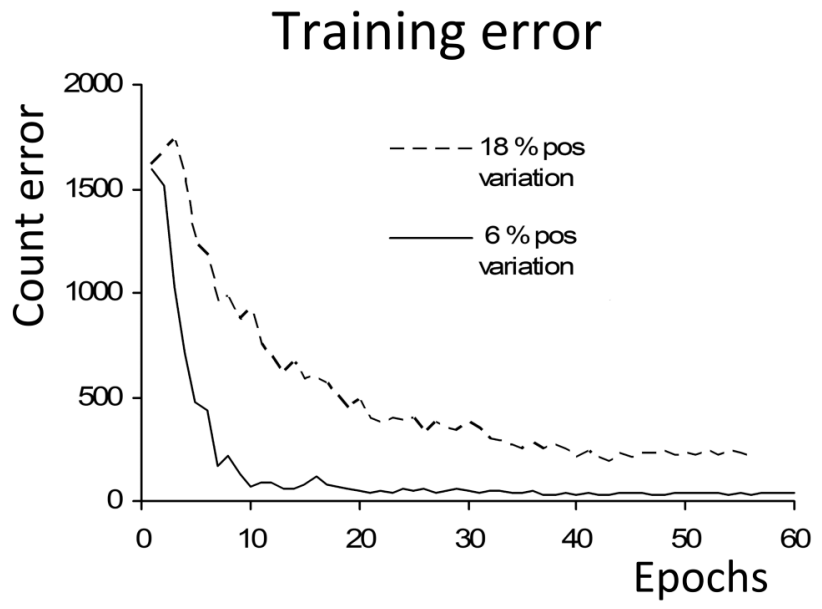

Fig 6. Sample training curves. Typically, the network reached a training error of 30 40 after 10-15 epochs of training when cyclone position was varied by $6 \%$ of the image size. The dashed line shows a sample training curve for input images where the cyclone canter varied in position by up to $18 \%$ of the image size.

\subsection{Network performance for novel images}

About $5 \%$ of the images were excluded from the training set and were presented to the network for the first time during testing. During testing, we recorded which images were and were not interpreted correctly. We obtained correct direction prediction for about $99 \%$ of the test images when the cyclone's center varied by $6 \%$ of the image size (Fig. 7a). When the position of the cyclone center varied more within the image frame $(18 \%$ of the image size), correct track predictions dropped to $84 \%$ of the test images (Fig. $7 \mathrm{~b}$ ).
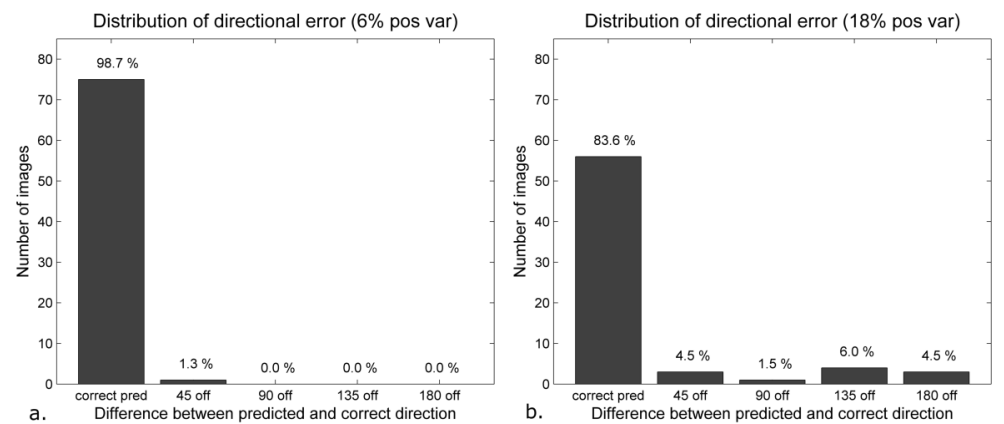

Fig 7. Sample distribution of errors for the test images. a. Directional errors when cyclone center varied by $6 \%$ of the total image size. The leftmost bar indicates correct cyclone track predictions for test images $(98.7 \%)$. b. Distribution of errors when cyclone position varied by $18 \%$. A stable pattern across multiple runs was the relatively few orthogonally incorrect predictions $\left(90^{\circ}\right.$ error $)$. 
For the image set that contained images with up to $18 \%$ positional variation of the cyclone center relative to the image frame, several training and testing sessions were conducted using different starting weights. We detected a reoccurring pattern: In about $5 \%$ of the cases, that is, for 3-4 of the 70-75 test images the network mistakenly predicted that the cyclone was moving opposite to the correct direction. In other words, the predicted direction erred by $180^{\circ}$ (Fig. 7 b). For a few images, the prediction erred by $45^{\circ}$. Finally, for 1-2 images, predicted direction was perpendicular to the correct direction. Hence, mistaken predictions tended to be either close to the correct direction $\left(45^{\circ} \mathrm{off}\right)$, or in the opposite direction (or directions close to this). Incorrect predictions that were oriented perpendicular to the correct direction were comparatively rare.

\section{Analysis of network performance}

The pattern of errors produced by the network (Fig. 7a and 7b) indicated that predictions were based on shape information, namely the elongated shape formed by the cyclone together with the cumulonimbus clouds that form on one side of the cyclone. The images fed into the network were relatively low-resolution ( $66 \times 66$ pixels), and it seems that for some of these images the network mistakenly recognized the elongated shape as being oppositely oriented (e.g. upside-down or mirrored left-to-right) resulting in predictions erred by approximately $180^{\circ}$. In contrast, the network made relatively few errors where predictions differed $90^{\circ}$ from the true direction of movement.

The internal workings of artificial neural networks are often considered to be impenetrable for the human analyst. An interesting question is therefore how the network performs cloud pattern recognition. For this, we need to understand which low-level features are extracted by the network when a satellite image is presented. What sort of lowlevel features the network has learned to detect is in turn reflected by the weight structure that has developed during training between the first input layer (Vis_on) and the next receiving layer (V1).
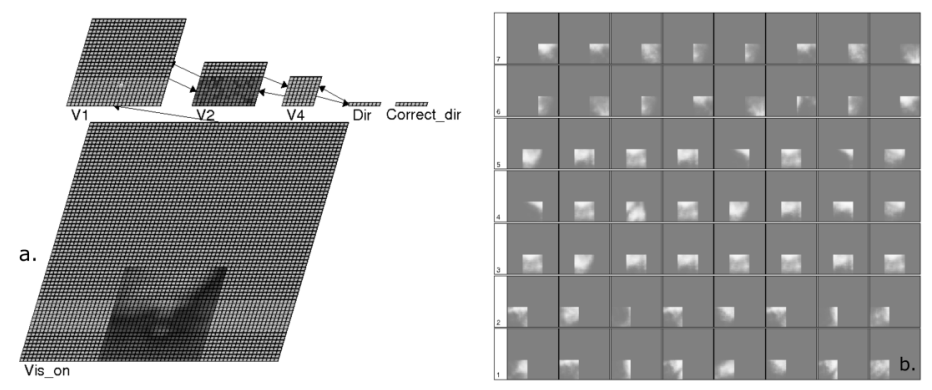

Fig 8. a. Receiving weights for a particular node in V1 developed during training. This node receives input from part of Vis_on (comprising the RF of this node) and also receives feedback signals from V2. Note that the weight pattern from Vis_on resembles a cloud segment and is the feature that this particular receiving node specializes for. $\mathbf{b}$.

Receiving weights for a group of neighboring nodes in V1. Block (x, y) shows the weight pattern from part of Vis_on into node $(\mathrm{x}, \mathrm{y})$ within a receiving group in V1.

The receiving (incoming) weights connecting to the individual receiving units in V1 can be plotted to see which pixels in the input image that this particular receiving unit has learned to react to. An example is shown in Fig. 8a, where the receiving weights have been 
visualized for one particular unit in V1. As can be seen in the figure, these weights indicate that the receiving unit has been specialized for detecting a particularly shaped cloud segment.

One way of visualizing the receiving weights for several units is to stack the weight matrices for neighboring receiving units within a receiving group into a matrix-like pattern. Hence, for example, the weight matrix for unit $(0,0)$ in V1, the leftmost bottom unit, would be placed in position $(0,0)$ in the stacked weight matrices plot. Looking at the stacked weight matrices plot (Fig. 8b), the division of labor in V1 becomes evident as various receiving units in V1 have been specialized for detecting different types of cloud segments. Depending on which of these features are present in the satellite image, the corresponding units in V1 will be activated, indicating the presence of a particular combination of features. Based on this activation pattern, subsequent layers (e.g. V2) will be able to recognize the overall cloud shape.

\section{Discussion}

The network was able to learn the task quickly (in 15-20 epochs) and this learning behavior was stable, which indicates that the network architecture (size and structure of layers and connection structure) was basically sound. Because we had limited computational resources available, we were forced to use relatively low-resolution input images (cf. Fig. 5). In spite of the low-resolution images ( $66 \times 66$ pixels) that were used, it was possible to train the network and achieve correct cyclone track predictions for $99 \%$ of the test images when small variations in the cyclone position were allowed. According to our analysis, the network was able to learn to interpret the shape of cloud patterns in the cyclone, and map the orientation of this overall shape to a correct movement direction. Most importantly, the network could achieve this irrespective of variations in the position and apparent size of the cyclone.

When larger variations in cyclone position were allowed, for about $10 \%$ of the test images, the network mistakenly interpreted the image as being upside-down or mirrored making predictions that erred by approximately $180^{\circ}$. This type of error implies that the network successfully recognized the elongated shape that was formed by the cyclone and the surrounding clouds, but that the orientation of this overall shape was mistakenly recognized as being upside-down and/or left-right mirrored. These mistakes are most likely caused by the low-resolution images that were used as input.

In a relatively few cases $(1.5 \%$ when cyclone position was varied by $18 \%$ of total image size), the direction predicted by the network was orthogonal to correct cyclone movement direction. This result probably reflects the complex training situation that arose when individual features were allowed to move outside the receptive field of individual feature detectors.

The present study is a proof of the concept and is meant to demonstrate the applicability of neural network techniques for cyclone track prediction. This work constitutes a first step towards fully automated interpretation of satellite images for the purpose of forecasting a cyclone's track and intensity. Among other things, the technique remains to be tested on higher resolution images of a wider variety of cyclones. Also, higher positional invariance may be desirable in real-world applications.

We see several directions for future work. First of all, it would be interesting to develop a scaled-up version of the network, and to compare its performance with the present 
network to see if higher resolution images contain additional useful information. Although our present training results indicate that low-resolution images are sufficient, we expect that some cases, especially at the early stages of cyclone development, may require recognition of subtle cloud patterns that may not be extractable from low-resolution satellite images.

Second, the kind of deep recurrent (bi-directionally connected) network that we have been using could be redesigned to also take into account other non-visual factors, such as the previous track of a cyclone, as well as sea-surface temperature and air pressure data. On the one hand, the inclusion of additional input layers would entail larger and more receiving layers, which in turn could slow the network simulations considerably. On the other hand, supplementary input would impose additional constraints on the activation states of the network. Provided that the constraints are intrinsically compatible, they could improve activation settling in the network, and could boost learning performance.

\section{References}

Barsi, A., Heipke, C., 2003a. Artificial neural networks for the detection of road junctions in aerial images. International Archives of Photogrammetry, Remote Sensing and Spatial Information Sciences 34 (Part 3/W8), 113-118.

Barsi, A., Heipke, C., 2003b. Detecting road junctions by artificial neural networks. Remote Sensing and Data Fusion over Urban Areas, Proc. 2nd GRSS/ISPRS Joint Workshop on Remote Sensing and Data Fusion over Urban Areas (URBAN 2003), Berlin, 22-23 May 2003, pp. 129-132.

Brad, R., Letia, I.A., 2002a. Extracting cloud motion from satellite image sequences, Proc. Seventh International Conference on Control, Automation, Robotics and Vision (ICARCV'2002), Singapore, 2-5 December 2002, pp. 1303-1307.

Brad, R., and Letia, I.A., 2002b. Cloud motion detection from infrared satellite images, Proc. Second International Conference on Image and Graphics (ICIG 2002), China, 1618 August 2002, 4875, pp 408-412.

Bureau of Meteorology Research Centre, 2006. Global guide to Tropical Cyclone forecasting, Australia, http://www.bom.gov.au/bmrc/pubs/tcguide/globa_guide_intro.htm (Accessed January 12, 2007).

Cerveny, R. S., Newman, L. E., 2000. Climatological relationships between tropical cyclones and rainfall. Monthly Weather Review 128 (9), 3329-3336.

Creem, S. H., Proffitt, D. R., 2001. Defining the cortical visual systems: "What", "Where", and "How". Acta Psychologica 107 (1-3), 43-68.

Dvorak, V.F., 1975. Tropical cyclone intensity analysis and forecasting from satellite imagery. Monthly Weather Review 103 (5), 420-430.

Felleman, D. J., Van Essen, D. C., 1991. Distributed hierarchical processing in the primate cerebral cortex. Cerebral Cortex 1, 1-47.

Fett, R.W., Brand, S., 1975. Tropical cyclone movement forecasts based on observations from satellites. Journal of Applied Meteorology 14 (4), 452-465.

Fukushima, K., Miyake, S., 1982. Neocognitron: A new algorithm for pattern recognition tolerant of deformations and shifts in position. Pattern Recognition 15 (6), 455-469. 
Goodale, M. A., Milner, A. D., 1992. Separate visual pathways for perception and action. Trends in Neuroscience 15 (1), 20-25.

Hong, Y., Hsu, K.-L., Sorooshian, S., Gao, X., 2004. Precipitation estimation from remotely sensed imagery using an artificial neural network cloud classification system. Journal of Applied Meteorology 43 (12), 1834-1852.

Jang, J.-D., Viau, A. A., Anctil, F., and Bartholomé, E., 2006. Neural network application for cloud detection in SPOT vegetation images. International Journal of Remote Sensing 27 (3-4), 719-736.

Kishtawal, C. M., Patadia F., Singh R., Basu S., Narayanan M. S., and Joshi P. C., 2005. Automatic estimation of tropical cyclone intensity using multi-channel TMI data: A genetic algorithm approach. Geophysical Research Letters 32 (11), L11804.1-L11804.5.

Knaff, J. A., Zehr, R. M., Goldberg, M. D., 2000. An example of temperature structure differences in two cyclone systems derived from the advanced microwave sounder unit. Weather and Forecasting 15 (4), 476-483.

Kossin J., 2003. A user's guide to the UW-CIMSS Tropical Cyclone Intensity Estimation (TIE) Model. Cooperative Institute for Meteorological Satellite Studies (CIMSS), University of Wisconsin, Madison, WI.

Kosslyn, S. M., 1994. Image and Brain: The resolution of the imagery debate. MIT Press, Cambridge, MA, pp. 53-79.

Lau, N. C., Crane, M. W., 1997. Comparing satellite and surface observations of cloud patterns in synoptic-scale circulation systems. Monthly Weather Review 125 (12), 3172-3189.

Lajoie, F. A., 1976. On the direction of movement of tropical cyclones.Australian Meteorological Magazine 24, 95-104.

Marshall, J. F. L., Leslie, L. M., Abbey Jr. R. F., Qi, L., 2002. Tropical cyclone track and intensity prediction: The generation and assimilation of high-density, satellite-derived data. Meteorology and Atmospheric Physics 80 (1-4), 43-57.

McBride, J.L., Holland, G.J., 1987. Tropical-Cyclone forecasting: A worldwide summery of techniques and verification statistics. Bulletin of the American Meteorological Society 68 (10), 1230-1238.

McClelland, J. L., 1993. The GRAIN model: a framework for modeling the dynamics of information processing. In: Meyer, D. E. and Kornblum, S., (Eds): Attention and Performance XIV: Synergies in Experimental Psychology, Artifical Intelligence, and Cognitive Neuroscience, MIT Press, Cambridge, MA, pp. 655-688.

Mutch, J., Lowe, D. G., 2008. Object class recognition and localization using sparse features with limited receptive fields. International Journal of Computer Vision 80 (1), 45-57.

Naval Research Laboratory, 1999. Tropical Cyclone Forecasters Reference Guide, http://www.nrlmry.navy.mil/ chu/ (Accessed January 13, 2007).

O'Reilly, R. C., Munakata, Y., 2000. Computational explorations in cognitive neuroscience: Understanding the mind by simulating the brain. MIT Press, Cambridge, MA, pp. 46-177. 
O'Reilly, R. C., 1998. Six principles for biologically based computational models of cortical processing. Trends in Cognitive Sciences 2 (11), 455-462.

Rivolta, G., Marzano, F.S., Coppola, E., Verdecchia, M., 2006. Artificial neural-network technique for precipitation nowcasting from satellite imagery. Advances in Geosciences 7, 97-103.

Spratling, M. W., 2005. Learning viewpoint invariant perceptual representations from cluttered images. IEEE Transactions on Pattern Analysis and Machine Intelligence 27 (5), 753-761

Mishkin, M., Ungerleider, L.G., Macko, K.A., 1983. Object vision and spatial vision: Two cortical pathways. Trends in Neurosciences 6 (10), 414-417.

Velden, C.S., Olander, T.L., Zehr, R.M., 1998. Development of an objective scheme to estimate tropical cyclone intensity from digital geostationary satellite infrared imagery, weather and forecasting 13 (1), 172-186.

Wells, F. H., 1987. Tropical cyclone intensity estimates using satellite data: The early years, Proc. 26th Conference on Hurricanes and Tropical Meteorology, Miami, FL, 2-7 May 2004, pp. 1-2. 


\section{Appendix B}

\section{TC signaling system for the maritime ports}

To convey danger levels associated with the approaching TC in the disseminated warnings, the Bangladesh Meteorological Department uses an eleven-point signaling system (ranging from low to high danger level) for the maritime ports. 


\section{The current TC signaling system for the maritime ports}

\begin{tabular}{|c|c|c|}
\hline $\begin{array}{c}\text { Signal } \\
\text { number }\end{array}$ & Signals & Explanations \\
\hline 1 & $\begin{array}{l}\text { Distant cautionary } \\
\text { signal number-I }\end{array}$ & $\begin{array}{l}\text { There is a region of squally weather (wind } \\
\text { speed of } 61 \mathrm{~km} / \mathrm{h} \text { ) in the distant sea where a } \\
\text { storm may form. }\end{array}$ \\
\hline 2 & $\begin{array}{l}\text { Distant warning signal } \\
\text { number-II }\end{array}$ & $\begin{array}{l}\text { A storm (wind speed of } 62-88 \mathrm{~km} / \mathrm{h} \text { ) has } \\
\text { formed in the distant deep sea. Ships may fall } \\
\text { into danger if they leave harbor. }\end{array}$ \\
\hline 3 & $\begin{array}{l}\text { Local cautionary } \\
\text { signal number-III }\end{array}$ & $\begin{array}{l}\text { The port is threatened by squally weather } \\
\text { (wind speed of } 40-50 \mathrm{~km} / \mathrm{h} \text { ). }\end{array}$ \\
\hline 4 & $\begin{array}{l}\text { Local warning signal } \\
\text { number-IV }\end{array}$ & $\begin{array}{l}\text { The port is threatened by a storm (wind speed } \\
\text { of } 51-61 \mathrm{~km} / \mathrm{h} \text { ) but it does not appear that the } \\
\text { danger is yet sufficiently great to justify } \\
\text { extreme precautionary measures. }\end{array}$ \\
\hline 5 & $\begin{array}{l}\text { Danger signal } \\
\text { number-V }\end{array}$ & $\begin{array}{l}\text { The port will experience severe weather from a } \\
\text { storm of slight or moderate intensity (wind } \\
\text { speed of } 62-88 \mathrm{~km} / \mathrm{h} \text { ) that is expected to cross } \\
\text { the coast to the south of Chittagong port or } \\
\text { Cox's Bazar port and to the east of Mongla } \\
\text { port. }\end{array}$ \\
\hline 6 & $\begin{array}{l}\text { Danger signal } \\
\text { number-VI }\end{array}$ & $\begin{array}{l}\text { The port will experience severe weather from a } \\
\text { storm of slight or moderate intensity (wind } \\
\text { speed of } 62-88 \mathrm{~km} / \mathrm{h} \text { ) that is expected to cross } \\
\text { the coast to the north of the port of Chittagong } \\
\text { or Cox's Bazar and to the west of the port of } \\
\text { Mongla. }\end{array}$ \\
\hline 7 & $\begin{array}{l}\text { Danger signal } \\
\text { number-VII }\end{array}$ & $\begin{array}{l}\text { The port will experience severe weather from a } \\
\text { storm of light or moderate intensity (wind } \\
\text { speed of } 62-88 \mathrm{~km} / \mathrm{h} \text { ) that is expected to cross } \\
\text { over or near the port. }\end{array}$ \\
\hline 8 & $\begin{array}{l}\text { Great danger signal } \\
\text { number-VIII }\end{array}$ & $\begin{array}{l}\text { The port will experience severe weather from a } \\
\text { storm of great intensity (wind speed of } 89 \\
\mathrm{~km} / \mathrm{h} \text { or more) that is expected to cross the } \\
\text { coast to the south of the port of Chittagong or } \\
\text { Cox's Bazar and to the east of the port of } \\
\text { Mongal. }\end{array}$ \\
\hline 9 & $\begin{array}{l}\text { Great danger signal } \\
\text { number-IX }\end{array}$ & $\begin{array}{l}\text { The port will experience severe weather from a } \\
\text { storm of great intensity (wind speed of } 89 \\
\mathrm{~km} / \mathrm{h} \text { or more) that is expected to cross the } \\
\text { coast to the north or the port of Chittagong or }\end{array}$ \\
\hline
\end{tabular}




\begin{tabular}{cll}
\hline $\begin{array}{c}\text { Signal } \\
\text { number }\end{array}$ & \multicolumn{1}{c}{ Signals } & \multicolumn{1}{c}{ Explanations } \\
\hline & & $\begin{array}{l}\text { Cox's Bazar and to the west of the port of } \\
\text { Mongla. }\end{array}$ \\
\hline 10 & $\begin{array}{l}\text { Great danger signal } \\
\text { number-X }\end{array}$ & $\begin{array}{l}\text { The port will experience severe weather from a } \\
\text { storm of great intensity (wind speed of } 89 \\
\mathrm{~km} / \mathrm{h} \text { or more) that is expected to cross over or } \\
\text { near the port. }\end{array}$ \\
\hline 11 & $\begin{array}{l}\text { Failure of } \\
\text { communication-XI }\end{array}$ & $\begin{array}{l}\text { Communications with the Storm Warning } \\
\text { Centre have broken down and local officers } \\
\text { consider that a devastating cyclone is } \\
\text { following. }\end{array}$ \\
\hline
\end{tabular}




\section{Appendix C}

\section{TC signaling system for the river ports}

To convey danger levels associated with the approaching TC in the disseminated warnings, The Bangladesh Meteorological Department uses a four-point signaling system for the river ports. Signal number 1 in this system represents low danger level, whereas signal number 4 represents high danger level. 


\section{The current TC signaling system for the river ports}

\begin{tabular}{cll}
\hline $\begin{array}{c}\text { Signal } \\
\text { number }\end{array}$ & \multicolumn{1}{c}{ Signals } & \multicolumn{1}{c}{ Explanations } \\
\hline 1 & $\begin{array}{l}\text { Cautionary signal } \\
\text { number-I }\end{array}$ & $\begin{array}{l}\text { The area is threatened by squally winds (wind } \\
\text { speed of } 60 \mathrm{~km} / \mathrm{h} \text { ) of transient nature. This } \\
\text { signal is also hoisted during nor'westers. }\end{array}$ \\
\hline 2 & Warning signal & $\begin{array}{l}\text { A storm (wind speed of } 61 \mathrm{~km} / \mathrm{h} \text { ) or a } \\
\text { nor'wester (wind speed } 61 \mathrm{~km} / \mathrm{h} \text { or more) is } \\
\text { likely to strike the area }(\text { Vessels of } 65 \text { feet and } \\
\text { under in length are to seek shelter } \\
\text { immediately. }\end{array}$ \\
\hline 3 & $\begin{array}{l}\text { Danger signal } \\
\text { number-III }\end{array}$ & $\begin{array}{l}\text { A storm (wind speed of } 62-88 \mathrm{~km} / \mathrm{h} \text { or more) is } \\
\text { likely to strike the area soon (all vessels will } \\
\text { seek shelter immediately). }\end{array}$ \\
\hline 4 & $\begin{array}{l}\text { Great danger } \\
\text { signal number-IV }\end{array}$ & $\begin{array}{l}\text { A violent storm (wind speed of } 89 \mathrm{~km} / \mathrm{h} \text { or } \\
\text { more) will strike the area soon }(\text { all } \mathrm{Vessels} \text { will } \\
\text { take shelter immediately). }\end{array}$ \\
\hline
\end{tabular}




\title{
Appendix D
}

\section{Questions used for the in-depth interview}

\begin{abstract}
A collection of structured and open-ended questions was used for the in-depth interview among the meteorologists at the Bangladesh Meteorological Department. All the questions used for the interview were organized under two sections. Questions in the first section were used to collect information about the participating meteorologists themselves and the second section was devoted to elicit their views on the cyclone early warning system, which is currently in use.
\end{abstract}




\section{Meteorologists' views on the current cyclone forecasting and warning system at the Bangladesh meteorological department}

\section{Section 1 (Respondent details)}

\begin{tabular}{|c|c|c|c|}
\hline \multicolumn{4}{|l|}{ Name } \\
\hline Age & & Sex & $\square$ Male $\square$ Female \\
\hline \multicolumn{4}{|c|}{ Responsibility } \\
\hline $\begin{array}{l}\text { Educational } \\
\text { background }\end{array}$ & $\square$ Physics & $\square$ Mathematics & $\begin{array}{l}\square \text { Meteorology } \\
\text { Other }\end{array}$ \\
\hline Started job in & & & \\
\hline
\end{tabular}

\section{Section 2 (Cyclone forecasting and warning)}

1) Which technique/techniques are currently in use at BMD to produce forecast for Tropical Cyclones (TCs)?

2) Data used by BMD for forecasting TCs

\begin{tabular}{l|l|l|l|l|l}
\hline \multicolumn{2}{c|}{ Ground-based observation } & \multicolumn{3}{c}{ Satellite observation } \\
\hline \multicolumn{1}{c|}{ Data type } & $\begin{array}{c}\text { Freely } \\
\text { available } \\
(\mathrm{Y} / \mathrm{N})\end{array}$ & $\begin{array}{c}\text { Used for } \\
\text { TC forecast } \\
(\mathrm{Y} / \mathrm{N})\end{array}$ & $\begin{array}{c}\text { Data type } \\
\text { Fvailable } \\
(\mathrm{Y} / \mathrm{N}) \text { and } \\
\text { satellite }\end{array}$ & $\begin{array}{c}\text { Used for } \\
\text { TC } \\
\text { forecast } \\
(\mathrm{Y} / \mathrm{N})\end{array}$ \\
\hline Surface & & & Infrared image & & \\
\hline Surface humidity & & & Sea level pressure & & \\
\hline Rainfall & & & Sea surface & & \\
\hline $\begin{array}{l}\text { Surface pressure } \\
\text { speed }\end{array}$ & & & Vertical wind & & \\
\hline $\begin{array}{l}\text { Surface wind } \\
\text { direction }\end{array}$ & & & $\begin{array}{l}\text { Multiple level } \\
\text { temperature }\end{array}$ & & \\
\hline Radar data & & $\begin{array}{l}\text { Visible channel } \\
\text { image }\end{array}$ & & \\
\hline & & & Wind speed & & \\
\hline & & & Wind direction & & \\
\hline
\end{tabular}

3) TC forecasting technique detail (use the following table if more than one technique is used)

\begin{tabular}{l|l|l|l}
\hline Name & \multicolumn{3}{|c}{} \\
\hline Type & $\square$ Numerical & $\square$ Statistical & $\square$ Other......... \\
\hline
\end{tabular}




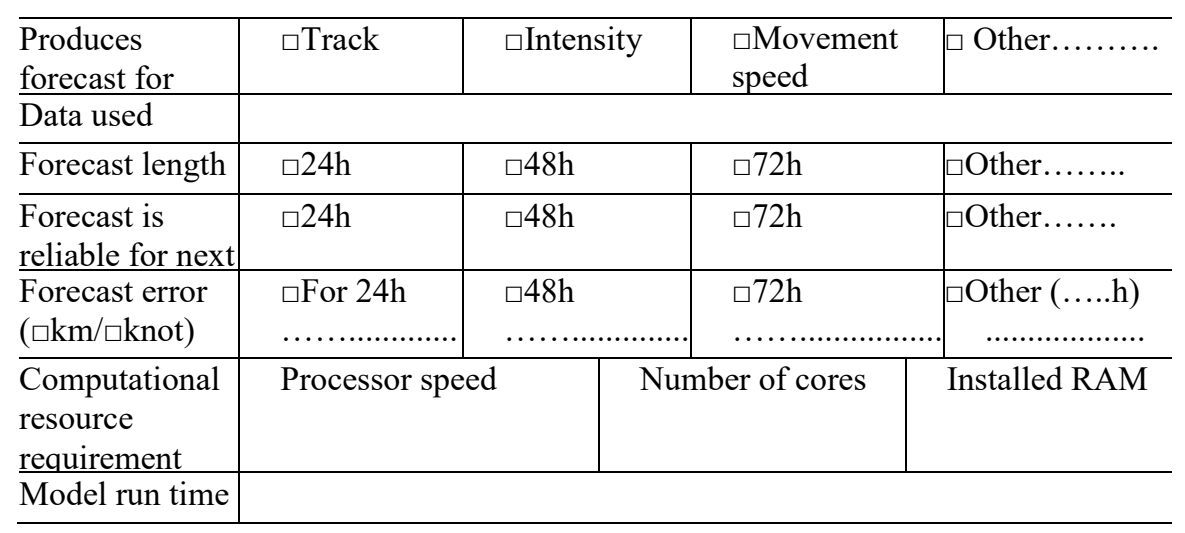

4) Have there been any changes in TC forecasting techniques during the last decade ( $\square$ Yes/ $\square$ No)? If yes please state reasons and effects of the changes

\begin{tabular}{c|c|c|c}
\hline Change & $\begin{array}{c}\text { Time of } \\
\text { change }\end{array}$ & Reason of change & Effect of the change \\
\hline & & & \\
\hline & & & \\
\hline
\end{tabular}

5) Possible ways to improve the precision of TC forecast in future

a) Using new data (Please describe)

b) Modifying the existing technique (Please describe)

c) Using better techniques (Please describe)

d) Others

6) Why are the warning messages issued for maritime and river ports and not for other places where cyclones will make a landfall?

a) Due to the geographical locations of the maritime and river ports

b) Due to the pattern of historical cyclone tracks

c) Due to the shape and physiography of the coast

d) The techniques currently in use are not capable of producing track forecast precisely, so mentioning only the ports in the warning message is a simplified resolution of the problem

e) Other reasons for not identifying other places and cities as threatened

7) Does the content of the warning message vary according to the danger level $(\square \mathrm{Yes} / \square \mathrm{No})$ ? Please describe the warning message formulation process.

8) How are the TC warning messages delivered (using which media, e.g., TV, radio, text messages)? 
9) How precise and reliable are the current warning messages?

[1] [2] [3] [4] [5] [6]

(Never correct) (Always correct)

10) Possible ways to improve the precision and reliability of warning message in future

a) Forecast TCs with better accuracy (Please describe)

b) Increase the efficiency of warning message delivery system (Please describe)

c) Others

11) Does the BMD receive cyclone forecasts from other meteorological offices or organizations? ( $\square$ Yes/ $\square$ No)? If yes please state the names
$\checkmark$ regional specialized meteorological center
口 UKMET
$\square$ IMD
$\square$ Others...
$\square$ JMA

12) BMD delivers cyclone warning messages based on:
$\square$ own forecast
$\square$ received forecast
$\checkmark$ combined (own plus received) forecast

13) What are the future plans of BMD regarding TC forecasting and warning system? 


\section{Appendix E}

\section{Questionnaire used for the survey}

The questionnaire used to elicit respondents' views on the current cyclone early warning system consisted of two parts. The first part contained structured questions, which were used to collect socio-economic information from the respondents, whereas the second part of the questionnaire contained multiple-choice questions and elicited respondents' views on the current cyclone early warning system. 


\section{Residents' views on the current cyclone early warning system-the case of cyclone Sidr and Mahasen in Bangladesh}

Part 1 (Respondent detail):

\begin{tabular}{|c|c|c|c|c|}
\hline Name & & & & \\
\hline Address & Village: & \multicolumn{2}{|c|}{ Union: } & Thana: \\
\hline $\begin{array}{l}\text { Location of } \\
\text { respondent's } \\
\text { home }\end{array}$ & \multicolumn{2}{|l|}{ Lat: } & \multicolumn{2}{|l|}{ Long: } \\
\hline Age & \multicolumn{2}{|c|}{\begin{tabular}{c|c} 
& Sex \\
$\square$ Service $\square$ Business $\square \mathrm{F}$
\end{tabular}} & \multicolumn{2}{|c|}{$\square$ Male $\square$ Female } \\
\hline Occupation & \multicolumn{4}{|c|}{$\begin{array}{l}\square \text { Service } \square \text { Business } \square \text { Farming } \square \text { Fishing } \\
\square \text { Other............... }\end{array}$} \\
\hline \multicolumn{5}{|l|}{ Income (approx.) } \\
\hline Education & \multicolumn{4}{|c|}{$\begin{array}{l}\square \text { Illiterate } \square \text { Primary school } \square \text { Secondary school } \\
\square \text { Higher education }\end{array}$} \\
\hline Family size & \multicolumn{3}{|c|}{ Number of dependents } & \\
\hline $\begin{array}{l}\text { Condition of } \\
\text { surrounding roads }\end{array}$ & \multicolumn{4}{|c|}{$\begin{array}{l}\square \text { Tar built } \square \text { Concrete } \square \text { Semi-concrete } \square \text { Brick-built } \\
\square \text { Mud-built }\end{array}$} \\
\hline $\begin{array}{l}\text { Type of housing } \\
\text { (ability to } \\
\text { withstand storm) }\end{array}$ & $\begin{array}{l}\text { Katcha (floor of } \\
\text { mud, wall of } \\
\text { wood/tin/hay, roof } \\
\text { of tin/hay) }\end{array}$ & \multicolumn{2}{|c|}{$\begin{array}{l}\text { Semi pacca (floor } \\
\text { of concrete, wall of } \\
\text { wood/tin, roof of } \\
\text { tin) }\end{array}$} & $\begin{array}{l}\text { Pacca (floor and } \\
\text { wall of concrete, } \\
\text { roof of } \\
\text { tin/concrete) }\end{array}$ \\
\hline
\end{tabular}

Part 2 (Receiving of warning message, interpretation, and response)

1) Have you received any cyclone warning during Sidr ( $\square \mathrm{Yes} / \square$ No) and Mahasen $(\square \mathrm{Yes} / \square \mathrm{No})$ ?

2) If yes, from where did you receive the cyclone warning

口electronic media (radio, TV, sms) $\quad$ newspaper

口signal flag $\quad$ 口megaphone

口other.

3) From which media you prefer to get warning messages and why?

\begin{tabular}{|c|c|}
\hline Media & Reasons of the preference \\
\hline 口electronic media (radio, TV, sms) & $\square$ don’t have mobile phone \\
\hline$\square$ newspaper & $\square$ cannot hear megaphone because live too far \\
\hline$\square$ signal flag & 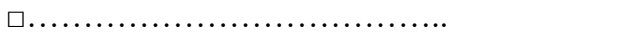 \\
\hline$\square$ megaphone & 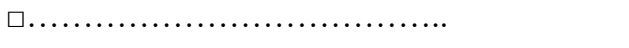 \\
\hline$\square$ other................... & 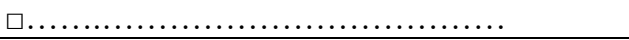 \\
\hline
\end{tabular}

4) Warning message from which media is easy to understand for you
$\square$ electronic media (radio, TV, sms)
$\square$ newspaper
$\square$ signal flag
$\square$ megaphone 
$\square$ other.

5) Do you have access to any cyclone shelter ( $\square$ Yes $/ \square$ No)? If yes please mention the name.... and distance. of the shelter from your home.

6) Please listen/read the warning messages I am going to read/ I am going to supply (Cyclone Sidr warning)

Warning message disseminated on 12 November 2007

What information do you get from the warning message?

a) Current position of the approaching cyclone

b) Forecasted position of the approaching cyclone

c) Danger level signal

d) Areas likely to be affected

e) Approximate time of strike

f) Maximum wind speed or intensity

g) Approximate height of the storm surge

h) Evacuation guidance

i) Safety guidance for the fishing boats

j) Other

Warning message disseminated on 15 November 2007

Which information do you get from the warning message?

a) Current position of the approaching cyclone

b) Forecasted position of the approaching cyclone

c) Danger level signal

d) Areas likely to be affected

e) Approximate time of strike

f) Maximum wind speed or intensity

g) Approximate height of the storm surge

h) Evacuation guidance

i) Safety guidance for the fishing boats

j) Other.

7) Are there any differences between the warning messages you just listened to $(\square$ Yes/ロNo)? If yes, please mention in which respect the warning messages are different?

a) Current position of the approaching cyclone

b) Forecasted position of the approaching cyclone

c) Danger level signal

d) Areas likely to be affected

e) Approximate time of strike

f) Maximum wind speed or intensity

g) Approximate height of the storm surge

h) Evacuation guidance

i) Safety guidance for the fishing boats

j) Other....

8) Did you go to cyclone shelter/other safe place after receiving the warning during Sidr ( $\square$ Yes/ $\square$ No)? If no describe the reasons 


\begin{tabular}{l|l}
\hline$\square$ did not trust the warning message & $\begin{array}{l}\square \text { did not understand the instructions } \\
\text { in the warning message }\end{array}$ \\
\hline $\begin{array}{l}\square \text { according to the warning, the } \\
\text { cyclone would not strike at this part } \\
\text { of the coast so I thought that the } \\
\text { danger was low }\end{array}$ & $\begin{array}{l}\square \text { I thought that the accommodation } \\
\text { capacity of the shelter is not enough }\end{array}$ \\
\hline $\begin{array}{l}\square \text { the nearest shelter is too far } \\
\square \text { afraid of residential burglary } \\
\text { condition }\end{array}$ & $\begin{array}{l}\square \text { local communication system is } \\
\text { poor }\end{array}$ \\
\hline $\begin{array}{l}\square \text { home is strong so did not feel the } \\
\text { necessity to go to shelter }\end{array}$ & $\square$ others \\
\hline
\end{tabular}

9) Please listen/read the warning messages I am going to read/ I am going to supply (Cyclone Mahasen warning)

Warning message disseminated on 12 May 2013

Which information do you get from this warning message?

a) Current position of the approaching cyclone

b) Forecasted position of the approaching cyclone

c) Danger level signal

d) Areas likely to be affected

e) Approximate time of strike

f) Maximum wind speed or intensity

g) Approximate height of the storm surge

h) Evacuation guidance

i) Safety guidance for the fishing boats

j) Other.

Warning message disseminated on 16 May 2013

Which information do you get from this warning message?

a) Current position of the approaching cyclone

b) Forecasted position of the approaching cyclone

c) Danger level signal

d) Areas likely to be affected

e) Approximate time of strike

f) Maximum wind speed or intensity

g) Approximate height of the storm surge

h) Evacuation guidance

i) Safety guidance for the fishing boats

j) Other.

10) Are there any differences between the warning messages you just listened to ( $\square$ Yes/ $\square$ No)? If yes then please mention in which respect the warning messages are different?

a) Current position of the approaching cyclone

b) Forecasted position of the approaching cyclone

c) Danger level signal

d) Areas likely to be affected

e) Approximate time of strike 
f) Maximum wind speed or intensity

g) Approximate height of the storm surge

h) Evacuation guidance

i) Safety guidance for the fishing boats

j) Other.

11) Did you go to cyclone shelter/other safe place after receiving the warning during Mahasen ( $\square$ Yes/ $\square$ No)? If no describe the reasons

\begin{tabular}{l|l}
\hline$\square$ did not trust the warning message & $\begin{array}{l}\square \text { did not understand the instructions } \\
\text { in the warning message }\end{array}$ \\
\hline $\begin{array}{l}\square \text { according to the warning, the } \\
\text { cyclone would not strike at this } \\
\text { part of the coast so I thought that } \\
\text { the danger was low }\end{array}$ & $\begin{array}{l}\square \text { thought that the accommodation } \\
\text { capacity of the shelter is not enough }\end{array}$ \\
\hline $\begin{array}{l}\square \text { the nearest shelter is too far } \\
\square \text { atraid of residential burglary } \\
\text { condition }\end{array}$ & $\begin{array}{l}\square \text { local communication system is } \\
\text { poor }\end{array}$ \\
\hline $\begin{array}{l}\square \text { home is strong so did not feel the } \\
\text { necessity to go to shelter }\end{array}$ & $\square$ others \\
\hline
\end{tabular}

12) To what extent are you satisfied with the warning messages you received during cyclone Sidr and Mahasen ( $\square$ very high $\square$ high $\square$ moderate $\square$ low $\square$ very low)? Describe the reason:
a) Reliability/accuracy of the warning message
b) Timeliness of the warning message
c) Understandability of the warning message
d) Other.

13) In which way should the warning messages be improved for future cyclones?

a) Including good description of the threat (certainty/severity/immediacy/duration)

b) Guidance on protective action

c) Where I can get further information about the cyclone

d) Other.... 


\section{Appendix F}

\section{Per-recorded warning messages}

To get insight into respondents' ability to understand and interpret the information contained in the disseminated warnings, four pre-recorded warning messages (two for each of the two TCs Sidr and Mahasen) were used in the questionnaire survey. All the 200 respondents listened to these pre-recorded warnings during the survey and they answered the multiple-choice questions: 6, 7, 9, and 10 in Appendix E after listening. 


\title{
The four pre-recorded warning messages (two for each of the two TCs: Sidr and Mahasen) used in the questionnaire survey
}

\author{
Special weather bulletin: sl. no: 03 (three), date: 12-11-2007
}

The depression over south east bay and adjoining area remained practically stationary, intensified into a deep depression over the same area and was centered at 06 am today $\left(12^{\text {th }}\right.$ November 2007) about 1360 kilometers south of Chittagong port, 1270 kilometers south of cox's bazar port and 1385 kilometers south-southeast of Mongla port (near lat $10.0^{\circ} \mathrm{N}$ and long $91.5^{\circ} \mathrm{E}$ ). It is likely to intensify further and move in a northwesterly direction.

Maximum sustained wind speed within 48 kilometers of the depression center is about $50 \mathrm{~km} / \mathrm{h}$ rising to $60 \mathrm{~km} / \mathrm{h}$ in gusts/squalls. Sea will remain rough.

Maritime ports of Chittagong, cox's bazar and Mongla have been advised to keep hoisted distant cautionary signal number one (repeat) one.

All fishing boats and trawlers over north bay have been advised to come close to the coast and proceed with caution till further notice. They are also advised not to venture into the deep sea.

\section{Special weather bulletin: sl. no. 20 (twenty), date: 15-11-2007}

The severe cyclonic storm "Sidr" (ECP $952 \mathrm{hpa}$ ) with a core of hurricane winds over east central bay and adjoining area moved northwards and was centered at midnight last night about 705 kilometers south-southwest of Chittagong port, 625 kilometers south-southwest of cox's bazar port and 650 kilometers south of Mongla port (near lat $16.5^{\circ} \mathrm{N} \&$ long $89.2^{\circ}$ E). It is likely to intensify further and move in a northerly direction and cross KhulnaBarisal coast by noon today $\left(15^{\text {th }}\right.$ November 2007).

Maximum sustained wind speed within 74 kilometers of the storm center is about 190 $\mathrm{km} / \mathrm{h}$ rising to $210 \mathrm{~km} / \mathrm{h}$ in gusts/squalls. Sea will remain very high.

Maritime port of Mongla has been advised to keep hoisted great danger signal no. ten (repeat) ten. the coastal districts of Bhola, Barisal, Patuakhali, Borguna, Pirozpur, Jhalokathi, Bagerhat, Khulna, Satkhira and their offshore islands and chars will remain under great danger signal no. ten (repeat) ten.

Maritime ports of Chittagong and cox's bazar have been advised to keep hoisted great danger singal no. nine (r) nine. The coastal districts of Cox's bazar, Chittagong, Noakhali, Feni, Laxmipur, Chandpur and their offshore islands and chars will remain under great danger signal no. nine (repeat) nine.

Under the influence of the storm, the coastal districts of Cox's bazar, Chittagong, Noakhali, Feni, Laxmipur, Bhola, Barisal, Patuakhali, Borguna, Chandpur, Pirozpur, Jhalokathi, Bagerhat, Khulna, Satkhira and their offshore islands and chars are likely to experience squally wind speed $120-150 \mathrm{~km} / \mathrm{h}$ or more from morning today which will gradually increase up to $210 \mathrm{~km} / \mathrm{h}$ with the passage of the storm.

The low-lying areas of the coastal districts of Cox's bazar, Chittagong, Noakhali, Feni, Laxmipur, Bhola, Barisal, Patuakhali, Borguna, Chandpur, Pirozpur, Jhalokathi, Bagerhat, 
Khulna, Satkhira and their offshore islands and chars are likely to be inundated by storm surge of height 12-15 feet above normal astronomical tide. All fishing boats and trawlers over north bay must remain in shelter till further notice.

\section{Special weather bulletin: sl. no. 05 (five), date: 12-05-2013}

The cyclonic storm "Mahasen" (with ECP $998 \mathrm{hpa}$ ) over southeast bay and adjoining area moved northwestwards over the same area and was centered at midnight last night $\left(11^{\text {th }}\right.$ May 2013) about 1555 kilometers south southwest of Chittagong port, 1465 kilometers south southwest of cox's bazar port and 1535 kilometers south of Mongla port (near lat $8.5^{\circ}$ $\mathrm{N}$ and long $89.2^{\circ} \mathrm{E}$ ). It is likely to intensify further and move in a north northwesterly direction.

Maximum sustained wind speed within 54 kilometers of the storm center is about 62 $\mathrm{km} / \mathrm{h}$ rising to $88 \mathrm{~km} / \mathrm{h}$ in gusts/ squalls. Sea will remain very rough near the storm center.

Steep pressure gradient persists over north bay. Under its influence, squally weather may affect north bay, adjoining coastal area of Bangladesh and the maritime ports.

Maritime ports of Chittagong, cox's bazar and Mongla have been advised to keep hoisted local cautionary signal no. three (repeat) three.

All fishing boats and trawlers over north bay have been advised to remain close to the coast and proceed with caution. They are also advised not to venture into the deep sea until further notice.

\section{Special weather bulletin: sl. no. 29 (twenty nine), date: 16-05-2013}

The cyclonic storm "Mahasen" (with ECP 990 hpa) over north bay and adjoining west central bay moved slightly north-northeastwards and now lies over the same area and was centered at midnight last night (the 15 may 2013) about 555 kilometers southwest of Chittagong port, 500 kilometers southwest of cox's bazar port and 445 kilometers south southwest of Mongla port (near lat $18.5^{\circ} \mathrm{N}$ and long $88.5^{\circ} \mathrm{E}$ ).

It is likely to intensify further and move in a north-northeasterly direction and may cross between Patuakhali (Khepupara)-Teknaf coast near Chittagong by noon of $16^{\text {th }}$ May 2013.

Maximum sustained wind speed within 54 kilometers of the storm center is about 62 $\mathrm{km} / \mathrm{h}$ rising to $90 \mathrm{~km} / \mathrm{h}$ in gusts/ squalls. Sea will remain very rough near the storm center.

Maritime ports of Chittagong and cox's bazar have been advised to keep hoisted danger signal number seven (repeat) seven.

The coastal districts of Cox's bazar, Chittagong, Noakhali, Laxmipur, Feni, Chandpur, Bhola, Borguna, Patuakhali, Barisal and their offshore islands and chars will come under danger signal number seven (repeat) seven.

Maritime port of mongla has been advised to keep hoisted danger signal number five (repeat) five. The coastal districts of Pirozpur, Jhalokathi, Bagherhat, Khulna, Satkhira and their offshore islands and chars will come under danger signal number five (repeat) five.

Under the influence of the storm the low-lying areas of the coastal districts of Cox's bazar, Chittagong, Noakhali, Laxmipur, Feni, Chandpur, Borguna, Bhola, Patuakhali, Barisal, Pirozpur, Jhalokathi, Bagherhat, Khulna, Satkhira and their offshore islands and chars are likely to be inundated by storm surge of 8-10 feet height above normal astronomical tide. 
The coastal districts of Cox's bazar, Chittagong, Noakhali, Laxmipur, Feni, Chandpur, Borguna, Patuakhali, Barisal, Bhola, Pirozpur, and their offshore islands and chars are likely to experience wind speed up to $90-100 \mathrm{~km} / \mathrm{h}$ in gusts/ squalls with heavy to very falls during the passage of the storm.

The coastal districts of Jhalokathi, Bagerhat, Khulna, Satkhira and their offshore islands and chars are likely to experience wind speed up to $80-90 \mathrm{~km} / \mathrm{h}$ in gusts/ squalls with heavy to very falls during the passage of the storm.

Due to very heavy rainfall, landslide may occur at places over the hilly regions of Chittagong division. All fishing boats, trawlers, and marine vessels over north bay have been advised to remain in shelter till further notice. 


\section{Appendix G}

\section{Generalized TC signaling system}

The Bangladesh Department of Disaster Management in cooperation with the Bangladesh Meteorological Department has recently designed an eight-point signaling system for TCs, which is to be used for both the maritime and the river ports. In this new system, all the eight signal numbers are to be used for the maritime ports and the last six signal numbers are to be used for the river ports. 


\section{Generalized eight-point TC signaling system for the maritime and the river ports}

\begin{tabular}{|c|c|c|}
\hline $\begin{array}{c}\text { Signal } \\
\text { number }\end{array}$ & Signals & Explanations \\
\hline 1 & $\begin{array}{l}\text { Distance } \\
\text { cautionary signal } \\
\text { number-I }\end{array}$ & $\begin{array}{l}\text { There is a region of squally weather in which a } \\
\text { storm may be forming (well-marked low or } \\
\text { depression) with surface winds up to } 61 \mathrm{~km} / \mathrm{h} \text {. } \\
(33 \mathrm{knots}) \text { ). }\end{array}$ \\
\hline 2 & $\begin{array}{l}\text { Distance warning } \\
\text { signal number-II }\end{array}$ & $\begin{array}{l}\text { A storm has formed (Cyclonic storm with } \\
\text { surface winds } 62-87 \mathrm{~km} / \mathrm{h} \text {. (34-47 knots)). }\end{array}$ \\
\hline 3 & $\begin{array}{l}\text { Local cautionary } \\
\text { signal number-III }\end{array}$ & $\begin{array}{l}\text { The port/area is threatened by squally weather } \\
\text { (cyclonic circulation with surface winds } 40-50 \\
\mathrm{~km} / \mathrm{h} \text {. ( } 22-27 \text { knots) or squalls due to } \\
\text { Nor'westers). }\end{array}$ \\
\hline 4 & $\begin{array}{l}\text { Local warning } \\
\text { signal number-IV }\end{array}$ & $\begin{array}{l}\text { The port/area is threatened by a storm, but it } \\
\text { does not appear that the danger is as yet } \\
\text { sufficiently great to justify extreme measures of } \\
\text { precaution (cyclonic circulation) with surface } \\
\text { winds } 51-61 \mathrm{~km} / \mathrm{h} \text {. (28-33 knots)). }\end{array}$ \\
\hline 5 & Danger Signal-VI & $\begin{array}{l}\text { The port/area will experience severe weather } \\
\text { from a cyclonic storm of moderate intensity } \\
\text { (Cyclonic storm with surface winds } 62-88 \\
\mathrm{~km} / \mathrm{h} .(34-47 \text { knots)). }\end{array}$ \\
\hline 6 & $\begin{array}{l}\text { Great Danger } \\
\text { Signal-IX }\end{array}$ & $\begin{array}{l}\text { The port will experience severe weather from a } \\
\text { storm of very great intensity (Severe cyclonic } \\
\text { storm with surface winds } 89-117 \mathrm{~km} / \mathrm{h} \text {. (48- } \\
63) \text { ). }\end{array}$ \\
\hline 7 & $\begin{array}{l}\text { Great Danger } \\
\text { Signal-IX }\end{array}$ & $\begin{array}{l}\text { The port will experience severe weather from a } \\
\text { storm of very great intensity (Severe cyclonic } \\
\text { storm with a core of Hurricane winds with } \\
\text { surface winds } 118-170 \mathrm{~km} / \mathrm{h} \text {. (64-119 knots)). }\end{array}$ \\
\hline 8 & $\begin{array}{l}\text { Great Danger } \\
\text { Signal-X }\end{array}$ & $\begin{array}{l}\text { The Port will experience severe weather from a } \\
\text { storm of very great intensity (Severe cyclonic } \\
\text { storm with a core of Hurricane winds with } \\
\text { surface winds } 171 \mathrm{~km} / \mathrm{h} \text { and above ( } 120 \text { knots } \\
\text { and above)). }\end{array}$ \\
\hline
\end{tabular}




\section{Dissertations}

\section{Linköping Studies in Science and Technology Linköping Studies in Arts and Science \\ Linköping Studies in Statistics \\ Linköpings Studies in Information Science}

Linköping Studies in Science and Technology

No 14 Anders Haraldsson: A Program Manipulation System Based on Partial Evaluation, 1977, ISBN 91-7372-1441.

No 17 Bengt Magnhagen: Probability Based Verification of Time Margins in Digital Designs, 1977, ISBN 917372-157-3.

No 18 Mats Cedwall: Semantisk analys av processbeskrivningar i naturligt språk, 1977, ISBN 91- 7372$168-9$.

No 22 Jaak Urmi: A Machine Independent LISP Compiler and its Implications for Ideal Hardware, 1978, ISBN 91-7372-188-3.

No 33 Tore Risch: Compilation of Multiple File Queries in a Meta-Database System 1978, ISBN 91- 7372-232-4.

No 51 Erland Jungert: Synthesizing Database Structures from a User Oriented Data Model, 1980, ISBN 917372-387-8.

No 54 Sture Hägglund: Contributions to the Development of Methods and Tools for Interactive Design of Applications Software, 1980, ISBN 91-7372-404-1.

No 55 Pär Emanuelson: Performance Enhancement in a Well-Structured Pattern Matcher through Partial Evaluation, 1980, ISBN 91-7372-403-3.

No 58 Bengt Johnsson, Bertil Andersson: The HumanComputer Interface in Commercial Systems, 1981, ISBN 91-7372-414-9.

No 69 H. Jan Komorowski: A Specification of an Abstract Prolog Machine and its Application to Partial Evaluation, 1981, ISBN 91-7372-479-3.

No 71 René Reboh: Knowledge Engineering Techniques and Tools for Expert Systems, 1981, ISBN 91-7372-489-0.

No 77 Östen Oskarsson: Mechanisms of Modifiability in large Software Systems, 1982, ISBN 91- 7372-527-7.

No 94 Hans Lunell: Code Generator Writing Systems, 1983, ISBN 91-7372-652-4.

No 97 Andrzej Lingas: Advances in Minimum Weight Triangulation, 1983, ISBN 91-7372-660-5.

No 109 Peter Fritzson: Towards a Distributed Programming Environment based on Incremental Compilation, 1984, ISBN 91-7372-801-2.

No 111 Erik Tengvald: The Design of Expert Planning Systems. An Experimental Operations Planning System for Turning, 1984, ISBN 91-7372-805-5.

No 155 Christos Levcopoulos: Heuristics for Minimum Decompositions of Polygons, 1987, ISBN 91-7870133-3.

No 165 James W. Goodwin: A Theory and System for NonMonotonic Reasoning, 1987, ISBN 91-7870-183-X.

No 170 Zebo Peng: A Formal Methodology for Automated Synthesis of VLSI Systems, 1987, ISBN 91-7870-2259.

No 174 Johan Fagerström: A Paradigm and System for Design of Distributed Systems, 1988, ISBN 91-7870301-8.

No 192 Dimiter Driankov: Towards a Many Valued Logic of Quantified Belief, 1988, ISBN 91-7870-374-3.
No 213 Lin Padgham: Non-Monotonic Inheritance for an Object Oriented Knowledge Base, 1989, ISBN 917870-485-5.

No 214 Tony Larsson: A Formal Hardware Description and Verification Method, 1989, ISBN 91-7870-517-7.

No 221 Michael Reinfrank: Fundamentals and Logical Foundations of Truth Maintenance, 1989, ISBN 917870-546-0.

No 239 Jonas Löwgren: Knowledge-Based Design Support and Discourse Management in User Interface Management Systems, 1991, ISBN 91-7870-720-X.

No 244 Henrik Eriksson: Meta-Tool Support for Knowledge Acquisition, 1991, ISBN 91-7870-746-3.

No 252 Peter Eklund: An Epistemic Approach to Interactive Design in Multiple Inheritance Hierarchies, 1991, ISBN 91-7870-784-6.

No 258 Patrick Doherty: NML3 - A Non-Monotonic Formalism with Explicit Defaults, 1991, ISBN 917870-816-8.

No 260 Nahid Shahmehri: Generalized Algorithmic Debugging, 1991, ISBN 91-7870-828-1.

No 264 Nils Dahlbäck: Representation of Discourse-Cognitive and Computational Aspects, 1992, ISBN 91-7870-8508.

No 265 Ulf Nilsson: Abstract Interpretations and Abstract Machines: Contributions to a Methodology for the Implementation of Logic Programs, 1992, ISBN 917870-858-3.

No 270 Ralph Rönnquist: Theory and Practice of Tensebound Object References, 1992, ISBN 91-7870-873-7.

No 273 Björn Fjellborg: Pipeline Extraction for VLSI Data Path Synthesis, 1992, ISBN 91-7870-880-X.

No 276 Staffan Bonnier: A Formal Basis for Horn Clause Logic with External Polymorphic Functions, 1992, ISBN 91-7870-896-6.

No 277 Kristian Sandahl: Developing Knowledge Management Systems with an Active Expert Methodology, 1992, ISBN 91-7870-897-4.

No 281 Christer Bäckström: Computational Complexity of Reasoning about Plans, 1992, ISBN 91-7870-979-2.

No 292 Mats Wirén: Studies in Incremental Natural Language Analysis, 1992, ISBN 91-7871-027-8.

No 297 Mariam Kamkar: Interprocedural Dynamic Slicing with Applications to Debugging and Testing, 1993, ISBN 91-7871-065-0.

No 302 Tingting Zhang: A Study in Diagnosis Using Classification and Defaults, 1993, ISBN 91-7871-078-2

No 312 Arne Jönsson: Dialogue Management for Natural Language Interfaces - An Empirical Approach, 1993, ISBN 91-7871-110-X.

No 338 Simin Nadjm-Tehrani: Reactive Systems in Physical Environments: Compositional Modelling and Framework for Verification, 1994, ISBN 91-7871-237-8.

No 371 Bengt Savén: Business Models for Decision Support and Learning. A Study of Discrete-Event Manufacturing Simulation at Asea/ABB 1968-1993, 1995, ISBN 91-7871-494-X. 
No 375 Ulf Söderman: Conceptual Modelling of Mode Switching Physical Systems, 1995, ISBN 91-7871-5164.

No 383 Andreas Kågedal: Exploiting Groundness in Logic Programs, 1995, ISBN 91-7871-538-5.

No 396 George Fodor: Ontological Control, Description, Identification and Recovery from Problematic Control Situations, 1995, ISBN 91-7871-603-9.

No 413 Mikael Pettersson: Compiling Natural Semantics, 1995, ISBN 91-7871-641-1.

No 414 Xinli Gu: RT Level Testability Improvement by Testability Analysis and Transformations, 1996, ISBN 91-7871-654-3.

No 416 Hua Shu: Distributed Default Reasoning, 1996, ISBN 91-7871-665-9.

No 429 Jaime Villegas: Simulation Supported Industrial Training from an Organisational Learning Perspective Development and Evaluation of the SSIT Method, 1996, ISBN 91-7871-700-0.

No 431 Peter Jonsson: Studies in Action Planning: Algorithms and Complexity, 1996, ISBN 91-7871-704-3.

No 437 Johan Boye: Directional Types in Logic Programming, 1996, ISBN 91-7871-725-6.

No 439 Cecilia Sjöberg: Activities, Voices and Arenas: Participatory Design in Practice, 1996, ISBN 91-7871728-0.

No 448 Patrick Lambrix: Part-Whole Reasoning in Description Logics, 1996, ISBN 91-7871-820-1.

No 452 Kjell Orsborn: On Extensible and Object-Relational Database Technology for Finite Element Analysis Applications, 1996, ISBN 91-7871-827-9.

No 459 Olof Johansson: Development Environments for Complex Product Models, 1996, ISBN 91-7871-855-4.

No 461 Lena Strömbäck: User-Defined Constructions in Unification-Based Formalisms, 1997, ISBN 91-7871857-0.

No 462 Lars Degerstedt: Tabulation-based Logic Programming: A Multi-Level View of Query Answering, 1996, ISBN 91-7871-858-9.

No 475 Fredrik Nilsson: Strategi och ekonomisk styrning - En studie av hur ekonomiska styrsystem utformas och används efter företagsförvärv, 1997, ISBN 91-7871914-3.

No 480 Mikael Lindvall: An Empirical Study of Requirements-Driven Impact Analysis in Object-Oriented Software Evolution, 1997, ISBN 91-7871-927-5.

No 485 Göran Forslund: Opinion-Based Systems: The Cooperative Perspective on Knowledge-Based Decision Support, 1997, ISBN 91-7871-938-0.

No 494 Martin Sköld: Active Database Management Systems for Monitoring and Control, 1997, ISBN 91-7219-0027.

No 495 Hans Olsén: Automatic Verification of Petri Nets in a CLP framework, 1997, ISBN 91-7219-011-6.

No 498 Thomas Drakengren: Algorithms and Complexity for Temporal and Spatial Formalisms, 1997, ISBN 917219-019-1.

No 502 Jakob Axelsson: Analysis and Synthesis of Heterogeneous Real-Time Systems, 1997, ISBN 91-7219-035-3.

No 503 Johan Ringström: Compiler Generation for DataParallel Programming Languages from Two-Level Semantics Specifications, 1997, ISBN 91-7219-045-0.

No 512 Anna Moberg: Närhet och distans - Studier av kommunikationsmönster i satellitkontor och flexibla kontor, 1997, ISBN 91-7219-119-8.
No 520 Mikael Ronström: Design and Modelling of a Parallel Data Server for Telecom Applications, 1998, ISBN 917219-169-4.

No 522 Niclas Ohlsson: Towards Effective Fault Prevention An Empirical Study in Software Engineering, 1998, ISBN 91-7219-176-7.

No 526 Joachim Karlsson: A Systematic Approach for Prioritizing Software Requirements, 1998, ISBN 917219-184-8.

No 530 Henrik Nilsson: Declarative Debugging for Lazy Functional Languages, 1998, ISBN 91-7219-197-x.

No 555 Jonas Hallberg: Timing Issues in High-Level Synthesis, 1998, ISBN 91-7219-369-7.

No 561 Ling Lin: Management of 1-D Sequence Data - From Discrete to Continuous, 1999, ISBN 91-7219-402-2.

No 563 Eva L Ragnemalm: Student Modelling based on Collaborative Dialogue with a Learning Companion, 1999, ISBN 91-7219-412-X.

No 567 Jörgen Lindström: Does Distance matter? On geographical dispersion in organisations, 1999, ISBN 917219-439-1.

No 582 Vanja Josifovski: Design, Implementation and Evaluation of a Distributed Mediator System for Data Integration, 1999, ISBN 91-7219-482-0.

No 589 Rita Kovordányi: Modeling and Simulating Inhibitory Mechanisms in Mental Image Reinterpretation Towards Cooperative Human-Computer Creativity, 1999, ISBN 91-7219-506-1.

No 592 Mikael Ericsson: Supporting the Use of Design Knowledge - An Assessment of Commenting Agents, 1999, ISBN 91-7219-532-0.

No 593 Lars Karlsson: Actions, Interactions and Narratives, 1999, ISBN 91-7219-534-7.

No 594 C. G. Mikael Johansson: Social and Organizational Aspects of Requirements Engineering Methods - A practice-oriented approach, 1999, ISBN 91-7219-541$\mathrm{X}$.

No 595 Jörgen Hansson: Value-Driven Multi-Class Overload Management in Real-Time Database Systems, 1999, ISBN 91-7219-542-8.

No 596 Niklas Hallberg: Incorporating User Values in the Design of Information Systems and Services in the Public Sector: A Methods Approach, 1999, ISBN 917219-543-6.

No 597 Vivian Vimarlund: An Economic Perspective on the Analysis of Impacts of Information Technology: From Case Studies in Health-Care towards General Models and Theories, 1999, ISBN 91-7219-544-4.

No 598 Johan Jenvald: Methods and Tools in ComputerSupported Taskforce Training, 1999, ISBN 91-7219. 547-9.

No 607 Magnus Merkel: Understanding and enhancing translation by parallel text processing, 1999, ISBN 917219-614-9.

No 611 Silvia Coradeschi: Anchoring symbols to sensory data, 1999, ISBN 91-7219-623-8.

No 613 Man Lin: Analysis and Synthesis of Reactive Systems: A Generic Layered Architecture Perspective, 1999, ISBN 91-7219-630-0.

No 618 Jimmy Tjäder: Systemimplementering i praktiken En studie av logiker i fyra projekt, 1999, ISBN 917219-657-2.

No 627 Vadim Engelson: Tools for Design, Interactive Simulation, and Visualization of Object-Oriented Models in Scientific Computing, 2000, ISBN 91-7219709-9. 
No 637 Esa Falkenroth: Database Technology for Control and Simulation, 2000, ISBN 91-7219-766-8.

No 639 Per-Arne Persson: Bringing Power and Knowledge Together: Information Systems Design for Autonomy and Control in Command Work, 2000, ISBN 91-7219796-X.

No 660 Erik Larsson: An Integrated System-Level Design for Testability Methodology, 2000, ISBN 91-7219-890-7.

No 688 Marcus Bjäreland: Model-based Execution Monitoring, 2001, ISBN 91-7373-016-5.

No 689 Joakim Gustafsson: Extending Temporal Action Logic, 2001, ISBN 91-7373-017-3.

No 720 Carl-Johan Petri: Organizational Information Provision - Managing Mandatory and Discretionary Use of Information Technology, 2001, ISBN-91-7373-126-9.

No 724 Paul Scerri: Designing Agents for Systems with Adjustable Autonomy, 2001, ISBN 9173732079.

No 725 Tim Heyer: Semantic Inspection of Software Artifacts: From Theory to Practice, 2001, ISBN 9173732087.

No 726 Pär Carlshamre: A Usability Perspective on Requirements Engineering - From Methodology to Product Development, 2001, ISBN 9173732125.

No 732 Juha Takkinen: From Information Management to Task Management in Electronic Mail, 2002, ISBN 91 73732583.

No 745 Johan Åberg: Live Help Systems: An Approach to Intelligent Help for Web Information Systems, 2002, ISBN 91-7373-311-3.

No 746 Rego Granlund: Monitoring Distributed Teamwork Training, 2002, ISBN 91-7373-312-1.

No 757 Henrik André-Jönsson: Indexing Strategies for Time Series Data, 2002, ISBN 917373-346-6.

No 747 Anneli Hagdahl: Development of IT-supported Interorganisational Collaboration - A Case Study in the Swedish Public Sector, 2002, ISBN 91-7373-314-8.

No 749 Sofie Pilemalm: Information Technology for NonProfit Organisations - Extended Participatory Design of an Information System for Trade Union Shop Stewards, 2002, ISBN 91-7373-318-0.

No 765 Stefan Holmlid: Adapting users: Towards a theory of use quality, 2002, ISBN 91-7373-397-0.

No 771 Magnus Morin: Multimedia Representations of Distributed Tactical Operations, 2002, ISBN 91-7373-4217.

No 772 Pawel Pietrzak: A Type-Based Framework for Locating Errors in Constraint Logic Programs, 2002, ISBN 91-7373-422-5.

No 758 Erik Berglund: Library Communication Among Programmers Worldwide, 2002, ISBN 91-7373-349-0.

No 774 Choong-ho Yi: Modelling Object-Oriented Dynamic Systems Using a Logic-Based Framework, 2002, ISBN 91-7373-424-1.

No 779 Mathias Broxvall: A Study in the Computational Complexity of Temporal Reasoning, 2002, ISBN 917373-440-3.

No 793 Asmus Pandikow: A Generic Principle for Enabling Interoperability of Structured and Object-Oriented Analysis and Design Tools, 2002, ISBN 91-7373-4799.

No 785 Lars Hult: Publika Informationstjänster. En studie av den Internetbaserade encyklopedins bruksegenskaper, 2003, ISBN 91-7373-461-6.

No 800 Lars Taxén: A Framework for the Coordination of Complex Systems' Development, 2003, ISBN 917373-604-X
No 808 Klas Gäre: Tre perspektiv på förväntningar och förändringar i samband med införande av informationssystem, 2003, ISBN 91-7373-618-X.

No 821 Mikael Kindborg: Concurrent Comics - programming of social agents by children, 2003, ISBN 91-7373-6511.

No 823 Christina Ölvingson: On Development of Information Systems with GIS Functionality in Public Health Informatics: A Requirements Engineering Approach, 2003, ISBN 91-7373-656-2.

No 828 Tobias Ritzau: Memory Efficient Hard Real-Time Garbage Collection, 2003, ISBN 91-7373-666-X.

No 833 Paul Pop: Analysis and Synthesis of CommunicationIntensive Heterogeneous Real-Time Systems, 2003, ISBN 91-7373-683-X.

No 852 Johan Moe: Observing the Dynamic Behaviour of Large Distributed Systems to Improve Development and Testing - An Empirical Study in Software Engineering, 2003, ISBN 91-7373-779-8.

No 867 Erik Herzog: An Approach to Systems Engineering Tool Data Representation and Exchange, 2004, ISBN 91-7373-929-4.

No 872 Aseel Berglund: Augmenting the Remote Control: Studies in Complex Information Navigation for Digital TV, 2004, ISBN 91-7373-940-5.

No 869 Jo Skåmedal: Telecommuting's Implications on Travel and Travel Patterns, 2004, ISBN 91-7373-9359.

No 870 Linda Askenäs: The Roles of IT - Studies of Organising when Implementing and Using Enterprise Systems, 2004, ISBN 91-7373-936-7.

No 874 Annika Flycht-Eriksson: Design and Use of Ontologies in Information-Providing Dialogue Systems, 2004, ISBN 91-7373-947-2.

No 873 Peter Bunus: Debugging Techniques for EquationBased Languages, 2004, ISBN 91-7373-941-3.

No 876 Jonas Mellin: Resource-Predictable and Efficient Monitoring of Events, 2004, ISBN 91-7373-956-1.

No 883 Magnus Bång: Computing at the Speed of Paper: Ubiquitous Computing Environments for Healthcare Professionals, 2004, ISBN 91-7373-971-5

No 882 Robert Eklund: Disfluency in Swedish human-human and human-machine travel booking dialogues, 2004, ISBN 91-7373-966-9.

No 887 Anders Lindström: English and other Foreign Linguistic Elements in Spoken Swedish. Studies of Productive Processes and their Modelling using FiniteState Tools, 2004, ISBN 91-7373-981-2.

No 889 Zhiping Wang: Capacity-Constrained Production-inventory systems - Modelling and Analysis in both a traditional and an e-business context, 2004, ISBN 9185295-08-6.

No 893 Pernilla Qvarfordt: Eyes on Multimodal Interaction, 2004, ISBN 91-85295-30-2.

No 910 Magnus Kald: In the Borderland between Strategy and Management Control - Theoretical Framework and Empirical Evidence, 2004, ISBN 91-85295-82-5.

No 918 Jonas Lundberg: Shaping Electronic News: Genre Perspectives on Interaction Design, 2004, ISBN 9185297-14-3.

No 900 Mattias Arvola: Shades of use: The dynamics of interaction design for sociable use, 2004, ISBN 9185295-42-6.

No 920 Luis Alejandro Cortés: Verification and Scheduling Techniques for Real-Time Embedded Systems, 2004, ISBN 91-85297-21-6. 
No 929 Diana Szentivanyi: Performance Studies of FaultTolerant Middleware, 2005, ISBN 91-85297-58-5.

No 933 Mikael Cäker: Management Accounting as Constructing and Opposing Customer Focus: Three Case Studies on Management Accounting and Customer Relations, 2005, ISBN 91-85297-64-X.

No 937 Jonas Kvarnström: TALplanner and Other Extensions to Temporal Action Logic, 2005, ISBN 91-85297-75-5.

No 938 Bourhane Kadmiry: Fuzzy Gain-Scheduled Visual Servoing for Unmanned Helicopter, 2005, ISBN 9185297-76-3

No 945 Gert Jervan: Hybrid Built-In Self-Test and Test Generation Techniques for Digital Systems, 2005, ISBN: 91-85297-97-6.

No 946 Anders Arpteg: Intelligent Semi-Structured Information Extraction, 2005, ISBN 91-85297-98-4.

No 947 Ola Angelsmark: Constructing Algorithms for Constraint Satisfaction and Related Problems - Methods and Applications, 2005, ISBN 91-85297-99-2.

No 963 Calin Curescu: Utility-based Optimisation of Resource Allocation for Wireless Networks, 2005, ISBN 91-85457-07-8.

No 972 Björn Johansson: Joint Control in Dynamic Situations, 2005, ISBN 91-85457-31-0.

No 974 Dan Lawesson: An Approach to Diagnosability Analysis for Interacting Finite State Systems, 2005, ISBN 91-85457-39-6.

No 979 Claudiu Duma: Security and Trust Mechanisms for Groups in Distributed Services, 2005, ISBN 91-8545754-X.

No 983 Sorin Manolache: Analysis and Optimisation of RealTime Systems with Stochastic Behaviour, 2005, ISBN 91-85457-60-4.

No 986 Yuxiao Zhao: Standards-Based Application Integration for Business-to-Business Communications, 2005, ISBN 91-85457-66-3.

No 1004 Patrik Haslum: Admissible Heuristics for Automated Planning, 2006, ISBN 91-85497-28-2.

No 1005 Aleksandra Tešanovic: Developing Reusable and Reconfigurable Real-Time Software using Aspects and Components, 2006, ISBN 91-85497-29-0.

No 1008 David Dinka: Role, Identity and Work: Extending the design and development agenda, 2006, ISBN 9185497-42-8.

No 1009 Iakov Nakhimovski: Contributions to the Modeling and Simulation of Mechanical Systems with Detailed Contact Analysis, 2006, ISBN 91-85497-43-X.

No 1013 Wilhelm Dahllöf: Exact Algorithms for Exact Satisfiability Problems, 2006, ISBN 91-85523-97-6.

No 1016 Levon Saldamli: PDEModelica - A High-Level Language for Modeling with Partial Differential Equations, 2006, ISBN 91-85523-84-4.

No 1017 Daniel Karlsson: Verification of Component-based Embedded System Designs, 2006, ISBN 91-85523-798

No 1018 Ioan Chisalita: Communication and Networking Techniques for Traffic Safety Systems, 2006, ISBN 9185523-77-1.

No 1019 Tarja Susi: The Puzzle of Social Activity - The Significance of Tools in Cognition and Cooperation, 2006, ISBN 91-85523-71-2.

No 1021 Andrzej Bednarski: Integrated Optimal Code Generation for Digital Signal Processors, 2006, ISBN 9185523-69-0.

No 1022 Peter Aronsson: Automatic Parallelization of Equation-Based Simulation Programs, 2006, ISBN 9185523-68-2.
No 1030 Robert Nilsson: A Mutation-based Framework for Automated Testing of Timeliness, 2006, ISBN 9185523-35-6.

No 1034 Jon Edvardsson: Techniques for Automatic Generation of Tests from Programs and Specifications, 2006, ISBN 91-85523-31-3.

No 1035 Vaida Jakoniene: Integration of Biological Data, 2006, ISBN 91-85523-28-3.

No 1045 Genevieve Gorrell: Generalized Hebbian Algorithms for Dimensionality Reduction in Natural Language Processing, 2006, ISBN 91-85643-88-2.

No 1051 Yu-Hsing Huang: Having a New Pair of Glasses Applying Systemic Accident Models on Road Safety, 2006, ISBN 91-85643-64-5.

No 1054 Åsa Hedenskog: Perceive those things which cannot be seen - A Cognitive Systems Engineering perspective on requirements management, 2006, ISBN 91-85643$57-2$.

No 1061 Cécile Åberg: An Evaluation Platform for Semantic Web Technology, 2007, ISBN 91-85643-31-9.

No 1073 Mats Grindal: Handling Combinatorial Explosion in Software Testing, 2007, ISBN 978-91-85715-74-9.

No 1075 Almut Herzog: Usable Security Policies for Runtime Environments, 2007, ISBN 978-91-85715-65-7.

No 1079 Magnus Wahlström: Algorithms, measures, and upper bounds for Satisfiability and related problems, 2007, ISBN 978-91-85715-55-8.

No 1083 Jesper Andersson: Dynamic Software Architectures, 2007, ISBN 978-91-85715-46-6.

No 1086 Ulf Johansson: Obtaining Accurate and Comprehensible Data Mining Models - An Evolutionary Approach, 2007, ISBN 978-91-85715-34-3.

No 1089 Traian Pop: Analysis and Optimisation of Distributed Embedded Systems with Heterogeneous Scheduling Policies, 2007, ISBN 978-91-85715-27-5.

No 1091 Gustav Nordh: Complexity Dichotomies for CSPrelated Problems, 2007, ISBN 978-91-85715-20-6.

No 1106 Per Ola Kristensson: Discrete and Continuous Shape Writing for Text Entry and Control, 2007, ISBN 97891-85831-77-7.

No 1110 He Tan: Aligning Biomedical Ontologies, 2007, ISBN 978-91-85831-56-2.

No 1112 Jessica Lindblom: Minding the body - Interacting socially through embodied action, 2007, ISBN 978-9185831-48-7.

No 1113 Pontus Wärnestål: Dialogue Behavior Management in Conversational Recommender Systems, 2007, ISBN 978-91-85831-47-0.

No 1120 Thomas Gustafsson: Management of Real-Time Data Consistency and Transient Overloads in Embedded Systems, 2007, ISBN 978-91-85831-33-3.

No 1127 Alexandru Andrei: Energy Efficient and Predictable Design of Real-time Embedded Systems, 2007, ISBN 978-91-85831-06-7.

No 1139 Per Wikberg: Eliciting Knowledge from Experts in Modeling of Complex Systems: Managing Variation and Interactions, 2007, ISBN 978-91-85895-66-3.

No 1143 Mehdi Amirijoo: QoS Control of Real-Time Data Services under Uncertain Workload, 2007, ISBN 97891-85895-49-6.

No 1150 Sanny Syberfeldt: Optimistic Replication with Forward Conflict Resolution in Distributed Real-Time Databases, 2007, ISBN 978-91-85895-27-4.

No 1155 Beatrice Alenljung: Envisioning a Future Decision Support System for Requirements Engineering - A Holistic and Human-centred Perspective, 2008, ISBN 978-91-85895-11-3. 
No 1156 Artur Wilk: Types for XML with Application to Xcerpt, 2008, ISBN 978-91-85895-08-3.

No 1183 Adrian Pop: Integrated Model-Driven Development Environments for Equation-Based Object-Oriented Languages, 2008, ISBN 978-91-7393-895-2.

No 1185 Jörgen Skågeby: Gifting Technologies - Ethnographic Studies of End-users and Social Media Sharing, 2008, ISBN 978-91-7393-892-1.

No 1187 Imad-Eldin Ali Abugessaisa: Analytical tools and information-sharing methods supporting road safety organizations, 2008, ISBN 978-91-7393-887-7.

No 1204 H. Joe Steinhauer: A Representation Scheme for Description and Reconstruction of Object Configurations Based on Qualitative Relations, 2008, ISBN 978-917393-823-5.

No 1222 Anders Larsson: Test Optimization for Core-based System-on-Chip, 2008, ISBN 978-91-7393-768-9.

No 1238 Andreas Borg: Processes and Models for Capacity Requirements in Telecommunication Systems, 2009, ISBN 978-91-7393-700-9.

No 1240 Fredrik Heintz: DyKnow: A Stream-Based Knowledge Processing Middleware Framework, 2009, ISBN 978-91-7393-696-5.

No 1241 Birgitta Lindström: Testability of Dynamic RealTime Systems, 2009, ISBN 978-91-7393-695-8.

No 1244 Eva Blomqvist: Semi-automatic Ontology Construction based on Patterns, 2009, ISBN 978-91-7393-6835.

No 1249 Rogier Woltjer: Functional Modeling of Constraint Management in Aviation Safety and Command and Control, 2009, ISBN 978-91-7393-659-0.

No 1260 Gianpaolo Conte: Vision-Based Localization and Guidance for Unmanned Aerial Vehicles, 2009, ISBN 978-91-7393-603-3.

No 1262 AnnMarie Ericsson: Enabling Tool Support for Formal Analysis of ECA Rules, 2009, ISBN 978-91-7393598-2.

No 1266 Jiri Trnka: Exploring Tactical Command and Control: A Role-Playing Simulation Approach, 2009, ISBN 978-91-7393-571-5.

No 1268 Bahlol Rahimi: Supporting Collaborative Work through ICT - How End-users Think of and Adopt Integrated Health Information Systems, 2009, ISBN 978-91-7393-550-0.

No 1274 Fredrik Kuivinen: Algorithms and Hardness Results for Some Valued CSPs, 2009, ISBN 978-91-7393-5258.

No 1281 Gunnar Mathiason: Virtual Full Replication for Scalable Distributed Real-Time Databases, 2009, ISBN 978-91-7393-503-6.

No 1290 Viacheslav Izosimov: Scheduling and Optimization of Fault-Tolerant Distributed Embedded Systems, 2009, ISBN 978-91-7393-482-4.

No 1294 Johan Thapper: Aspects of a Constraint Optimisation Problem, 2010, ISBN 978-91-7393-464-0.

No 1306 Susanna Nilsson: Augmentation in the Wild: User Centered Development and Evaluation of Augmented Reality Applications, 2010, ISBN 978-91-7393-416-9.

No 1313 Christer Thörn: On the Quality of Feature Models, 2010, ISBN 978-91-7393-394-0.

No 1321 Zhiyuan He: Temperature Aware and DefectProbability Driven Test Scheduling for System-onChip, 2010, ISBN 978-91-7393-378-0.

No 1333 David Broman: Meta-Languages and Semantics for Equation-Based Modeling and Simulation, 2010, ISBN 978-91-7393-335-3.
No 1337 Alexander Siemers: Contributions to Modelling and Visualisation of Multibody Systems Simulations with Detailed Contact Analysis, 2010, ISBN 978-91-7393317-9.

No 1354 Mikael Asplund: Disconnected Discoveries: Availability Studies in Partitioned Networks, 2010, ISBN 978-91-7393-278-3.

No 1359 Jana Rambusch: Mind Games Extended: Understanding Gameplay as Situated Activity, 2010, ISBN 978-91-7393-252-3.

No 1373 Sonia Sangari: Head Movement Correlates to Focus Assignment in Swedish,2011,ISBN 978-91-7393-1540 .

No 1374 Jan-Erik Källhammer: Using False Alarms when Developing Automotive Active Safety Systems, 2011, ISBN 978-91-7393-153-3.

No 1375 Mattias Eriksson: Integrated Code Generation, 2011, ISBN 978-91-7393-147-2.

No 1381 Ola Leifler: Affordances and Constraints of Intelligent Decision Support for Military Command and Control Three Case Studies of Support Systems, 2011, ISBN 978-91-7393-133-5.

No 1386 Soheil Samii: Quality-Driven Synthesis and Optimization of Embedded Control Systems, 2011, ISBN 978-91-7393-102-1.

No 1419 Erik Kuiper: Geographic Routing in Intermittentlyconnected Mobile Ad Hoc Networks: Algorithms and Performance Models, 2012, ISBN 978-91-7519-981-8.

No 1451 Sara Stymne: Text Harmonization Strategies for Phrase-Based Statistical Machine Translation, 2012, ISBN 978-91-7519-887-3.

No 1455 Alberto Montebelli: Modeling the Role of Energy Management in Embodied Cognition, 2012, ISBN 97891-7519-882-8.

No 1465 Mohammad Saifullah: Biologically-Based Interactive Neural Network Models for Visual Attention and Object Recognition, 2012, ISBN 978-91-7519-838-5.

No 1490 Tomas Bengtsson: Testing and Logic Optimization Techniques for Systems on Chip, 2012, ISBN 978-917519-742-5.

No 1481 David Byers: Improving Software Security by Preventing Known Vulnerabilities, 2012, ISBN 97891-7519-784-5.

No 1496 Tommy Färnqvist: Exploiting Structure in CSPrelated Problems, 2013, ISBN 978-91-7519-711-1.

No 1503 John Wilander: Contributions to Specification, Implementation, and Execution of Secure Software, 2013, ISBN 978-91-7519-681-7.

No 1506 Magnus Ingmarsson: Creating and Enabling the Useful Service Discovery Experience, 2013, ISBN 97891-7519-662-6.

No 1547 Wladimir Schamai: Model-Based Verification of Dynamic System Behavior against Requirements: Method, Language, and Tool, 2013, ISBN 978-917519-505-6.

No 1551 Henrik Svensson: Simulations, 2013, ISBN 978-917519-491-2.

No 1559 Sergiu Rafiliu: Stability of Adaptive Distributed RealTime Systems with Dynamic Resource Management, 2013, ISBN 978-91-7519-471-4.

No 1581 Usman Dastgeer: Performance-aware Component Composition for GPU-based Systems, 2014, ISBN 978-91-7519-383-0.

No 1602 Cai Li: Reinforcement Learning of Locomotion based on Central Pattern Generators, 2014, ISBN 978-917519-313-7. 
No 1652 Roland Samlaus: An Integrated Development Environment with Enhanced Domain-Specific Interactive Model Validation, 2015, ISBN 978-917519-090-7.

No 1663 Hannes Uppman: On Some Combinatorial Optimization Problems: Algorithms and Complexity, 2015, ISBN 978-91-7519-072-3.

No 1664 Martin Sjölund: Tools and Methods for Analysis, Debugging, and Performance Improvement of Equation-Based Models, 2015, ISBN 978-91-7519071-6.

No 1666 Kristian Stavåker: Contributions to Simulation of Modelica Models on Data-Parallel Multi-Core Architectures, 2015, ISBN 978-91-7519-068-6.

No 1680 Adrian Lifa: Hardware/Software Codesign of Embedded Systems with Reconfigurable and Heterogeneous Platforms, 2015, ISBN 978-91-7519040-2.

No 1685 Bogdan Tanasa: Timing Analysis of Distributed Embedded Systems with Stochastic Workload and Reliability Constraints, 2015, ISBN 978-91-7519-0228.

No 1691 Håkan Warnquist: Troubleshooting Trucks Automated Planning and Diagnosis, 2015, ISBN 97891-7685-993-3.

No 1702 Nima Aghaee: Thermal Issues in Testing of Advanced Systems on Chip, 2015, ISBN 978-91-7685-949-0.

No 1715 Maria Vasilevskaya: Security in Embedded Systems: A Model-Based Approach with Risk Metrics, 2015, ISBN 978-91-7685-917-9.

No 1729 Ke Jiang: Security-Driven Design of Real-Time Embedded System, 2015, ISBN 978-91-7685-884-4.

No 1733 Victor Lagerkvist: Strong Partial Clones and the Complexity of Constraint Satisfaction Problems: Limitations and Applications, 2015, ISBN 978-917685-856-1.

No 1734 Chandan Roy: An Informed System Development Approach to Tropical Cyclone Track and Intensity Forecasting, 2016, ISBN 978-91-7685-854-7.

Linköping Studies in Arts and Science

No 504 Ing-Marie Jonsson: Social and Emotional Characteristics of Speech-based In-Vehicle Information Systems: Impact on Attitude and Driving Behaviour, 2009, ISBN 978-91-7393-478-7.

No 586 Fabian Segelström: Stakeholder Engagement for Service Design: How service designers identify and communicate insights, 2013, ISBN 978-91-7519-554-4.

No 618 Johan Blomkvist: Representing Future Situations of Service: Prototyping in Service Design, 2014, ISBN 978-91-7519-343-4.

No 620 Marcus Mast: Human-Robot Interaction for SemiAutonomous Assistive Robots, 2014, ISBN 978-917519-319-9.

\section{Linköping Studies in Statistics}

No 9 Davood Shahsavani: Computer Experiments Designed to Explore and Approximate Complex Deterministic Models, 2008, ISBN 978-91-7393-976-8.

No 10 Karl Wahlin: Roadmap for Trend Detection and Assessment of Data Quality, 2008, ISBN 978-91-7393$792-4$.

No 11 Oleg Sysoev: Monotonic regression for large multivariate datasets, 2010, ISBN 978-91-7393-412-1.
No 13 Agné Burauskaite-Harju: Characterizing Temporal Change and Inter-Site Correlations in Daily and Subdaily Precipitation Extremes, 2011, ISBN 978-917393-110-6.

\section{Linköping Studies in Information Science}

No 1 Karin Axelsson: Metodisk systemstrukturering- att skapa samstämmighet mellan informationssystemarkitektur och verksamhet, 1998. ISBN-9172-19-296-8.

No 2 Stefan Cronholm: Metodverktyg och användbarhet en studie av datorstödd metodbaserad systemutveckling, 1998, ISBN-9172-19-299-2.

No 3 Anders Avdic: Användare och utvecklare - om anveckling med kalkylprogram, 1999. ISBN-91-7219606-8.

No 4 Owen Eriksson: Kommunikationskvalitet hos informationssystem och affärsprocesser, 2000, ISBN 917219-811-7.

No 5 Mikael Lind: Från system till process - kriterier för processbestämning vid verksamhetsanalys, 2001, ISBN 91-7373-067-X.

No 6 Ulf Melin: Koordination och informationssystem i företag och nätverk, 2002, ISBN 91-7373-278-8.

No 7 Pär J. Ågerfalk: Information Systems Actability - Understanding Information Technology as a Tool for Business Action and Communication, 2003, ISBN 917373-628-7.

No 8 Ulf Seigerroth: Att förstå och förändra systemutvecklingsverksamheter - en taxonomi för metautveckling, 2003, ISBN91-7373-736-4.

No 9 Karin Hedström: Spår av datoriseringens värden Effekter av IT i äldreomsorg, 2004, ISBN 91-7373963-4.

No 10 Ewa Braf: Knowledge Demanded for Action - Studies on Knowledge Mediation in Organisations, 2004, ISBN 91-85295-47-7.

No 11 Fredrik Karlsson: Method Configuration method and computerized tool support, 2005, ISBN 91-85297-48-8.

No 12 Malin Nordström: Styrbar systemförvaltning - Att organisera systemförvaltningsverksamhet med hjälp av effektiva förvaltningsobjekt, 2005, ISBN 91-85297-607.

No 13 Stefan Holgersson: Yrke: POLIS - Yrkeskunskap, motivation, IT-system och andra förutsättningar för polisarbete, 2005, ISBN 91-85299-43-X.

No 14 Benneth Christiansson, Marie-Therese Christiansson: Mötet mellan process och komponent mot ett ramverk för en verksamhetsnära kravspecifikation vid anskaffning av komponentbaserade informationssystem, 2006, ISBN 91-85643$22-\mathrm{X}$. 